\title{
DESIGN, FABRICATION, MODELING AND CHARACTERIZATION OF ELECTROSTATICALLY-ACTUATED SILICON MEMBRANES
}

\author{
A Thesis \\ Presented to \\ The Faculty of California Polytechnic State University, \\ San Luis Obispo
}

In Partial Fulfillment

Of the Requirements for the Degree

Master of Science in Engineering with Specialization in Materials Engineering

By

Brian C. Stahl

May 2009 
(C) 2009

Brian C. Stahl

ALL RIGHTS RESERVED 


\section{COMMITTEE MEMBERSHIP}

Title:

DESIGN, FABRICATION, MODELING, AND CHARACTERIZATION OF ELECTROSTATICALLYACTUATED SILICON MEMBRANES

Author: Brian C. Stahl

Date Submitted: $\quad$ May 2009

Committee Chair: Dr. Richard N. Savage Associate Professor of Materials Engineering California Polytechnic State University, San Luis Obispo

Committee Member: Dr. William L. Hughes Assistant Professor of Materials Science and Engineering Boise State University

Committee Member: Dr. Garret J. Hall

Associate Professor of Civil and Environmental Engineering California Polytechnic State University, San Luis Obispo 


\title{
ABSTRACT \\ Design, Fabrication, Modeling and Characterization of Electrostatically-Actuated Silicon Membranes
}

\author{
Brian C. Stahl
}

This thesis covers the design, fabrication, modeling and characterization of electrostatically actuated silicon membranes, with applications to microelectromechanical systems (MEMS). A microfabrication process was designed to realize thin membranes etched into a silicon wafer using a wet anisotropic etching process. These flexible membranes were bonded to a rigid counterelectrode using a photo-patterned gap layer. The membranes were actuated electrostatically by applying a voltage bias across the electrode gap formed by the membrane and the counterelectrode, causing the membrane to deflect towards the counterelectrode. This deflection was characterized for a range of actuating voltages and these results were compared to the deflections predicted by calculations and Finite Element Analysis (FEA). This thesis demonstrates the first electrostatically actuated MEMS device fabricated in the Cal Poly, San Luis Obispo Microfabrication Facility. Furthermore, this thesis should serve as groundwork for students who wish to improve upon the microfabrication processes presented herein, or who wish to fabricate thin silicon structures or electrostatically actuated MEMS structures of their own. 


\section{ACKNOWLEDGEMENTS}

First and foremost, the author thanks his committee and especially his advisors Prof. Richard N. Savage and Prof. William L. Hughes for their guidance and wisdom over the years, and for helping him realize just how much he has to learn. The author thanks the Cal Poly Microsystems Technology Group for their ideas, support, encouragement, assistance and discussion. The author is especially grateful to David Getchel for building some of the equipment used in this study, Dustin Dequine for helpful discussions about etching and Steven Meredith for his assistance with lithography mask design. The author is grateful to Hans Mayer for helpful discussions on the microfluidic applications of membranes. The author thanks Matthew Lewis, Dylan Chesbro, Sean Kaylor, Ryan Rivers, Daniel Marrujo, Eric Sackmann, Daniel Helms and Nicholas Vickers for their friendship and encouragement. The author thanks the entire faculty and staff of the Materials Engineering department for providing a superior undergraduate education, especially Prof. Linda Vanasupa for first introducing him to the department that would become his home for six and a half years. The author thanks his friends and family for their unending support and encouragement, through good times and bad. The author is especially grateful to Mr. Paul Bonderson for generously establishing the Bonderson Fellowship, from which the author has received financial support and the opportunity to pursue graduate studies. The author wishes to acknowledge support from the University of California, Santa Barbara Materials Research Laboratory and the Materials Research Facilities Network, especially Dr. Anika Odukale and Dr. Tom Mates. 


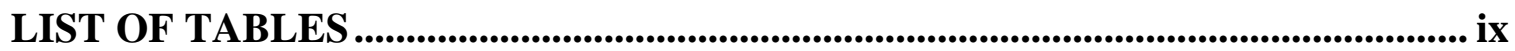

LIST OF FIGURES

LIST OF ABBREVIATIONS ............................................................................ii

LIST OF SYMBOLS

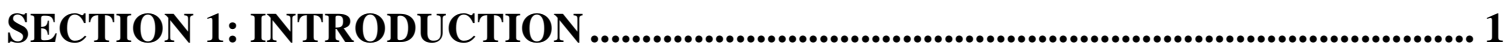

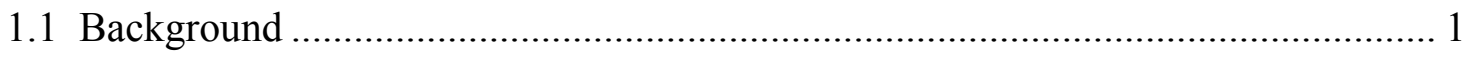

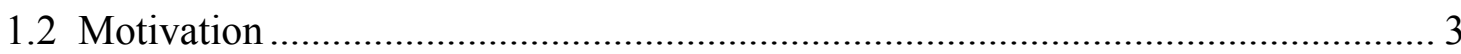

SECTION 2: DESIGN ............................................................................................................. 4

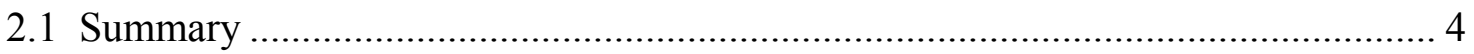

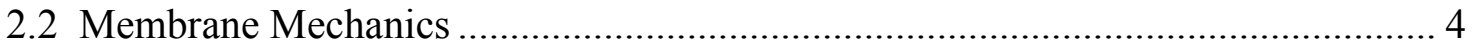

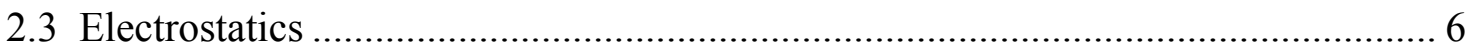

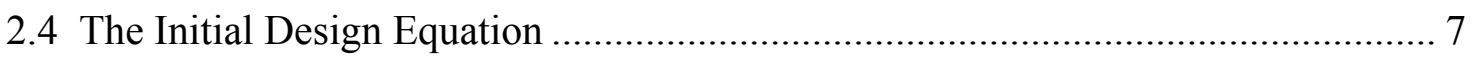

2.5 The Nonlinear Electrostatic Force Response ……….......................................... 8

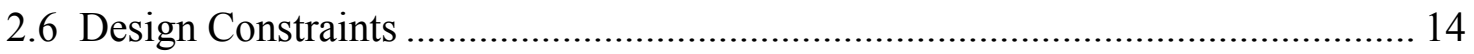

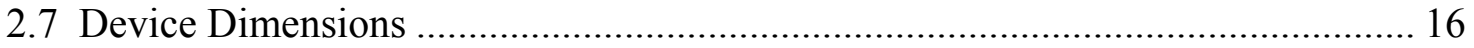

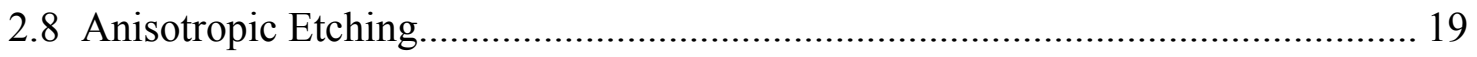

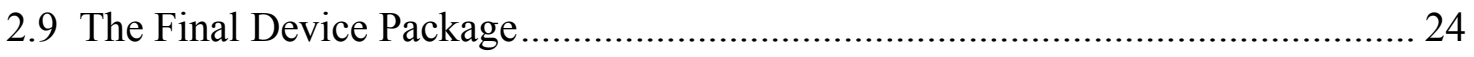




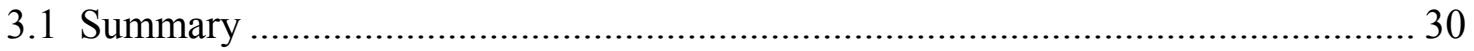

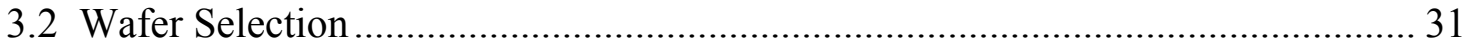

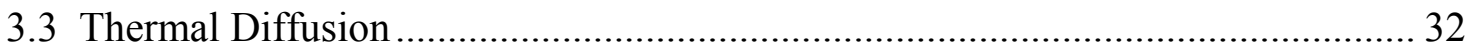

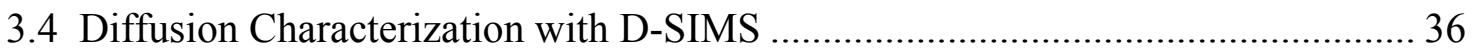

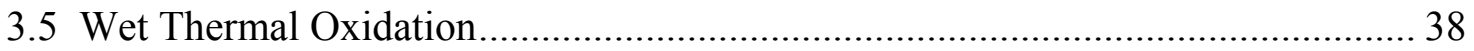

3.6 Positive Resist Photolithography and Patterning ................................................. 42

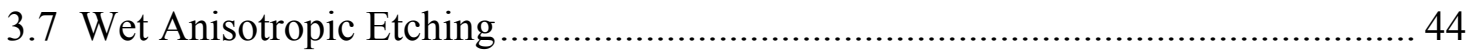

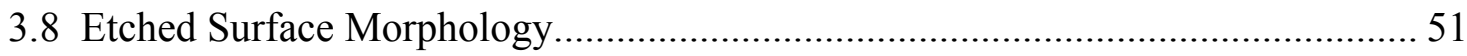

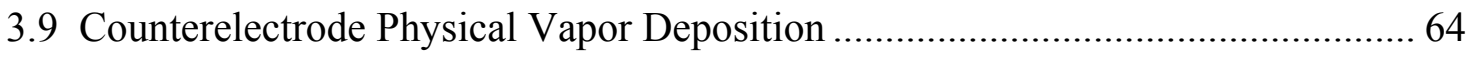

3.10 Counterelectrode Photolithography and Patterning ............................................. 65

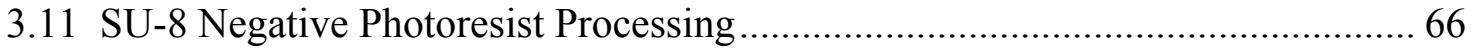

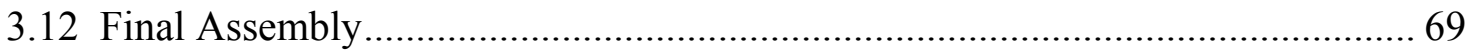

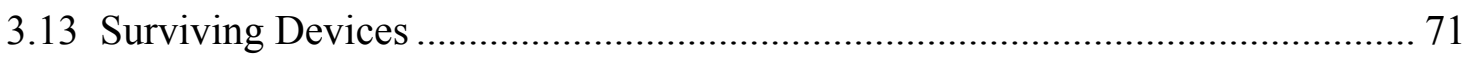

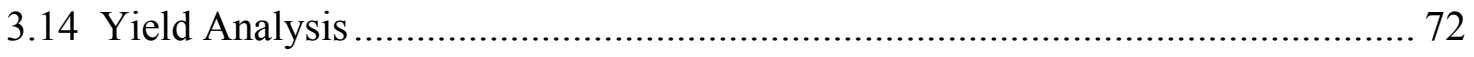

SECTION 4: MODELING .................................................................................................. 77

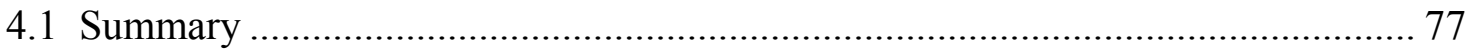

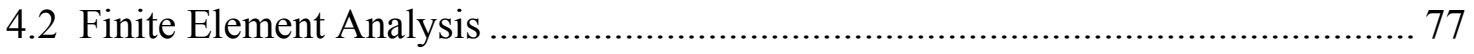

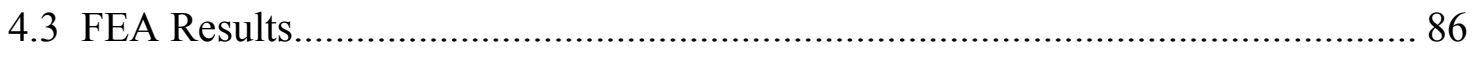

SECTION 5: DEVICE CHARACTERIZATION .......................................................... 90 
5.1 Summary

5.2 Actuation Setup 90

5.3 Interpreting the Results 93

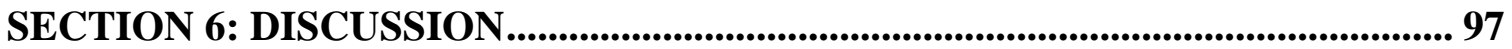

6.1 Modeling and Characterization Results ....................................................... 97

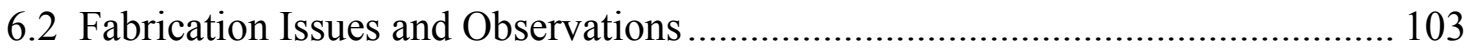

SECTION 7: CONCLUSIONS AND FUTURE WORK............................................ 108

REFERENCES

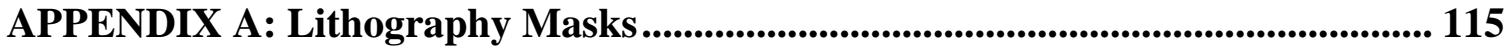

APPENDIX B: Raw and Normalized Deflection ................................................. 125

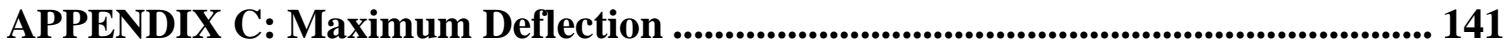

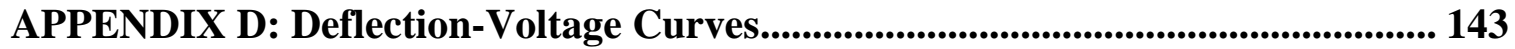

APPENDIX E: Initial Design Equation Model Comparison ....................................... 146 


\section{LIST OF TABLES}

Table 2.1: Membrane thicknesses and side lengths corresponding to a $22 \mu \mathrm{m}$ deflection.

Table 2.2: Etch window side lengths.

Table 3.1: Custom low-TTV wafer specifications for deep-etching applications.

Table 3.2: The two-step spin program used to coat wafers with spin-on dopant.

Table 3.3: Thermal diffusion process designed to heavily dope silicon wafers with boron to create an etch-stop layer.

Table 3.4: Thermal oxidation process designed to grow a 700nm wet oxide.

Table 3.5: 3-step spin process for positive photoresist application.

Table 3.6: Aluminum sputtering parameters.

Table 3.7: SU-8 coating spin-program designed to yield $\sim 40 \mu \mathrm{m}$ film.

Table 3.8: Soft-bake times and temperatures for $\sim 40 \mu$ m-thick SU-8.

Table 3.9: Post-exposure bake times and temperatures for $\sim 40 \mu \mathrm{m}$-thick SU-8.

Table 3.10: Surviving devices and their key dimensions.

Table 4.1: Generic CoventorWare process to model membranes.

Table 4.2: Base design dimensions for FEA.

Table 4.3: FEA investigation of device dimensions.

Table 6.1: Parameters used to model membrane deflection with hand calculations. 


\section{LIST OF FIGURES}

Figure 2.1: Coordinate system used with Eq. (2.2) and (2.3).

Figure 2.2: Behavior of ideal (left) and real (right) membranes under electrostatic actuation.

Figure 2.3: Force-displacement plot showing the idealized mechanical restoring force $\mathrm{kx}$ (straight line) and the electrostatic force for a series of actuation voltages (curves).

Figure 2.4: A standard (100) wafer, showing the orientation of the major flat and membrane.

Figure 2.5: An etch pit in (100) silicon showing the $54.74^{\circ}$ sidewalls.

Figure 2.6: Principal crystallographic planes for a cubic unit cell.

Figure 2.7: Schematic of the final device package.

Figure 2.8: A single die from the silicon etch window mask.

Figure 2.9: A counterelectrode for a $4,400 \mu \mathrm{m}$-wide membrane.

Figure 2.10: An SU-8 gap feature corresponding to a $4,400 \mu \mathrm{m}$ membrane.

Figure 2.11: A 3-D perspective view of an assembled device.

Figure 2.12: An exploded 3-D view of the final device.

Figure 2.13: The back side of the final device.

Figure 3.1: Orientation of the wafers in the boat prior to thermal diffusion.

Figure 3.2: Orientation of the tube furnace, wafer boat and gas flow (not to scale).

Figure 3.3: Boron concentration profiles after thermal diffusion.

Figure 3.4: Device wafers book-ended by dummy wafers, loaded in a quartz boat.

Figure 3.5: The reflux-condenser etch vessel.

Figure 3.6: A diagram of the reflux-condenser etch vessel.

Figure 3.7: Measuring the etch depth with a stylus profilometer. 
Figure 3.8: Crater-type defects observed on etched (100) surfaces.

Figure 3.9: Unusually large crater defects. $\quad 52$

Figure 3.10: A line of craters extending across an etch pit. 52

Figure 3.11: A profile scan representative of the shape of the crater defects. Horizontal axis units are microns, vertical axis units are nanometers.

Figure 3.12: Membranes exhibiting optical translucency. $\quad 58$

Figure 3.13: Membrane bowing after deep etching. 59

Figure 3.14: Membrane optical transmission as a function of membrane thickness. Legend contains approximate membrane thicknesses in microns. 63

Figure 3.15: Measuring membrane thickness with the stylus profilometer. 63

Figure 3.16: An etched aluminum counterelectrode on a Pyrex substrate. 66

Figure 3.17: The patterned SU-8 gap layer over the aluminum counterelectrode. 69

Figure 3.18: Alignment of the membrane to the counterelectrode and gap area. $\quad 70$

Figure 3.19: Back-side view of the incomplete die bonding. $\quad 70$

Figure 3.20: A bonded device ready to be separated and characterized. 71

Figure 3.22: Roughness and irregularity on a membrane due to a single bubble in the center of the membrane with a long residence time.

Figure 3.23: Rough membrane edges caused by insufficient etchant circulation from a physical barrier to circulation - in this case, a wafer cassette. $\quad 75$

Figure 4.1: The "stack" of layers used to create the Coventor model. 79

Figure 4.2: The FEA model containing only the counterelectrode and membrane. $\quad 80$

Figure 4.3: Typical output from Coventor FEA showing displacement. 82

Figure 4.4: Von Mises stress in a membrane under actuation. $\quad 83$

Figure 4.5: Charge distribution on the membrane under actuation. $\quad 84$

Figure 4.6: Mesh size convergence study. $\quad 86$ 
Figure 4.7: Results of the deflection vs. side length experiment.

Figure 4.8: Results of the deflection vs. electrode gap experiment. 88

Figure 4.9: Results of the deflection vs. membrane thickness experiment. 89

Figure 5.1: The actuation and characterization setup. $\quad 91$

Figure 5.2: The device with attached lead-wires. $\quad 92$

Figure 5.3: The DUT attached to the profilometer stage. 92

Figure 5.4: Superimposed profilometer scans of a typical membrane under actuation.

Figure 5.5: A representative normalized displacement plot. 95

Figure 5.6: A representative plot of maximum displacement vs. actuating voltage. 96

Figure 6.1: Comparison of modeling techniques to representative device data.

Figure 6.2: A comparison of the standard and scaled initial design equations with the measured deflection data for a representative device.

Figure 6.3: Fatigue test results.

Figure 6.4: Measured and predicted boron concentrations after a 5-hr thermal diffusion. 


\section{LIST OF ABBREVIATIONS}

\begin{tabular}{|c|c|}
\hline $3-\mathrm{D}$ & 3-Dimensional \\
\hline $\mathrm{AC}$ & Alternating Current \\
\hline $\mathrm{BOE}$ & Buffered Oxide Etchant \\
\hline D-SIMS & Dynamic Secondary Ion Mass Spectroscopy \\
\hline $\mathrm{DC}$ & Direct Current \\
\hline DI & De-Ionized \\
\hline DSP & Double-Sided Polished \\
\hline DUT & Device Under Testing \\
\hline EBSD & Electron Back-Scatter Diffraction \\
\hline FEA & Finite Element Analysis \\
\hline GUI & Graphical User Interface \\
\hline HDPE & High-Density Polyethylene \\
\hline $\mathrm{HF}$ & Hydrofluoric Acid \\
\hline $\mathrm{HV}$ & High Voltage \\
\hline IPA & Isopropyl Alcohol \\
\hline $\mathrm{KOH}$ & Potassium Hydroxide \\
\hline MEMS & Microelectromechanical Systems \\
\hline OCP & Open Circuit Potential \\
\hline PID & Proportional-Integral-Derivative \\
\hline RF & Radio Frequency \\
\hline RIE & Reactive Ion Etching \\
\hline RMS & Root Mean Squared \\
\hline
\end{tabular}




$\begin{array}{cl}\text { RPM } & \text { Rotations Per Minute } \\ \text { SSP } & \text { Single-Sided Polished } \\ \text { TEM } & \text { Transmission Electron Microscopy } \\ \text { TMAH } & \text { Tetramethyl Ammonium Hydroxide } \\ \text { TTV } & \text { Total Thickness Variation } \\ \text { UHP } & \text { Ultra-High Purity } \\ \text { XRD } & \text { X-Ray Diffraction }\end{array}$




\section{LIST OF SYMBOLS}

\begin{tabular}{|c|c|}
\hline $\mathrm{a}$ & Lattice Constant $[\AA]$ \\
\hline $\mathrm{a}$ & 1/2 Membrane Side Length [m] \\
\hline A & Parallel Plate Area $\left[\mathrm{m}^{2}\right]$ \\
\hline A & Oxidation Parameter $[\mu \mathrm{m}]$ \\
\hline $\mathrm{B}$ & Oxidation Parameter $\left[\mu \mathrm{m}^{2} / \mathrm{hr}\right]$ \\
\hline $\mathrm{b}$ & Beam Width $[\mathrm{m}]$ \\
\hline $\mathrm{C}$ & Capacitance $[\mathrm{F}]$ \\
\hline$C(x, t)$ & Dopant Concentration [atoms $/ \mathrm{cm}^{3}$ ] \\
\hline $\mathrm{C}_{\mathrm{s}}$ & Surface Dopant Concentration [atoms $/ \mathrm{cm}^{3}$ ] \\
\hline $\mathrm{D}$ & Plate Flexural Rigidity [Nm] \\
\hline $\mathrm{D}$ & Diffusion Coefficient $\left[\mathrm{cm}^{2} / \mathrm{sec}\right]$ \\
\hline $\mathrm{D}_{0}$ & Diffusivity $\left[\mathrm{cm}^{2} / \mathrm{sec}\right]$ \\
\hline $\mathrm{d}$ & Electrode Gap [m] \\
\hline $\mathrm{d}_{0}$ & Initial Electrode Gap [m] \\
\hline $\mathrm{E}$ & Young's Modulus [GPa] \\
\hline $\mathrm{E}_{\mathrm{A}}$ & Activation Energy [eV] \\
\hline erf & Error Function \\
\hline $\mathrm{F}$ & Force $[\mathrm{N}]$ \\
\hline $\mathrm{F}_{\text {res }}$ & Mechanical Restoring Force $[\mathrm{N}]$ \\
\hline $\mathrm{h}$ & Membrane thickness [m] \\
\hline I & Beam Bending Moment of Inertia $\left[\mathrm{m}^{4}\right]$ \\
\hline $\mathrm{k}$ & Boltzmann's Constant $\left[8.617^{*} 10^{-5} \mathrm{eV} / \mathrm{K}\right]$ \\
\hline
\end{tabular}




\begin{tabular}{|c|c|}
\hline $\mathrm{k}$ & Spring Constant $[\mathrm{N} / \mathrm{m}]$ \\
\hline $\mathrm{k}^{\prime}$ & Adjusted Spring Constant [N/m] \\
\hline $\mathrm{L}$ & Membrane Side Length [m] \\
\hline $\mathrm{M}(\mathrm{x})$ & Bending Moment $[\mathrm{Nm}]$ \\
\hline $\mathrm{p}$ & Pressure $[\mathrm{Pa}]$ \\
\hline $\mathrm{Q}_{0}$ & Dopant Dose $\left[\right.$ atoms $\left./ \mathrm{cm}^{2}\right]$ \\
\hline $\mathrm{T}$ & Temperature $[\mathrm{K}]$ \\
\hline $\mathrm{t}$ & Time $[\mathrm{sec}]$ \\
\hline $\mathrm{V}$ & Voltage [V] \\
\hline $\mathrm{V}(\mathrm{x})$ & Shear Force $[\mathrm{N}]$ \\
\hline $\mathrm{V}_{\mathrm{c}}$ & Critical Drive Voltage [V] \\
\hline $\mathrm{W}$ & Membrane Deflection [m] \\
\hline $\mathrm{w}^{\prime}(\mathrm{x})$ & Non-uniform Electrostatic Load [N/m] \\
\hline $\mathrm{w}_{0}$ & Initial Uniform Electrostatic Load [N/m] \\
\hline $\mathrm{W}_{\max }$ & Maximum Membrane Displacement [m] \\
\hline $\mathrm{x}$ & Membrane Displacement [m] \\
\hline $\mathrm{x}_{\mathrm{c}}$ & Critical Membrane Displacement [m] \\
\hline$X_{\mathrm{ox}}$ & Oxide Thickness [m] \\
\hline $\mathrm{z}$ & Etch Depth [m] \\
\hline$\varepsilon$ & Relative Permittivity [unitless] \\
\hline$\varepsilon_{0}$ & Permittivity of Free Space $\left[8.854 * 10^{-12} \mathrm{~F} / \mathrm{m}\right]$ \\
\hline$\theta(\mathrm{x})$ & Beam Slope $\left[\mathrm{Nm}^{2}\right]$ \\
\hline$v$ & Poisson's Ratio [unitless] \\
\hline
\end{tabular}


$\sigma_{\mathrm{XX}}$

$\tau$

$\Omega$
Normal Stress [MPa]

Existing Oxide Factor [hr]

Resistance [ohms] 


\section{SECTION 1: INTRODUCTION}

\subsection{Background}

Microelectromechanical systems (MEMS) have had a profound impact on society by enabling new technologies within a wide range of industries, such as automotive, aerospace, energy, defense, telecommunications, medicine and entertainment [1-2]. MEMS are typically composed of miniaturized transducer and actuator structures integrated with drive or signal processing electronics; this integration is advantageous to the manufacturing and packaging of MEMS devices, as it provides for a reduction in the size of the entire sensor/actuator package, more robust devices and lower cost [3]. MEMS devices can range in complexity from relatively simple membrane-based pressure sensors to complicated multi-axis inertial sensors or optical switches for telecommunications [3].

Regardless of complexity, most MEMS devices are actuated by one of a select few methods, including: electrostatic, piezoelectric, thermomechanical and shape-memory [3]. Electrostatic actuation is common in the MEMS industry as it can be implemented using the mechanical structures within the device, does not require a constant electric current like thermomechanical/shape-memory or electromagnetic actuation, offers response times commensurate with charge buildup on the electrodes, does not require exotic materials or heating and can provide sensing capabilities through capacitance measurements in addition to actuation [4]. All that is required for electrostatic actuation is a conducting or semiconducting actuating structure, a counterelectrode and a voltage 
source. The principle behind electrostatic actuation is the attractive force created by the separation of opposite charges; this shall be discussed in detail in the following sections. A few notable examples of devices employing this actuation scheme include digital micro-mirrors [5], grating light valves [6], micro-motors [7] and active probe tips for scanning probe microscopy [8].

As stated above, one of the key components of a MEMS device is the transducing or actuating mechanical structure. Common types of structures include torsional hinges, rotors and stators, fixed-fixed beams, cantilever beams, serpentine springs, plates and membranes. Membranes are of particular interest because of their broad applications as both sensing and actuating structures; notable examples include capacitive pressure sensors [9], force sensors [10], inkjet nozzles [11] and microfluidic pumps [12]. Fabrication of membrane structures poses certain challenges, however, as the membranes must be uniformly thick, have low surface roughness and be continuous and free from large defects which could create stress concentrations during actuation. Residual stresses must be considered when fabricating thin structures, as various thermal and doping processes create intrinsic stresses in structures which may or may not be desirable for a particular application. In addition, the membranes must be free from pinholes if the membrane is to be exposed to a fluid pressure differential. A simple and robust method to fabricate membranes integrated into a larger MEMS device is to etch the membrane into the same substrate as the rest of the device, using either a wet or reactive ion etching (RIE) process. Wet etching was selected for this research because the required 
equipment was available at the time, and because it is a relatively simple process compared to RIE.

\subsection{Motivation}

The motivation behind this thesis was to expand the knowledge and capabilities of the Cal Poly Microfabrication Facility by developing the processes and technology required to fabricate thin membranes in silicon, and to actuate these structures electrostatically. The technology developed over the course of this research can be applied to the fabrication of other, more complex thin structures in silicon, such as fixed-fixed beams and cantilevers, or other MEMS devices based on electrostatic actuation, such as torsional hinges or micro-mirror devices.

This thesis will discuss the design methodology followed which led to the final devices, the fabrication process which was developed to realize the devices, the modeling and Finite Element Analysis (FEA) which was performed to predict the performance of the devices and, finally, the characterization of the relationship between actuation voltage and displacement for each device. 


\section{SECTION 2: DESIGN}

\subsection{Summary}

This section will discuss the design process that resulted in the devices that were fabricated. This design process was started by utilizing a steady-state algebraic equation which predicts the displacement of a membrane based on membrane dimensions and actuation voltage. This equation was refined to take into consideration the increase in electrostatic force which occurs as the membrane deflects towards the counterelectrode. Once a suitable equation was determined, design constraints were imposed to determine the device dimensions. Photolithography masks were created to define the individual layers of the device. Finally, a 3-D rendering of the device is shown.

\subsection{Membrane Mechanics}

The design process was begun by considering the mechanics of square membranes separately from electrostatics. The ideal membrane can be approximated by a square plate of uniform thickness which is affixed to a rigid frame or constraint base along all four edges. Since the aspect ratio of these structures is quite high, i.e. on the order of 1000:1, under a distributed load the plate can be assumed to be in pure bending. The details of diaphragm mechanics can be found elsewhere [13], but a few equations relevant to the design process will be presented here. Equation (2.1) gives the flexural rigidity of the membrane, $\mathrm{D}$ :

$$
D=\frac{E h^{3}}{12\left(1-v^{2}\right)}
$$


where $E$ is Young's modulus, $h$ is the thickness of the membrane, and $v$ is Poisson's ratio. For a membrane under a uniform applied pressure loading $p$, the deflection $w$ is given by

$$
w(x, y)=0.0213 p \frac{a^{4}}{D}\left(1-\frac{x^{2}}{a^{2}}\right)^{2}\left(1-\frac{y^{2}}{a^{2}}\right)^{2}
$$

where $a$ is one-half the side length, and $x$ and $y$ are coordinates on the axes given in Figure $2.1[13]$.

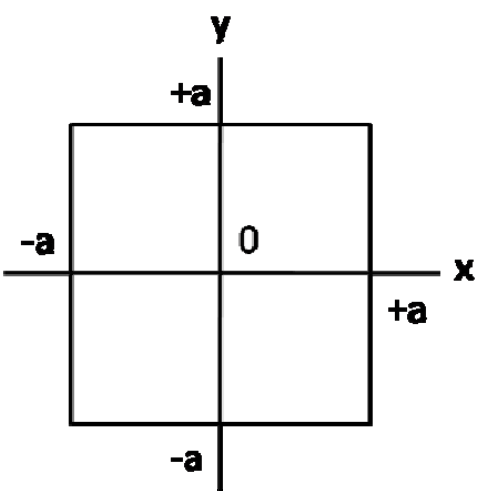

Figure 2.1: Coordinate system used with Eq. (2.2) and (2.3).

From Eq. (2.2), it is clear that the maximum displacement occurs in the center of the membrane. Eq. (2.3) [13] gives the normal stress $\sigma_{X X}$ in the membrane, and shows that the highest stresses in the membrane are found at the centers of each edge, i.e. at $( \pm a, 0)$ and $(0, \pm a)$.

$$
\sigma_{X X}=-0.51 p \frac{a^{2}}{h^{2}}\left[\left(1-\frac{x^{2}}{a^{2}}\right)^{2}\left(1-\frac{3 y^{2}}{a^{2}}\right)+v\left(1-\frac{y^{2}}{a^{2}}\right)^{2}\left(1-\frac{3 x^{2}}{a^{2}}\right)\right]
$$

Equations (2.2) and (2.3) are useful for calculating the general range of stress and deflection values expected for a membrane loaded by a uniform pressure. 


\subsection{Electrostatics}

Next, it is useful to examine the electrostatic force. As mentioned previously, the electrostatic force arises from the separation of electric charges or charge distributions, with opposite charges creating an attractive force and like charges creating a repulsive force. The membrane and counterelectrode can be treated as the two parallel plates of a capacitor, with a capacitance $C$ given by

$$
C=\frac{\varepsilon \varepsilon_{0} A}{d}
$$

where $A$ is the overlapping area of the parallel plates, $d$ is the gap between the plates, $\varepsilon_{0}$ is the permittivity of free space, or $\varepsilon_{0}=8.854 * 10^{-12} \mathrm{~F} / \mathrm{m}$, and $\varepsilon$ is the relative permittivity of the dielectric medium within the gap. When a voltage bias is applied across the plates the magnitude of the attractive normal force, $F$, acting on the plates as a result of the charge separation is given by

$$
F=\frac{C V^{2}}{2 d}
$$

where $V$ is the voltage bias applied across the plates.

Combining Eq. (2.4) and (2.5) results in a useful form of the equation for the normal force between two oppositely charged parallel plates:

$$
F=\frac{\varepsilon \varepsilon_{0} A V^{2}}{2 d^{2}}
$$

This above equation reasonably approximates the electrostatic force between the membrane and counterelectrode provided that the two are parallel and properly aligned. 


\subsection{The Initial Design Equation}

The rest of the design proceeded under the assumption that the electrostatic force acts as a uniformly applied pressure and that the plates do not deform; however, in reality the membrane does deform and the areas of the membrane which deform towards the counterelectrode experience a greater electrostatic force than those which do not deform. This assumption was necessary to proceed with the design process. In principle, a more sophisticated tool that does not make this assumption, such as software finite element analysis, could also be used to design the devices.

The initial goal of the design process was to design a membrane which, when actuated with a reasonable voltage, would deflect a distance that could be accurately measured with a stylus profilometer. A reasonable voltage was defined as one which could be generated using a standard laboratory DC power supply; $200 \mathrm{~V}$ was chosen as the design actuation voltage. The membrane was designed such that it should deflect on the order of tens of microns, which is approximately the deflection limit given the design constraints which will be described. Now, there are many factors which contribute to the deflection of a membrane, as Eq. (2.2) shows. If Eq. (2.1) and (2.2) are combined, $a$ is replaced with $L / 2$ (with $L$ being the side length), and only the center of the membrane is considered, the maximum deflection $w_{\max }$ is given by

$$
w_{\max }=0.015975 p \frac{\left(1-v^{2}\right) L^{4}}{E h^{3}}
$$

It is useful to further manipulate Eq. (2.7) by replacing $p$ with $F / L^{2}$, to find $w_{\max }$ as a function of the applied electrostatic force $F$ : 


$$
w_{\max }=0.015975 \frac{\left(1-v^{2}\right) F L^{2}}{E h^{3}}
$$

If $F$ is replaced with the equation for electrostatic force [Eq. (2.6)] and $L^{2}$ with the overlapping area $A$, the resulting equation relates the maximum deflection to the size and thickness of the membrane, the electrode gap, the material properties of the membrane and the actuating voltage.

$$
w_{\max }=0.015975 \frac{\left(1-v^{2}\right)}{E} \frac{\varepsilon \varepsilon_{0} A^{2} V^{2}}{2 d^{2} h^{3}}
$$

This equation uses the membrane area as the overlapping area of the parallel plates, which is a valid assumption provided that the membrane fully overlaps the counterelectrode. To ensure this, the counterelectrodes were designed to be approximately $1.5 \mathrm{~mm}$ longer and wider than the membrane to provide some tolerance for misalignment. Because the side length of the membrane and electrode is much larger than the spacing between them, fringe effects can be ignored [14] and there is no significant impact on the electrostatic force or deflection.

\subsection{The Nonlinear Electrostatic Force Response}

While equation (2.9) is useful for obtaining an order-of-magnitude estimate of the deflection of a membrane under electrostatic actuation, it does not account for the fact that the electrostatic force increases as the membrane deflects towards the counterelectrode because it was adapted from a static pressure loading equation. Instead, it treats the electrostatic force as a uniform, constant pressure loading denoted by $p$ in Eq. (2.7). From Eq. (2.6), it is clear that the electrostatic force increases proportionally to the 
square of the decrease in plate gap, i.e. if the gap is reduced by a factor of 2 , the force increases by a factor of 4 . In addition, the membrane exerts a mechanical restoring force when deflected which is analogous to the restoring force of a spring. Therefore, the design approach must consider the equilibrium between the mechanical restoring force and the electrostatic attractive force.

Let the membrane be treated as a simple Hooke's law spring, for which the mechanical restoring force $F_{\text {res }}$ is given by

$$
F_{\text {res }}=k x
$$

where $k$ is the effective spring constant of the membrane and $x$ is its displacement from equilibrium. This displacement is defined as

$$
x=d_{0}-d
$$

where $d_{0}$ is the initial electrode gap and $d$ is the current electrode gap. At equilibrium, this restoring force will be balanced by the electrostatic force $F$,

$$
F=F_{\text {res }}
$$

If $F, F_{\text {res }}$ and $d$ are substituted, the result is an equation for the equilibrium displacement of the membrane $x$, assuming a constant effective spring constant $k$ :

$$
k x=\frac{\varepsilon \varepsilon_{0} A V^{2}}{2\left(d_{0}-x\right)^{2}}
$$

This equation takes into account the increasing electrostatic force due to the displacement of the membrane towards the counterelectrode, however, it assumes that the entire membrane remains rigid and deflects uniformly towards the counterelectrode, experiencing some restoring force $k x$. This is illustrated in Figure 2.2. 

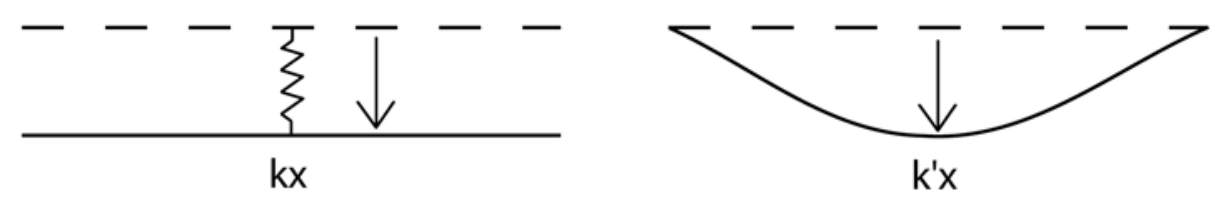

Figure 2.2: Behavior of ideal (left) and real (right) membranes under electrostatic actuation.

The validity of this assumption depends on the amount of deformation experienced by the membrane. For larger displacements, the membrane deforms more and becomes less plate-like, and hence the assumption becomes less valid. As Figure 2.2 shows, a real membrane remains fixed at the edges and deforms in the center towards the counterelectrode and experiences some complex restoring force $k^{\prime} x$. However, this approach still provides value because it considers the balance between the increasing electrostatic force and the mechanical restoring force.

To utilize Eq. (2.13) and determine the equilibrium displacement of the membrane, first an equation for the effective spring constant of the membrane should be developed. Eq. (2.8) relates the maximum displacement of the membrane to an applied load. If this equation is rearranged to solve for the applied load divided by the displacement, the result is an equation which can be used to estimate effective spring constant of the membrane, $k$ :

$$
k=\frac{F}{w_{\max }}=\frac{E h^{3}}{0.015975\left(1-v^{2}\right) L^{2}}
$$


This constant is similar to that of an idealized spring, and gives the relationship between the distributed load applied to the membrane and the deflection in the center of the membrane. Eq. (2.14) can replace $k$ in Eq. (2.13), resulting in

$$
\frac{E h^{3} x}{0.015975\left(1-v^{2}\right) L^{2}}=\frac{\varepsilon \varepsilon_{0} A V^{2}}{2\left(d_{0}-x\right)^{2}}
$$

which can be rearranged, and $L^{2}$ can be substituted for $A$ to solve for the equilibrium displacement $x$ :

$$
x\left(d_{0}-x\right)^{2}=\frac{0.015975 \varepsilon \varepsilon_{0}\left(1-v^{2}\right) V^{2} L^{4}}{2 E h^{3}}
$$

Thus, an equation emerges which can be solved for the equilibrium displacement of the square membrane as a function of material properties, device geometry and applied voltage.

Two consequences of the nonlinearity of the electrostatic force as the membrane deflects towards the counterelectrode are the concepts of pull-in and critical drive voltage. These subjects are treated in detail by Liu [14], but as they contributed to the design process it is useful to discuss the key points here. A plot of the mechanical restoring force $k x$ and the electrostatic force $F$ as a function of membrane displacement is shown in Figure 2.3. 


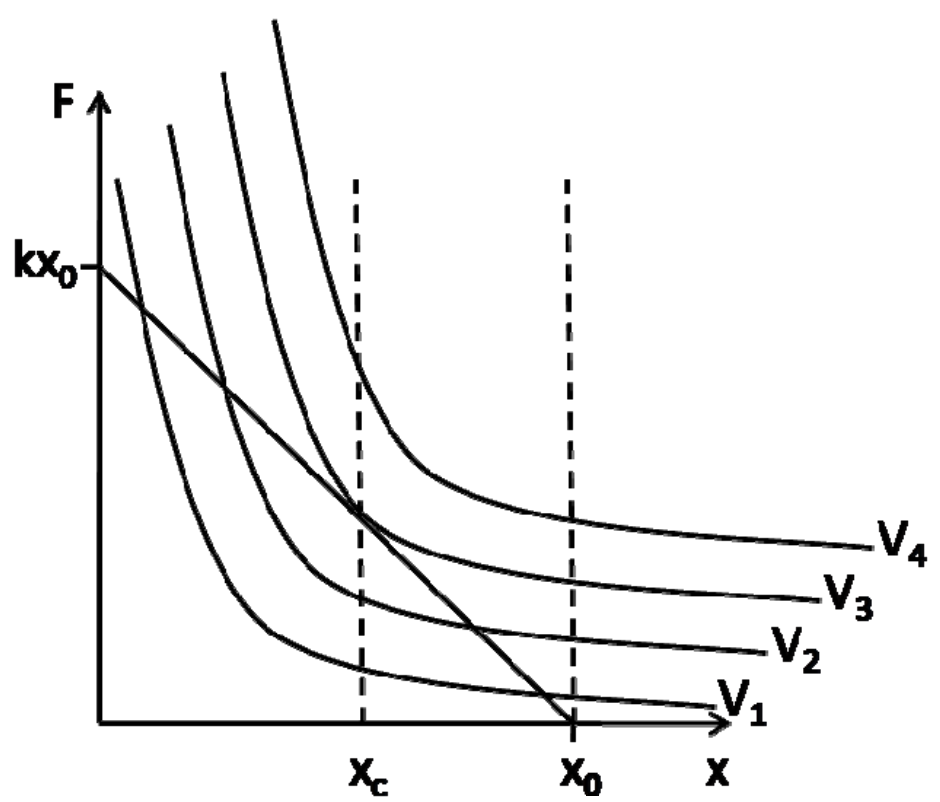

Figure 2.3: Force-displacement plot showing the idealized mechanical restoring force $\mathrm{kx}$ (straight line) and the electrostatic force for a series of actuation voltages (curves).

Here, $\mathrm{V}_{1}<\mathrm{V}_{2}<\mathrm{V}_{3}<\mathrm{V}_{4}$, and $\mathrm{x}_{\mathrm{c}}$ is the critical displacement of the membrane beyond which the electrostatic force overcomes the restoring force and the membrane experiences pull-in. From Figure 2.3 it is apparent that for each actuation voltage below some critical voltage, there exist two points where the restoring force equals the electrostatic force. One of these points is a stable equilibrium, i.e. if the membrane is deflected beyond the equilibrium point, it will return to equilibrium because the mechanical restoring force is greater than the electrostatic attractive force. The other intersection point is an unstable equilibrium, and any deviation from this point will result in either the mechanical restoring force returning the membrane to stable equilibrium or the electrostatic force drawing the membrane towards the counterelectrode, a condition called pull-in. If pull-in occurs, the membrane makes contact with the counterelectrode which can cause rupture if the stress in the membrane exceeds the tensile strength of the 
material. As the drive voltage increases, the stable equilibrium displacement increases until the two force curves are tangent.

The second concept, critical drive voltage $\left(V_{c}\right)$, is the voltage at which the electrostatic force curve is tangent to the restoring force curve, thus uniting the stable and unstable equilibrium points and creating a critical condition. This results in a critical equilibrium displacement $x_{c}$ beyond which pull-in will occur. This critical condition can be useful in the design process as it gives the maximum stable displacement of the membrane. Recognizing that the two force curves are tangent at this point, the corresponding displacement, i.e. the maximum stable displacement can be found. If the derivative of Eq. (2.13) is taken with respect to $x$ and this equation is substituted for $k$ in the equilibrium equation,

$$
\begin{gathered}
k=\frac{\varepsilon \varepsilon_{0} A V^{2}}{\left(d_{0}-x\right)^{3}} \\
\frac{\varepsilon \varepsilon_{0} A V^{2}}{2\left(d_{0}-x\right)^{2}}=x \frac{\varepsilon \varepsilon_{0} A V^{2}}{\left(d_{0}-x\right)^{3}}
\end{gathered}
$$

Eq. (2.18) is the result, which can be solved for $x$ to yield the critical displacement $x_{c}$. This critical displacement turns out to be

$$
x_{c}=\frac{d_{0}}{3}
$$

and is independent of device geometry, membrane stiffness, etc.

Finally, if $x_{c}$ is substituted for $x$ in Eq. (2.16), the resulting equation is:

$$
\frac{8}{27} d_{0}^{2}=\frac{0.015975 \varepsilon \varepsilon_{0}\left(1-v^{2}\right) V^{2} L^{4}}{E h^{3}}
$$


This equation was used to design the devices, as it gives the membrane side length and thickness required to produce maximum steady-state deflection for a given initial gap, drive voltage and material properties.

\subsection{Design Constraints}

At this point in the design process, design constraints were imposed to eliminate variables from the design equation until only the device geometry variables remained. The general goal was to design devices in a range of sizes and to optimize the design to maximize deflection, thus facilitating the characterization process. The first design constraint was to choose an actuation voltage, which was set at $200 \mathrm{~V}$ because of the general availability of laboratory power supplies capable of generating a $200 \mathrm{~V} \mathrm{DC}$ bias. The next design constraint was to set the electrode gap spacing at $67 \mu \mathrm{m}$. This was chosen for two reasons: first, a gap feature of this thickness could easily be fabricated using a patterned SU-8 negative photoresist layer; and second, a gap of this thickness would create an electric field inside the gap of roughly $3 * 10^{6} \mathrm{~V} / \mathrm{m}$, which is approximately the dielectric breakdown strength of air [15] and, thus, the maximum field that could be applied.

It should be noted that Paschen's law [16] predicts the breakdown voltage for parallel plates as a function of gas pressure, gas composition and gap distance between the plates. This breakdown potential $V_{\max }$ in volts is given by

$$
V_{\text {max }}=\frac{a(p d)}{\ln (p d)+b}
$$

where $a$ and $b$ are constants depending on the gas composition, $p$ is the pressure in atmospheres and $d$ is the plate gap in meters. When air at atmospheric pressure is the 
dielectric medium, $a=43.6 * 10^{6}$ and $b=12.8$. Paschen's law states that the electric field required to cause dielectric breakdown increases as the product $p d$ decreases, eventually reaching a maximum of $43 * 10^{6} \mathrm{~V} / \mathrm{m}$ at a gap distance of $7.5 \mu \mathrm{m}$. At a gap distance of 67 $\mu \mathrm{m}$, the breakdown potential given by Paschen's law is $916 \mathrm{~V}$, not $200 \mathrm{~V}$. Thus, the electrode gap could be decreased, the drive voltage could be increased, or both conditions could be applied without causing dielectric breakdown.

The next step was to select the appropriate material properties for the design, namely $E$ and $v$. The mechanical properties of single-crystal silicon are anisotropic, meaning that they vary depending on the orientation of the crystal structure. For example, in the (100) plane the Young's modulus for silicon varies from $130 \mathrm{GPa}$ in the $<100>$ direction to 168 GPa in the $<110>$ direction [17]. Likewise, Poisson's ratio varies significantly [18]. For a standard (100) silicon wafer, as shown in Figure 2.4, the face of the wafer is in the (100) plane and the $<110>$ direction is normal to the major flat. 


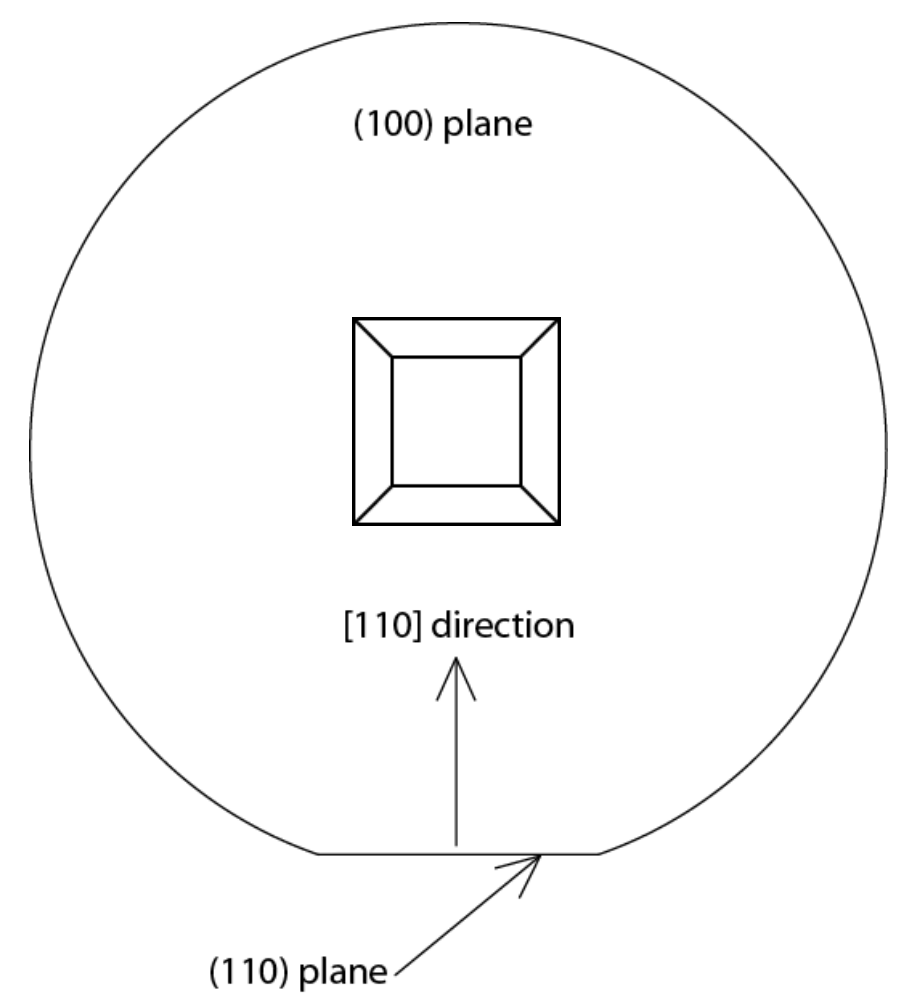

Figure 2.4: A standard (100) wafer, showing the orientation of the major flat and membrane.

Because of the anisotropic etching characteristics of single crystal silicon wafers, the membranes will be aligned with the major flat and their edges will be parallel or perpendicular to the $<110>$ direction as shown in Figure 2.4. The appropriate Young's modulus for the design calculations is $168 \mathrm{GPa}$ and 0.28 was used as Poisson's ratio in the $<100>$ direction [19].

\subsection{Device Dimensions}

The final step was to choose the device dimensions. This was approached by first determining what device thicknesses could reasonably be achieved, and then Eq. (2.20) was used to determine what membrane side length was required for critical deflection. A 
literature review revealed that heavily boron-doped silicon was commonly used as an etch stop to fabricate thin structures [20], and that a boron concentration of $\sim 5^{*} 10^{19}$ atoms $/ \mathrm{cm}^{3}$ should be sufficient for etch-stop performance [21]. To achieve a high boron concentration, a long pre-deposition thermal diffusion was performed using a spin-on boron source.

The equation governing pre-deposition diffusion is Fick's second law, given by

$$
\frac{\partial C(x, t)}{\partial t}=D \frac{\partial^{2} C(x, t)}{\partial x^{2}}
$$

where the concentration $C(x, t)$, in units of atoms $/ \mathrm{cm}^{3}$, is a function of the distance $x$ into the material and time $t$, and $D$ is the diffusion coefficient in $\mathrm{cm}^{2} / \mathrm{sec}$. The exact solution to Eq. (2.22) describing pre-deposition diffusion is reached by applying the initial condition that there is no dopant in the material before the diffusion, i.e.

$$
C(x, 0)=0
$$

and by applying two boundary conditions. The first boundary condition requires that the dopant concentration at the surface remains fixed at the dopant source concentration $C_{s}$ :

$$
C(0, t)=C_{s}
$$

This is known as the infinite source assumption. The second boundary condition requires that the dopant concentration is always zero at an infinite distance from the source:

$$
C(\infty, t)=0
$$

When these conditions are applied, an exact solution emerges that can be solved to find the depth for a specific dopant concentration at a given time:

$$
x=2 \sqrt{D t} * \operatorname{erfc}^{-1}\left(\frac{C(x, t)}{C_{s}}\right)
$$

Here, $\operatorname{erf} c^{-1}$ is the inverse complimentary error function, and $D$ is given by 


$$
D=D_{0} e^{\frac{-E_{A}}{k T}}
$$

where $D_{0}$ is the diffusivity factor depending on the dopant and the material being doped, $E_{A}$ is the activation energy of the reaction, $k$ is Boltzmann's constant and $T$ is the diffusion temperature in Kelvin. For the case of boron diffusing into silicon, $D_{0}=10.5$ $\mathrm{cm}^{2} / \mathrm{sec}$ and $E_{A}=3.69 \mathrm{eV}$; Boltzmann's constant $k=8.617^{*} 10^{-5} \mathrm{eV} / \mathrm{K}$, and the diffusion temperature was $1373 \mathrm{~K}$. Thus, the calculated diffusion coefficient $D$ was $2.99 * 10^{-13}$ $\mathrm{cm}^{2} / \mathrm{sec}$.

The diffusion time was set at 5 hours, which was the time limit of the diffusion furnace in the microfabrication facility. $C_{s}$, or the dopant source concentration, was effectively the solid solubility limit of boron in silicon which is approximately $2.4 * 10^{20}$ atoms $/ \mathrm{cm}^{3}$ at $1100^{\circ} \mathrm{C}[22]$. The reported boron concentration at which silicon starts to exhibit etchstop characteristics varies with etchant composition and temperature, but in general a concentration of approximately $5 * 10^{19}$ borons $/ \mathrm{cm}^{3}$ is sufficient to induce etch-stop characteristics [20-21, 23-26]. Eq. (2.26) was used to calculate an expected concentration of $5^{*} 10^{19}$ boron $/ \mathrm{cm}^{3}$ at a depth of $1.3 \mu \mathrm{m}$ after a 5 -hour diffusion at $1100^{\circ} \mathrm{C}$; however, due to a lack of first-hand experience with etch-stop behavior and an expectation that experimental results could differ from those presented in literature, it was prudent to design for the possibility that the membrane thickness could differ from the $1.3 \mu \mathrm{m}$ predicted value. It was thought unlikely that the membranes would be made much thinner than $1.3 \mu \mathrm{m}$ due to the increasing boron concentration closer to the surface of the wafer acting as a stronger etch-stop. However, it was thought possible that etch-stop characteristics could be observed at thicknesses greater than $1.3 \mu \mathrm{m}$ and so a range of 
devices were designed such that one of the devices would deflect enough to measure as long as the final membranes were $1-5 \mu \mathrm{m}$ thick.

Eq. (2.20) was used to design devices with 5 different side lengths, with each side length yielding the maximum displacement $\left(D_{0} / 3\right)$ for a given device thickness. Table 2.1 lists the expected membrane thicknesses and the corresponding side lengths $L$ which should yield maximum displacement for an actuation voltage of $200 \mathrm{~V}$ and an initial gap of 67 $\mu \mathrm{m}$. For convenience, the side lengths were rounded up to the nearest tenth of a millimeter when the lithography masks were drawn.

\begin{tabular}{|c|c|c|c|}
\hline $\begin{array}{c}\text { Device } \\
\text { Thickness }(\mu \mathrm{m})\end{array}$ & $\begin{array}{c}\text { Side } \\
\text { Length }(\mu \mathrm{m})\end{array}$ & $\begin{array}{c}\text { Rounded Side } \\
\text { Length }(\mu \mathrm{m})\end{array}$ & $\begin{array}{c}\text { Expected } \\
\text { Deflection }(\mu \mathrm{m})\end{array}$ \\
\hline 1 & 1300 & 1300 & 22 \\
\hline 2 & 2190 & 2200 & 22 \\
\hline 3 & 2970 & 3000 & 22 \\
\hline 4 & 3680 & 3700 & 22 \\
\hline 5 & 4350 & 4400 & 22 \\
\hline
\end{tabular}

Table 2.1: Membrane thicknesses and side lengths corresponding to a $22 \mu \mathrm{m}$ deflection.

\subsection{Anisotropic Etching}

Because silicon wafers are monocrystalline, certain chemical etchants may be used to anisotropically etch the substrate. These etchants yield predictable geometries, high aspect-ratio structures and smooth etched surfaces [25]. The two most common anisotropic or "direction-dependent" etching techniques for producing three-dimensional structures in silicon, such as those used in MEMS devices, are wet etching and dry etching; dry etching is also referred to as plasma or reactive ion etching (RIE). Wet etching is advantageous in that it is inexpensive, can be performed with commonly 
available chemicals such as potassium hydroxide $(\mathrm{KOH})$ or tetramethyl ammonium hydroxide (TMAH, $\left.\left(\mathrm{CH}_{3}\right)_{4} \mathrm{NOH}\right)$, and the resulting structures can be easily predicted from mask geometry and the crystallographic orientation of the substrate.

The generally accepted etching mechanism for silicon in alkaline solutions [27] proceeds as follows: first, surface silicon atoms react with hydroxyl groups, causing oxidation and 4 electrons to be injected into the conduction band of the silicon.

$$
\mathrm{Si}+2\left(\mathrm{OH}^{-}\right) \rightarrow \mathrm{Si}(\mathrm{OH})_{2}^{2+}+4 \mathrm{e}^{-}
$$

The 4 free electrons reduce water in the solution, evolving hydrogen gas and creating 4 new hydroxyl groups.

$$
4 \mathrm{e}^{-}+4 \mathrm{H}_{2} \mathrm{O} \rightarrow 4\left(\mathrm{OH}^{-}\right)+2 \mathrm{H}_{2}
$$

The silicon compound $\mathrm{Si}(\mathrm{OH})_{2}^{2+}$ reacts with 4 additional hydroxyl groups, resulting in the formation of water and a water-soluble silicon compound.

$$
\mathrm{Si}(\mathrm{OH})_{2}^{2+}+4\left(\mathrm{OH}^{-}\right) \rightarrow \mathrm{SiO}_{2}(\mathrm{OH})_{2}^{2-}+2 \mathrm{H}_{2} \mathrm{O}
$$

The overall reaction is:

$$
\mathrm{Si}+2\left(\mathrm{OH}^{-}\right)+2 \mathrm{H}_{2} \mathrm{O} \rightarrow \mathrm{SiO}_{2}(\mathrm{OH})_{2}^{2-}+2 \mathrm{H}_{2}
$$

In general, for anisotropic wet etching in silicon, etch rates increase with temperature because the 4 electrons involved in the reaction must be thermally excited into the silicon conduction band [27, 28-31]. Also, the etch rates along different crystallographic directions follow the general trend: $\operatorname{Rate}_{<111>}<\operatorname{Rate}_{<100>}<\operatorname{Rate}_{<110>}$. This anisotropy is due to a variation in the activation energy of the etching reaction with respect to the crystallographic plane [28]. Specifically, removing an atom from the (111) plane 
requires more energy than removing an atom from the (100) plane, and so on. Thus, anisotropic wet etchants tend to reveal the slanted (111) plane which is angled $54.74^{\circ}$ with respect to the (100) plane, as shown in Figure 2.5. Several theories exist which could explain why the (111) plane etches slower than the (110) and (100) planes [25]. One of the most prominent theories hypothesizes that the (111) plane etches slowest because surface atoms on this plane have only one "dangling" bond, and two are required by the above etching mechanism for the initial hydroxyl bonding. To remove an atom from this plane, three backbone bonds must be destabilized and broken, which requires that more binding electrons be thermally excited into the conduction band [28]. This is in contrast to surface atoms on the (100) plane which have only two backbone bonds and therefore have two "dangling" bonds. Heavily boron-doped silicon etches slower than undoped silicon due to the shrinking of the space-charge region at the surface of the silicon; this smaller space-charge region causes conduction band electrons generated by the oxidation reaction mentioned above to recombine with valence band holes, retarding the reduction of water and the entire etching process [24].

The $54.74^{\circ}$ angle between the (111) and (100) planes can be derived using the (110) plane, shown on the cubic unit cell with lattice constant $a$ in Figure 2.6. 


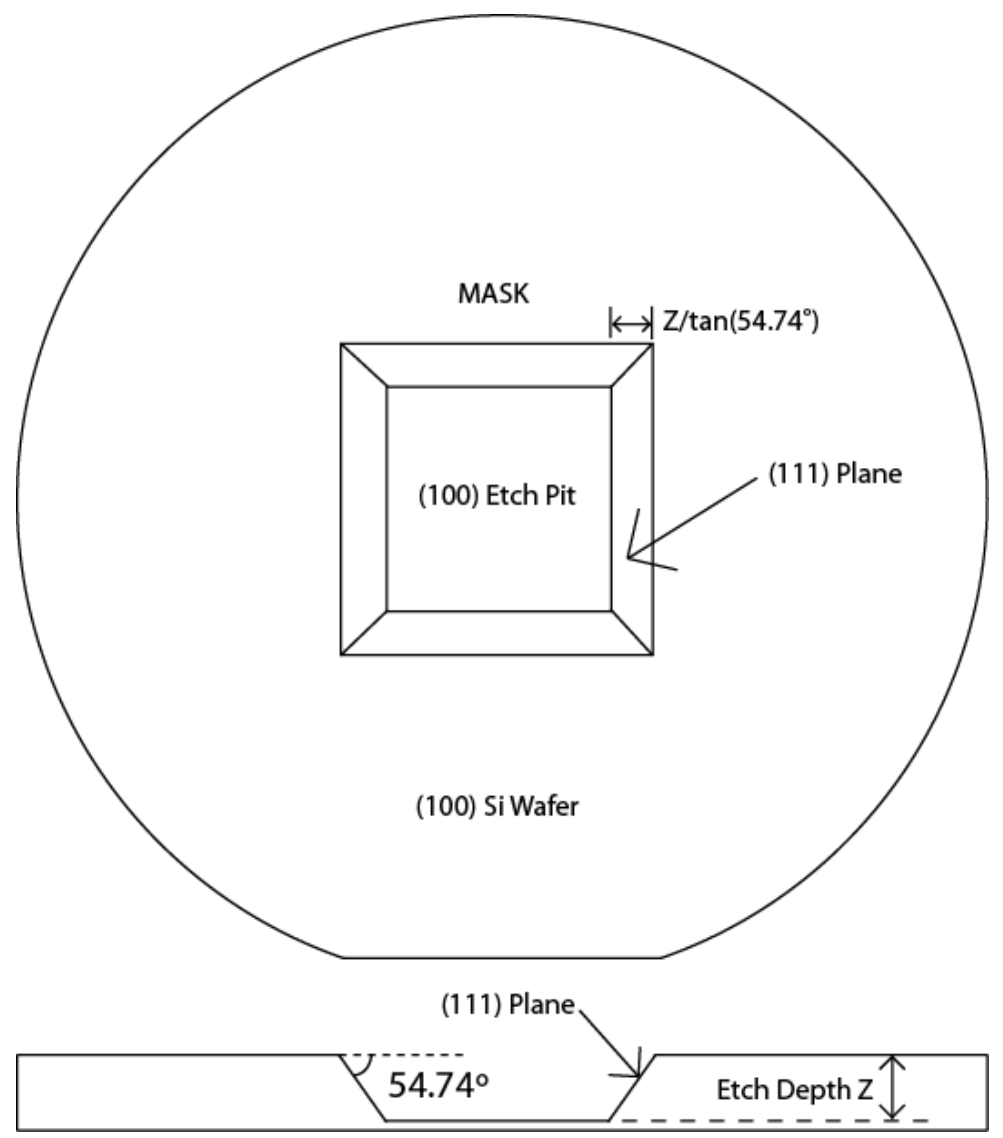

Figure 2.5: An etch pit in (100) silicon showing the $54.74^{\circ}$ sidewalls.

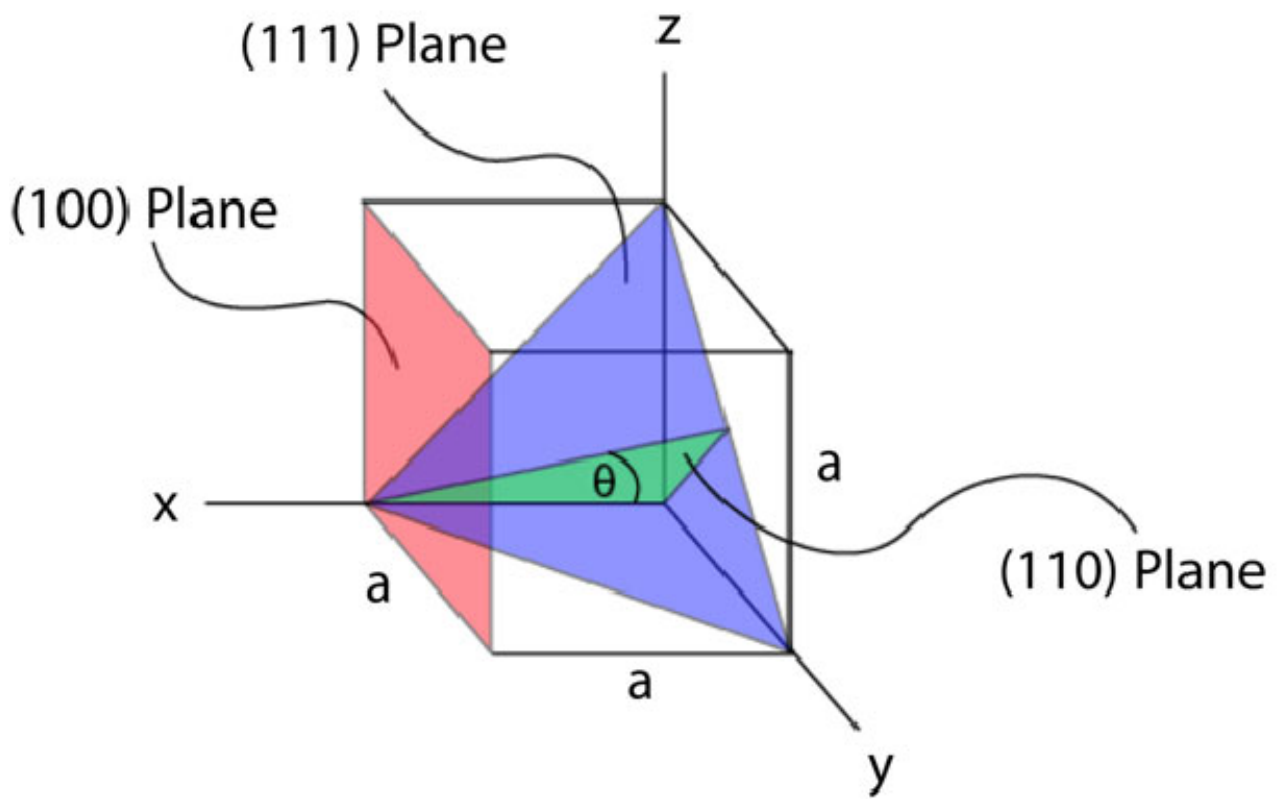

Figure 2.6: Principal crystallographic planes for a cubic unit cell. 
In a cubic unit cell with side length a, the angle between the (100) plane (red square) and the (111) plane (blue triangle) is the complementary angle of $\theta$, i.e. $90^{\circ}-\theta$. Here, $\theta$ is measured in the (110) plane (green triangle) and can be derived using trigonometry with the following equation:

$$
\theta=\tan ^{-1}\left(\frac{a \sqrt{2}}{2 a}\right)
$$

Here, the side of the triangle opposite the angle $\theta$ has a side length of $\frac{a \sqrt{2}}{2}$ and the side adjacent to $\theta$ has length $a$. Thus, $\theta=35.26^{\circ}$, and the complimentary angle is $54.74^{\circ}$.

This angle is significant because if anisotropic wet etching is used to fabricate device structures, the mask features must be larger than the final device features to account for the trapezoidal reduction in feature size. Specifically, mask features must be $Z / \tan \left(54.74^{\circ}\right)$ wider on each side, where $Z$ is the etch depth. Table 2.2 lists the final membrane side lengths, as well as the etch window dimensions required to obtain the desired side length after etching through approximately $400 \mu \mathrm{m}$ of silicon.

\begin{tabular}{|c|c|}
\hline Membrane Side Length $(\mu \mathrm{m})$ & Etch Window Side Length $(\mu \mathrm{m})$ \\
\hline 1300 & 1860 \\
\hline 2200 & 2760 \\
\hline 3000 & 3560 \\
\hline 3700 & 4260 \\
\hline 4400 & 4960 \\
\hline
\end{tabular}

Table 2.2: Etch window side lengths. 


\subsection{The Final Device Package}

Once the membranes themselves were designed, the issue of counterelectrode integration was considered. SU-8, an epoxy-based negative photoresist obtained from Microchem, was previously used as a thermally-cured adhesive to bond wafers together [32] and so a patterned SU-8 film was chosen to both define the electrode gap thickness and bond the silicon die containing the membrane to the die containing the counterelectrode. This is illustrated in Figure 2.7.

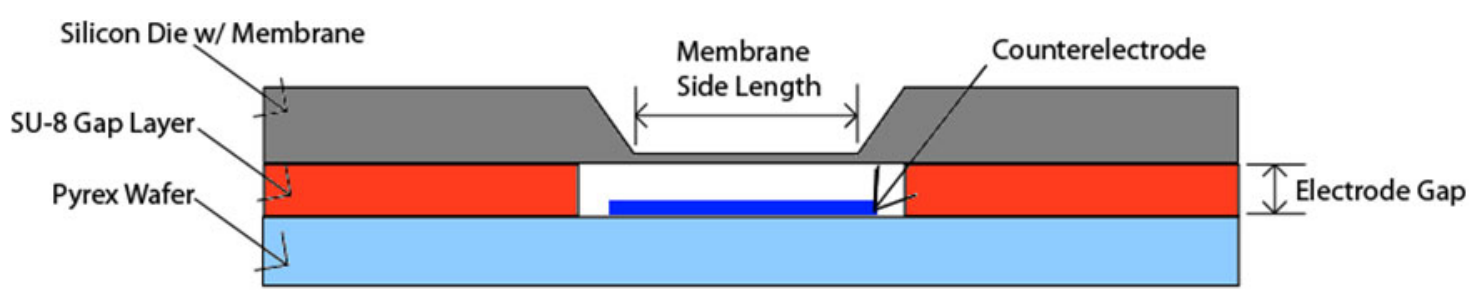

Figure 2.7: Schematic of the final device package.

The final step in the design process was to draw lithography masks for each layer of the device: the patterned counterelectrode, SU-8 gap and silicon etch windows for the membrane layer. AutoCAD 2007 was used to draw and dimension the masks, which are attached as Appendix A. Figures 2.8, 2.9 and 2.10 show a single die from each lithography mask. In addition to the etch windows on the mask for the silicon membrane layer, $50 \mu \mathrm{m}$ wide borders were drawn around each etch window to define the individual dies on the wafer and facilitate the wafer-dicing process (Figure 2.8). During the etching process the area within the border was etched and eventually a trench with a triangular cross-section defined by the intersection of two (111) planes was formed; this intersection acted as a stress concentration site when the wafer was bent, confining fracture to the 
trenches. The $4,960 \mu \mathrm{m}$ square etch window in Figure 2.8 created a membrane approximately $4,400 \mu \mathrm{m}$ wide when etched through $400 \mu \mathrm{m}$ of silicon. The $50 \mu \mathrm{m}$ border is shown surrounding the etch window, defining the individual silicon dies.

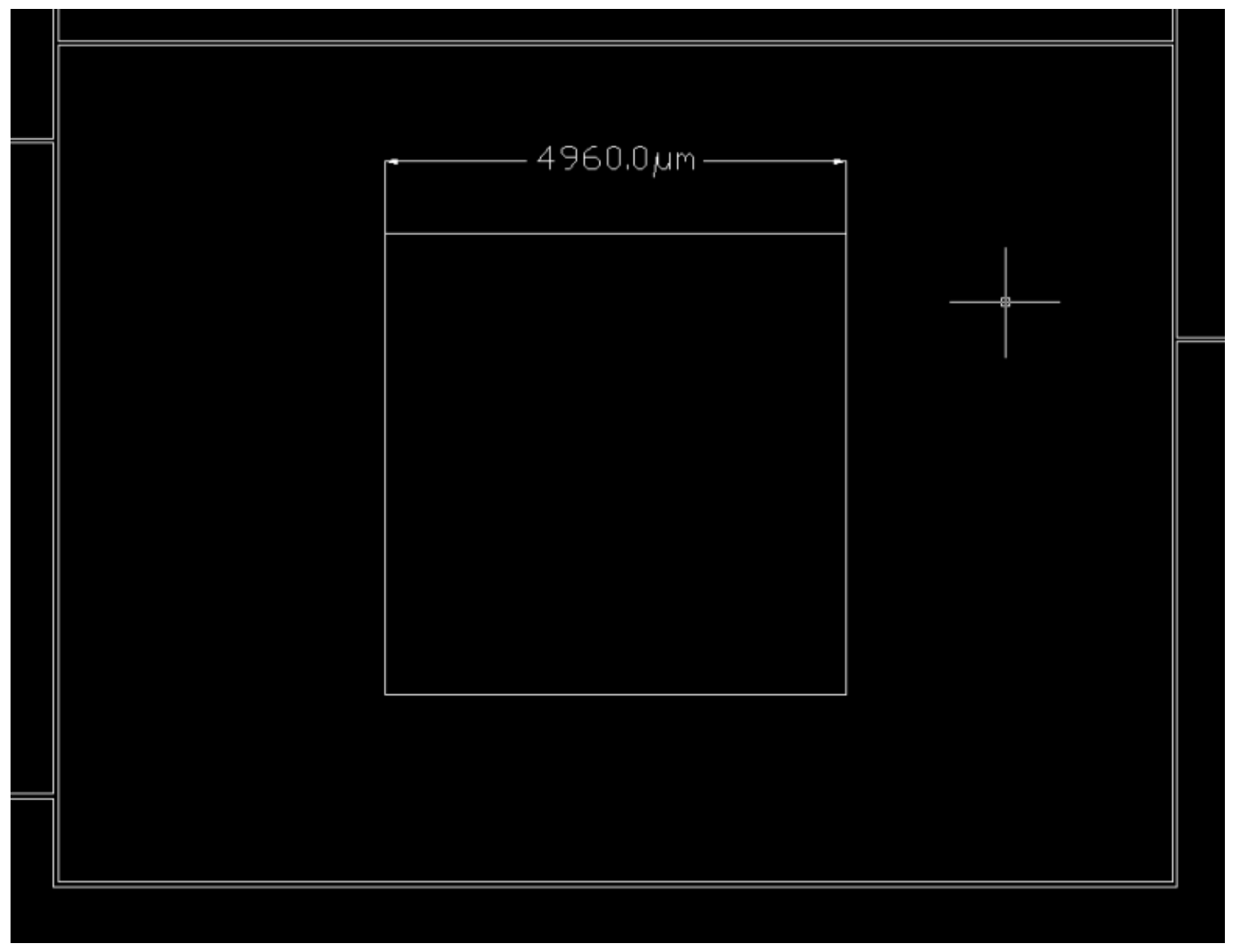

Figure 2.8: A single die from the silicon etch window mask.

The electrode mask consists of the square counterelectrode region, and a rectangular bond pad region for connecting a lead wire to the counterelectrode (Figure 2.9). During assembly, the membrane will be aligned and centered over the counterelectrode. The dimensions of this particular counterelectrode are shown in white. 


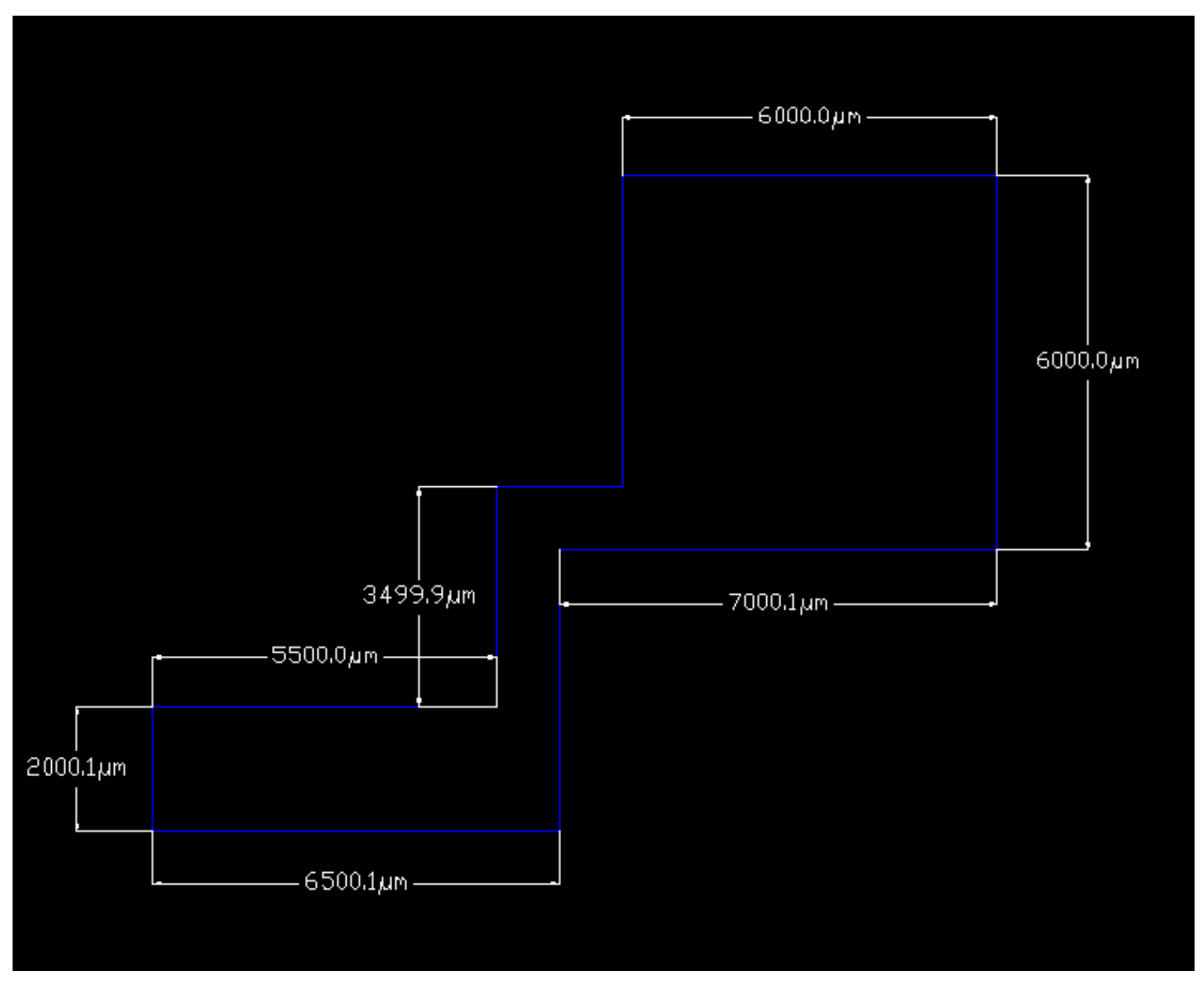

Figure 2.9: A counterelectrode for a $4,400 \mu \mathrm{m}$-wide membrane.

The SU-8 mask contains a square cavity which is aligned with the counterelectrode and the membrane (Figure 2.10). During actuation, the membrane deflects into this cavity towards the counterelectrode. The two channels on the top and right sides expose the cavity to the outside environment, preventing the cavity from sealing and pressurizing under actuation. In addition, the channels were intended to be viewing ports through which actuation could be observed and characterized. This SU-8 layer also includes alignment marks on both the sides and bottom of the cavity to facilitate alignment during 
assembly. Dimensions of this SU-8 gap feature, corresponding to a $4,400 \mu \mathrm{m}$ membrane, are shown in white.

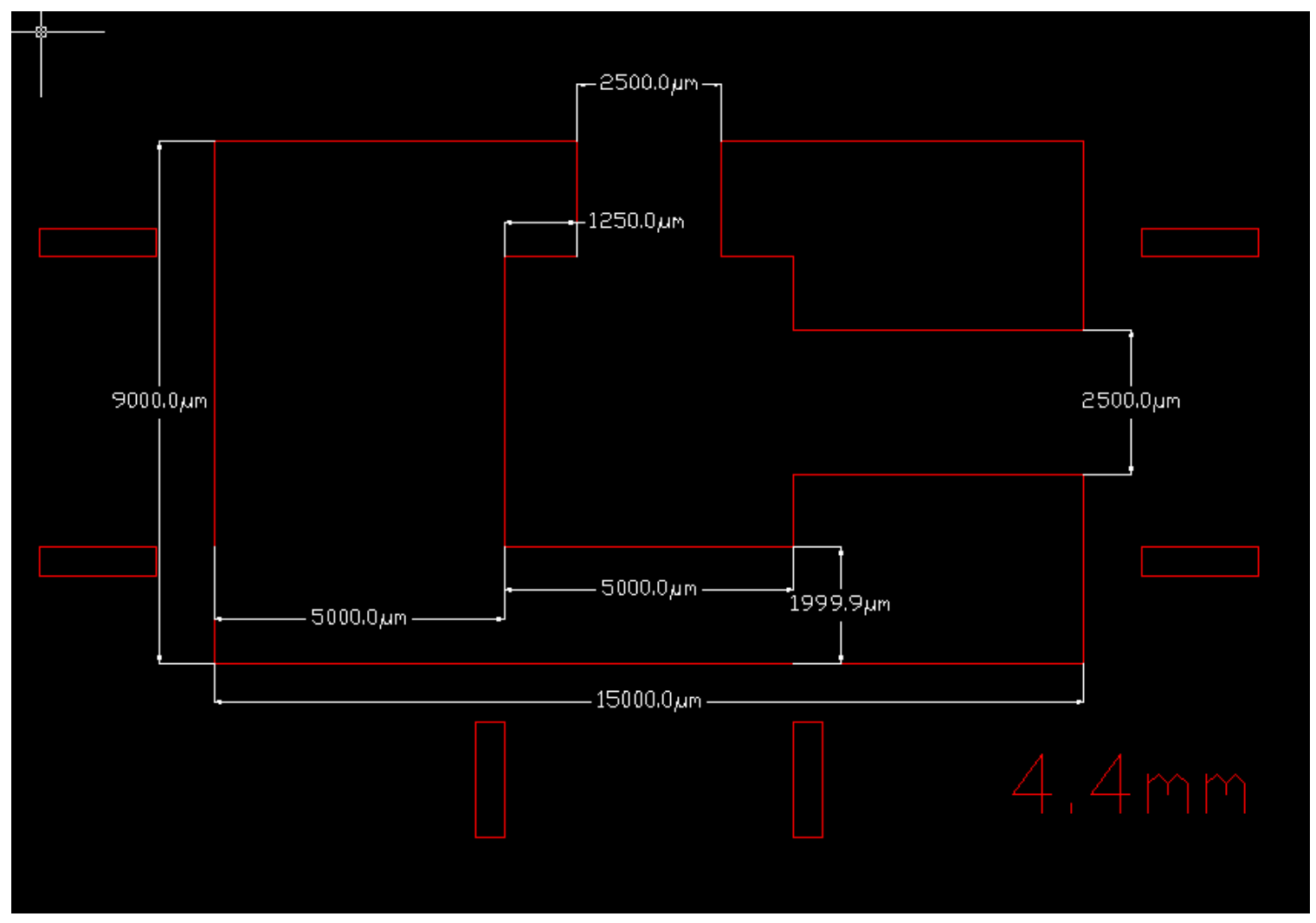

Figure 2.10: An SU-8 gap feature corresponding to a $4,400 \mu \mathrm{m}$ membrane.

A 3-D model of the device after assembly is shown in Figure 2.11. The three sets of alignment marks were used to position the silicon die so that the membrane is directly over the counterelectrode and SU-8 cavity. An exploded 3-D model of the device, as well as a view from the back of the device, can be seen in Figures 2.12 and 2.13. 


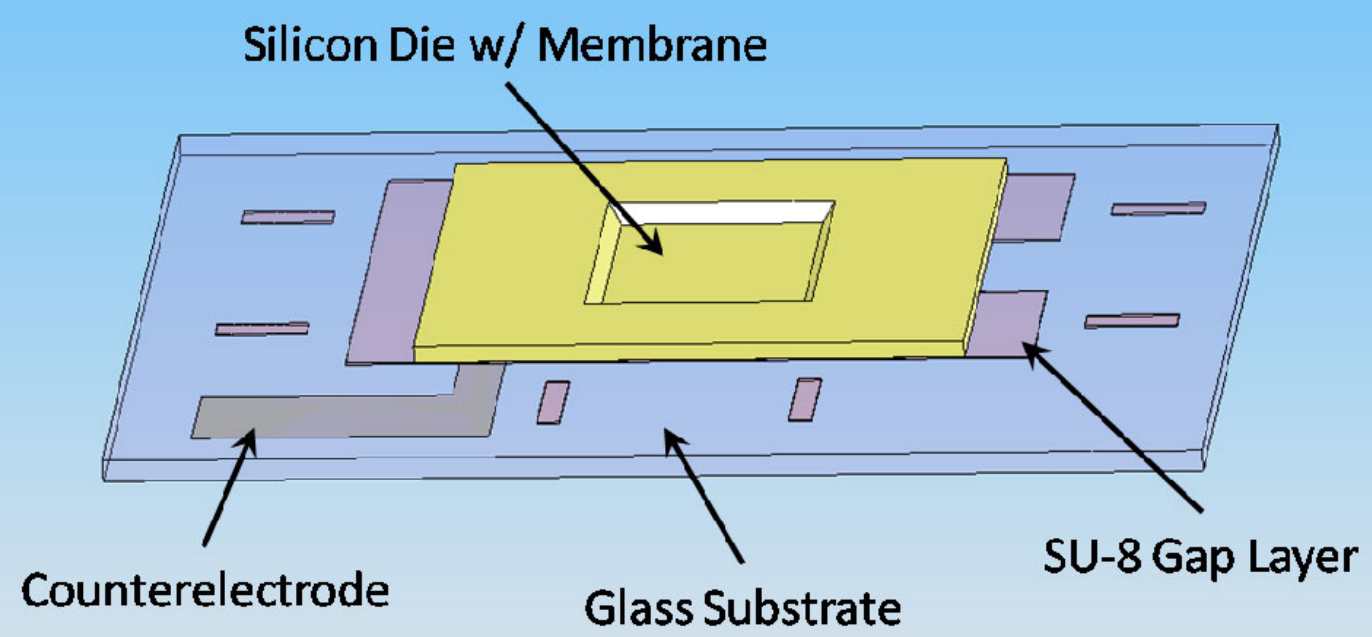

Figure 2.11: A 3-D perspective view of an assembled device.

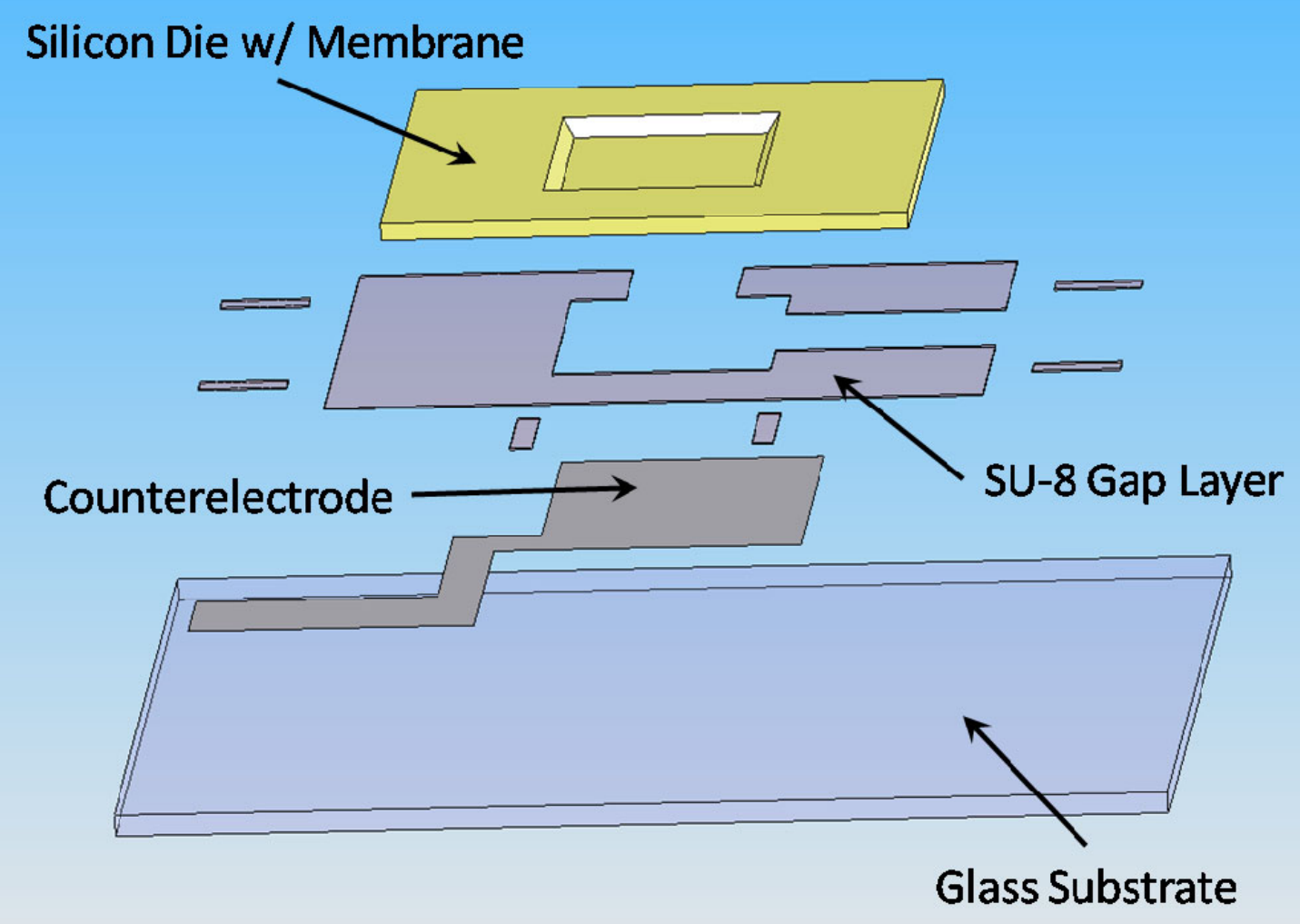

Figure 2.12: An exploded 3-D view of the final device. 


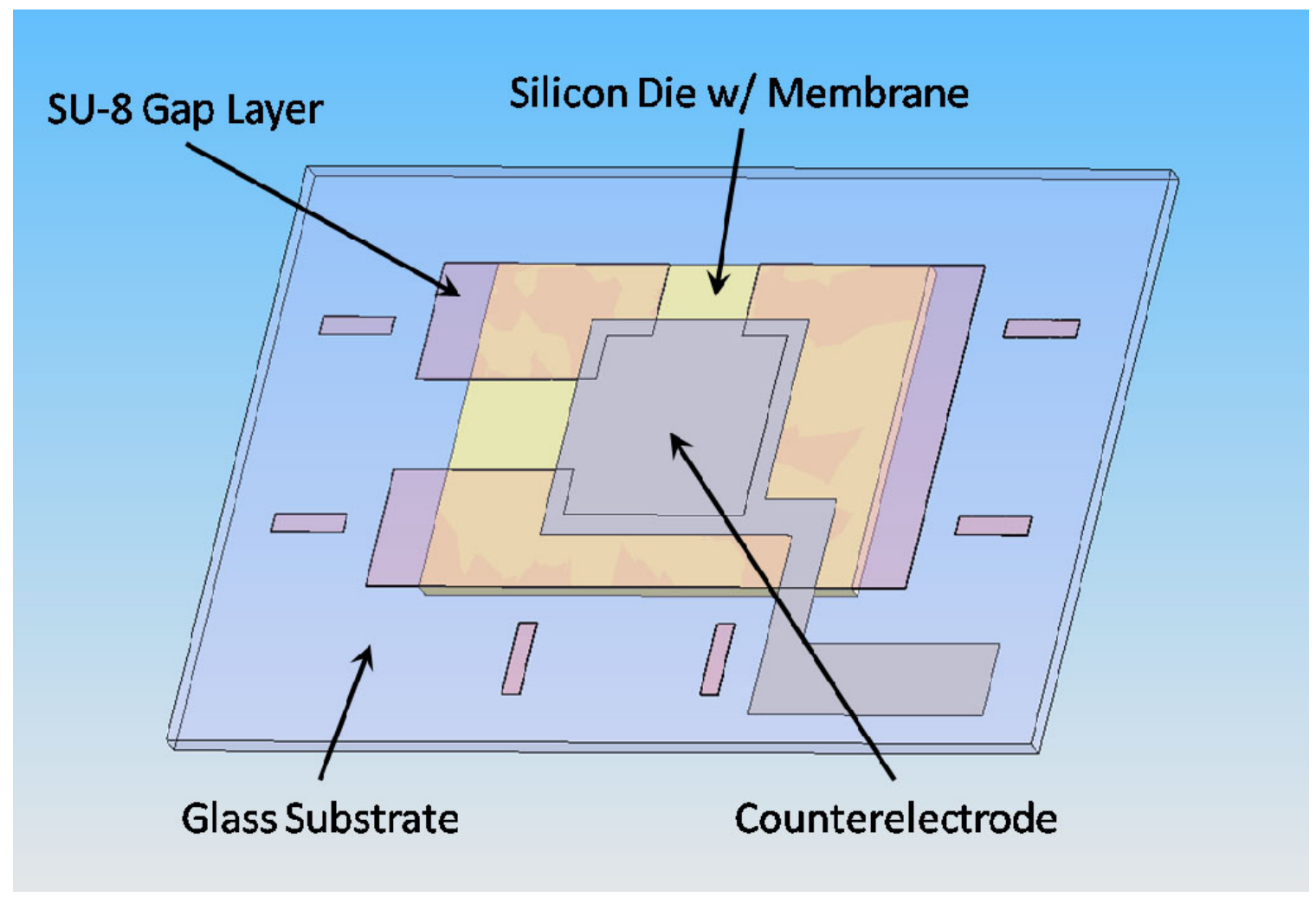

Figure 2.13: The back side of the final device. 


\section{SECTION 3: FABRICATION}

\subsection{Summary}

To fabricate the devices, double-sided polished (DSP) silicon wafers were heavily doped with boron on one side to create an etch-stop layer that significantly slows the etch rate when the etching reaches a region with a boron concentration of approximately $5 * 10^{19}$ atoms $/ \mathrm{cm}^{3}$ [21]. These wafers were then oxidized and patterned to create etch windows on the opposite side of the wafer from the heavily-doped side. The wafers were anisotropically etched through most of their thickness until the heavily-doped etch stop region was reached. Pyrex wafers were the coated with an aluminum film that was patterned to create counterelectrodes. These patterned pyrex wafers were then spincoated with SU-8, which was patterned to form an electrode gap layer. The silicon dies containing the membranes were separated and the membranes were aligned to their appropriate counterelectrodes. Finally, the silicon dies were bonded to the counterelectrode by thermally cross-linking the SU-8 to the silicon dies.

This section of the thesis will discuss the following fabrication steps:

- Wafer selection

- Thermal diffusion

- Diffusion characterization with D-SIMS

- Wet thermal oxidation

- Positive resist photolithography and patterning

- Wet anisotropic etching

- Counterelectrode physical vapor deposition

- Counterelectrode patterning

- $\mathrm{SU}-8$ resist processing

- Wafer bonding and die separation 
Additionally, key metrology steps will be discussed as well as the areas that impacted yield.

\subsection{Wafer Selection}

The substrate is of prime importance when fabricating MEMS devices. Many of the performance aspects of the final devices will be determined by the properties of the substrate, such as: Young's modulus, crystallographic orientation, defect and impurity concentration and background dopant concentration. In addition, if devices will be fabricated using deep etching, the geometry of the substrate becomes important. Specifically, the roughness of the wafer faces, the thickness and total thickness variation and the flatness of the wafer are crucial. Since the design required etching most of the way through the wafer leaving only a thin, uniform membrane on the opposite side, the wafers needed to be polished on both sides and have a low total thickness variation (TTV). The low-TTV specification was necessary because if a wafer was not uniformly thick, for example tapering from $410 \mu \mathrm{m}$ on one side to $400 \mu \mathrm{m}$ on the other side, after etching, the devices could vary in thickness by up to $10 \mu \mathrm{m}$. A TTV specification means that the difference in thickness between the thickest and thinnest sections of the wafer will not exceed the specified amount.

For these reasons, custom wafers were ordered from Silicon Quest International [33]. The specifications of these custom wafers are contained in Table 3.1. 


\begin{tabular}{|c|c|}
\hline Orientation and Size: & $100 \mathrm{~mm}$ silicon wafers, (100) orientation \\
\hline Growth: & Standard Czrochalski method \\
\hline Resistivity: & $10-20 \Omega$-cm, N-type (phosphorus doped) \\
\hline Polishing: & Double-sided polished \\
\hline Thickness: & $400 \pm 10 \mu \mathrm{m},<4 \mu \mathrm{m}$ TTV \\
\hline
\end{tabular}

Table 3.1: Custom low-TTV wafer specifications for deep-etching applications.

Prior to any processing these wafers had a surface roughness of less than $10 \mathrm{~nm}$ RMS, which is approximately the detection limit of the stylus profilometer used to measure surface roughness.

\subsection{Thermal Diffusion}

As mentioned in the previous section, a heavily boron-doped region of silicon has been shown to act as an etch-stop in wet anisotropic etching processes. This proved crucial to the fabrication process as it was the main method of defining the thickness of the membranes. Common methods of introducing high dopant concentrations into silicon include ion implantation and thermal diffusion. Thermal diffusion is a relatively simple, low-cost method of doping and is the only technique available in the Cal Poly Microfabrication facility. As discussed in the previous section, a process was designed to introduce the highest possible concentration of boron into the silicon wafer. This process was based on the 5-hour thermal diffusion at $1100^{\circ} \mathrm{C}$ discussed in the Design section.

Fabrication started with cleaning the custom double-sided polished wafers ordered from Silicon Quest Int'l using a typical piranha and buffered oxide etchant (BOE) process. Piranha is a solution of $90 \%$ sulfuric acid and $10 \%$ hydrogen peroxide, heated to $70^{\circ} \mathrm{C}$. Piranha acts as a chemical oxidizer and removes any organic contaminants on the wafers. 
As an oxidizer, it also grows a thin oxide film on the silicon that must be removed. The BOE solution is an off-the-shelf product called "Buffer HF Improved" from Transene Company [34], and is a mixture of hydrofluoric acid and a fluoride $\mathrm{pH}$ buffer. BOE rapidly etches silicon dioxide (and other materials) and leaves a clean, bare silicon surface for further processing.

After 10 minutes in the piranha solution, the wafers were dipped in BOE for approximately 1 minute to remove the oxide, rinsed in de-ionized (DI) water and then placed on a hot plate at $150^{\circ} \mathrm{C}$ to drive off any adsorbed water. Next, the wafers were spin-coated with a spin-on boron dopant source. To do this, each wafer was loaded into a Laurell Technologies WS-400B-6NPP spin-coater, $3 \mathrm{~mL}$ of Filmtronics B-155 spin-on dopant was dispensed in the center of the wafer and the wafer was spun using the twostep program obtained from Filmtronics and described in Table 3.2

\begin{tabular}{|c|c|c|}
\hline Step & Spin Speed (RPM) & Time $(\mathrm{sec})$ \\
\hline 1 & 300 & 300 \\
\hline 2 & 3000 & 3000 \\
\hline
\end{tabular}

Table 3.2: The two-step spin program used to coat wafers with spin-on dopant.

The Filmtronics B-155 spin-on dopant is a solution of ethyl alcohol and a boroncontaining species. During spin-coating, the liquid dispensed on the wafer is spread out and thinned into a flat, uniform film with a thickness that can be controlled by varying the spin speed. After spin-coating the spin-on dopant, the wafers were baked on a hot plate at $150^{\circ} \mathrm{C}$ for 5 minutes to harden the film and the solvent. 
After the spin-coating process was complete, the wafers were loaded into a quartz diffusion boat in pairs with the dopant-coated sides of the wafers in each pair facing each other, as shown in Figure 3.1 below.

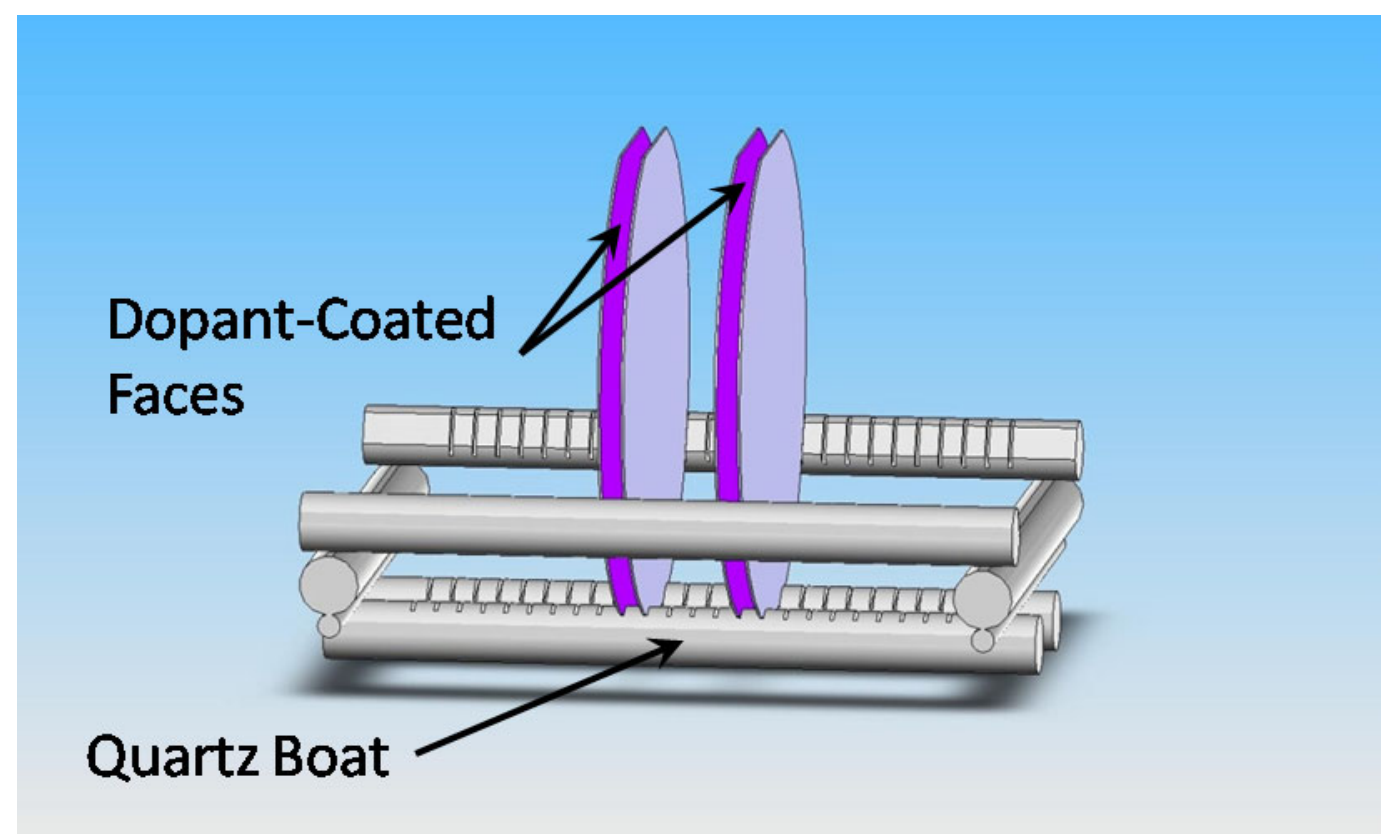

Figure 3.1: Orientation of the wafers in the boat prior to thermal diffusion.

The wafers were oriented with the coated sides facing inward to improve doping uniformity. The thermal diffusion was performed in a Thermco Mini-Brute MB-80 horizontal tube furnace at $1100^{\circ} \mathrm{C}$, with process gas flowing along the axis of the tube as shown in Figure 3.2. 

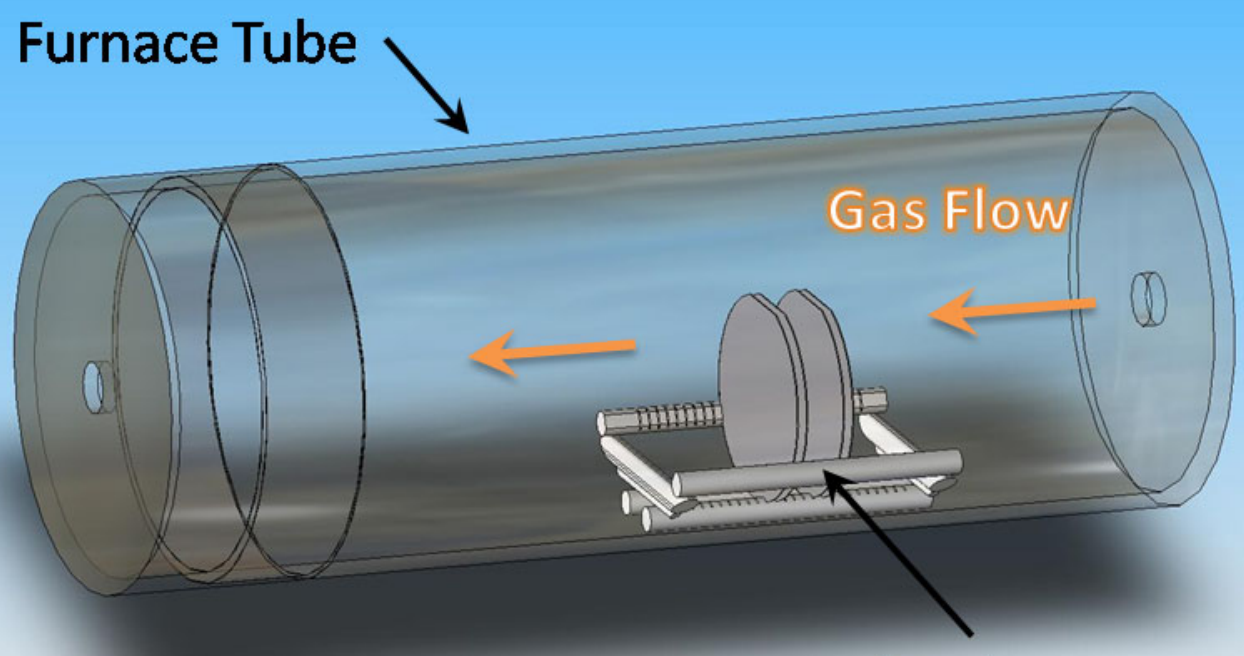

Wafers in Boat

Figure 3.2: Orientation of the tube furnace, wafer boat and gas flow (not to scale).

All process gases were ultra-high purity (UHP, 99.999\% pure or better) and were supplied by Airgas West. The thermal diffusion process is described in Table 3.3.

\begin{tabular}{|l|l|l|}
\hline Process Step & Temperature & Gas Flow \\
\hline 1) Insert Wafers & RT & None \\
\hline 2) Warm-up & $\mathrm{RT}-200^{\circ} \mathrm{C}$ & None \\
\hline 3) Warm-up & $200-500^{\circ} \mathrm{C}$ & $\mathrm{UHP} \mathrm{O}_{2}, 5 \mathrm{~L} / \mathrm{min}$ \\
\hline 4) Warm-up & $500-1100^{\circ} \mathrm{C}$ & $\mathrm{UHP} \mathrm{N}_{2}, 5 \mathrm{~L} / \mathrm{min}$ \\
\hline 5) Soak $-4: 30 \mathrm{~h}: \mathrm{mm}$ & $1100^{\circ} \mathrm{C}$ & $\mathrm{UHP} \mathrm{N}_{2}, 5 \mathrm{~L} / \mathrm{min}$ \\
\hline 6) Soak $-0: 30 \mathrm{~h}: \mathrm{mm}$ & $1100^{\circ} \mathrm{C}$ & $\mathrm{UHP} \mathrm{O}_{2}, 5 \mathrm{~L} / \mathrm{min}$ \\
\hline 7) Shutoff & $1100^{\circ} \mathrm{C}$ & $\mathrm{UHP} \mathrm{O}_{2}, 5 \mathrm{~L} / \mathrm{min}$ \\
\hline 7) Cool-down & $1100-500^{\circ} \mathrm{C}$ & $\mathrm{UHP} \mathrm{O}_{2}, 5 \mathrm{~L} / \mathrm{min}$ \\
\hline 8) Cool-down & $500^{\circ} \mathrm{C}-\mathrm{RT}$ & None \\
\hline 9) Remove Wafers & $\mathrm{RT}$ & None \\
\hline
\end{tabular}

Table 3.3: Thermal diffusion process designed to heavily dope silicon wafers with boron to create an etch-stop layer.

The wafers were inserted at room temperature and allowed to heat up to minimize thermal shock. The warm-up and cool-down steps took approximately 60 minutes each 
and were ignored in the diffusion calculations. The temperature was held constant during the soak steps and the total soak time was determined by the limits on the furnace control systems. The purpose of the oxygen gas flow during ramp-up was to oxidize any hydrocarbons still remaining in the spun-on dopant and to prevent hydrogen and carbon impurities from diffusing into the silicon. The ramp-up and "soak" portions were performed under an inert gas to prevent oxidation; oxide growth during the diffusion would act as a barrier to boron diffusion, reducing the concentration of boron in the wafers. The last half-hour of the "soak" and the ramp-down were performed under oxygen to oxidize any remaining elemental boron on the wafer and to facilitate removal of the borosilicate glass which forms on the surface of the wafer. The wafers were allowed to furnace-cool to minimize thermal shock.

\subsection{Diffusion Characterization with D-SIMS}

After the 5-hour pre-dep diffusion, dynamic secondary-ion mass spectroscopy (D-SIMS) was used to characterize the boron concentration profile in the silicon. This work was supported by the Materials Research Facility Network within the Materials Research Laboratory at the University of California, Santa Barbara, which is a Materials Research Science and Engineering Center supported by the National Science Foundation. A DSIMS sample wafer was prepared by thermal diffusion under processing conditions identical to those used to fabricate the device wafers. The boron concentration profile was measured at the center and also at the edge of this test wafer to determine if the boron concentration was consistent throughout. Figure 3.3 below shows the boron concentration as a function of depth into the silicon, indicating that a boron concentration 
of $5^{*} 10^{19}$ atoms $/ \mathrm{cm}^{3}$ was obtained at a depth of $\sim 3.5 \mu \mathrm{m}$ and not $1.3 \mu \mathrm{m}$ as predicted in Section 2.

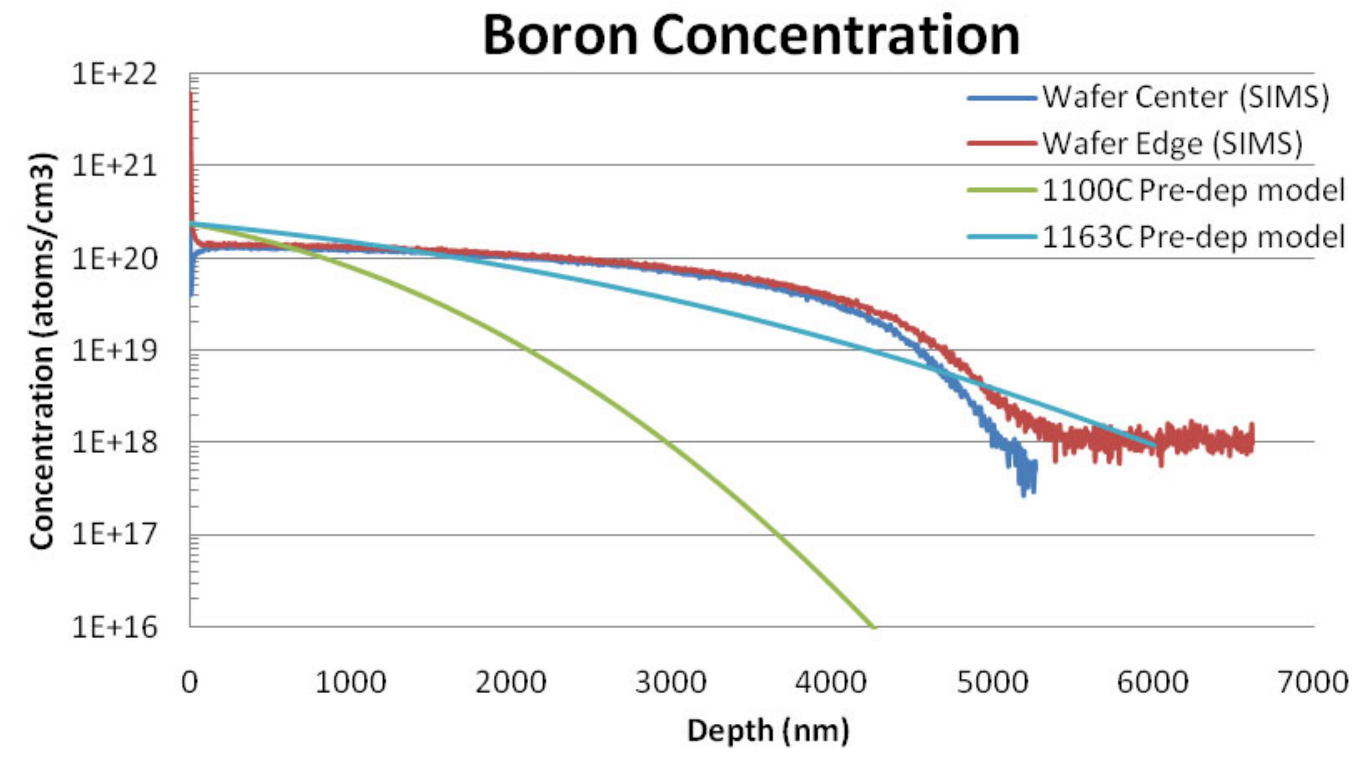

Figure 3.3: Boron concentration profiles after thermal diffusion.

The difference between the boron concentration at the center and edge of the wafer could be attributed to a slightly faster heating of the edge of the wafer inside the diffusion furnace. These D-SIMS results indicate that the samples received a much higher dopant dose than predicted by diffusion calculations. Included in Figure 3.3 is a curve indicating the predicted concentration profile resulting from a 5-hour pre-deposition thermal diffusion at $1100^{\circ} \mathrm{C}$. The disagreement between this predicted profile and the profile measured by D-SIMS could be the result of a higher diffusion temperature. The actual dopant dose was computed by integrating the area under the curve in Figure 3.3 and was found to be a factor of two greater than the dose predicted by the pre-deposition model. Eq. (3.1) gives the dopant dose $Q_{0}$ as a function of the diffusion coefficient $D$, the diffusion time $t$, and the surface dopant concentration $C_{S}$. 


$$
Q_{0}=\frac{2}{\sqrt{\pi}} C_{S} \sqrt{D t}
$$

This measured dose was used to back-calculate the actual diffusion coefficient and the corresponding diffusion temperature, which was calculated to be approximately $1163^{\circ} \mathrm{C}$. The expected dopant concentration profile resulting from a 5-hour pre-dep at $1163^{\circ} \mathrm{C}$ is also shown in Figure 3.3, and this curve is in closer agreement with the profile measured by D-SIMS. This suggests that the temperature in the furnace during diffusion was higher than the intended temperature of $1100^{\circ} \mathrm{C}$.

\subsection{Wet Thermal Oxidation}

The purpose of this thermal oxidation step was to grow a passivating film on the silicon that would act as an etch mask. This film would later be patterned using a standard photolithography process to define the etch windows which would be used to create the etch pit on the back side of the wafer, forming the thin membrane. Thermal oxides are commonly used as etch masks in silicon anisotropic etching, however, they exhibit some finite etch rate in most etchants. The ratio of the etch rate of the silicon to the mask material, in this case silicon dioxide, is referred to as the selectivity of the etchant and should be as high as possible to avoid removing the etch mask before the silicon etch is complete.

Etch rates for silicon dioxide in $\mathrm{KOH}$ and TMAH vary significantly based on etchant temperature, concentration and the technique used to grow the oxide; however, for comparable temperature and etchant concentrations the etch rate of thermally grown oxide is generally higher in $\mathrm{KOH}$ than TMAH [25]. In contrast to the electrochemical 
etching of silicon, silicon dioxide etching in alkaline solutions is thought to be purely chemical in nature and has been shown to depend linearly on hydroxide concentration below approximately 6.2 moles of hydroxide per liter of solution, and depend on the square of the water concentration above 6.2 molar concentration of hydroxide [27]. The difference in oxide etch rates for comparable concentrations of $\mathrm{KOH}$ and TMAH can be explained by the different mass fraction of hydroxide ions for the two solutions. The hydroxide ion has a molar mass of $17 \mathrm{~g} / \mathrm{mol} ; \mathrm{KOH}$ has a molar mass of $56 \mathrm{~g} / \mathrm{mol}$ and thus a hydroxide mass fraction of $30.4 \%$, while TMAH has a molar mass of $91 \mathrm{~g} / \mathrm{mol}$ and a hydroxide mass fraction of $18.7 \%$. Therefore, for comparable etchant concentrations there is a higher concentration of hydroxide ions in $\mathrm{KOH}$ solutions than TMAH solutions. Thus, below a hydroxide concentration of approximately $6.2 \mathrm{~mol} / \mathrm{L}$ (corresponding to 35 wt $\% \mathrm{KOH}$ or $56.9 \mathrm{wt} \% \mathrm{TMAH}), \mathrm{KOH}$ solutions etch oxide faster than solutions of comparable concentrations of TMAH. This was one of the factors which contributed to the decision to etch with TMAH. It was still prudent, however, to have a reasonably thick oxide etch mask and, therefore, the target oxide thickness was $500 \mathrm{~nm}$.

Thermal oxides are typically grown either with a "dry" or a "wet" oxidation process. In either case, the oxidation proceeds in a three step process: diffusion of the oxidizing species from the environment to the wafer surface, diffusion of the oxidizing species through any oxide film present on the wafer and, finally, reaction of the oxidizer with the silicon surface [35]. 
In "dry" oxidation, pure oxygen flows into the furnace and reacts with the silicon to form $\mathrm{SiO}_{2}$. In "wet" oxidation, oxygen as well as water flows into the furnace to react with the silicon; however, the oxide grows much faster in the presence of water vapor for two reasons. The first reason is that water is a smaller molecule than oxygen, and so it diffuses through the oxide layer to the silicon surface faster than oxygen, increasing the growth rate. The second reason is that hydroxyl groups are incorporated into the growing oxide film, increasing the disorder of the film and creating a more open structure, which allows the oxidizing species to diffuse more rapidly [22].

The Deal-Grove model can be used to accurately predict oxide thickness based on growth parameters [35]. This model is described by the equation:

$$
X_{o x}(t)=\frac{A}{2}\left(\sqrt{1+\frac{4 B(t+\tau)}{A^{2}}}-1\right)
$$

Here, $X_{o x}$ is the oxide thickness in microns, the constants $A$ and $B$ for wet oxidation at $1050^{\circ} \mathrm{C}$ are $0.292 \mu \mathrm{m}$ and $0.35 \mu \mathrm{m}^{2} / \mathrm{hr}$, respectively, $t$ is the oxidation time in hours and $\tau$ is a factor used to account for any oxide present on the wafer at the start of the oxidation step. Using this equation, it was determined that a 68 -minute wet oxidation at $1050^{\circ} \mathrm{C}$ should yield a 500nm thick oxide layer.

After the wafers had cooled from the thermal diffusion, they were removed from the diffusion furnace, the boron-doped side was scribed with a code to identify each wafer and the boron-doped side and they were dipped in BOE for approximately 15 minutes to remove the borosilicate glass that formed during the diffusion. As a convention, the 
boron-doped side of the wafer is referred to as the back side. The wafers were cleaned in piranha solution for 10 minutes to remove any organic contaminants and to oxidize any remaining elemental boron, followed by another BOE dip for one minute. The wafers were then loaded into a quartz oxidation boat and oriented so that the front sides of the wafers were facing the gas inlet nozzle inside the furnace. The boron-doped wafers were book-ended with dummy wafers in preparation for wet oxidation as shown in Figure 3.4.

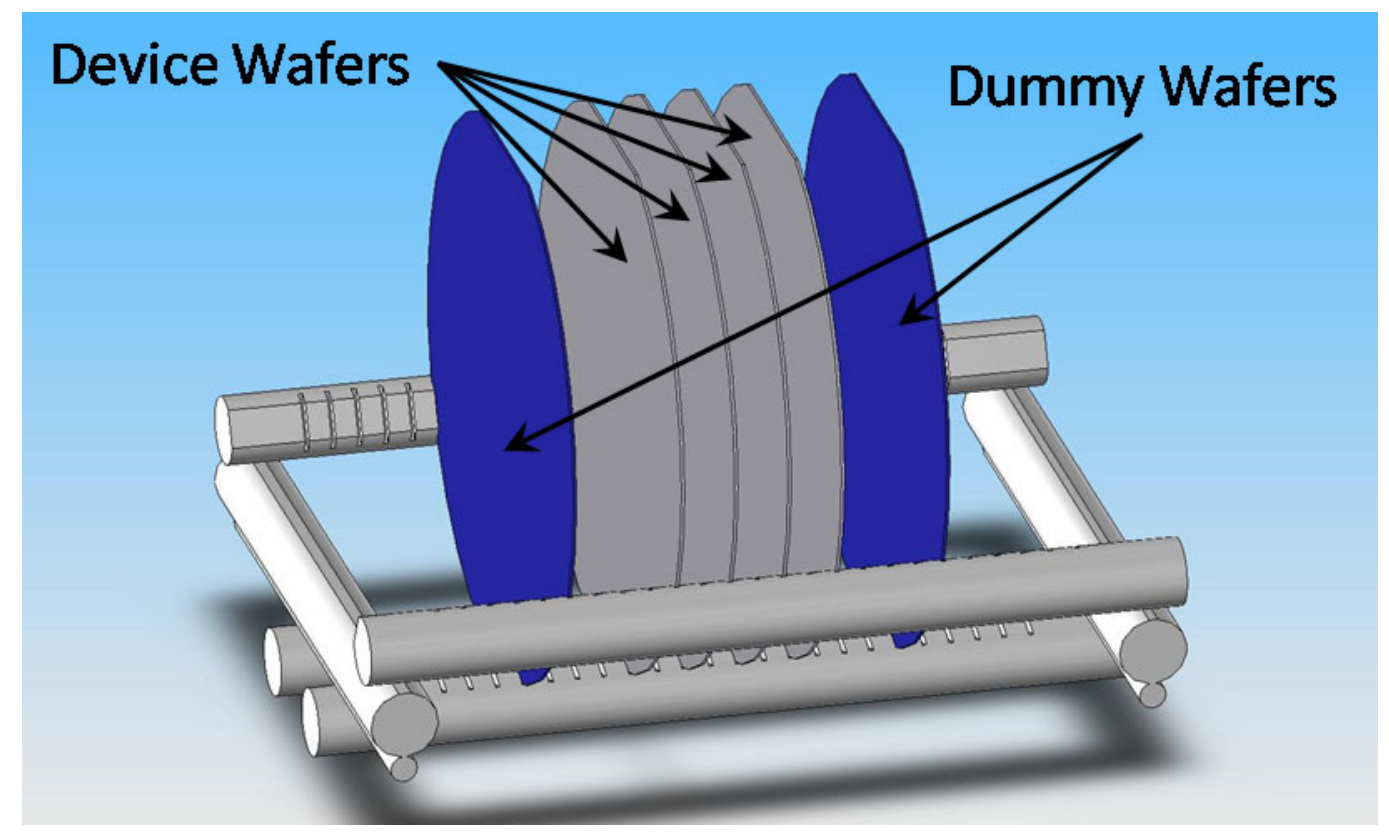

Figure 3.4: Device wafers book-ended by dummy wafers, loaded in a quartz boat.

The dummy wafers shown in Figure 3.4 were non-device wafers with an existing oxide layer and were included to create a non-turbulent gas flow around the device wafers. Table 3.4 lists the process parameters that were used to grow a 500nm-thick wet thermal oxide. 


\begin{tabular}{|l|l|l|}
\hline Process Step & Temperature & Gas Flow \\
\hline 1) Pre-heat furnace & RT $-850^{\circ}$ & None \\
\hline 2) Insert Wafers & $850^{\circ} \mathrm{C}$ & UHP N $_{2}, 5 \mathrm{~L} / \mathrm{min}$ \\
\hline 3) Warm-up & $850-1050^{\circ} \mathrm{C}$ & UHP N $_{2}, 5 \mathrm{~L} / \mathrm{min}$ \\
\hline 4) Soak - 1:08 h:mm & $1050^{\circ} \mathrm{C}$ & Wet UHP O $_{2}, 5 \mathrm{~L} / \mathrm{min}$ \\
\hline 5) Cool-down & $1050-500^{\circ} \mathrm{C}$ & UHP N $_{2}, 5 \mathrm{~L} / \mathrm{min}$ \\
\hline 6) Cool-down & $500^{\circ} \mathrm{C}-\mathrm{RT}$ & None \\
\hline 7) Remove Wafers & RT & None \\
\hline
\end{tabular}

Table 3.4: Thermal oxidation process designed to grow a 700nm wet oxide.

The oxidation tube furnace (Thermco Mini-Brute MB-80) was pre-heated to $850^{\circ} \mathrm{C}$ and 5 $\mathrm{L} / \mathrm{min}$ UHP nitrogen was flowed into the furnace. The boat was loaded into the oxidation furnace at $850^{\circ} \mathrm{C}$ at a rate of approximately $0.5 \mathrm{~cm} / \mathrm{sec}$ to avoid thermally shocking the wafers. Once the boat was fully inserted into the furnace, the temperature was ramped up to $1050^{\circ} \mathrm{C}$. When the furnace reached $1050^{\circ} \mathrm{C}$, the nitrogen was stopped and $5 \mathrm{~L} / \mathrm{min}$ UHP oxygen was flowed into the furnace through a bubbler containing $95^{\circ} \mathrm{C}$ DI water. Bubbling the oxygen through hot water introduces water vapor into the gas feed which accelerates the oxidation process, as discussed earlier in this section. After flowing wet oxygen for 68 minutes, the power to the furnace was shut off and the wafers were allowed to furnace-cool to $500^{\circ} \mathrm{C}$ under $5 \mathrm{~L} / \mathrm{min}$ UHP nitrogen. When the temperature reached $500^{\circ} \mathrm{C}$, the nitrogen flow was stopped and the wafers were allowed to cool to room temperature in air.

\subsection{Positive Resist Photolithography and Patterning}

When the wafers had cooled, a Filmetrics F20 thin-film measurement system was used to measure the thickness of the oxide on the front of the wafers and to verify that each had an oxide film approximately 500nm thick. Next, the wafers were brought into the spin- 
coating station and placed on a hot-plate at $150^{\circ} \mathrm{C}$ to drive off adsorbed water prior to spin-coating. The wafers were loaded individually into a Laurell Technologies WS400B-6NPP spin-coater with the front side facing up, and $2 \mathrm{~mL}$ of Microchem MCC Primer 80/20 was dispensed on each wafer to promote resist adhesion. Each wafer was spun at 3000 RPM until the primer appeared to evaporate completely, then $2 \mathrm{~mL}$ of Rohm-Haas Microposit S1813 positive photoresist was dispensed into the center of each wafer and each wafer was spun using the 3-step process described in Table 3.5.

\begin{tabular}{|c|c|c|}
\hline Step & Spin Speed (RPM) & Time (sec) \\
\hline 1 & 300 & 5 \\
\hline 2 & 500 & 10 \\
\hline 3 & 3000 & 30 \\
\hline
\end{tabular}

Table 3.5: 3-step spin process for positive photoresist application.

The purpose of the first two spin steps was to spread the photoresist out and cover the wafer surface, while the final spin step planarized the photoresist and achieved the desired film thickness. The photoresist films resulting from this spin-coating procedure were typically $1 \mu \mathrm{m}$ thick. After spin-coating, the wafers were soft-baked on a hot-plate for 1 minute at $90^{\circ} \mathrm{C}$ to drive off most of the solvent in the resist.

After being coated with positive photoresist, each wafer was exposed in a Canon PLA501FA aligner tool with a high-pressure mercury arc lamp using the etch window lithography mask and a light integral of 5. This light integral corresponded to an exposure time of approximately 20 seconds and an exposure dose of approximately 32 $\mathrm{mJ} / \mathrm{cm}^{2}$. After exposure, the resist was developed in Microchem MF-CD-26 positive 
resist developer at room temperature for 2 minutes, which removed the photoresist exposed by the mask. After being rinsed in DI water, the wafers were hard-baked on a hot-plate at $150^{\circ} \mathrm{C}$ for 1 minute to completely drive off the solvent from the resist and make the resist more resistant to chemical attack during subsequent processing. The back side of each wafer was then spin-coated with photoresist using the same spin-coating process. The purpose of this back side coat was to protect the oxide on the back side during the oxide etch step to create the etch windows on the front side. After coating the back side, the resist was soft-baked and hard-baked using the same processes as the prior spin-coating step.

Once the back side had been coated, the exposed oxide was etched in BOE for 10 minutes to expose the bare silicon which would later be etched to create the membranes. After etching in BOE the wafers were rinsed in DI water, dried and immersed in Shipley Microposit Remover 1165 at $60^{\circ} \mathrm{C}$ for 10 minutes to remove the hard-baked photoresist. The resist removal step was followed by a 10-minute piranha clean to remove any remaining organic contaminants prior to wet anisotropic etching.

\subsection{Wet Anisotropic Etching}

As mentioned previously in this paper, wet anisotropic etching can be performed using a variety of alkaline solutions, including $\mathrm{KOH}$ and TMAH. Preliminary experiments were performed to compare the etch properties of aqueous TMAH (obtained as electronic grade $25 \mathrm{wt} \% \mathrm{TMAH}$ from Transene Company) and aqueous $\mathrm{KOH}$ (obtained in pellet form, $85 \%$ by weight, $99.99 \%$ purity from Alfa Aesar) solutions, and it was determined 
that the etch selectivity of wet thermal oxide to silicon in $\mathrm{KOH}$ was too high for $\mathrm{KOH}$ to be used as the etchant. It was found that the silicon-to-oxide selectivity of $14 \mathrm{wt} \% \mathrm{KOH}$ at $75^{\circ} \mathrm{C}$ was less than $500: 1$ and that the oxide etch rate at these conditions was $\sim 100$ $\mathrm{nm} / \mathrm{hr}$; in contrast, the oxide-to-silicon selectivity of $25 \mathrm{wt} \% \mathrm{TMAH}$ at $85^{\circ} \mathrm{C}$ was found to be in excess of 6000:1 and the oxide etch rate was found to be $\sim 10 \mathrm{~nm} / \mathrm{hr}$. With an etch selectivity of less than 500:1, over $1000 \mathrm{~nm}$ of thermal oxide would have to be grown for the oxide mask to survive the entire etch process. Growing an oxide film of that thickness is both time-consuming and expensive. In addition, TMAH is less toxic than $\mathrm{KOH}$ [25] and is supplied in ready-to-use formulations. For these reasons, TMAH was selected as the anisotropic etchant.

The surface roughness of monocrystalline silicon surfaces etched with TMAH was found to generally decrease with increasing TMAH concentration because at lower TMAH concentrations the evolved hydrogen bubbles have a longer adherence time on the silicon surface and act as pyramid-forming micro-masks $[25,31,36]$. This relationship was verified by experimentation. Wafers were etched separately in $2.5 \mathrm{wt} \%$ and $15 \mathrm{wt} \%$ TMAH at $90^{\circ} \mathrm{C}$, and both conditions resulted in a surface roughness of approximately 2 $\mu \mathrm{m}$ RMS as measured with a stylus profilometer. Another wafer was etched in $25 \mathrm{wt} \%$ $\mathrm{TMAH}$ at $90^{\circ} \mathrm{C}$, resulting in a surface roughness of less than $20 \mathrm{~nm}$ RMS measured with a stylus profilometer. Thus, a high TMAH concentration facilitates achieving smooth etched surfaces. TMAH is commonly available in concentrations up to $25 \mathrm{wt} \%$, so this etchant concentration was chosen. 
The etch temperature of $85^{\circ} \mathrm{C}$ was chosen because the etch rate at that temperature was both high enough to be practical and low enough to be somewhat forgiving of overetching. The [100] etch rate of $25 \mathrm{wt} \% \mathrm{TMAH}$ at $90^{\circ} \mathrm{C}$ was found to be approximately $50 \mu \mathrm{m} / \mathrm{hr}$, while the etch rate decreased to approximately $35 \mu \mathrm{m} / \mathrm{hr}$ at $85^{\circ} \mathrm{C}$. Thus, etching at $85^{\circ} \mathrm{C}$ through several hundred microns of silicon is still practical for laboratory applications and it affords better tolerance to over-etching.

The etchant temperature and concentration was held constant in a reflux-condensing etch vessel heated with two independent heating systems. While the concentration of the etchant was not measured, no observable change in the fluid level inside the etch vessel was detected over the 1-week time periods during which the same etchant solution was used. To reduce evaporative losses, the etchant was stored in a sealed high-density polyethylene container when not in use. The etch vessel was a 4000mL Kimble Kimax beaker that had been fitted with a custom-built high-density polyethylene (HDPE) watercooled lid with a water-cooled vent tube. The water coolant was recirculated at approximately 1 gallon/minute through an external Lytron Kodiak RC009J03BE2 chiller set at $12^{\circ} \mathrm{C}$. The lid and vent tube allowed the vessel to be exposed to atmospheric pressure, thus preventing any pressure buildup. The water-cooling caused liquid evaporating from the etchant to condense and fall back into the solution, keeping the concentration of the etchant constant. The etch vessel was heated from below by a Barnstead-Thermolyne 7" square hot-plate. In addition, a flexible foil heater (Minco HK5177R58.8L12A) was wrapped around the etch vessel and powered by an Omega CSi32K temperature controller. 
This two-heater system was designed because it was determined that the hot plate was incapable of maintaining a stable etchant temperature. Specifically, the etchant temperature was found to fluctuate by several degrees hour-to-hour, causing fluctuations in the etch rate up to $5 \mu \mathrm{m} / \mathrm{hr}$ which resulted in unpredictable etch depths and, frequently, over-etching wafers until the membranes were completely etched away. It was determined that this fluctuation was due to the primitive temperature controller inside the hot-plate. It was thought that adding an additional heater driven by a proportionalintegral-derivative (PID) temperature controller would provide more precise control over the temperature. This supplemental temperature controller would measure the temperature of the etchant directly through a submerged thermocouple, periodically applying power to the foil heater to maintain a constant temperature. To prevent the supplemental temperature controller from competing with the hot plate temperature controller, the hot plate was set at a lower temperature and the additional controller was used to raise the temperature to the desired level. The temperature of the etchant was monitored with another submerged thermocouple and an independent thermometer. This etch vessel is shown in Figure 3.5 and diagrammed in Figure 3.6. 


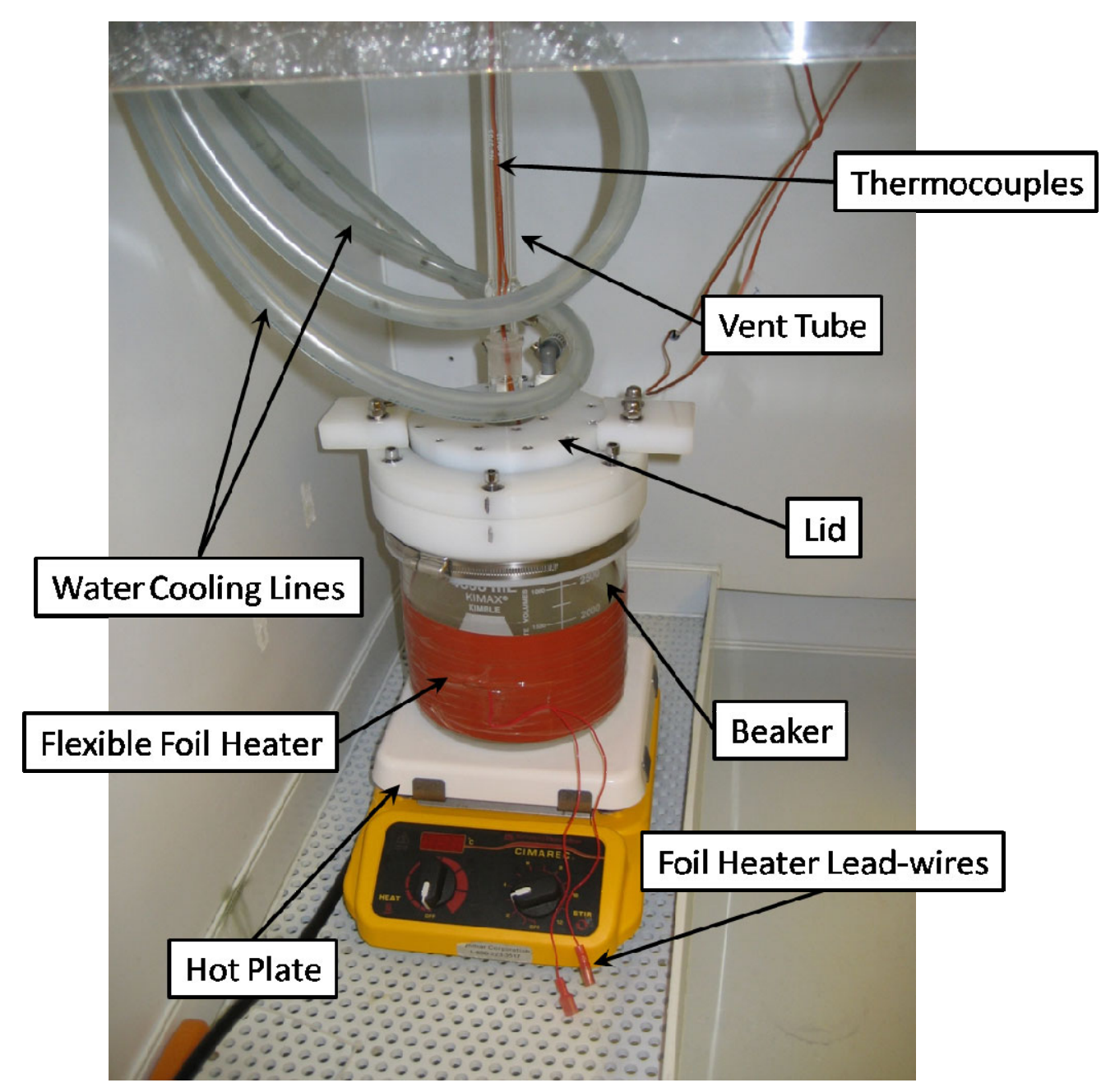

Figure 3.5: The reflux-condenser etch vessel. 


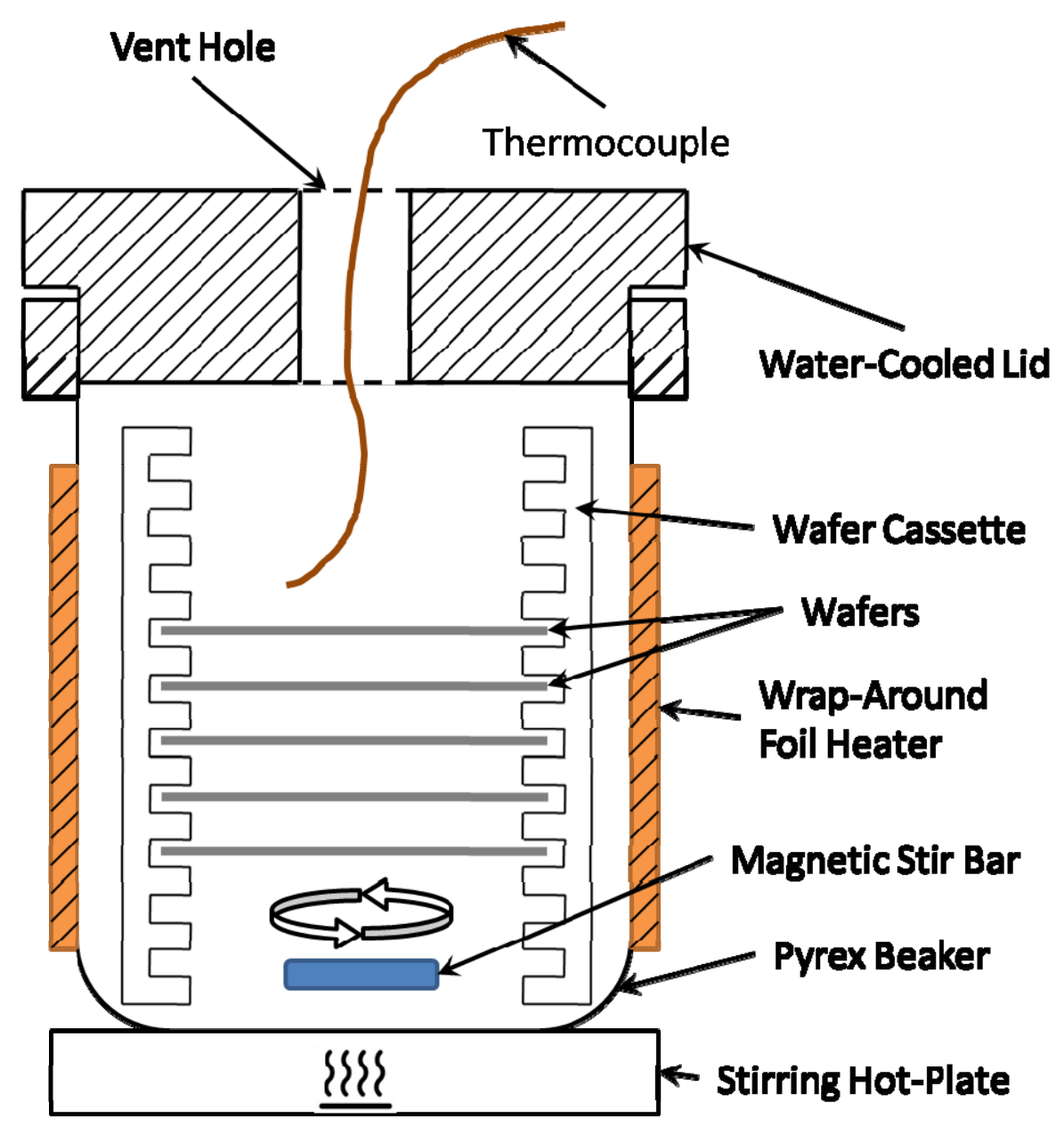

Figure 3.6: A diagram of the reflux-condenser etch vessel.

It was determined that a stable temperature of $85^{\circ} \mathrm{C}$ could be achieved by setting the hot plate at $130^{\circ} \mathrm{C}$ and the Omega temperature controller at $85^{\circ} \mathrm{C}$. Without the additional temperature controller, a hot-plate setting of $130^{\circ} \mathrm{C}$ raised the temperature of the etchant to approximately $70^{\circ} \mathrm{C}$. When this additional temperature controller and heater were incorporated into the etch vessel, a very stable etchant temperature was achieved that fluctuated by no more than $\pm 0.2^{\circ} \mathrm{C}$ at steady-state. A constant stir bar rotational speed of 500 RPM was used for all anisotropic etching processes. The temperature was assumed to be uniform throughout the etch vessel. 
Immediately prior to etching in TMAH, the wafers were dipped in BOE for 15 seconds to remove the native oxide and then loaded into a Teflon cassette and immersed in a solution of $25 \mathrm{wt} \% \mathrm{TMAH}$ at $85^{\circ} \mathrm{C}$. After 8.5 hours, the average etch depth was $294 \mu \mathrm{m}$ with a standard deviation of $1.4 \mu \mathrm{m}$ across 23 measurement points on 2 wafers. This corresponded to an etch rate of $34.6 \mu \mathrm{m} / \mathrm{hr}$. Etch depth measurements were made with an Ambios XP-1 stylus profilometer with a $440 \mu \mathrm{m}$ range. The profilometer was calibrated using a built-in software calibration routine by measuring a $100 \mu \mathrm{m}$ step-height standard supplied by Ambios. The etch depth was measured by driving the stylus from the surface of the wafer down into an etch pit and measuring the height difference between the surface of the wafer and the etch pit as shown in Figure 3.7. The step height repeatability of the profilometer was better than $0.1 \%$.

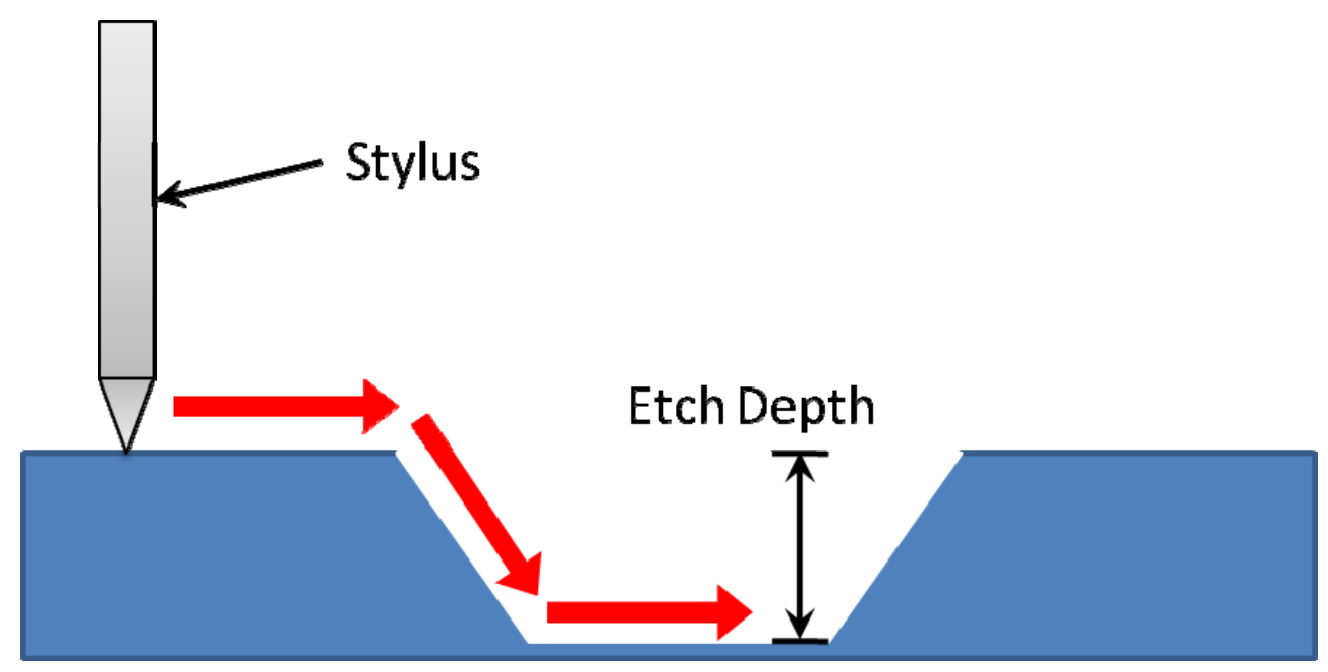

Figure 3.7: Measuring the etch depth with a stylus profilometer. 


\subsection{Etched Surface Morphology}

After the initial 8.5 hours of etching, cratering was observed on many of the (100) etched surfaces as shown in Figure 3.8. The craters appeared to be randomly distributed, were typically less than $100 \mu \mathrm{m}$ in diameter and were on the order of hundreds of nanometers to one micron deep. Larger craters were observed infrequently as shown in Figure 3.9. Additionally, a tightly-packed linear array of craters was observed in one etch pit, shown in Figure 3.10.

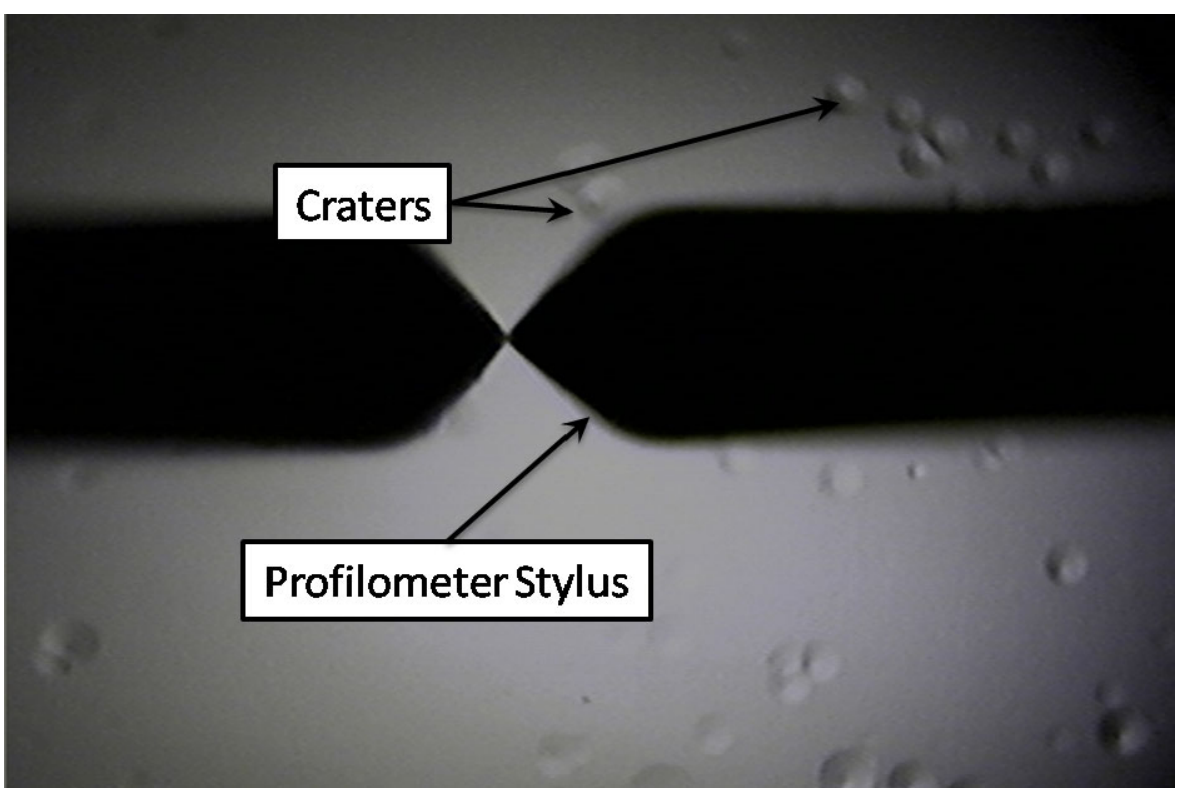

Figure 3.8: Crater-type defects observed on etched (100) surfaces. 


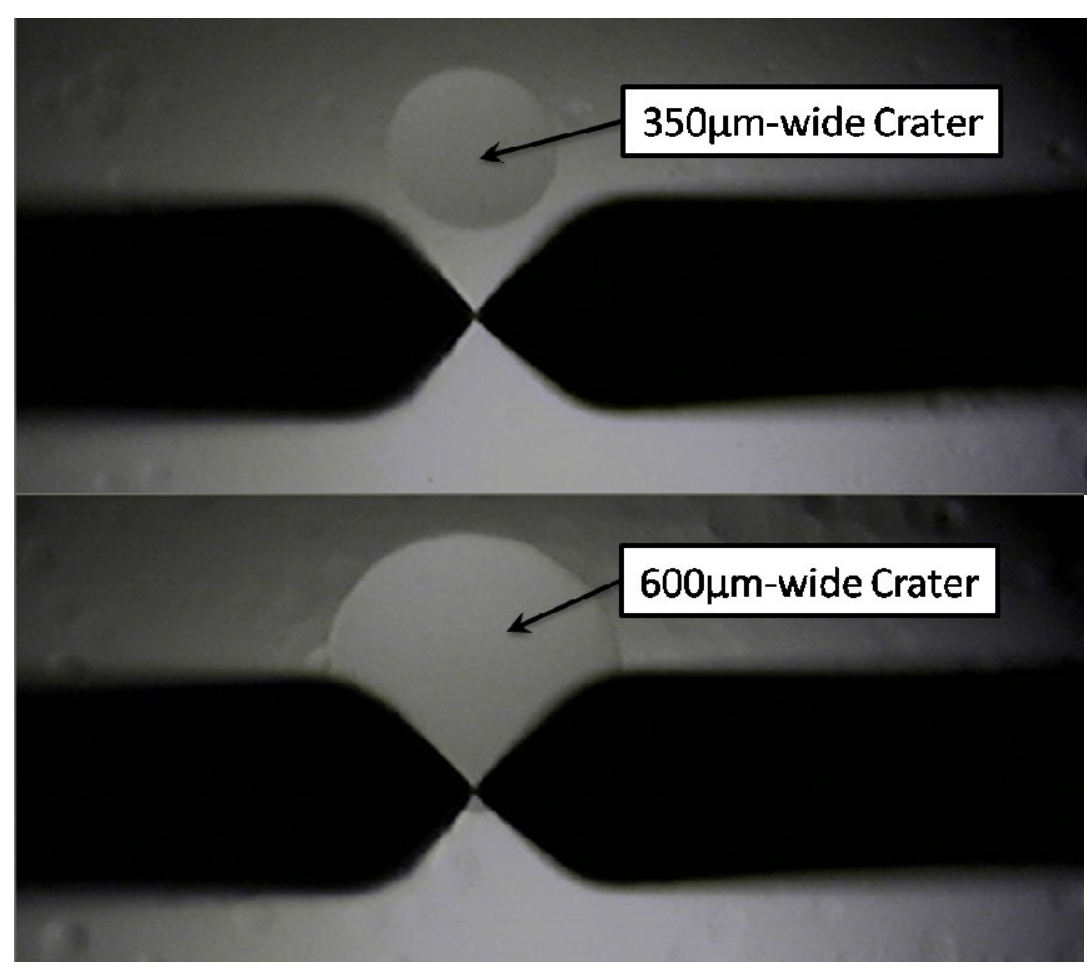

Figure 3.9: Unusually large crater defects.

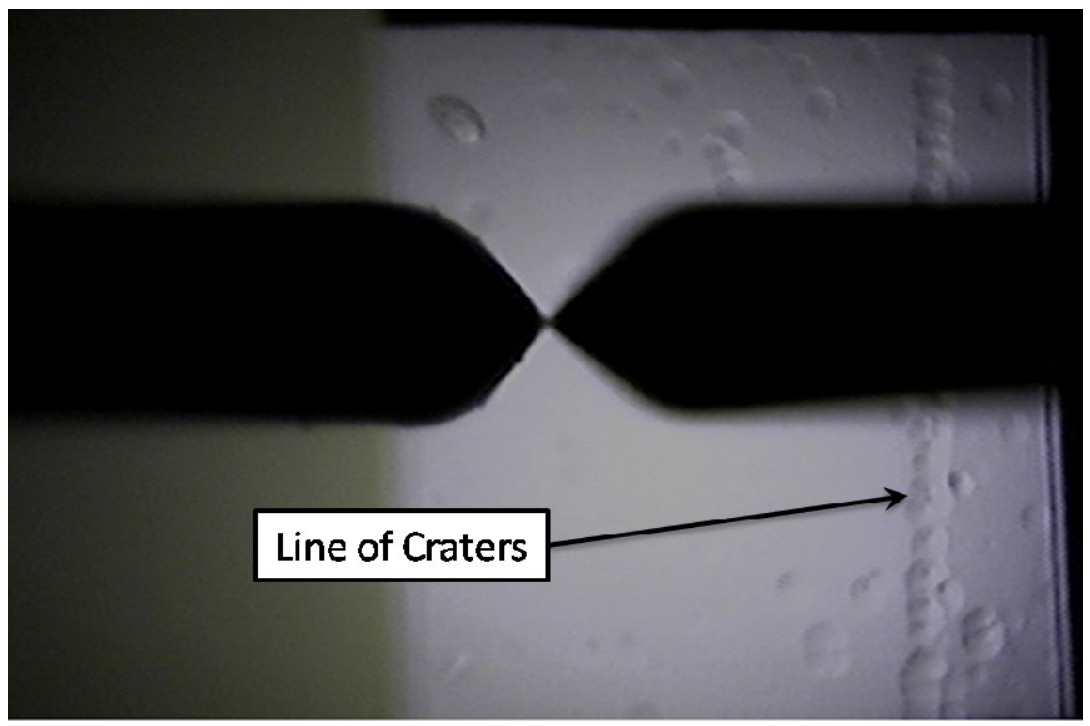

Figure 3.10: A line of craters extending across an etch pit.

Hillock-type surface morphological defects have been widely reported [30-31, 36-40]; however, these crater-type defects were not discussed in the literature. The mechanism of hillock formation is generally accepted to be localized micro-masking of the silicon 
surface by pinned gas bubbles or other particles which result in small pyramidal or coneshaped features protruding from the etched surface [39]. In contrast to pyramidal or hillock-type defects which protrude up from the etched surface, the defects observed here are smooth, circular craters extending down into the etched surface with elliptical profiles similar to that shown in Figure 3.11.

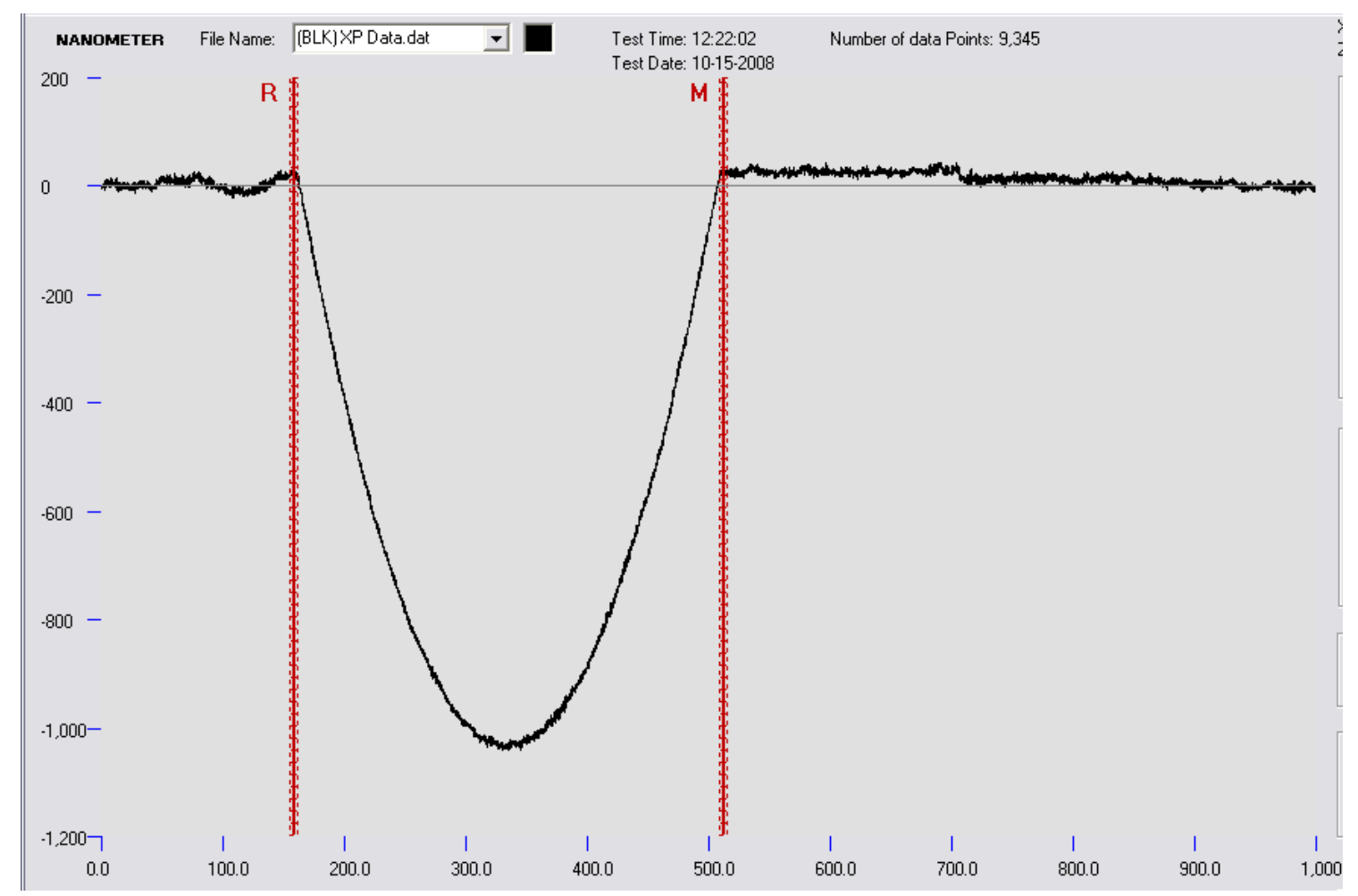

Figure 3.11: A profile scan representative of the shape of the crater defects. Horizontal axis units are microns, vertical axis units are nanometers.

One of two hypotheses could explain the cause of these craters. The first hypothesis is that these craters are located where common $\{111\} /<110>$ dislocation loops intersect the etching (100) surface. As discussed in Section 2.8, removing a silicon atom from the surface requires the destabilization and cleavage of backbone bonds which tie the surface atom to the bulk. The (111) plane is thought to etch slower than other planes because 
surface atoms on this plane have three backbone bonds which must be broken, in contrast to (100) surface atoms which have only two backbone bonds. It is well known that there is an elastic stress field around dislocation cores [41] and this elastic stress field could destabilize the backbone bonds of surface atoms close to the dislocation core, causing them to be removed from the surface faster than atoms far from the dislocation core. The elastic stress increases as the distance to the dislocation core is reduced and this stress gradient could cause atoms closer to the core to be removed more rapidly than those further from the core, causing the craters to be deeper at the dislocation core and accounting for the curved profile of the craters. It is well known that Czochralski-grown silicon wafers can have on the order of hundreds of dislocations per square centimeter due to the growth process [42]; these dislocations could account for the number of observed etch pits on the silicon (100) surfaces.

It is hypothesized that individual craters could occur where a dislocation line intersects the etched silicon surface at a non-parallel angle; uncharacteristically large craters, such as those shown in Figure 3.9, could be formed by a larger elastic stress field caused by the pile-up of multiple dislocations. Additionally, it is hypothesized that the line of craters shown in Figure 3.10 could be formed by a dislocation line running parallel to the etched surface or a linear array of dislocation lines normal to the surface caused by a dislocation source, e.g. a Frank-Read source [43].

A number of etches have been developed to delineate crystalline defects, such as dislocations, in single-crystal silicon wafers [44-48] and it is believed that these etches 
intentionally utilize a similar mechanism to delineate dislocation defects. These etches range in composition from solutions of nitric, acetic and hydrofluoric acids to mixtures of hydrofluoric acid and a complex chromium compound, but all yield etch pits at the intersection of dislocation lines and etched silicon surfaces. It is hypothesized that anisotropically etching single-crystal silicon can unintentionally achieve the same result. This hypothesis could be verified by obtaining (100) silicon wafers with varying concentrations of dislocations, quantifying the dislocation density in these samples, etching these samples in $25 \mathrm{wt} \% \mathrm{TMAH}$ and comparing the observed etch pit density to the measured dislocation density.

This dislocation-crater hypothesis was considered by others [49-50], however its validity was called into question because the craters should exhibit more of a cone shape if the cause was preferential etching of a dislocation core. It was thought that this cone shape would result because the dislocation core would continue to be etched preferentially to the bulk silicon, resulting in an initially shallow cone which became deeper as etching progressed. These craters do not appear to become deeper as etching progresses; rather, they exhibit a maximum depth on the order of a few hundreds of nanometers to one micron.

An alternate hypothesis exists which could explain the craters. This second hypothesis is that etching proceeds isotropically within the craters due to atomic vacancy and microscopic void defects incorporated into the silicon during the ingot growth process. The accumulation of atomic vacancies could cause a localized disruption of the crystal 
structure, causing the TMAH to etch the silicon isotropically. In Czochralski crystal growth, it has been well established that if the crystal is grown too quickly, vacancy point-defects can accumulate and form randomly distributed octahedral voids within the bulk silicon [51]. There are approximately $10^{14}-10^{15}$ entropically necessary atomic defects, both vacancy and self-interstitial, per cubic centimeter in silicon near its melting point at $1685 \mathrm{~K}$. If the growing ingot is pulled from the melt too quickly, as the crystal cools the slower-diffusing vacancy defects are kinetically prevented from recombining with their self-interstitial counterparts and will preferentially accumulate, nucleating into octahedral voids that reach approximately $100 \mathrm{~nm}$ in size. The self-interstitials, meanwhile, form extra half-planes of silicon atoms.

These defects remain in the silicon after the ingot is processed and are incorporated into the final wafers. The random distribution of these void/vacancy defects matches the random distribution of the craters and the relatively uniform size of the defects could explain the relative uniformity in the size of the craters. As etching continues the silicon surface containing the craters is etched away, exposing new defects which form new craters. It has been shown that similar circular etch craters can be intentionally caused by introducing nanometer-scale indentations into the surface of the silicon prior to the etching process [52]. The size and shape of these induced craters is similar to that of the craters observed in this work, suggesting that they are of the same nature. It was proposed that nanometer-scale indentations in the silicon surface become larger circular craters with time because the etch rates around the [100] direction are comparable. It is hypothesized that this mechanism would also cause octahedral voids to be etched into 
larger circular craters. If this hypothesis is correct, then the line of craters shown in Figure 3.10 could be created by a linear array of octahedral defects which nucleated along a dislocation line running parallel to the plane of the wafer. This hypothesis could be verified by obtaining low-defect-density wafers grown at a slower rate, etching the wafers, and comparing the observed crater density between the two types of wafers.

It should be noted that when no craters were situated on the stylus scan path, the etched (100) surfaces were found to be exceptionally smooth and exhibited surface roughness values on the order of 10nm RMS. Because the craters were randomly distributed over the etched surface as point-localized defects, the stylus profilometer produced inconsistent surface roughness measurements. If the scan profile passed through one or more craters, the reported RMS roughness value was on the order of $1 \mu \mathrm{m}$. If, however, the scan profile did not pass through any craters, the reported RMS roughness values were as low as $10 \mathrm{~nm}$. Since the craters are hypothesized to be caused by defects in the silicon wafer, the RMS surface roughness values for crater-free scan profiles are taken to be representative of the etching process.

It had been reported that adding isopropyl alcohol (IPA) to TMAH solutions had the effect of reducing surface roughness due to hillocks [37-38]. The proposed mechanism for surface roughness reduction hypothesizes that IPA molecules adsorb onto the etching silicon surface and inhibit the adsorption of water molecules and reactive hydroxide groups, causing etching to proceed less aggressively. The proposed mechanism hypothesized that IPA molecules have different adsorption affinities for silicon atoms 
with different surface bonding properties, i.e. silicon atoms on different crystallographic planes, and that the addition of IPA also impacts the anisotropy of the TMAH etchant. The most obvious effects of IPA addition are a reduction in the surface roughness of etched silicon surfaces and a slight reduction in the etch rate [38]. It was thought that the addition of IPA could have a similar effect on crater formation, so the $25 \mathrm{wt} \%$ TMAH solution was diluted with 10 vol\% IPA to attempt to reduce the surface roughness during the rest of the etch. The wafers were etched in this new TMAH/IPA solution at $85^{\circ} \mathrm{C}$ for 2.5 hours. After this 2.5 hour etch, the etch rate for the TMAH/IPA solution was measured to be $27.9 \mu \mathrm{m} / \mathrm{h}$, and it was determined that approximately 1.2 hours of further etching was necessary to form membranes.

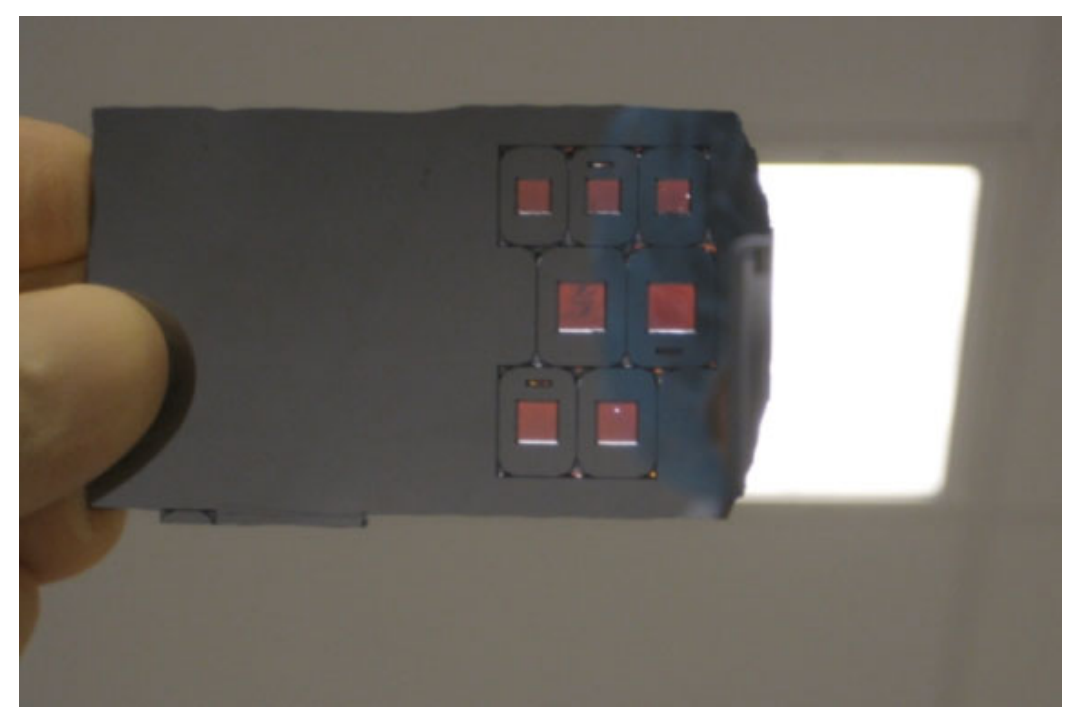

Figure 3.12: Membranes exhibiting optical translucency.

After the additional 1.2-hour etch, the wafers were removed and observed to be translucent towards the red end of the visible spectrum, as shown in Figure 3.12. There was no significant change in the cratering or the surface roughness observed on the 
etched (100) surfaces after the addition of IPA. If either of the two hypotheses presented in this section concerning the origin of the craters are correct, then the best way to eliminate the craters is to fabricate the membranes on low-defect-density silicon wafers.

In addition to the craters, the membranes were also bowed as shown in Figure 3.13, with more pronounced bowing on the larger membranes. This bowing was not directiondependent and the membranes could be freely popped in and out by simply touching the membrane or drying the wafer with a stream of compressed nitrogen gas, although more membranes exhibited concave bowing than convex bowing when observed from the etched side. The wafers were etched in BOE for 10 minutes to remove the oxide layer; some bowing was still present after the oxide was etched away.

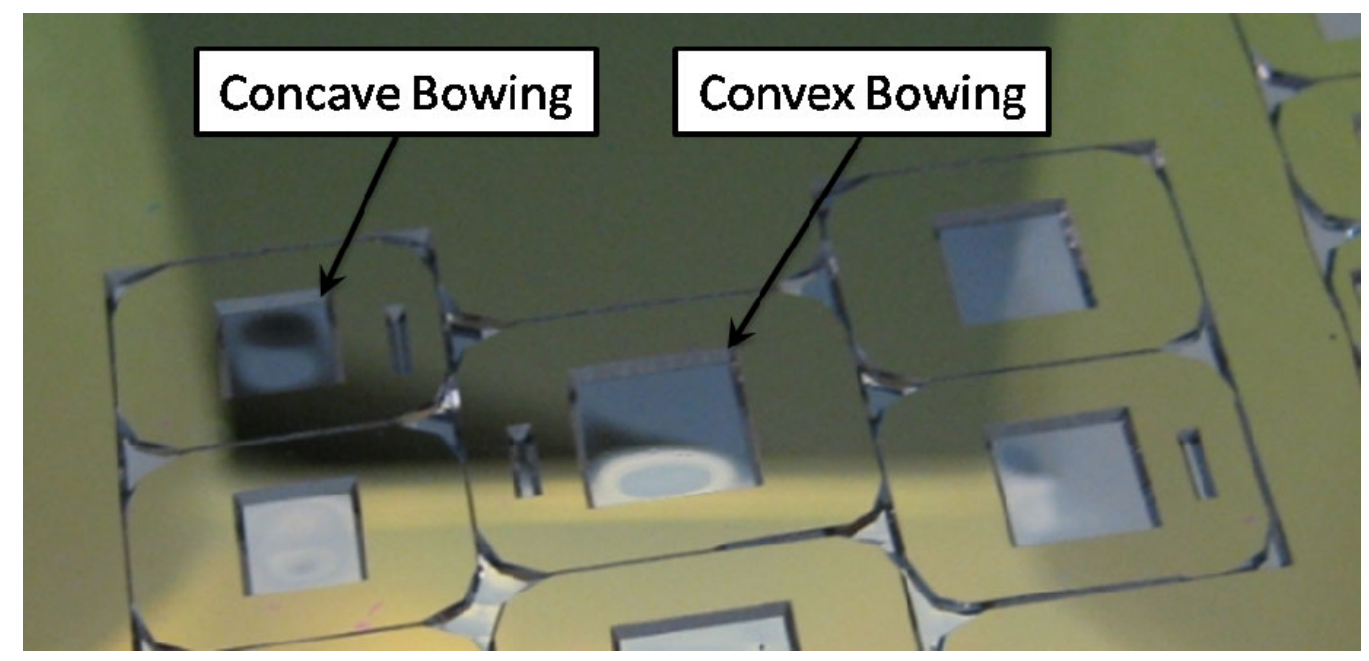

Figure 3.13: Membrane bowing after deep etching.

The bowing is believed to be caused by the growth of the thermal silicon oxide film on the wafer after the diffusion process [53]. It is well established that boron is a substitutional impurity in silicon and that a tensile residual stress is created when smaller- 
covalent-radius boron atoms replace silicon atoms at silicon lattice sites [54]. When a thermal oxide film is grown on the doped wafers at $1050^{\circ} \mathrm{C}$ it initially has no stress. However, because the thermal expansion coefficient of silicon dioxide $\left(0.35^{*} 10^{-6} \mathrm{~K}^{-1}\right)$ is much less than that of silicon $\left(4.5^{*} 10^{-6} \mathrm{~K}^{-1}\right)$, as the wafers cool from the oxide growth temperature the silicon experiences a greater contraction than the oxide film [55]. This thermal expansion coefficient mismatch causes a tensile stress in the silicon and a compressive stress in the oxide film. When an oxide grown at $1050^{\circ} \mathrm{C}$ cools to $800^{\circ} \mathrm{C}$ the magnitude of the stress in the oxide-silicon interface is approximately $133 \mathrm{MPa}$ [53], which is well above the flow stress of undoped silicon at that temperature [56]. Plastic deformation due to tensile stress occurs in the silicon at the oxide-silicon interface. When the membranes are released during the deep anisotropic etching process, they buckle because the bulk silicon no longer restrains the plastically deformed silicon. This could be verified by measuring the curvature of the doped side of the wafer after the doping process, and after thermal oxidation and removal of the thermal oxide film; no deep anisotropic etching would be required. The wafer should exhibit concave bowing after doping due to the substitutional boron tensile stress, and the wafer should exhibit convex bowing after oxidation and removal of the oxide film due to the plastic deformation upon cooling from the oxidation temperature. Because the observed wafer bowing due to thin-film residual stresses increases as the substrate thickness decreases, a thinner wafer could be used for this experiment to accentuate the curvature.

If this hypothesis is verified, this bowing could be eliminated by one of two methods. One method would be to etch away plastically deformed material from the front of the 
wafer after releasing the membranes and removing the thermal oxide; this method would result in a thinning of the membrane which would have to be compensated for during the initial membrane release etch. The second method would be to prevent plastic deformation in the silicon by masking the front and back sides of the wafer with a material other than thermally grown silicon dioxide. Instead of a thermal oxide, a sputtered metallic film such as $80 / 20$ nickel/chromium, gold, or nickel [57], or a chemical-vapor-deposited dielectric such as silicon dioxide or silicon nitride could be used to mask the silicon.

Using the profilometer, the etch depth was measured to be $401.5 \mu \mathrm{m}$ corresponding to an etch rate of $29.1 \mu \mathrm{m} / \mathrm{hr}$ for the last 3.7 hour etch in the TMAH/IPA solution at $85^{\circ} \mathrm{C}$. The standard deviation of the etch depth was $1.8 \mu \mathrm{m}$ across nearly a dozen membrane cavities on two different wafers. The profilometer could not be used to accurately measure the thickness of the entire wafer because of an air gap of unknown thickness between the wafer and the glass plate below the wafer. A Vernier micrometer was used to measure the thickness of the wafers and the thickness was found to range from 401 to $406 \mu \mathrm{m}$, which is consistent with the $400 \pm 10 \mu \mathrm{m}$ thickness specification from the wafer supplier. However, an exact membrane thickness could not be obtained by subtracting the etch pit depth from the wafer thickness due to a lack of calibration between the two instruments.

Membrane thickness uniformity could not be quantified with the stylus profilometer because the variation in thickness of the wafers, i.e. TTV of $4 \mu \mathrm{m}$, could mask any change in etch pit depth caused by the membrane thickness. However, the translucency of the 
membranes was visually inspected and was observed to be uniform for all membranes, so the membranes were assumed to be uniformly thick. This qualitative judgment was considered to be valid because prior experimentation showed that the visible-spectrum optical transmission of silicon increased in both intensity and spectral range with decreasing membrane thickness as shown in Figure 3.14. Therefore, if the optical transmission appeared uniform across the wafer then the thickness should also be uniform. Several membranes were separated from their die and their thicknesses were measured directly using the profilometer, as shown in Figure 3.15. Using this technique, thickness values of approximately $7 \mu \mathrm{m}$ were obtained. This thickness measurement was influenced by the bowing of the membranes and the air gap between the membrane and the glass slide that they were placed on; therefore, this measurement was assumed to be accurate to within approximately $1 \mu \mathrm{m}$. This thickness value was larger than anticipated; however, the etching was not continued because of the risk of damaging the wafer or membranes. Since these membranes were thicker than anticipated, it was expected that the maximum deflection would be significantly less than the design deflection unless the device design was modified. This extra thickness was compensated for by reducing the electrode gap from $67 \mu \mathrm{m}$ to $40 \mu \mathrm{m}$ which kept the deflection factor $1 /\left(d^{2} h^{3}\right)$ from Eq. (2.9) approximately constant. 


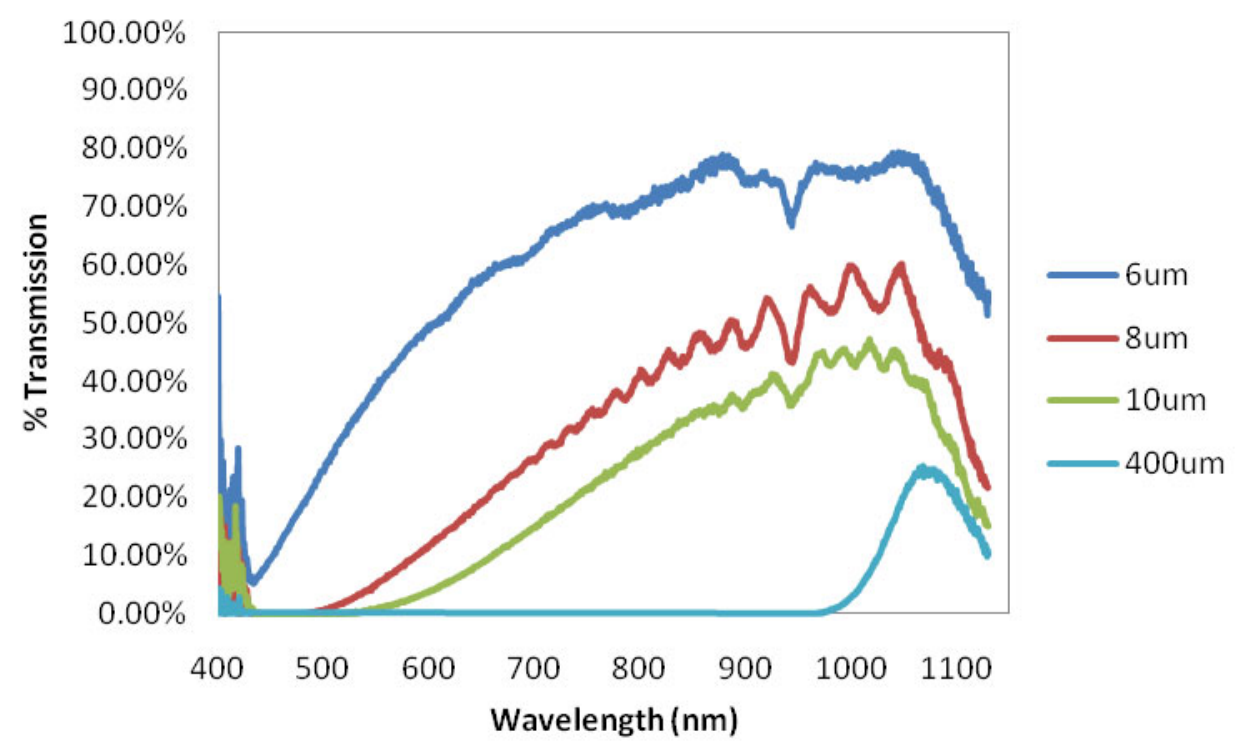

Figure 3.14: Membrane optical transmission as a function of membrane thickness. Legend contains approximate membrane thicknesses in microns.

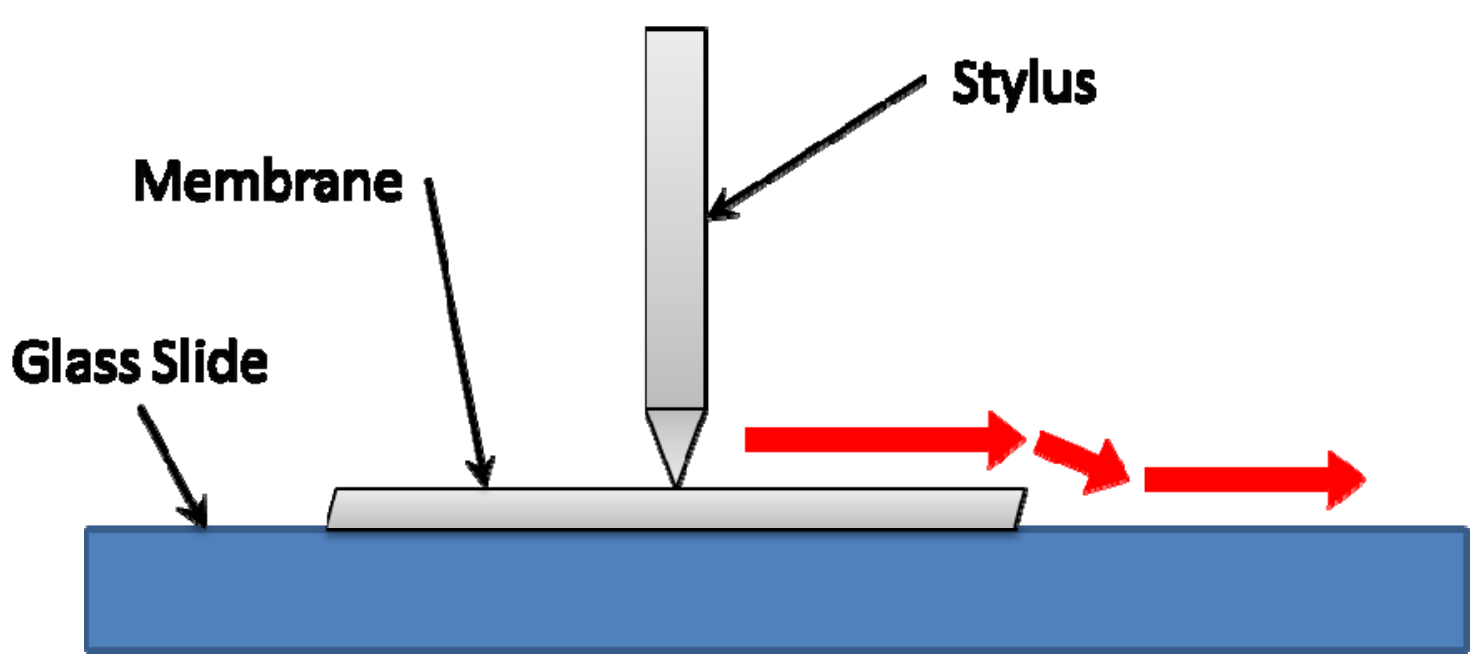

Figure 3.15: Measuring membrane thickness with the stylus profilometer.

It must be noted that this work does not represent a thorough evaluation of the etch-stop properties of heavily boron-doped silicon. Because the membranes were measured to be approximately $7 \mu \mathrm{m}$ thick when removed from the etchant, the boron concentration of the silicon exposed to the etchant was, at most, approximately $1 * 10^{18}$ atoms $/ \mathrm{cm}^{3}$ (Figure 3.3 ). 
By all accounts, this boron concentration was insufficient to cause a significant reduction in etch rate. It is therefore unknown whether the final membrane thickness was determined by the etch-stop characteristics of the silicon, the exact timing of the etching, or a combination of the two. However, an experiment to determine the efficacy of the boron etch-stop could be performed one of two ways. First, the open circuit potential (OCP) of the wafer could be monitored during the etching process. It has been established that the OCP of heavily-boron-doped silicon is shifted anodically by approximately $0.5 \mathrm{~V}$ from the OCP of lightly- or un-doped silicon when measured against a saturated calomel electrode [58]. When the OCP displays an anodic shift on the order of half a volt, the heavily-doped region has been reached. The sample could then be removed from the etchant and the membrane thickness could be determined. The second way to determine the efficacy of the etch-stop would be to etch multiple, identical wafers or wafer fragments at once and remove them sequentially, at regular intervals, at the end of the etching process. The thickness of the membranes could be measured and plotted as a function of total etching time; an abrupt change in the slope of this plot would identify the onset of etch-stop behavior and the final membrane thickness.

\subsection{Counterelectrode Physical Vapor Deposition}

After the membranes were etched, the counterelectrode and electrode gap were fabricated. $100 \mathrm{~mm}$, double-sided polished, $500 \mu \mathrm{m}$ thick Pyrex 7740 wafers were cleaned in piranha solution for 10 minutes. These wafers were then covered with an aluminum film via sputtering in a Torr $\mathrm{CrC}-150$ sputtering system. The sputtering process parameters are listed in Table 3.6. 


\begin{tabular}{|c|c|}
\hline Pumpdown time: & 30 minutes \\
\hline Argon base pressure: & $5 \mathrm{mT}$ \\
\hline Power: & $50 \mathrm{~W}$ \\
\hline Time: & 14 minutes \\
\hline Film Thickness: & $200 \mathrm{~nm}$ \\
\hline
\end{tabular}

Table 3.6: Aluminum sputtering parameters.

The film thickness was measured to be approximately 200nm using the XP-1 profilometer, which was calibrated using a $1 \mu \mathrm{m}$ step-height standard supplied by Ambios.

\subsection{Counterelectrode Photolithography and Patterning}

After depositing the aluminum film, the wafers were spin-coated with positive photoresist using the same process as the oxide patterning process. The photoresist was patterned with the counterelectrode lithography mask. This pattern was transferred into the aluminum by etching with Transene Aluminum Etchant Type A at $50^{\circ} \mathrm{C}$ until the exposed aluminum was removed, which took approximately 2 minutes. The photoresist was removed using the removal process discussed earlier in this section. The patterned aluminum counterelectrodes are shown in Figure 3.16. 


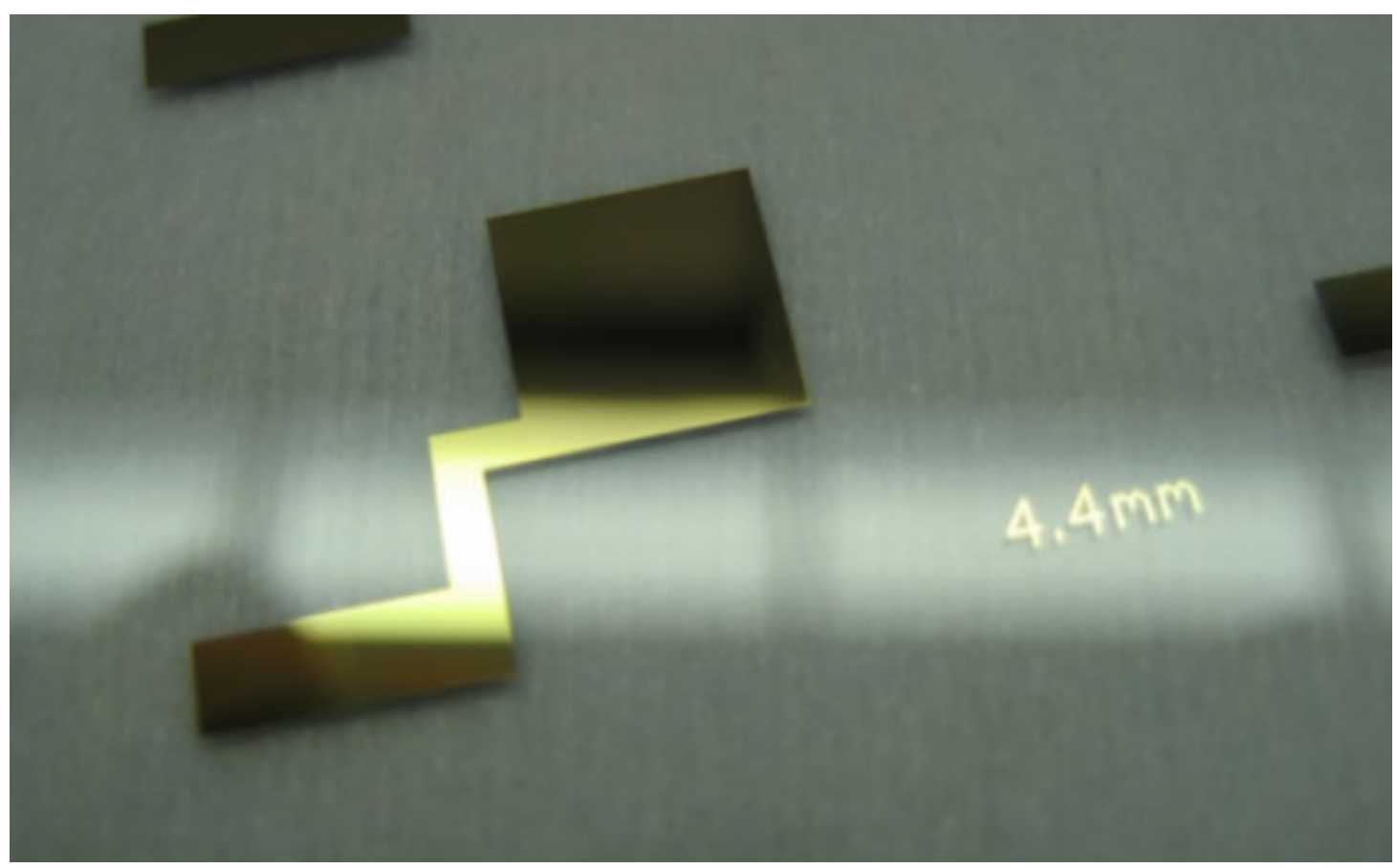

Figure 3.16: An etched aluminum counterelectrode on a Pyrex substrate.

\subsection{SU-8 Negative Photoresist Processing}

The next processing step to fabricate counterelectrodes was to put down a layer of SU-8 onto the Pyrex wafer which would both set the electrode gap and bond the silicon die to the Pyrex wafer. SU-8 is an epoxy-based negative photoresist produced by Microchem. It is available in a range of viscosities and can be spin-coated to yield films with a wide range of thicknesses [59]. When SU-8 is exposed to UV light, a strong photo-acid is generated which thermally cross-links the polymer chains during a subsequent baking step. Thus, the areas of the film which are exposed become permanent, while the unexposed regions do not cross-link and are removed during development.

To prepare the Pyrex wafers for SU-8 application, they were rinsed with acetone and DI water and placed on a hot plate at $150^{\circ} \mathrm{C}$ for several minutes to drive off any adsorbed 
water. The wafers were loaded into a Laurell Technologies WS-400-8N/L spin coater and approximately one tablespoon of room-temperature Microchem SU-8 2050 was dispensed in the center of the wafer. The wafer was spun using the spin program described in Table 3.7.

\begin{tabular}{|c|c|c|}
\hline Step & Spin Speed (RPM) & Time (sec) \\
\hline 1 & 250 & 10 \\
\hline 2 & 500 & 5 \\
\hline 3 & 800 & 5 \\
\hline 4 & 2650 & 40 \\
\hline
\end{tabular}

Table 3.7: SU-8 coating spin-program designed to yield $\sim 40 \mu \mathrm{m}$ film.

Based on thickness data collected from prior uses of SU-8 2050 in the Microfabrication facility, a final spin speed of 2650 RPM was chosen to achieve an SU-8 film thickness of approximately $40 \mu \mathrm{m}$. After spin-coating, the wafers were removed and placed on a flat, level surface for approximately 5 minutes at room temperature to allow the edge bead to dissipate. The SU-8 was soft-baked on a hot-plate using the process described in Table 3.8 .

\begin{tabular}{|c|c|c|}
\hline Step & Temperature $\left({ }^{\circ} \mathrm{C}\right)$ & Time $(\mathrm{min})$ \\
\hline 1 & 55 & 2 \\
\hline 2 & 85 & 6 \\
\hline
\end{tabular}

Table 3.8: Soft-bake times and temperatures for $\sim 40 \mu \mathrm{m}$-thick SU-8.

This two-step process is recommended by Microchem and is designed to slowly drive off the solvents in the SU-8 without forming a hardened "skin" on the outside of the resist and without prematurely cross-linking the polymer. 
After the soft-bake, the SU-8 was exposed in the aligner for a light integral of 12 , corresponding to a 48 -second exposure and an exposure dose of $78 \mathrm{~mJ} / \mathrm{cm}^{2}$. This is approximately half of the Microchem-recommended exposure dose of $150 \mathrm{~mJ} / \mathrm{cm}^{2}$, however, it was sufficient to expose the resist and transfer the mask pattern. After exposure, the exposed wafers were baked on a hot-plate using the process described in Table 3.9.

\begin{tabular}{|c|c|c|}
\hline Step & Temperature $\left({ }^{\circ} \mathrm{C}\right)$ & Time $(\mathrm{min})$ \\
\hline 1 & 55 & 2 \\
\hline 2 & 85 & 5 \\
\hline
\end{tabular}

Table 3.9: Post-exposure bake times and temperatures for $\sim 40 \mu \mathrm{m}$-thick SU-8.

As mentioned, this post-exposure bake caused the photo-generated acid to thermally cross-link the polymer chains in the exposed areas of the film. The wafers were developed in Microchem SU-8 Developer for 2 minutes with agitation to dissolve the uncross-linked resist. After developing, the wafers were rinsed with IPA followed by DI water and dried with a gentle nitrogen stream. The resulting patterned SU-8 film is shown in Figure 3.17. 


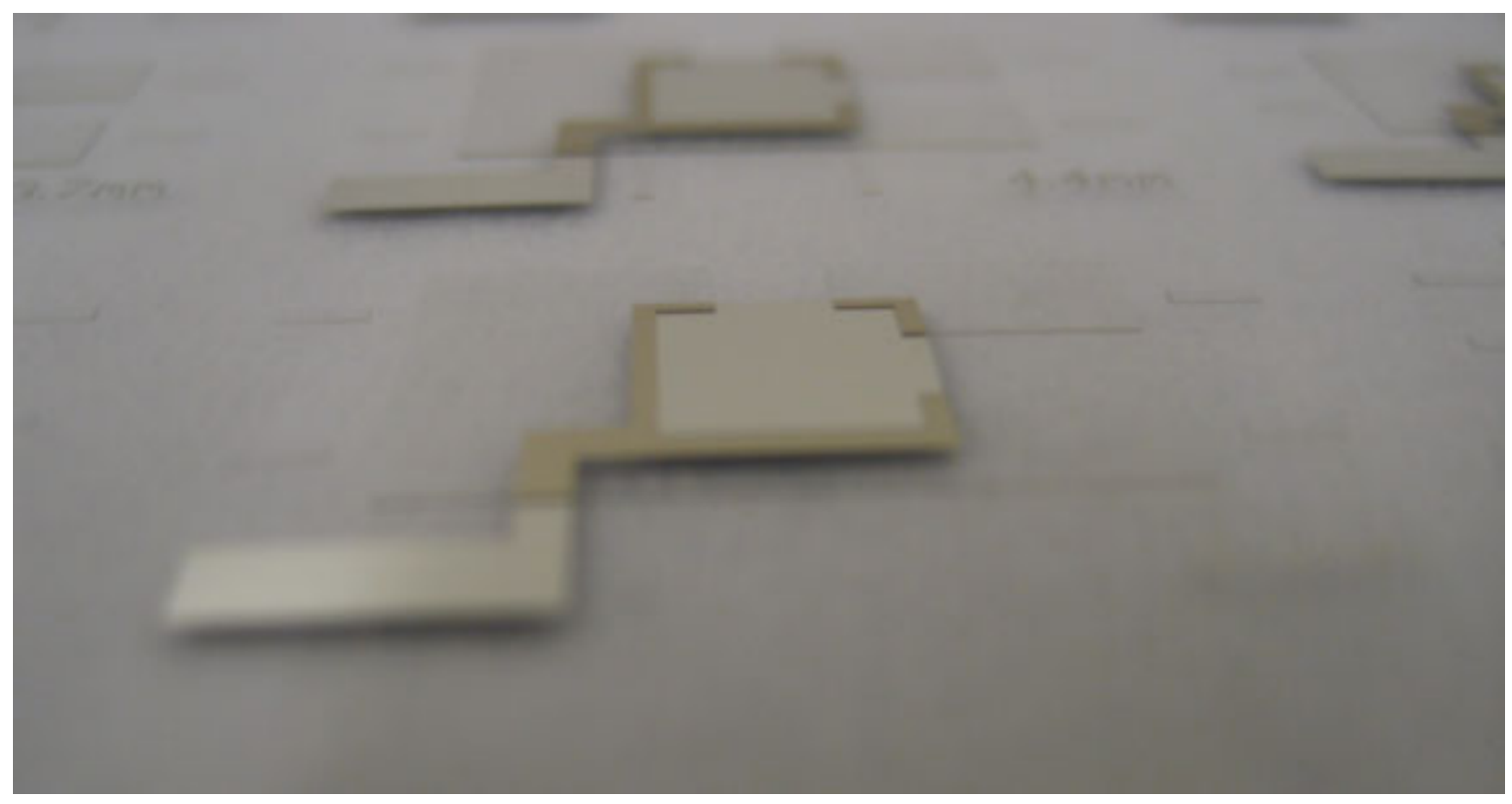

Figure 3.17: The patterned SU-8 gap layer over the aluminum counterelectrode.

\subsection{Final Assembly}

At this point, the individual dies on the silicon wafer were separated by scribing and breaking the wafer along the etched trenches. The silicon dies were placed on the appropriately-sized SU-8 gap feature with the membrane closest to the counterelectrode, and the membranes were aligned by hand to the counterelectrode and SU-8 cavity using the SU-8 alignment features shown in Figure 3.18. The wafers were placed on a hot plate at $150^{\circ} \mathrm{C}$ for 18 minutes while a load of approximately $12.5 \mathrm{KPa}$ was applied to the silicon dies using a beaker of water and a series of glass plates to distribute the force to the individual dies. This baking step completed the cross-linking of the SU-8 and caused the SU-8 to reflow slightly, bonding to the silicon dies and attaching them to the glass wafer. Typically, the SU-8 did not reflow and bond 100\% of the silicon die, but the bond was complete enough to firmly attach the silicon dies to the glass wafer. This incomplete reflow and bonding is shown in Figure 3.19. 

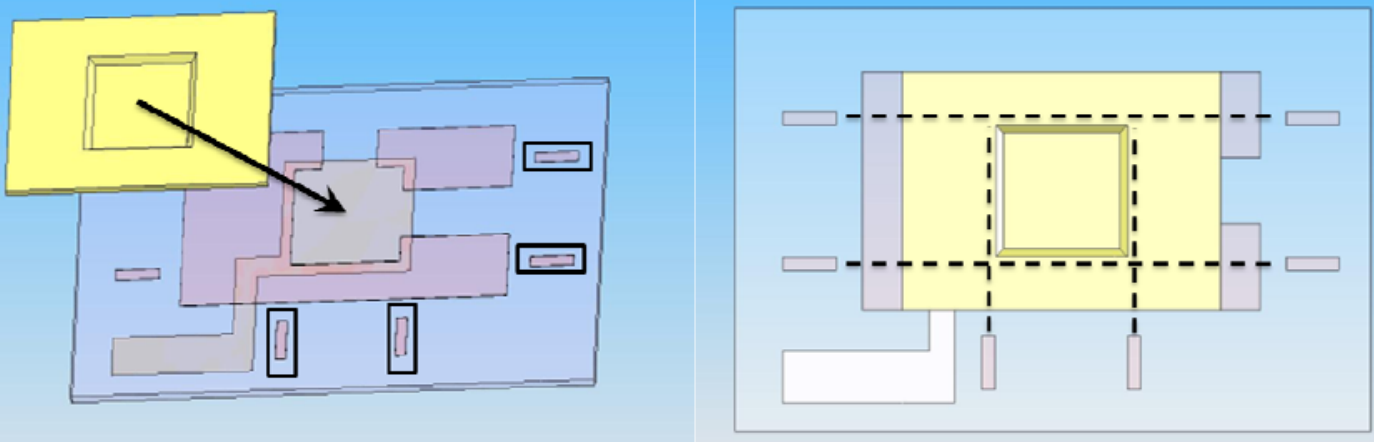

Figure 3.18: Alignment of the membrane to the counterelectrode and gap area.

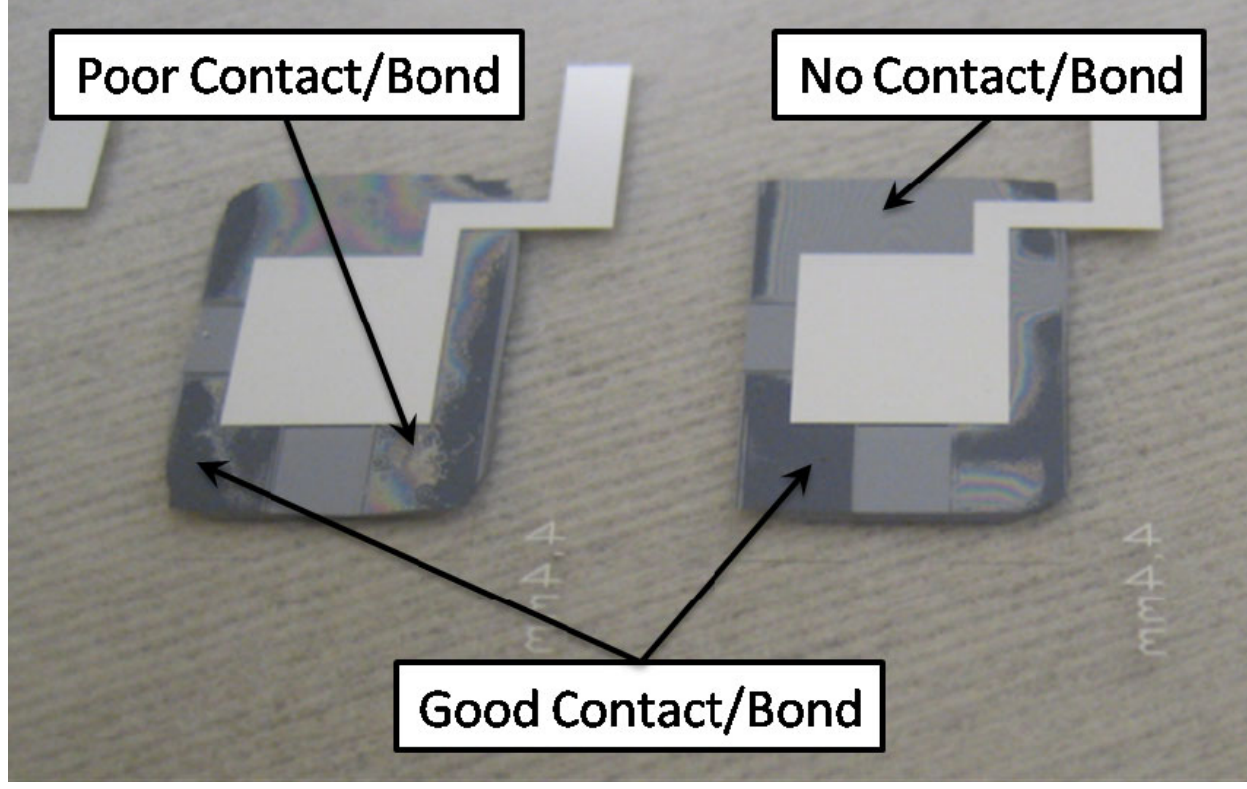

Figure 3.19: Back-side view of the incomplete die bonding.

Figure 3.20 shows the front-side view of a typical device after the bonding process. 


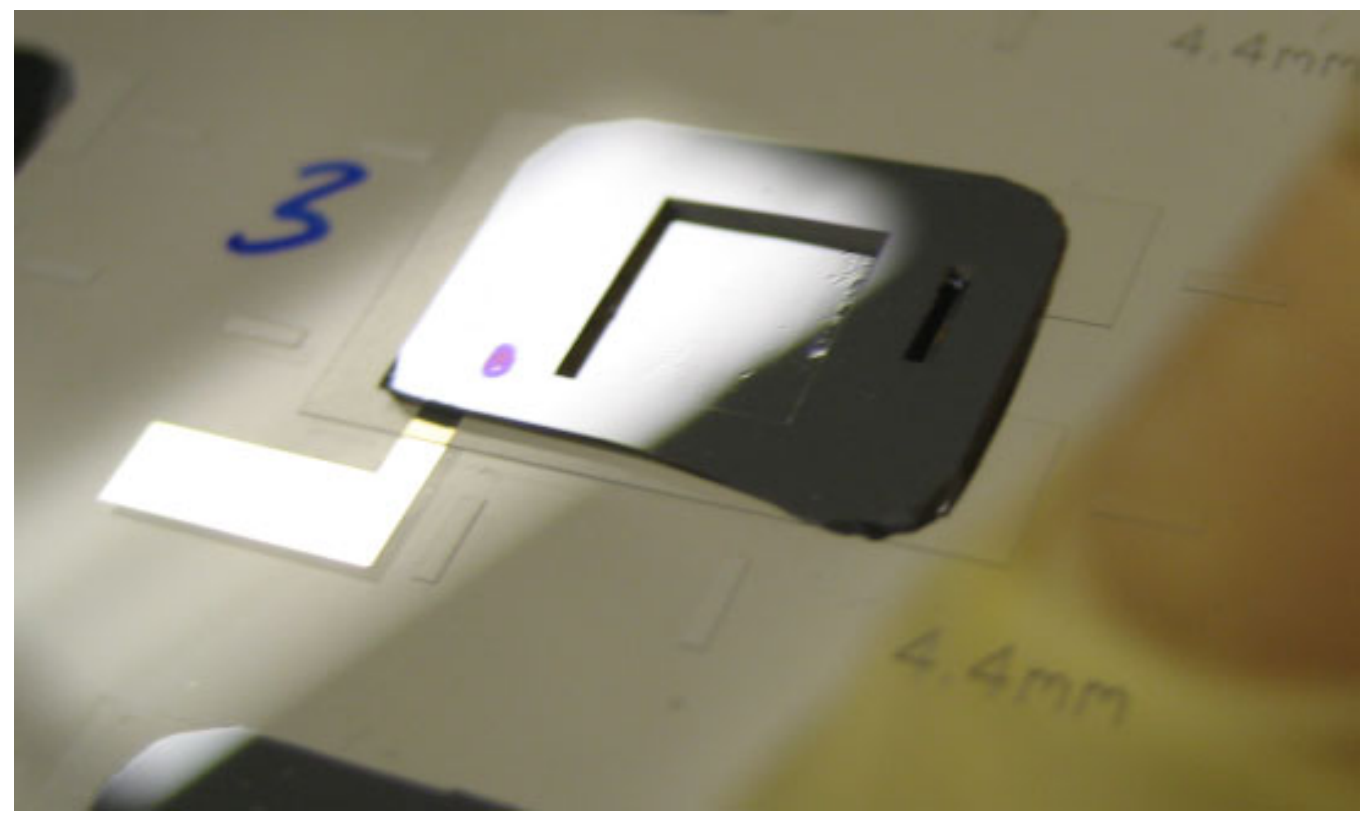

Figure 3.20: A bonded device ready to be separated and characterized.

After bonding, the Pyrex wafers were scribed between the devices using a diamond scribe. The wafer was broken along the scribe lines to separate the individual devices.

\subsection{Surviving Devices}

Table 3.10 lists all the devices that survived the fabrication process and were functional.

\begin{tabular}{|c|c|c|c|}
\hline Device \# & $\begin{array}{c}\text { Membrane Side Length } \\
(\mu \mathrm{m})\end{array}$ & $\begin{array}{c}\text { Membrane } \\
\text { Thickness }(\mu \mathrm{m})\end{array}$ & $\begin{array}{c}\text { Average Gap } \\
\text { Height }(\mu \mathrm{m})\end{array}$ \\
\hline 1 & 4400 & 7 & 54 \\
\hline 2 & 4400 & 7 & 56 \\
\hline 3 & 4400 & 7 & 42 \\
\hline 4 & 4400 & 7 & 43 \\
\hline 5 & 4400 & 7 & 44 \\
\hline 8 & 3700 & 7 & 47 \\
\hline 9 & 3000 & 7 & 46 \\
\hline 10 & 3000 & 7 & 44 \\
\hline
\end{tabular}

Table 3.10: Surviving devices and their key dimensions. 


\subsection{Yield Analysis}

This yield analysis covers only the two wafers that were doped with boron and etched to fabricate the final devices; however, the yield from this batch of wafers is representative of previous attempts. Each wafer was patterned with 50 individual dies for a total of 100 possible devices. As listed in the previous section, the fabrication process resulted in 8 functioning devices, resulting in a final yield of $8 \%$. This yield figure does not include $1300 \mu \mathrm{m}$ and $2200 \mu \mathrm{m}$ silicon membranes that survived etching intact but were not bonded to a counterelectrode; these membranes were not used because they were too small to yield a significant displacement. The main sources of yield loss identified in this fabrication process were the anisotropic wet etching step and the two die separation/dicing steps.

There are several causes of yield loss within the anisotropic wet etching step. First, if there are any pinholes in the oxide on the back side of the wafer, during the etching process these pinholes act as etch windows and can form through-holes in membranes if the pinholes are directly opposite the etch windows. These pinholes are shown in Figure 3.21 and can form during pattern transfer when the photoresist film protecting the backside oxide contains pinholes from the spin-coating and baking processes. In the future, yield losses due to pinholes could be reduced by applying multiple coats of photoresist to the backside of the wafer to better protect the backside oxide during the etch mask pattern transfer step. 


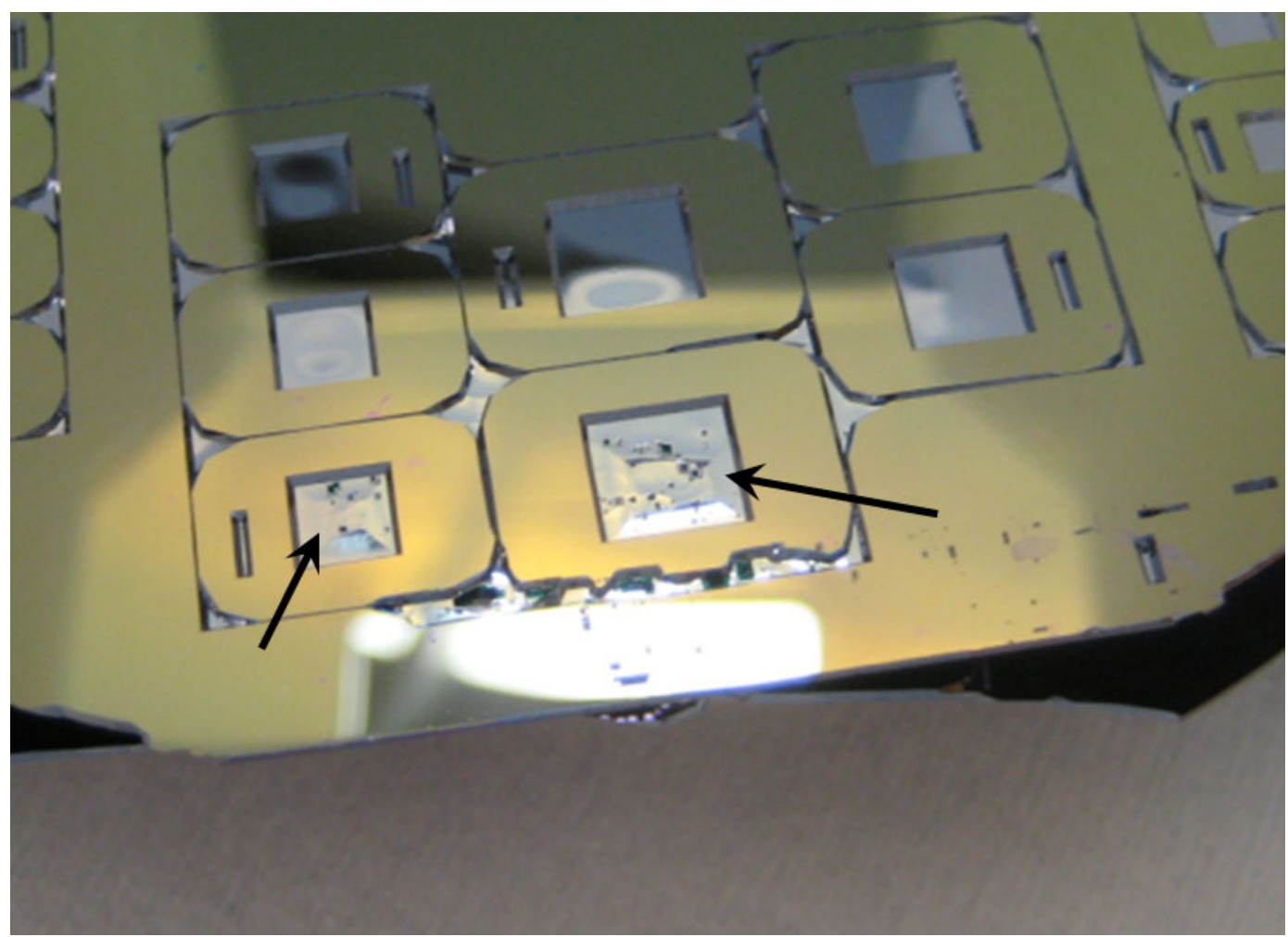

Figure 3.21: Backside oxide pinholes cause etched holes in membranes.

Second, if etchant circulation is not uniform over the surface of the wafer, bubbles can accumulate in the etch pits and cause localized masking. This can manifest itself in two ways: surface tension forces can pin a single bubble in an etch pit causing roughness at the center, or the etchant circulation within the etch pit can be reduced by a physical barrier such as an adjacent wafer or wafer holder. The former is shown in Figure 3.22 and the latter is shown in Figure 3.23. Etchant circulation is particularly troublesome because of the arrangement of the etch vessel and the stirring mechanism. Specifically, if wafers are loaded into the cassette and stacked too close together, etchant circulation between wafers decreases dramatically and significant bubble buildup can occur. To complicate matters, if the etchant is stirred too rapidly the turbulence in the vessel can cause the wafers to bounce around inside the cassette, fracturing wafers and breaking 
membranes inside the etch vessel. Approximately $30 \%$ of the devices did not survive the etching process due to back side pinholes and rough membrane surfaces. Yield losses due to the etching process could be reduced by increasing the spacing between wafers in the cassette or by improving the circulation of the etchant within the vessel. This could be accomplished by introducing an additional stirring mechanism at the top of the vessel in addition to the magnetic stir bar in the bottom of the vessel. Also, IPA could be added to the etchant as discussed earlier.

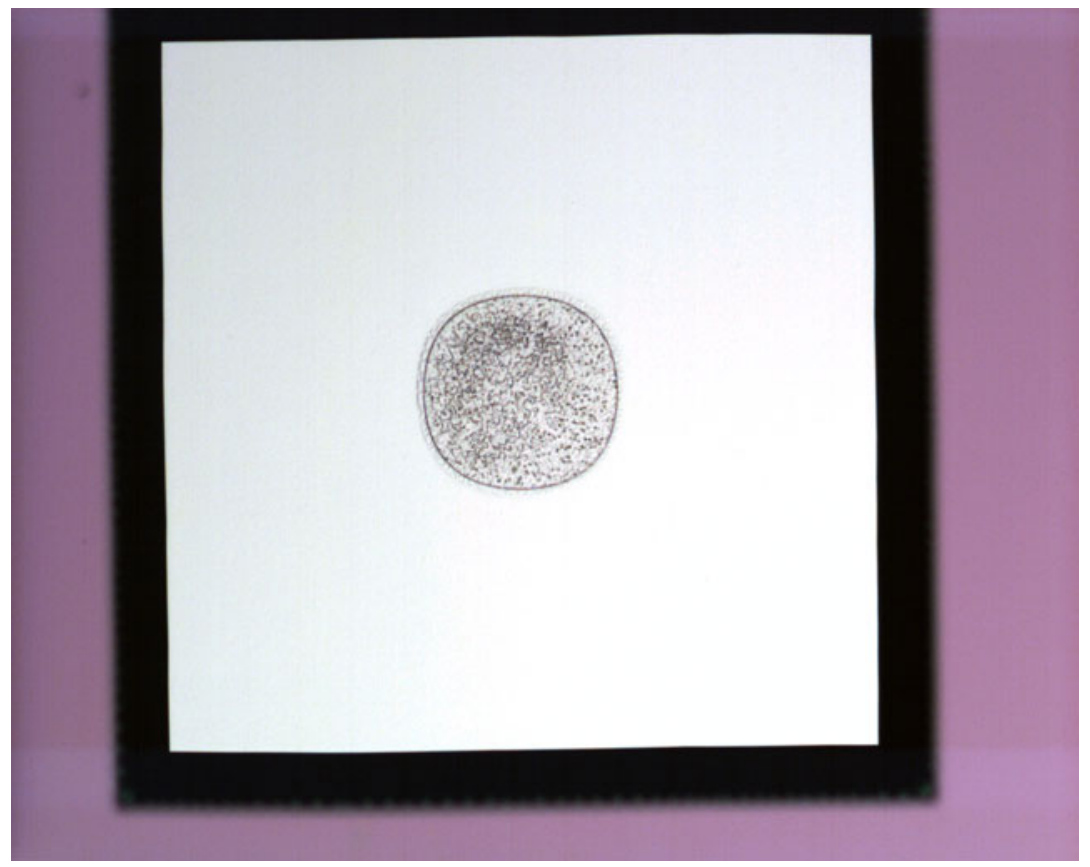

Figure 3.22: Roughness and irregularity on a membrane due to a single bubble in the center of the membrane with a long residence time. 


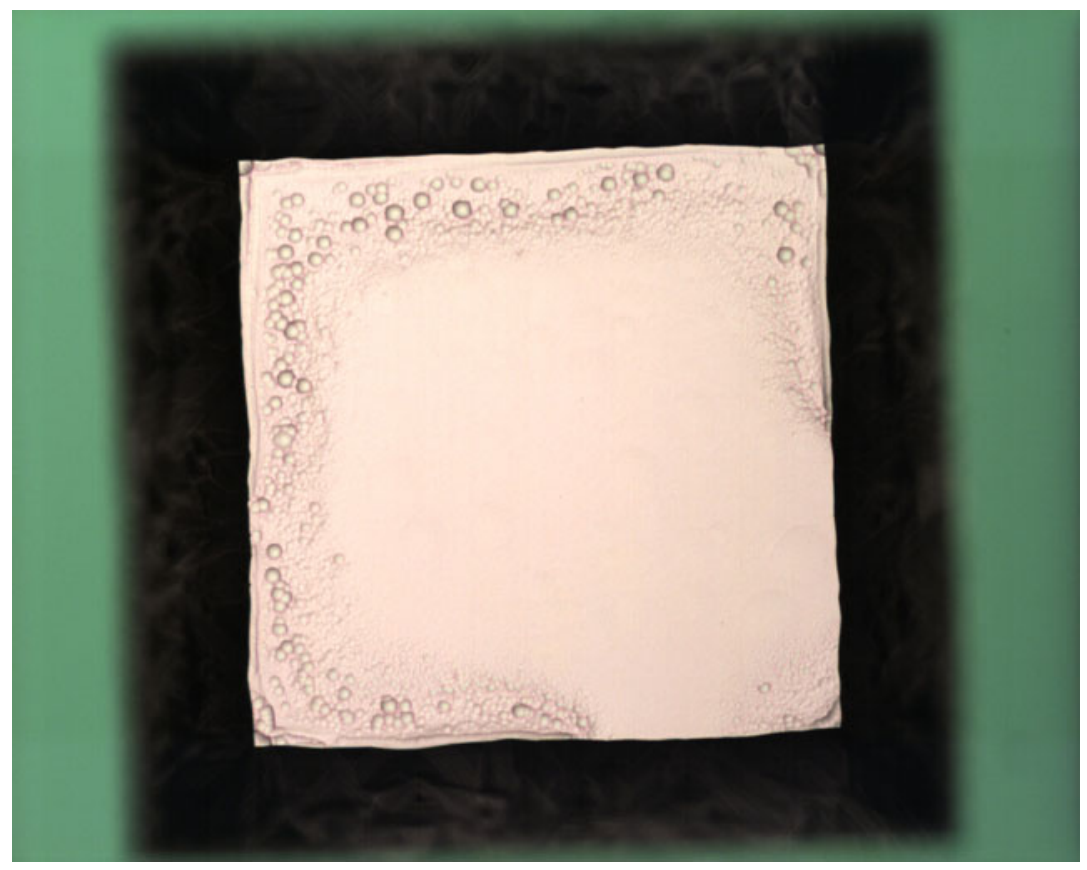

Figure 3.23: Rough membrane edges caused by insufficient etchant circulation from a physical barrier to circulation - in this case, a wafer cassette.

Silicon die separation must be handled carefully because of the fragile nature of the membranes. The silicon etch window lithography mask was designed with trenches to facilitate the separation process; however, a significant amount of force was still required to break the wafer and separate the dies. Die separation was accomplished by scribing along the trench lines with a diamond scribe and then bending the wafer by hand until fracture occurred. The membranes experienced stress when the wafer bent, and some of them fractured along one or more edges or broke completely during the separation process. The die separation process destroyed approximately $15 \%$ of the devices. Die separation losses could be reduced by redesigning the lithography mask with wider trenches which require less bending to fracture. 
Separating the final devices on the Pyrex wafer was even more challenging than separating the silicon dies. The Pyrex wafer was un-etched, and the dicing process relied entirely on scribe lines to control the fracture of the wafer. Pyrex is an amorphous, glassy solid and it does not contain any long-range crystallographic planes along which fracture can propagate; therefore, it is difficult to contain fractures to scribe lines. Several final devices were lost because a crack deviated from the scribe lines and propagated through the device. Some degree of control was achieved over the crack propagation by scribing the same line multiple times, creating a deep scratch. Roughly $10 \%$ of the devices were lost during the separation process due to uncontrolled crack propagation. This scribing process could be improved by using a dedicated glass scribing tool, cutting oil, a straight edge guide, and by applying a light, uniform pressure to the scribe to create a smooth scribe line. 


\section{SECTION 4: MODELING}

\subsection{Summary}

As stated in the design section, a series of equations was developed which were used to design the electrostatically-actuated membrane structures. These equations made certain assumptions about the behavior of the structures, i.e. the initial design equation assumed that the electrostatic force remained constant as the membrane deformed and the nonlinear electrostatic force equation assumed that the membrane remained rigid and deflected in its entirety towards the counterelectrode. Neither of these assumptions are correct; in fact, the membrane deforms towards the counterelectrode while the edges remain fixed and the electrostatic force increases on the parts of the membrane that deform towards the counterelectrode. A more sophisticated modeling technique was sought to predict the equilibrium displacement of the membranes; this modeling predictions will be compared to the design predictions and the actual device performance in a later section of this thesis. Software finite element analysis (FEA) was chosen as this more sophisticated technique, and the commercially available CoventorWare 2008 software package was used to perform the analysis [60].

\subsection{Finite Element Analysis}

CoventorWare is a design, process visualization and FEA software package designed specifically for MEMS devices. Its design tool is process-based; this means that instead of drawing a 3-D model, the user inputs the microfabrication process layer-by-layer and defines the masks for each lithography step. This approach is more intuitive for users 
with a microfabrication background. The general CoventorWare process used to generate a 3-D model of a membrane structure is shown in Table 4.1.

\begin{tabular}{|c|c|c|c|c|}
\hline Step & Action & Material & Thickness & Mask \\
\hline 1 & Substrate & $(100)$ Silicon & $50 \mu \mathrm{m}$ & Substrate \\
\hline 2 & Deposit & Aluminum & $200 \mathrm{~nm}$ & Substrate \\
\hline 3 & Deposit & $\mathrm{SiO}_{2}$ & $44 \mu \mathrm{m}$ & Substrate \\
\hline 4 & Deposit & $(100)$ Silicon & $7 \mu \mathrm{m}$ & Substrate \\
\hline 5 & Remove & $\mathrm{SiO}_{2}$ & N/A & N/A \\
\hline
\end{tabular}

Table 4.1: Generic CoventorWare process to model membranes.

In this particular process a substrate of arbitrary thickness, defined by the "Substrate" mask, was coated with a $200 \mathrm{~nm}$-thick film with the electrical properties of aluminum forming the counterelectrode. Then, $44 \mu \mathrm{m}$ of an arbitrary sacrificial material was deposited on top of the aluminum; this layer set the thickness of the electrode gap and was removed later. Next, a $7 \mu \mathrm{m}$-thick layer with the mechanical properties of singlecrystal (100) silicon was deposited on the oxide to form the membrane, as shown in Figure 4.1. 


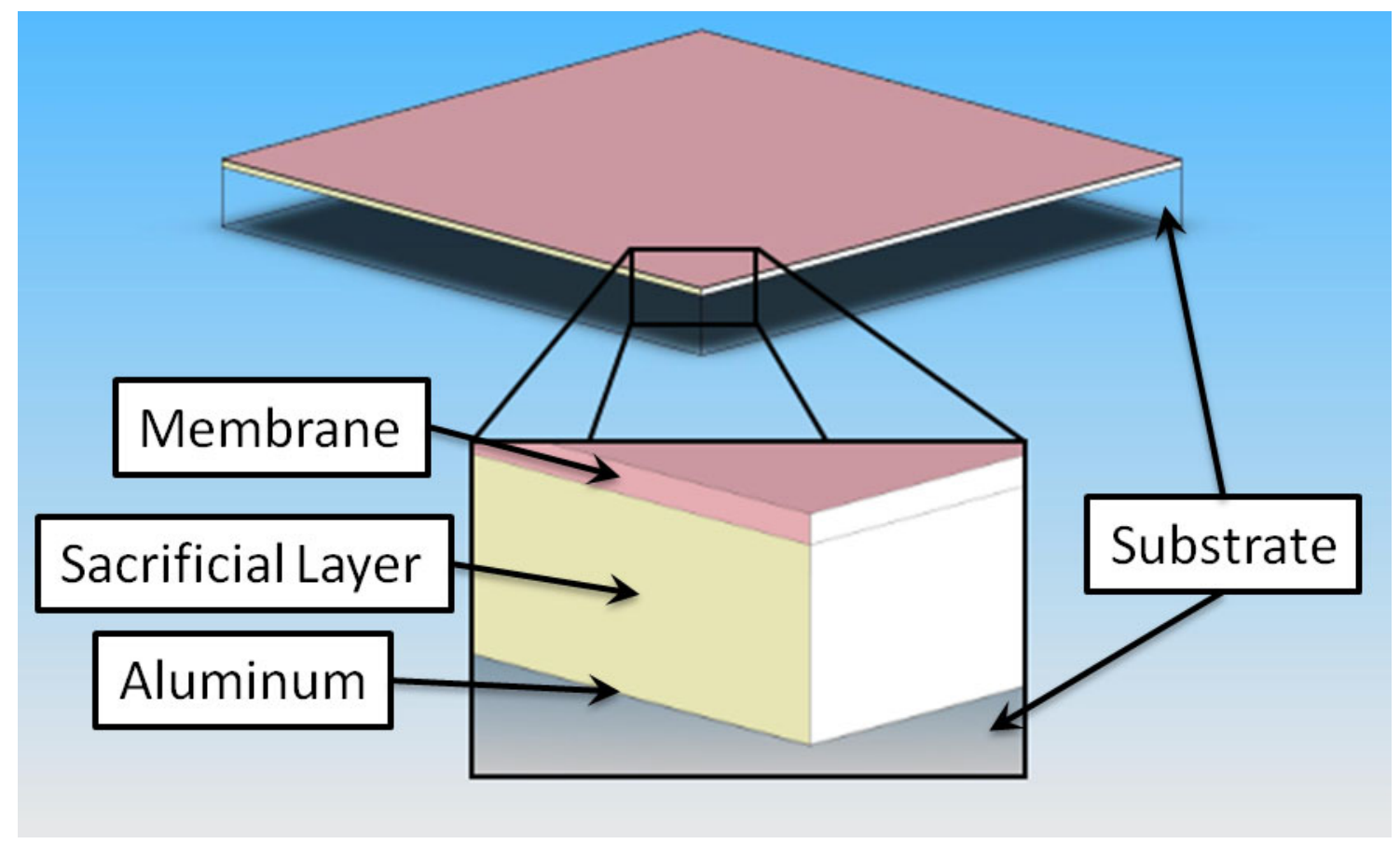

Figure 4.1: The "stack" of layers used to create the Coventor model.

Finally, the sacrificial silicon dioxide was removed, creating a cavity into which the suspended membrane could deflect. The substrate layer was ignored for the FEA because it influenced neither the mechanical nor the electrical properties of the device. Each of the deposited layers was defined by the same "Substrate" mask, yielding a model which was essentially a stack of square layers. When finished, the model contained only the deforming membrane and the counterelectrode layers and the other layers were ignored, as shown in Figure 4.2. 


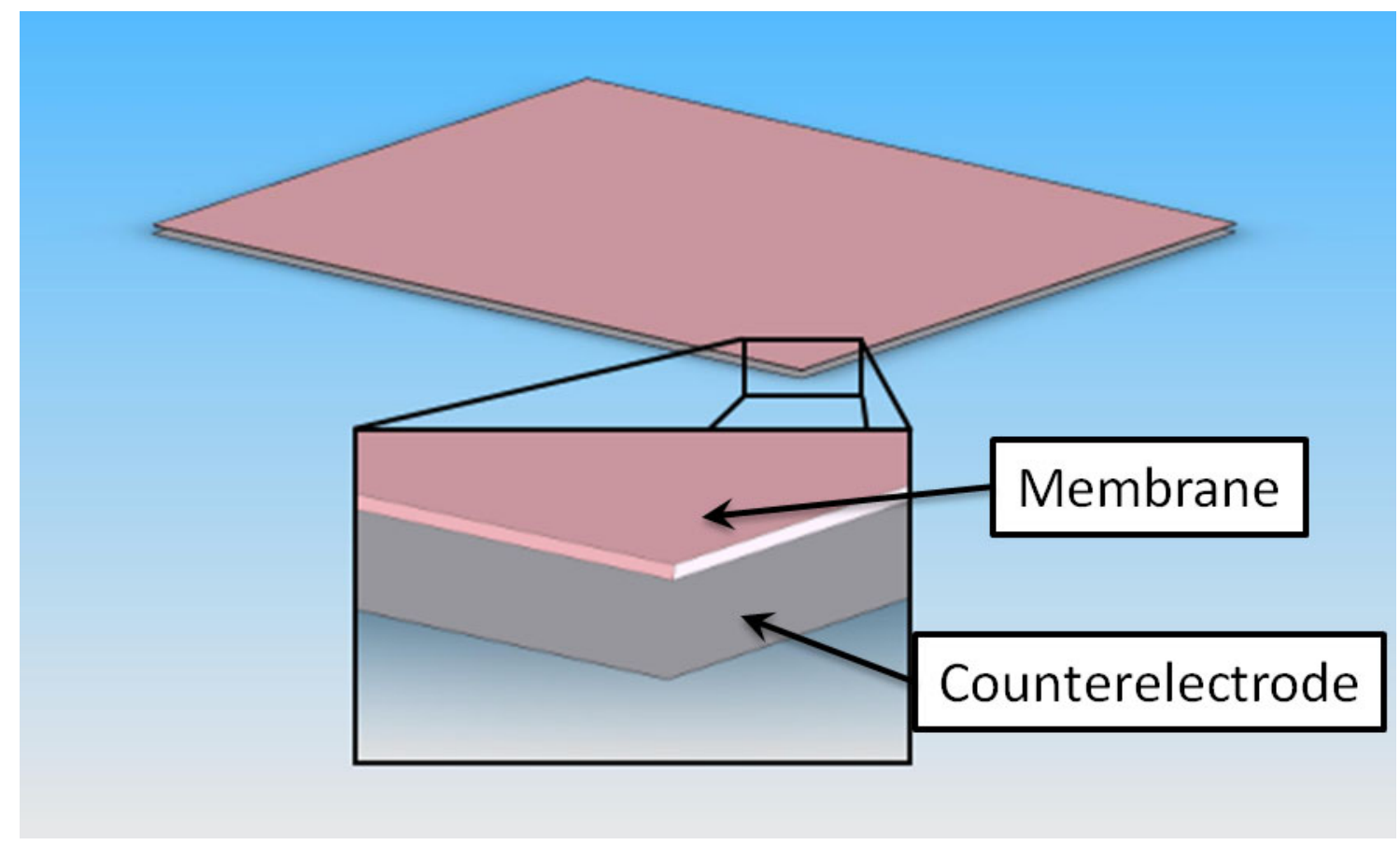

Figure 4.2: The FEA model containing only the counterelectrode and membrane.

Once the process was defined, a 3-D model was generated from the process which was used to define locations for restraints and loads and to define the mesh. Various mesh element options were available to the user, including tetrahedral and brick elements. 27node quadratic bricks with one element through the membrane thickness were used in this study because the membrane itself was a rectangular hexahedron and the mesh could be fully populated by a whole number of these hexahedral elements. Tetrahedral elements could be used when generating a mesh for a model that contains non-rectilinear geometry, such as components with crystallographic facets or non- $90^{\circ}$ angles.

Aside from the unique process-based design system and the control over the mesh elements, a significant advantage of CoventorWare over other software packages is that it includes solvers for electrostatic and coupled electrostatic-mechanical analysis. The 
coupled electrostatic-mechanical solver, CosolveEM, combines the mechanical and electrostatic solvers and allows the user to define mechanical restraints on the device and apply voltage biases between parts; it then runs an iterative process to determine the equilibrium displacement of each part. The CosolveEM solver first generates capacitance information for each mesh element, then applies the voltage bias to determine the electrostatic force on each element. This force information is fed into the mechanical solver which computes the displacement of each element. The electrostatic solver then computes new capacitance information for the elements, determines the charge and force on each element and feeds this new force information to the mechanical solver which recomputes the displacement. This iterative process continues until the change in displacement between the two previous iterations drops below some threshold tolerance, indicating that the analysis has converged. The software displays selected results, such as maximum displacement, in a table; alternatively, a 3-D model of the device showing the results can be viewed. Figure 4.3 below shows a typical 3-D model showing the displacement of the device. 


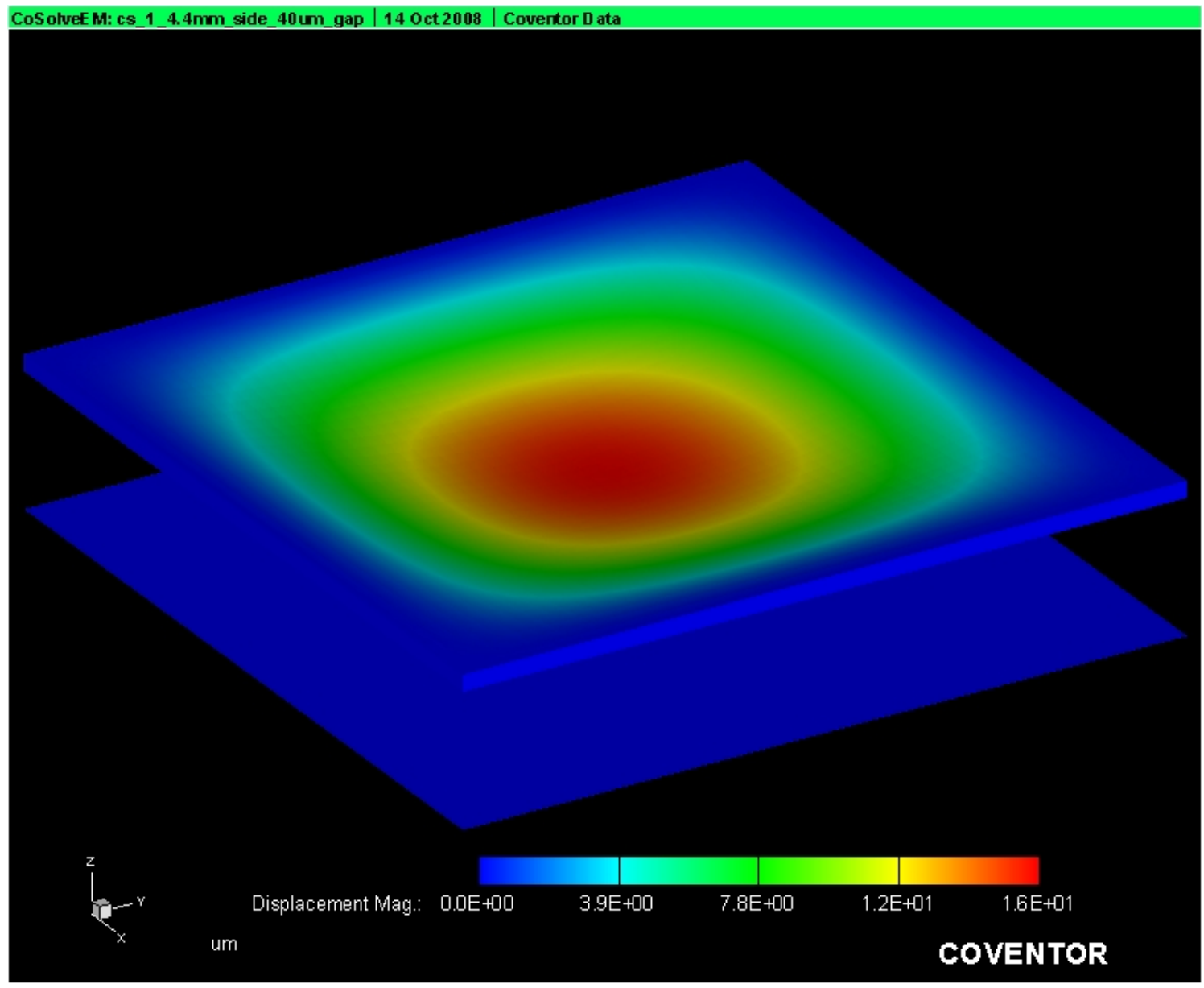

Figure 4.3: Typical output from Coventor FEA showing displacement.

As expected, the maximum displacement is found in the center of the membrane. Figure 4.4 below shows a typical model of the Von Mises stress in the membrane due to its deformation. The maximum stress is found in the center of each membrane edge, which agrees with the equation for stress in a membrane, Eq. (2.3). 


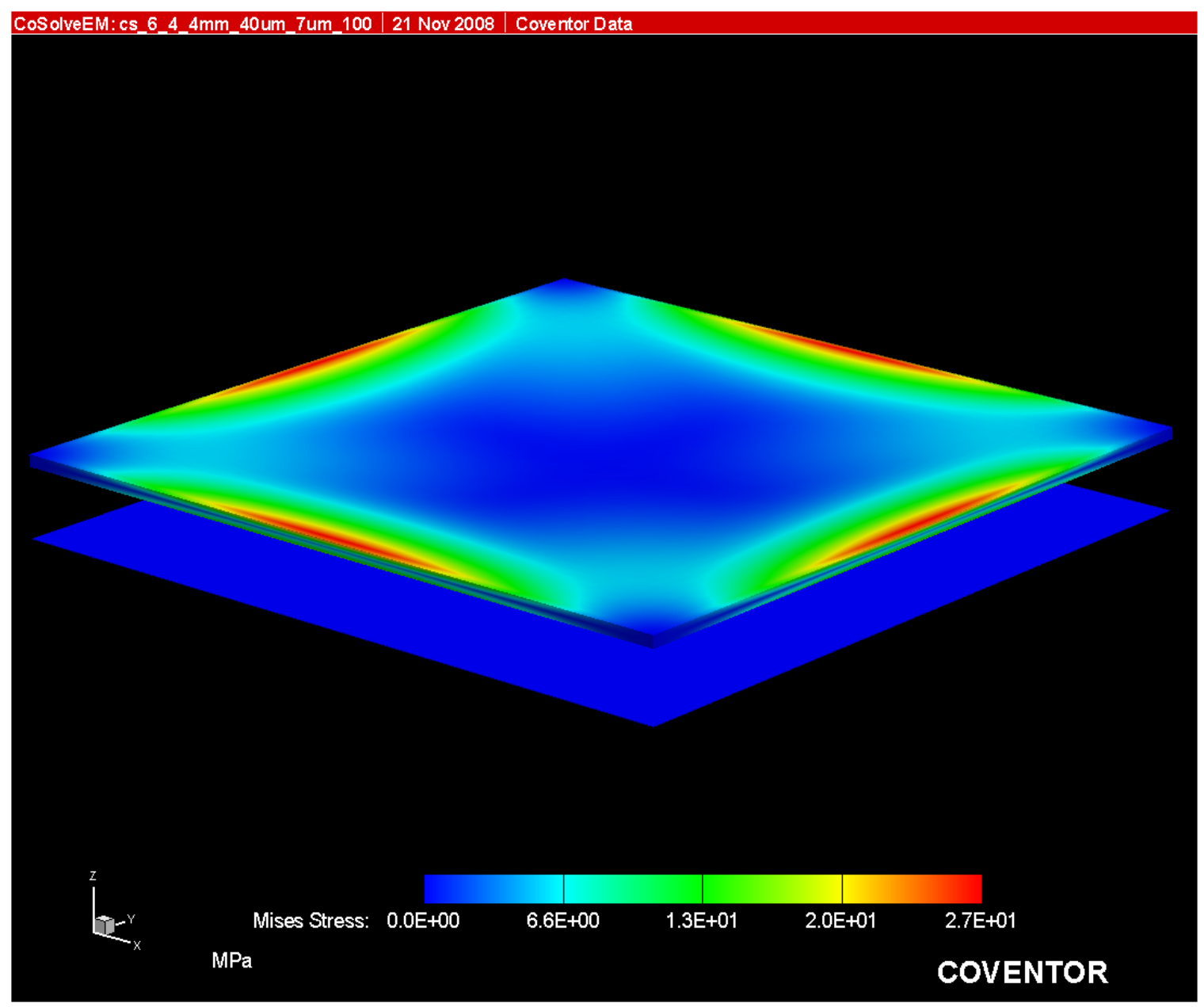

Figure 4.4: Von Mises stress in a membrane under actuation.

Figure 4.5 below shows the charge distribution on the surface of the membrane resulting from electrostatic actuation. As the membrane deforms towards the counterelectrode, the capacitance in the center of the membrane increases because of the decreasing gap. This increased capacitance causes more charge buildup due to the voltage bias, increasing the electrostatic force on the center of the membrane. 


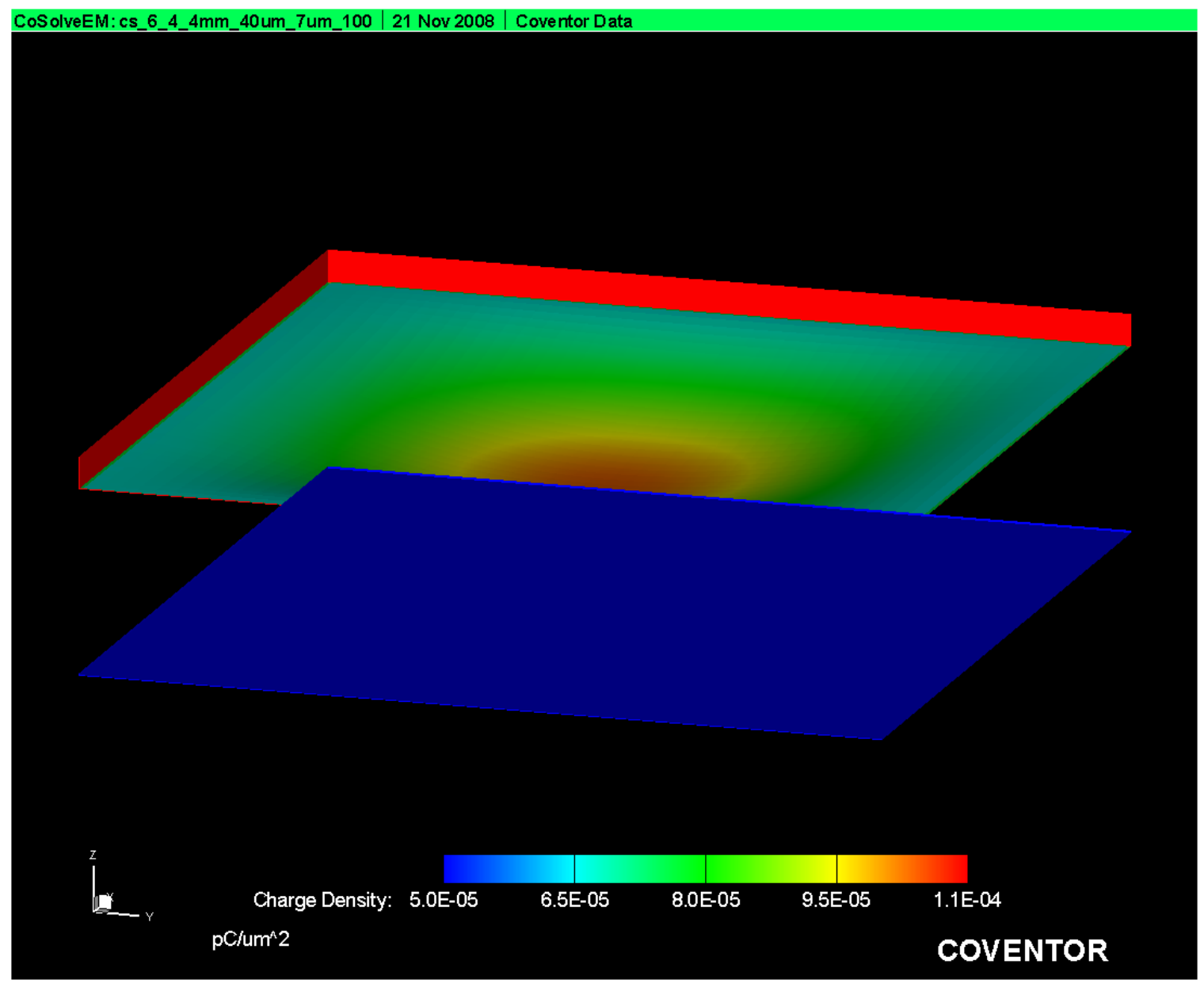

Figure 4.5: Charge distribution on the membrane under actuation.

FEA was performed after the devices were fabricated so that the actual device dimensions could be used to generate the models, rather than the design dimensions. In addition to modeling the fabricated devices, a study was conducted to investigate how device dimensions affected equilibrium displacement. Specifically, the maximum displacement at different actuation voltages was modeled as a function of membrane thickness, side length and electrode gap thickness. This study was done to understand how various changes in the processing and/or device dimensions would affect the maximum displacement of the membrane. This was accomplished by creating a base 
design and modifying this design to investigate the effects of varying dimensions. This base design was built around the specifications listed in Table 4.2, which were chosen to most closely match the fabricated device dimensions.

\begin{tabular}{|c|c|}
\hline Side Length $(\mu \mathrm{m}):$ & 4400 \\
\hline Membrane Thickness $(\mu \mathrm{m}):$ & 7 \\
\hline Electrode Gap $(\mu \mathrm{m}):$ & 44 \\
\hline
\end{tabular}

Table 4.2: Base design dimensions for FEA.

Before any experiments were performed, the optimal mesh size was determined. This was determined by taking the base device design presented in the table above, actuating it with a $200 \mathrm{~V}$ bias and refining the mesh element size until the equilibrium displacement converged. As the mesh elements become smaller and more numerous, the accuracy of the analysis typically increases; however, increasing the number of elements makes the analysis more computationally intensive and time-consuming. Thus, there is some optimal balance between accuracy and the time required to complete the analysis. The results of this mesh size study are shown in Figure 4.6. 


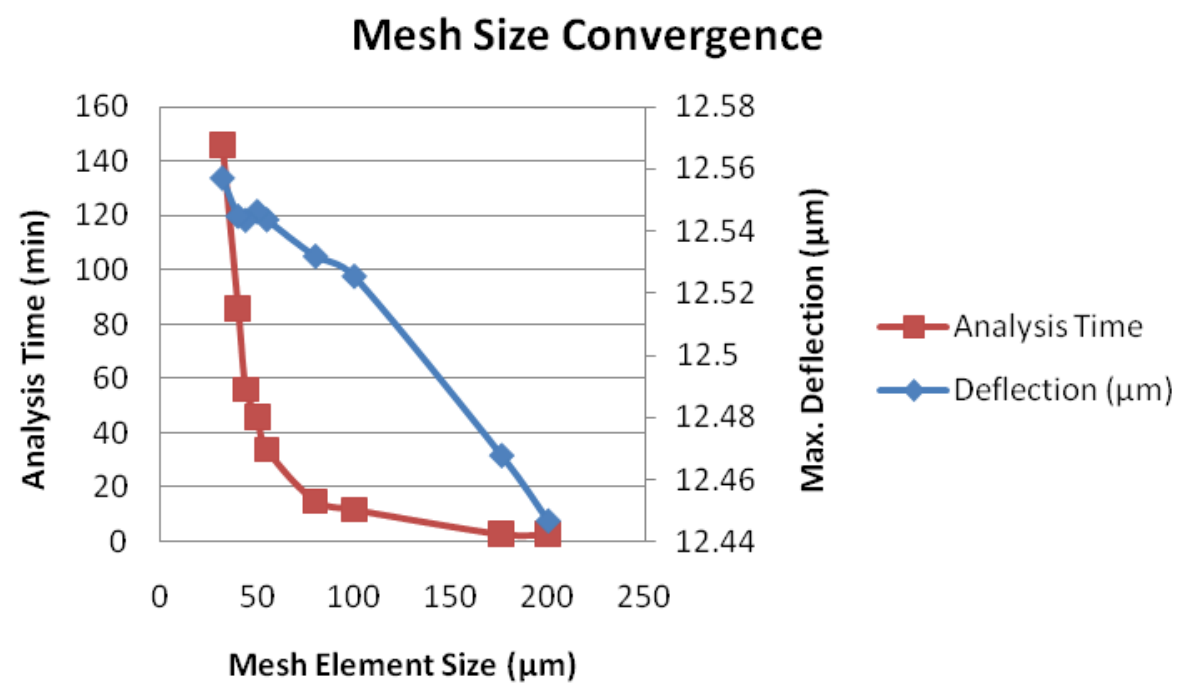

Figure 4.6: Mesh size convergence study.

As this figure shows, the analysis time rapidly increases for mesh elements smaller than approximately $100 \mu \mathrm{m}$, while the accuracy of the analysis increases only slightly. Therefore, $100 \mu \mathrm{m}$ was chosen as the optimal mesh element size.

\subsection{FEA Results}

Table 4.3 lists the different experiments that were performed to investigate the effects of device dimensions on displacement.

\begin{tabular}{|c|c|c|c|c|}
\hline Experiment & $\begin{array}{l}\text { Side Length } \\
(\mu \mathrm{m})\end{array}$ & $\begin{array}{c}\text { Membrane } \\
\text { Thickness }(\mu \mathrm{m})\end{array}$ & $\begin{array}{l}\text { Electrode Gap } \\
(\mu \mathrm{m})\end{array}$ & $\begin{array}{c}\text { Actuation Voltage } \\
\text { (V) }\end{array}$ \\
\hline 1 & $\begin{array}{c}3000,3700 \\
4400\end{array}$ & 7 & 44 & $\begin{array}{c}50,100,150,200, \\
250,300 \\
\end{array}$ \\
\hline 2 & 4400 & 7 & $\begin{array}{c}36,40,44,48, \\
52,54\end{array}$ & $\begin{array}{c}50,100,150,200, \\
250,300 \\
\end{array}$ \\
\hline 3 & 4400 & $5,6,7,8,9$ & 44 & $\begin{array}{c}50,100,150,200 \\
250,300\end{array}$ \\
\hline
\end{tabular}

Table 4.3: FEA investigation of device dimensions. 
To investigate the effects of these device dimensions, 3-D models were generated for each combination of dimensions. For example, in Experiment 1, three different models were generated, each with a $7 \mu \mathrm{m}$-thick membrane and a $44 \mu \mathrm{m}$ electrode gap. One model had a membrane side length of $3000 \mu \mathrm{m}$, another had a side length of $3700 \mu \mathrm{m}$ and the third model had a side length of $4400 \mu \mathrm{m}$, as shown in the table above. Each of these models was actuated with the series of voltages shown in the right-hand column of the table above, and the maximum displacement at each voltage was recorded. The convergence tolerance was set at 0.001 , meaning that when the difference in displacement between the last two iterations of the solver dropped below $0.1 \%$, the analysis had converged. The results of these experiments are shown in the following figures.

Experiment 1: Deflection vs. Side Length

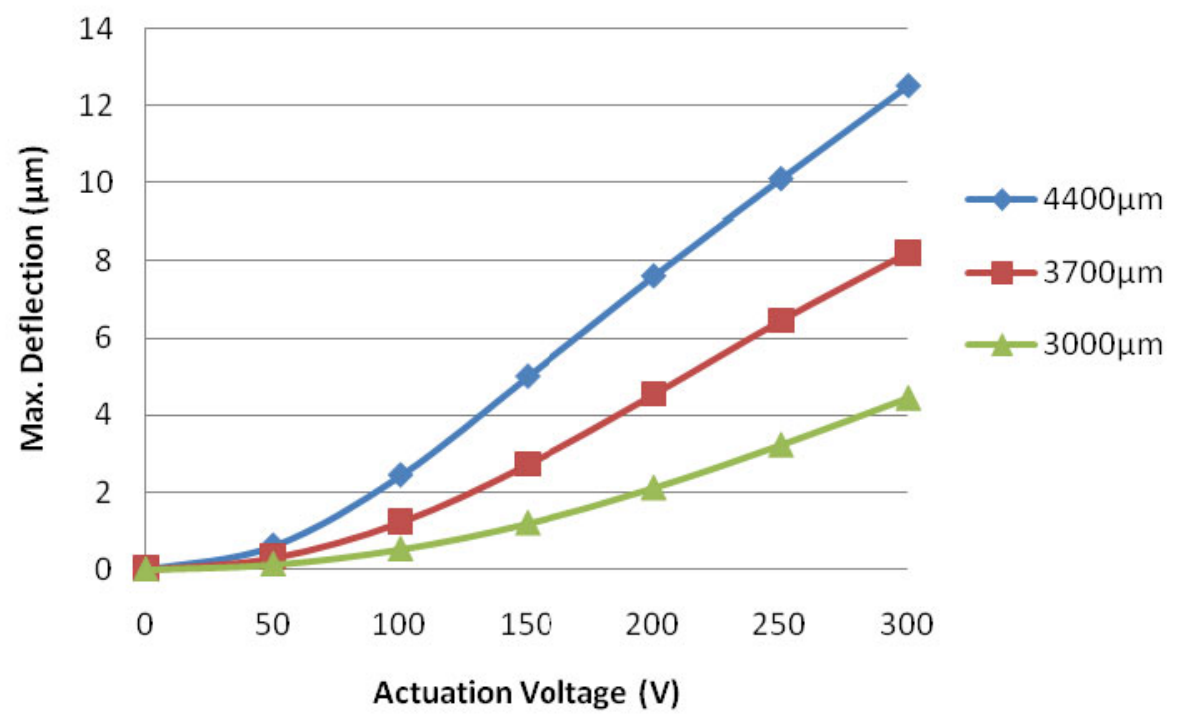

Figure 4.7: Results of the deflection vs. side length experiment. 
Experiment 1 shows the effect of changing the membrane side length. As expected, a smaller membrane deflects significantly less than a larger membrane due to its greater flexural stiffness.

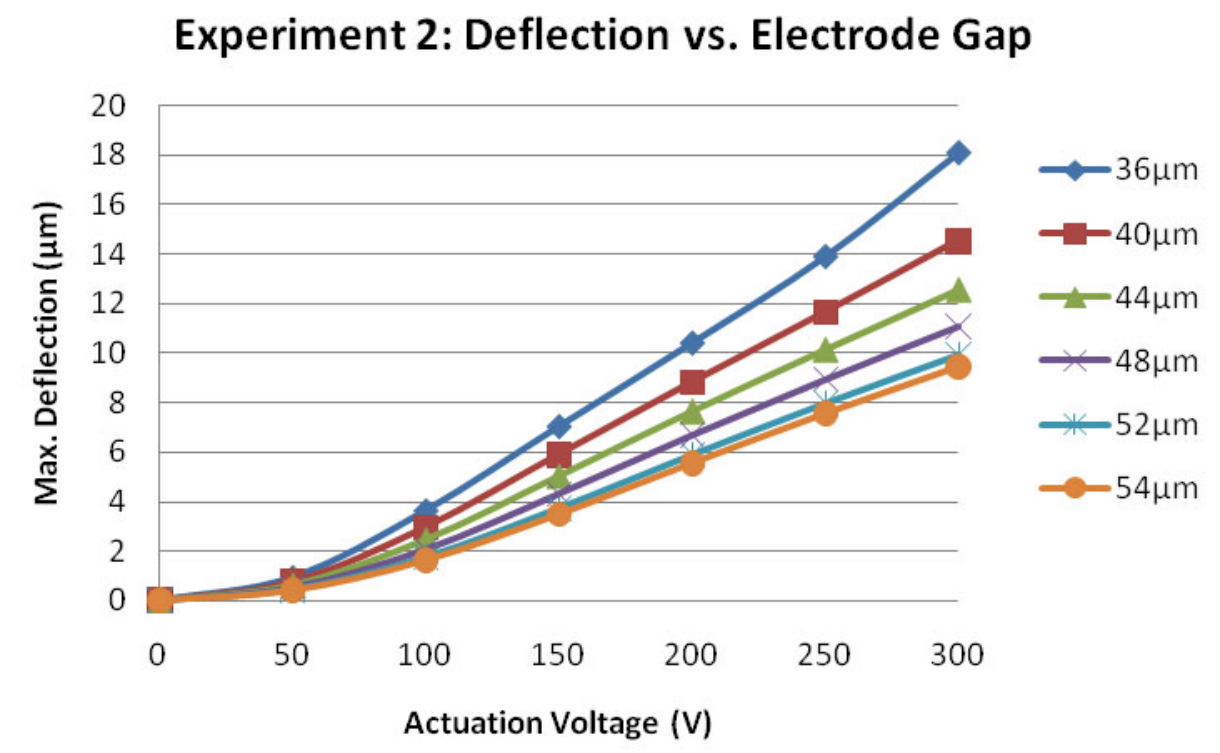

Figure 4.8: Results of the deflection vs. electrode gap experiment.

Experiment 2 shows how the electrode gap affects the maximum deflection of the membrane. It is interesting to note that the predicted deflection increases much more linearly with voltage for larger gaps. This is intuitive - for smaller gaps, not only is the initial electric field greater, producing a greater attractive force and greater displacement, but the deflection is a much greater fraction of the gap producing a greater change in the electric field. 


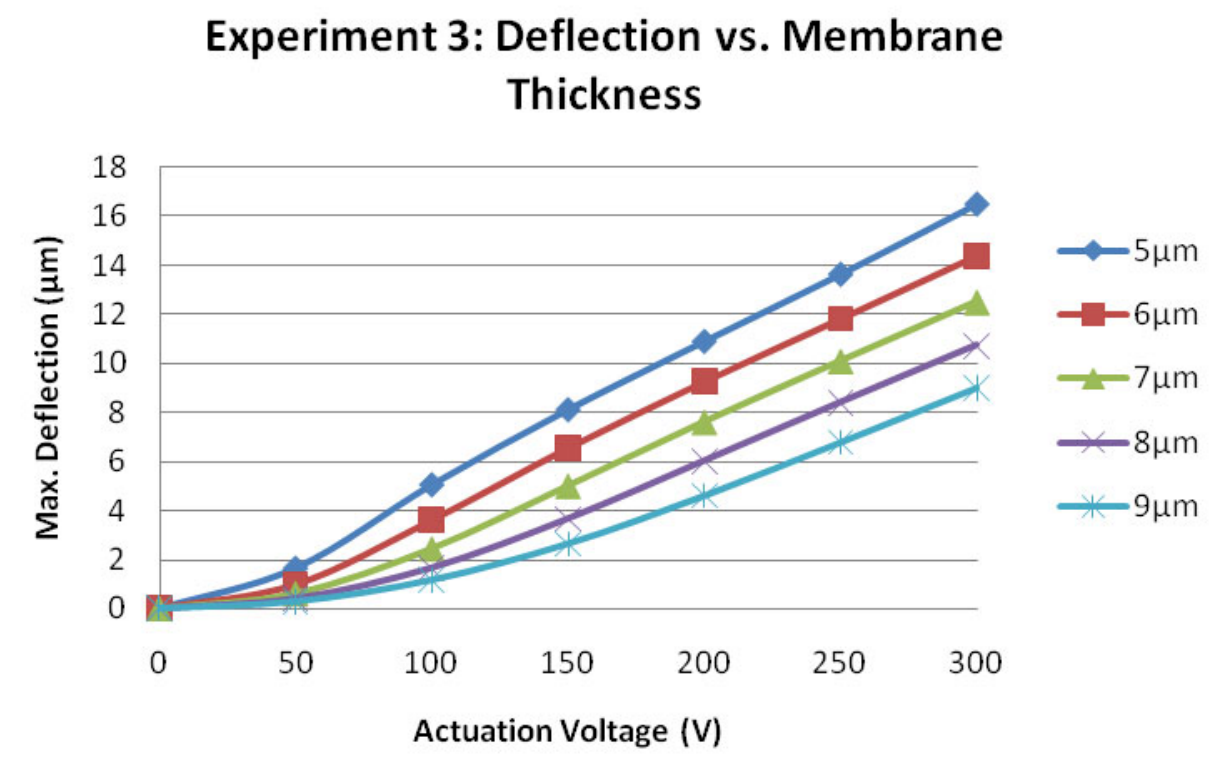

Figure 4.9: Results of the deflection vs. membrane thickness experiment.

The results of Experiment 3 predict how deflection will respond to changes in membrane thickness. Interestingly, the curves appear to become parallel, suggesting that there is some region in which the membrane thickness only changes the deflection by a scalar offset. In general, all of these experiments predict that deflection increases fairly linearly with actuation voltage once the membrane deflects more than a few microns. 


\section{SECTION 5: DEVICE CHARACTERIZATION}

\subsection{Summary}

This section will discuss the electrostatic actuation and characterization of the membrane devices. The main focus of the characterization was to determine the maximum deflection occurring at the center of the membrane under actuation with a series of voltages. The membranes were actuated with a static DC bias inside the stylus profilometer which was then used to scan the profile of the membrane under actuation. This profiling was performed for a series of actuation voltages and care was taken to ensure that the scan passed through the center of the membrane in order to capture the maximum deflection. The profiles of the membrane under actuation were then compared to the profile of the relaxed membrane to determine the deflection due to actuation.

\subsection{Actuation Setup}

The actuation setup, shown in Figure 5.1, consisted of an AdvancedMEMS 2-channel high-voltage (HV) MEMS driver connected to a laptop running a customized LabView graphical user interface (GUI). The driver output was connected to the device using a coaxial BNC cable terminated with alligator-style clips, 22-gauge lead-wire and 3M 1181 conductive copper tape, shown in Figure 5.2. The copper tape was used to make the final connection to the counterelectrode contact pad and the silicon die. Soldering was attempted to create a more robust connection between the lead-wires and the two device components, however, neither surface was wetted by the solder. 


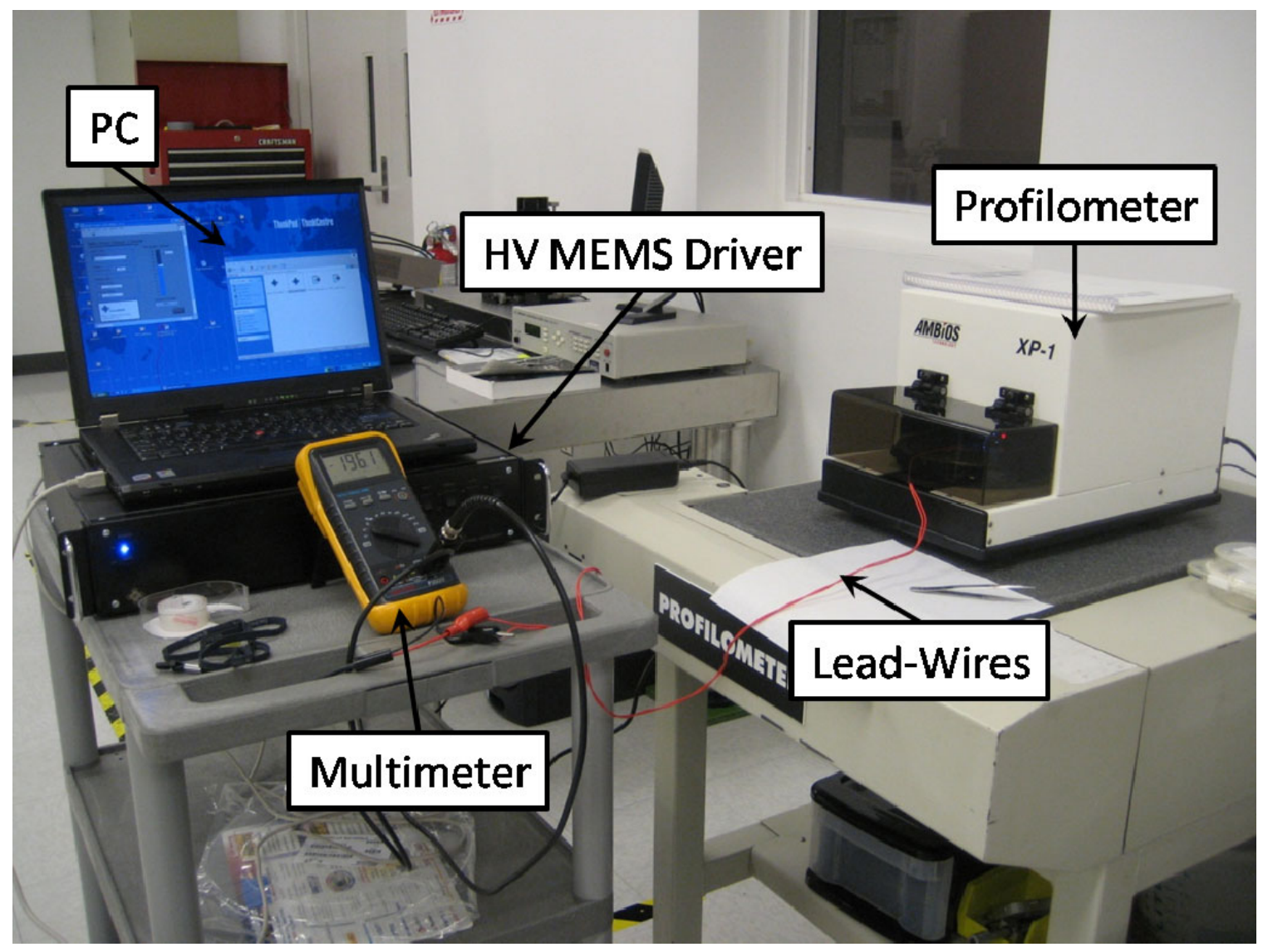

Figure 5.1: The actuation and characterization setup.

The device under testing (DUT) was placed on the stage of the profilometer and $3 \mathrm{M}$ Scotch tape was used to secure the glass slide and lead-wires to the stage, as shown in Figure 5.3. 


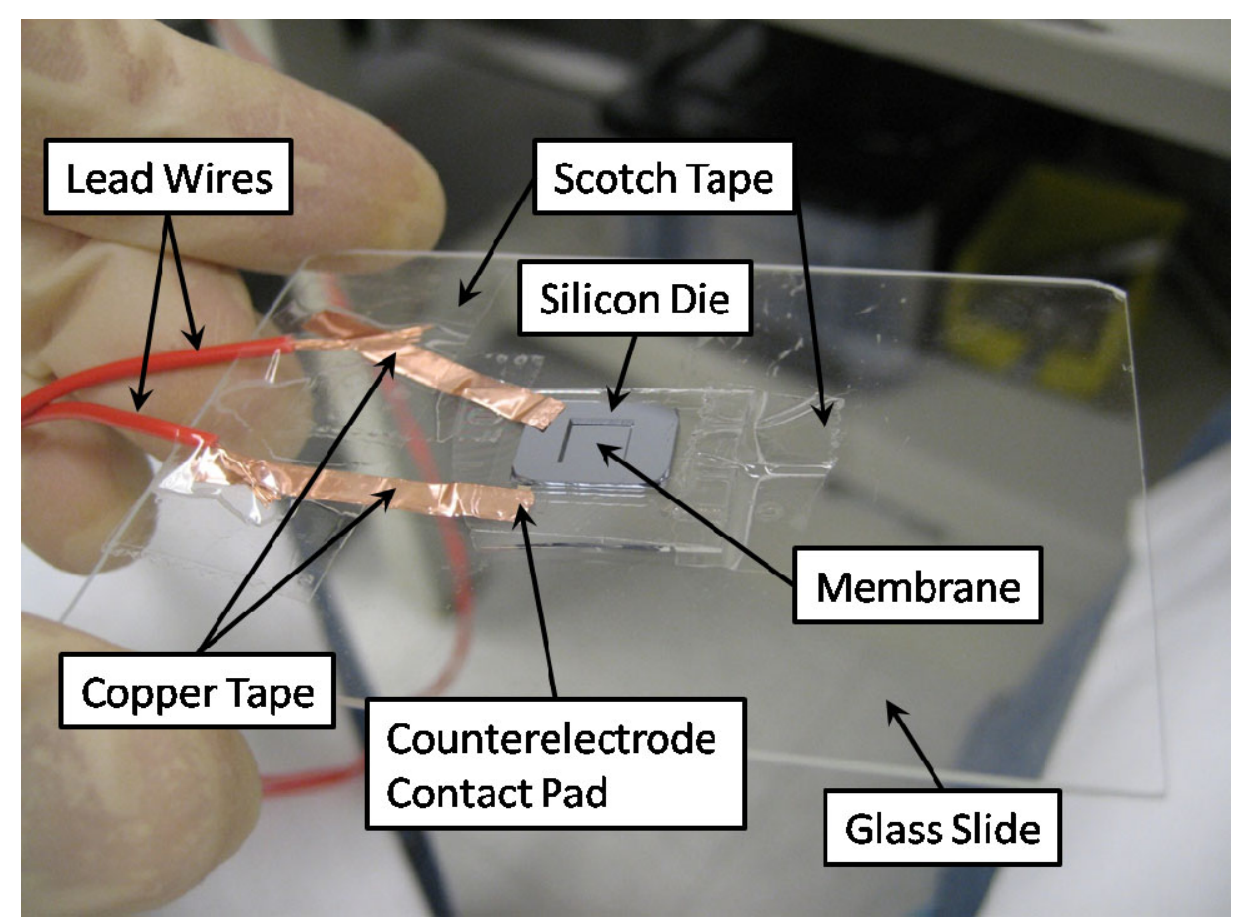

Figure 5.2: The device with attached lead-wires.

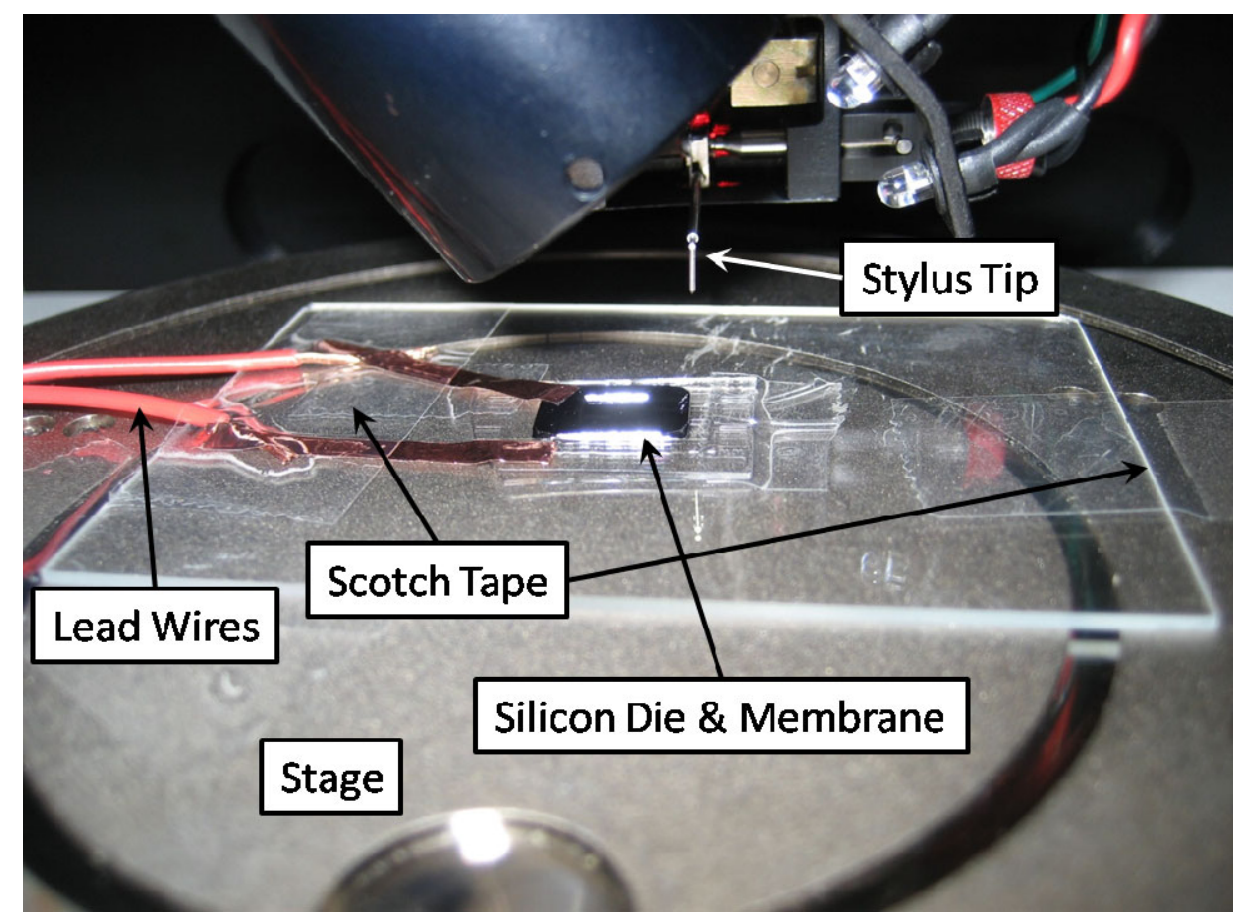

Figure 5.3: The DUT attached to the profilometer stage. 
A customized GUI provided by AdvancedMEMS was used to generate a static DC voltage bias with the HV driver and to actuate the devices. The profilometer was used to scan the surface of the membrane while holding the actuation voltage constant, and the stylus force was reduced to $1.0 \mathrm{mg}$ to minimize any deflection due to the stylus. Each device was actuated and scanned at the following voltages: $0 \mathrm{~V}$ (off), $50 \mathrm{~V}, 100 \mathrm{~V}, 150 \mathrm{~V}$, $200 \mathrm{~V}, 250 \mathrm{~V}$ and $300 \mathrm{~V}$. The $0 \mathrm{~V}$ (off) scan was used to normalize the membrane topology and stylus force for subsequent scans. Several devices were characterized multiple times to determine the repeatability of the actuation, and one device was tested after being actuated approximately $10^{7}$ times by an AC actuation voltage to determine if the devices exhibited any fatigue.

\subsection{Interpreting the Results}

After scanning a device at each of the actuation voltages prescribed in this section, the profilometer scans were compiled and superimposed so that a qualitative estimate of the deflection could be obtained. Figure 5.4 shows a typical set of superimposed scans. As the actuating voltage was increased, the membrane deflected further towards the counterelectrode, causing the profile to shift from relaxed (black scan) to fully actuated (blue). This figure also shows the cratering that was evident on some of the devices as well as the bowing in the membrane in the absence of an actuating voltage. 


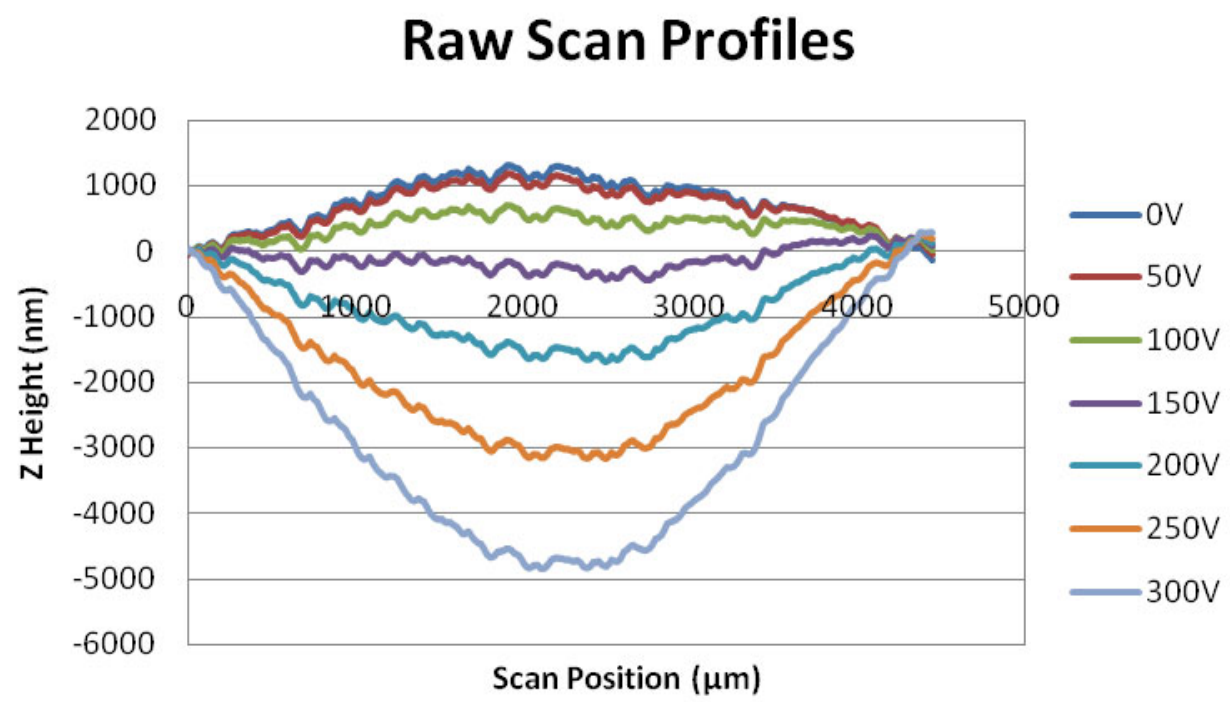

Figure 5.4: Superimposed profilometer scans of a typical membrane under actuation.

After the device was scanned at each actuating voltage, the scan data was imported into a spreadsheet and each actuated scan was normalized to the $0 \mathrm{~V}$ scan. This was done by subtracting the vertical displacement at each point of the $0 \mathrm{~V}$ scan from the vertical displacement at the corresponding point of the actuated scan. Since the device was secured to the profilometer stage and not moved relative to the stylus between scans, this was accomplished easily with a spreadsheet program. A typical graph of the resulting normalized displacements is shown in Figure 5.5. 


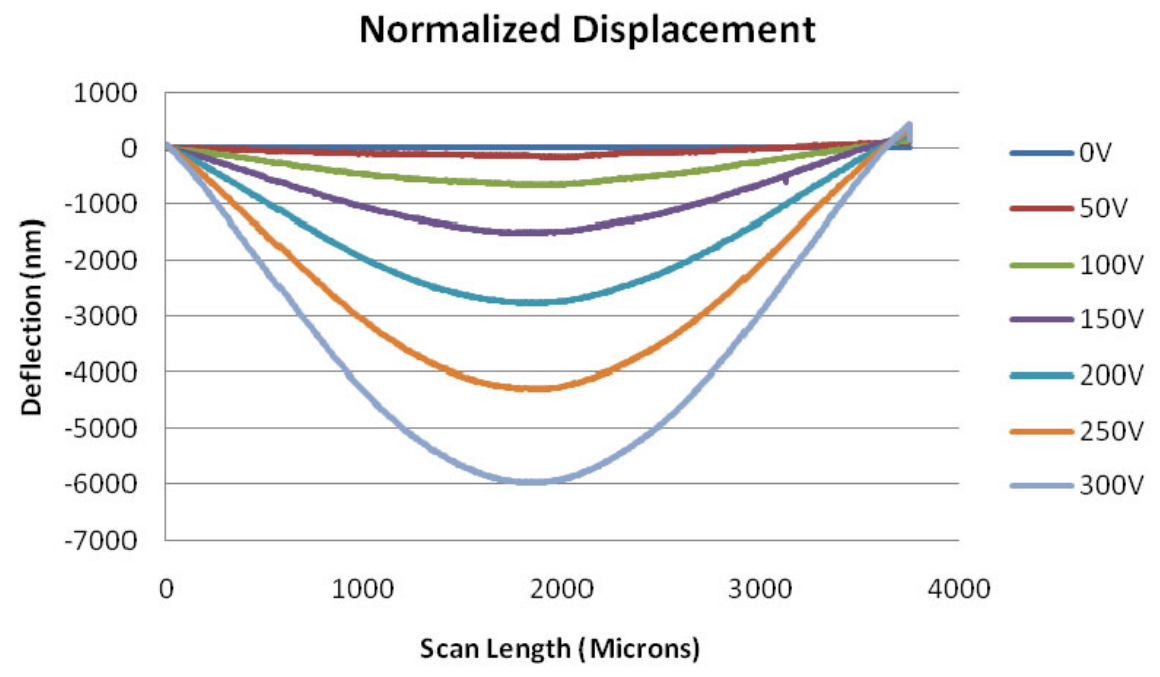

Figure 5.5: A representative normalized displacement plot.

Once this normalized deflection data was obtained, the point of maximum deflection was easy to identify and quantify. Appendix B contains the raw profilometer scans and the normalized deflection curves for each device characterized. The maximum deflection values for each scan were compiled and are presented in Appendix C. After the maximum displacement for each voltage was identified, it was plotted as a function of actuating voltage to generate voltage-displacement curves. A typical voltagedisplacement curve is shown in Figure 5.6. 


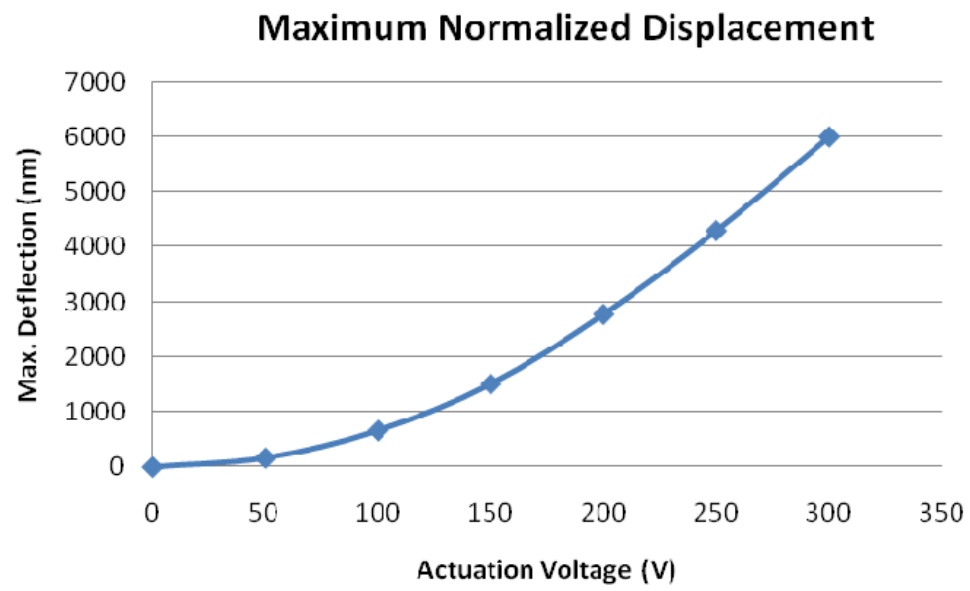

Figure 5.6: A representative plot of maximum displacement vs. actuating voltage.

These voltage-displacement curves were compiled for each test of each device and are contained in Appendix D. 


\section{SECTION 6: DISCUSSION}

\subsection{Modeling and Characterization Results}

Three separate techniques for predicting the deflection of a membrane under actuation have been discussed throughout this paper: the initial design equation, the design equation taking into consideration the non-linear electrostatic force response and software FEA. These techniques give predictions that vary widely from one another and, as will be shown, some of these predictions vary significantly from the deflections observed in actual devices.

The initial design equation [Eq. (2.9)] was developed first and is the most basic technique used in this work. It related the deflection at the center of the membrane to the flexural stiffness of the membrane and the electrostatic force causing the deflection. Its main shortcoming was that it assumed that the electrostatic force on the membrane remained constant as the membrane deformed - this is not the case, but this technique was still useful as an approximation. The nonlinear electrostatic force model [Eq. (2.16)] was an evolution of the initial design equation which considered the increase in electrostatic force as the membrane deflects. However, it assumed that the membrane remained rigid and deflected uniformly towards the counterelectrode, and, hence, the force on the membrane and the equilibrium displacement were simply functions of the electrode gap and not complicated by the geometry of the deformed membrane. This second model corrected the assumption made by the first model, but was not without an assumption of 
its own. These two shortcomings were addressed in the modeling section of this paper through the use of software FEA.

It is useful to graphically compare the predictions of all three modeling techniques with the deflection of an actual device. To accomplish this, each of the three techniques was used to predict the actuation voltage-deflection performance of a device defined by the parameters in Table 6.1. Additionally, the voltage-deflection curve of a representative device closely matching the parameters in Table 6.1 was plotted along with the predictions. These voltage-deflection curves are presented in Figure 6.1.

\begin{tabular}{|c|c|}
\hline$v$ (Poisson's ratio) & 0.28 \\
\hline$\varepsilon$ (relative dielectric permittivity) & 1 \\
\hline$\varepsilon_{0}$ (permittivity of free space) & $8.854^{*} 10^{-12} \mathrm{~F} / \mathrm{m}$ \\
\hline L (membrane side length) & $4400^{*} 10^{-6} \mathrm{~m}$ \\
\hline E of [110] Silicon (Young's modulus) & $168^{*} 10^{9} \mathrm{~N} / \mathrm{m}^{2}$ \\
\hline $\mathrm{d}$ (gap thickness) & $44^{*} 10^{-6} \mathrm{~m}$ \\
\hline $\mathrm{h}$ (membrane thickness) & $7^{*} 10^{-6} \mathrm{~m}$ \\
\hline
\end{tabular}

Table 6.1: Parameters used to model membrane deflection with hand calculations. 


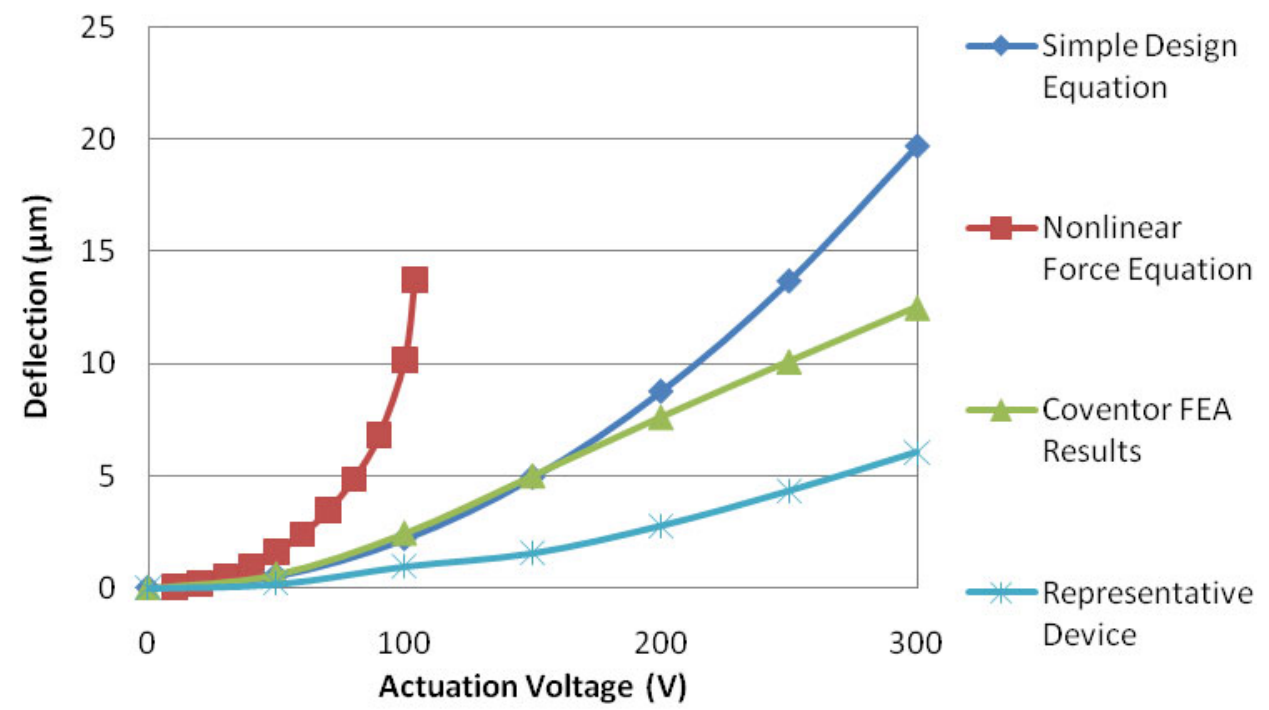

Figure 6.1: Comparison of modeling techniques to representative device data.

As Figure 6.1 shows, in their current state none of the modeling techniques accurately predicted the deflection of an actual device. This disagreement is attributed to the inaccuracies associated with precisely measuring the membrane thickness and electrode gap and the residual stresses present in the membranes. It is worth noting that with the exception of the curve for the nonlinear electrostatic force equation, each curve follows the same general quadratic relationship between deflection and actuation voltage, and these techniques differ only in the values of the $x$ and $x^{2}$ coefficients. The nonlinear electrostatic force curve behaves asymptotically, which is inconsistent with the quadratictype curves of the other techniques within the $0-300 \mathrm{~V}$ actuation voltage range. The fact that the other data display similar quadratic relationships is encouraging in spite of the disparity between the predicted and measured displacement curves. Of all the modeling techniques, the software FEA was the closest to predicting actual device performance; this is expected because of the level of sophistication of the technique. Indeed, software FEA is likely the most thorough, accurate and physically relevant 
modeling technique, especially when considering structures that experience significant deformations and the resulting non-uniform electrostatic force distribution. The accuracy of the FEA model could be improved by taking into account the residual stress gradient within the membrane when defining the properties of each individual model component.

The nonlinear electrostatic force model predicted a greater displacement for a given voltage than any other model and, in fact, predicted device pull-in at an actuation voltage of $104 \mathrm{~V}$. Obviously, this prediction was not shared by the other modeling techniques nor was pull-in observed in actual devices, even up to the maximum actuation voltage of $300 \mathrm{~V}$. This inaccuracy can be attributed to the model's treatment of the membrane as a rigid plate which displaces uniformly towards the counterelectrode; this causes the electrostatic force to increase proportionally to the inverse square of the difference between the initial gap and the displacement. In reality, the plate does not deflect uniformly and, hence, the electrostatic force is some fraction of the force predicted by the model, the magnitude of which depends on the geometry of the deformation. For these reasons, this model can be disregarded.

It is interesting to note that the simplest technique used in this work, i.e. the initial design equation, follows the same quadratic relationship as the actual device data. In fact, the initial design equation prediction differs from the device data by a simple fitting factor that changes from device to device. These fitting factors were determined by taking the average of the predicted displacement divided by the measured displacement for each actuation voltage. These fitting factors are compiled in Appendix E. Because it varies 
with device, this fitting factor is not something that could be used to improve the accuracy of the model or predict the displacement for any device other than the one for which it was calculated. However, its presence suggests that the underlying voltagedisplacement relationship within the model is correct. It is believed that these fitting factors arise from the inaccuracy of the device dimensions used in the model and the presence of residual stresses within the membrane, which could explain why the fitting factor changes from device to device. Figure 6.2 compares data from a typical device with the predictions of the simple design equation both before and after the fitting factor is introduced.

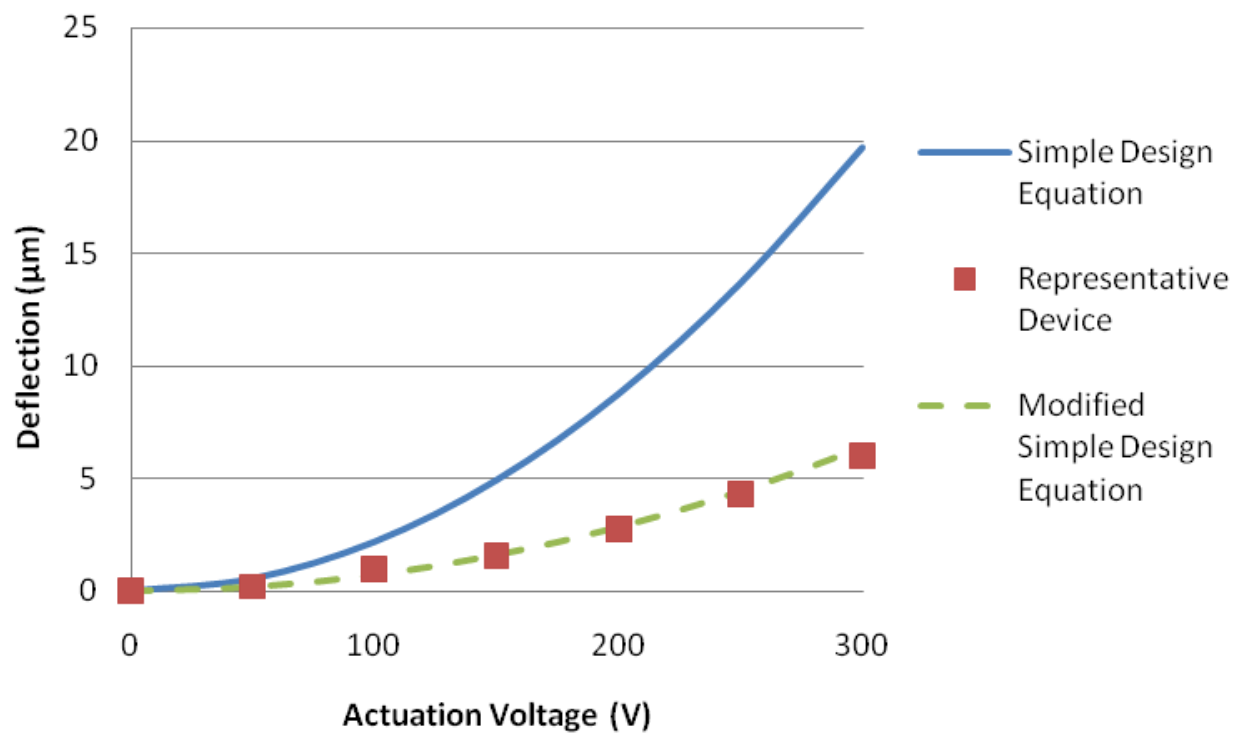

Figure 6.2: A comparison of the standard and scaled initial design equations with the measured deflection data for a representative device.

The point of introducing this fitting factor to the initial design equation was to emphasize that the measured data follows the same $y=k x^{2}$ relationship as the simple design equation, where $y$ is the deflection, $x$ is the actuation voltage, and $k$ is a factor that 
depends on geometry, material properties such as stiffness and Poisson's ratio and the dielectric medium. The accuracy of this model could be improved by obtaining more accurate measurements of the membrane and electrode gap thickness; however, the model would have to be modified to account for the presence of a residual stress. In addition, if the membrane is not parallel to the counterelectrode the electrostatic force will be stronger at one end of the membrane, and this could distort the maximum displacement measurements.

As mentioned in the previous section, one of the devices was subjected to an oscillating actuation voltage to test for device fatigue. The device deflection was characterized using DC actuation prior to testing, after $2 * 10^{6}$ cycles, and after $10^{7}$ total cycles. No significant change in deflection was observed, as shown in Figure 6.3. This is consistent with the generally accepted view that monocrystalline silicon MEMS components are not susceptible to fatigue, and is one of the advantages of MEMS components over similar macro-scale components. 


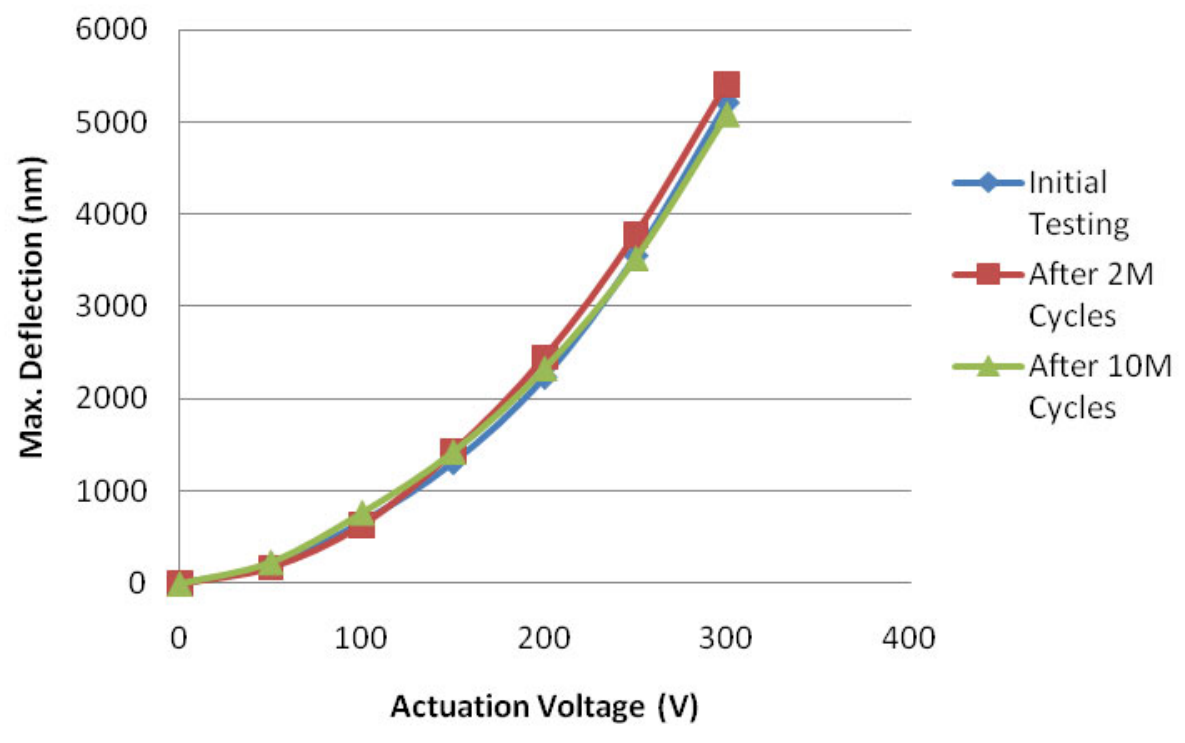

Figure 6.3: Fatigue test results.

\subsection{Fabrication Issues and Observations}

A number of observations were made during the fabrication process which could contribute to an explanation for the disparity between the expected device performance and the measured performance.

It should be noted that this work not only represents the first time that a long thermal diffusion from a spin-on dopant source was attempted in the microfabrication facility, but also the first in-house experimentation with heavily-doped boron etch-stop behavior in silicon. A literature review yielded inconsistent accounts of boron etch-stop behavior; specifically, the reported boron concentration required for etch-stop behavior ranged from $1 * 10^{19}$ atoms $/ \mathrm{cm}^{3}$ to $2 * 10^{20}$ atoms $/ \mathrm{cm}^{3}[20-21,23-26]$. Additionally, very little literature was found which discussed heavily doping silicon from spin-on boron sources; ion implantation and gas-phase doping were commonly used in the literature. The 
stability of the spin-on dopant film at such high temperatures and long diffusion times, as well as the validity of the "infinite-source" assumption, were called into question. Firsthand experience with etch-stop behavior was desired to gain a better understanding of the phenomenon and how to control it.

Wafers were heavily doped with boron using the 5-hour thermal diffusion discussed in Section 3; however, because the doping process and etch-stop behavior were not fully understood, the final membranes were measured to be thicker than the design anticipated. As Figure 6.4 shows, the boron concentration profile measured with D-SIMS does not agree with the pre-dep concentration profile for a 5 -hour thermal diffusion at $1100^{\circ} \mathrm{C}$ predicted by Fick's second law. In fact, the total dopant dose computed by integrating the area under the curve in Figure 6.4 is a factor of two greater than the dose predicted by the pre-dep model. Eq. (6.1) gives the dopant dose $Q_{0}$ as a function of the diffusion coefficient $D$, the diffusion time $t$, and the surface dopant concentration $C_{S}$.

$$
Q_{0}=\frac{2}{\sqrt{\pi}} C_{S} \sqrt{D t}
$$

This measured dose can be used to back-calculate the actual diffusion coefficient and the corresponding diffusion temperature, which was calculated to be approximately $1163^{\circ} \mathrm{C}$. The expected dopant concentration profile resulting from a 5-hour pre-dep at this temperature is also shown in Figure 6.4, and this curve is in closer agreement with the profile measured by D-SIMS. 


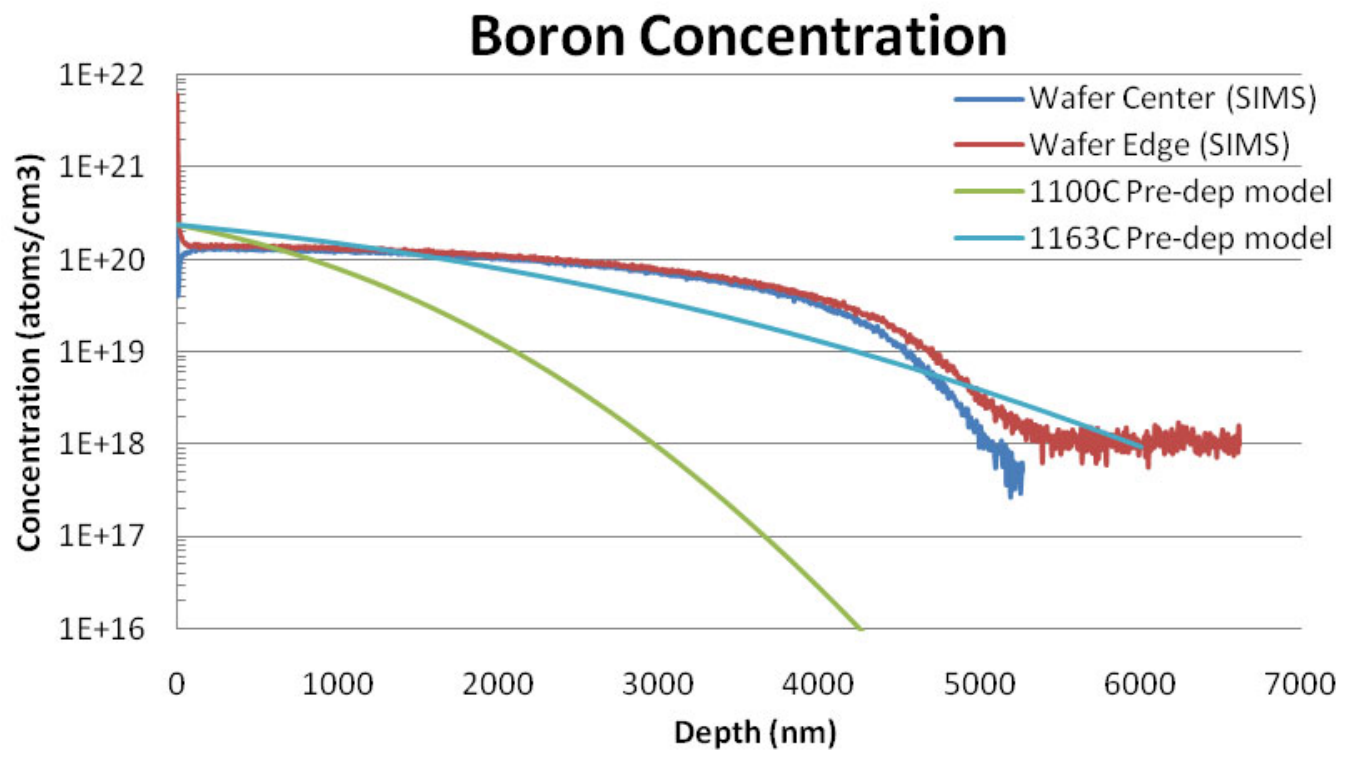

Figure 6.4: Measured and predicted boron concentrations after a 5-hr thermal diffusion.

Another complication in the fabrication process was the lack of a robust, accurate method of measuring the thickness of the membranes after fabrication. As mentioned in Section 3 , several techniques were used to measure the thickness, but none of these yielded results with high degrees of confidence. When calibrated against a step-height standard, the stylus profilometer is capable of accurately measuring etch pit depths up to several hundred microns deep. Subtracting this etch pit depth from the wafer thickness yields the membrane thickness. However, this same stylus profilometry technique cannot be used to measure the thickness of the entire wafer unless the wafer is in intimate contact with a suitable backing substrate. Any gap underneath the wafer would introduce error into the thickness measurement and subsequent calculations. C-clamp style micrometers can directly measure the thickness of the membrane, but are not accurate enough to obtain the necessary sub-micron resolution. Membranes may be separated from the surrounding chip and measured using a stylus profilometer; however, in addition to being destructive, 
this technique suffers the same drawbacks as measuring the total wafer thickness with stylus profilometry. In addition, any bowing in the membrane introduces error into the measurement. For these reasons, the thickness measurements made in Section 3 were best approximations of the membrane thickness and not exact measurements. These approximations could account for some of the difference between the modeling predictions and the measured device deflections.

In the future, optical transmission could be used to determine the thickness of silicon membranes if thickness standards are obtained. These standards could be fabricated from silicon-on-insulator wafers wherein the silicon device layer forms the membrane and has a well known thickness. These silicon-on-insulator wafers contain three separate layers. The base layer is bulk monocrystalline silicon and is referred to as the handle layer. Above the handle layer is a thin layer of insulating material, typically silicon dioxide. Above this insulating layer is the silicon device layer whose thickness can be controlled during the growth process. By etching through the so-called handle layer until the insulating layer is reached, the device layer of known thickness becomes the membrane. Optical transmission measurements could be made on a series of silicon-on-insulator wafers with device layer thicknesses ranging from, for example, $10 \mu \mathrm{m}$ to $1 \mu \mathrm{m}$. Using these known thickness standards, the optical transmission of a device membrane could be measured after etching to determine its thickness.

Finally, the bonding process where the silicon die is attached to the Pyrex wafer is still in the early phases of development, and significant variation exists in the thickness and 
uniformity of the SU-8 gap layer, as shown in Table 3.10. The reported gap height values were averages of multiple gap thickness measurements made around each silicon die. In some cases the individual measurements varied by several microns, suggesting that the silicon die and membrane were not entirely parallel to the counterelectrode. This could be due to either non-uniformities in the thickness of the SU-8 film, or an unevenly distributed load on the silicon die during the hard-bake bonding step. This electrode gap thickness variation could also cause the devices to behave differently from the modeling predictions. 


\section{SECTION 7: CONCLUSIONS AND FUTURE WORK}

This work has demonstrated that thin silicon membranes can be fabricated using the resources available within the Cal Poly, San Luis Obispo Microfabrication lab. Moreover, these structures can be actuated electrostatically and their deflection characterized and shown to be controllable and predictable. These membranes are similar to structures used extensively in the MEMS industry; the designs, processes and modeling techniques described in this work should serve as a starting point for the realization of more complex MEMS structures and devices.

Some of the processing techniques used to fabricate these devices require further development and characterization before more advanced structures can be made. The performance of these structures under actuation was simulated with a several modeling techniques. Among the techniques investigated, software-based finite element analysis was shown to be the most accurate modeling technique. The initial design equation which was derived to identify appropriate device dimensions was the most accurate handcalculation modeling technique. While there is disparity between the initial design equation predictions and the recorded data, both data sets follow the same $y=k x^{2}$ relationship between deflection and actuating voltage. The disparity between the FEA modeling predictions and the measured device performance can be attributed to measurement inaccuracies and device properties not accounted for by the model, including membrane thickness, electrode gap thickness, residual stresses and the degree of parallelism between the membrane and counterelectrode. Further development of the 
fabrication processes should bring these device properties under some degree of control, improving the agreement between the model and real devices. Once these improvements are made, the accuracy of the initial design equation and FEA modeling techniques should be reevaluated.

This project is far from completed; a significant amount of work is required before the fabrication processes are controlled. For example, the boron concentration throughout the entire thickness of the membrane could be characterized with D-SIMS. The membranes should be fabricated on low-defect-density wafers to verify the hypotheses concerning the formation of the craters. The wafers should be masked for deep etching by a material other than thermally-grown silicon dioxide to eliminate the bowing in the membranes caused by the stress of oxidation. XRD, TEM, or EBSD could be used to characterize the crystal structure of the silicon within the membrane to determine if any microstructural or crystallographic changes occurred during one of the thermal processing steps. The boron doping process and etch-stop behavior could be systematically investigated with D-SIMS to determine the relationship between the doping process and final device thickness. The etching process should be optimized to maximize etch-stop performance. A robust, accurate method of measuring membrane thickness should be developed. Finally, a device could be designed and fabricated which makes use of a membrane structure. 


\section{REFERENCES}

[1] Judy, Jack W. Microelectromechanical Systems (MEMS): Fabrication, Design and Applications. Journal of Smart Materials and Structures, Volume 10, 2001.

[2] Eddy, David S., Douglas R. Sparks. Application of MEMS Technology in Automotive Sensors and Actuators. Proceedings of the IEEE, Volume 86, Number 8, August 1998.

[3] Hsu, Tai-Ran. MEMS \& Microsystems: Design and Manufacture. New York: McGraw Hill, 2002.

[4] Maithripala, D.H.S., Jordan M. Berg, W.P. Dayawansa. Control of an Electrostatic Microelectromechanical System Using Static and Dynamic Output Feedback. Journal of Dynamic Systems, Measurement, and Control, Volume 127, September 2005.

[5] Dudley, Dana, Walter Duncan, John Slaughter. Emerging Digital Micromirror Device (DMD) Applications. Proceedings of the SPIE, Volume 4985, Number 14, 2003.

[6] Bloom, David M. Grating Light Valve: Revolutionizing Display Technology. Proceedings of the SPIE, Volume 3013, Number 165, 1997.

[7] Mehregany, Mehran, Stephen D. Senturia, Jeffrey H. Lang, Pradnya Nagarkar. Micromotor Fabrication. IEEE Transactions on Electron Devices, Volume 39, Number 9, September 1992.

[8] Onaran, A.G., M. Balantekin, W. Lee, W.L. Hughes, B.A. Buchine, R.O. Guldiken, Z. Parlak, C.F. Quate, F.L. Degertekin. A New Atomic Force Microscope Probe with Force Sensing Integrated Readout and Active Tip. Review of Scientific Instruments, Volume 77, 2006.

[9] Chavan, A.V., K.D. Wise. Batch-Processed Vacuum-Sealed Capacitive Pressure Sensors. Journal of Microelectromechanical Systems, Volume 10, Issue 4, December 2001.

[10] Mei, Tao, Wen J. Li, Yu Ge, Yong Chen, Lin Ni, Ming Ho Chan. An Integrated MEMS Three-Dimensional Tactile Sensor with Large Force Range. Sensors and Acuators A: Physical, Volume 80, 2000.

[11] Le, Hue P. Progress and Trends in Ink-Jet Printing Technology. Journal of Imaging Science and Technology, Volume 42, Number 1, February 1998.

[12] Zengerle, R., J. Ulrich, S. Kluge, M. Richter, A. Richter. A Bidirectional Silicon Micropump. Sensors and Actuators A: Physical, Volume 50, 1995. 
[13] Bao, Minhang. Analysis and Design Principles of MEMS Devices. Elsevier B.V., 2005.

[14] Liu, Chang. Foundations of MEMS. Pearson Education, Upper Saddle River, New Jersey, 2006.

[15] Tipler, Paul A., Jurgen R. Meyer-Arendt. College Physics. Worth Publishers, New York, NY, 1987.

[16] Paschen, Friedrich. Ueber die zum Funkenübergang in Luft, Wasserstoff und Kohlensäure bei verschiedenen Drucken erforderliche Potentialdifferenz. Annalen der Physik, Volume 273, Issue 5, 1889.

[17] Yi, T., L. Li, C.J. Kim. Microscale Material Testing of Single Crystalline Silicon: Process Effects on Surface Morphology and Tensile Strength. Sensors and Actuators A: Physical, Volume 83, Numbers 1-3, 2000.

[18] Wortman, J.J., R.A. Evans. Young's Modulus, Shear Modulus, and Poisson's Ratio in Silicon and Germanium. Journal of Applied Physics, Volume 36, Number 1, 1965.

[19] Brantley, W.A. Calculated Elastic Constants for Stress Problems Associated with Semiconductor Devices. Journal of Applied Physics, Volume 44, 1973.

[20] Iliescu, Ciprian, Mihaela Carp, Jianmin Miao, Francis E.H. Tay, Daniel P. Poenar. Analysis of Highly Doping With Boron From Spin-On Diffusing Source. Surface \& Coatings Technology, Volume 192, 2008.

[21] Manea, Elena, Raluca Muller, Alina Popescu. Some Particular Aspects of the Thin Membrane by Boron Diffusion Process. Sensors and Actuators A: Physical, Volume 74, 1999.

[22] Wolf, Stanley. Microchip Manufacturing. Lattice Press, Sunset Beach, CA, 2004.

[23] Palik, E.D., J.W. Faust Jr., H.F. Gray, R.F. Greene. Study of the Etch-Stop Mechanism in Silicon. Journal of the Electrochemical Society: Solid-State Science and Technology, Volume 129, Number 9, 1982. Pp. 2051-2059.

[24] Seidel, H., L. Csepregi, A. Heuberger, H. Baumgärtel. Anisotropic Etching of Crystalline Silicon in Alkaline Solutions II: Influence of Dopants. Journal of the Electrochemical Society, Volume 137, Number 11, November 1990. Pp. 36263632 .

[25] Kovacs, Gregory T.A., Nadim I. Maluf, Kurt E. Petersen. Bulk Micromachining of Silicon. Proceedings of the IEEE, Volume 86, Number 8, August 1998. 
[26] Steinsland, Elin, Martin Nese, Anders Hanneborg, Ralph W. Bernstein, Halle Sandmo, Gjermund Kittilsland. Boron Etch-Stop in TMAH Solutions. Sensors and Actuators A: Physical, Volume 54, 1996. Pp. 728-732.

[27] Seidel, H., L. Csepregi, A. Heuberger, H. Baumgärtel. Anisotropic Etching of Crystalline Silicon in Alkaline Solutions I: Orientation Dependence and Behavior of Passivation Layers. Journal of the Electrochemical Society, Volume 137, Number 11, November 1990. Pp. 3612-3625.

[28] H. Seidel. The Mechanism of Anisotropic, Electrochemical Silicon Etching in Alkaline Solutions. $4^{\text {th }}$ Technical Digest, IEEE. Solid-State Sensor and Actuator Workshop, 1990.

[29] Shikida, Mitsuhiro, Kazuo Sato, Kenji Tokoro, Daisuke Uchikawa. Differences in Anisotropic Etching Properties of KOH and TMAH Solutions. Sensors and Actuators, Volume 80, 2000. Pp. 179-188.

[30] Zubel, Irena, Irena Barycka, Kamilla Kotowska, Malgorzata Kramkowska. Silicon Anisotropic Etching in Alkaline Solutions IV: The Effect of Organic and Inorganic Agents on Silicon Anisotropic Etching Process. Sensors and Actuators A: Physical, Volume 87, 2001. Pp. 163-171.

[31] Tabata, O., Ryouji Asahi, Hirofumi Funabashi, Keiichi Shimaoka, Susumu Sugiyama. Anisotropic Etching of Silicon in TMAH Solutions. Sensors and Actuators A: Physical, Volume 34, Issue 1, 1992.

[32] Li, Sheng, Carl B. Freidhoff, Robert M. Young, Reza Ghodssi. Fabrication of Micronozzles Using Low-Temperature Wafer-Level Bonding with SU-8. Journal of Micromechanics and Microengineering, Volume 13, 2003.

[33] See http://www.siliconquest.com/.

[34] See http://www.transene.com/.

[35] Deal., B.E., A.S. Grove. General Relationship for the Thermal Oxidation of Silicon. Journal of Applied Physics, Volume 36, 1965.

[36] Chen, Ping-Hei, Hsin-Yah Peng, Chia-Ming Hsieh, Minking K. Chyu. The Characteristic Behavior of TMAH Water Solution for Anisotropic Etching on Both Silicon Substrate and $\mathrm{SiO}_{2}$ Layer. Sensors and Actuators A: Physical, Volume 93, 2001.

[37] Merlos, A., M. Acero, M.H. Bao, J. Bausells, J. Esteve. TMAH/IPA Anisotropic Etching Characteristics. Sensors and Actuators A: Physical, Volumes 37-38, 1993. 
[38] Sundaram, Kalpathy B., Arun Vijayakumar, Ganesh Subramanian. Smooth Etching of Silicon Using TMAH and Isopropyl Alcohol for MEMS Applications. Microelectronic Engineering, Volume 77, 2005.

[39] Cheng, Di, Miguel A. Gosalvez, Tatsuya Hori, Kazuo Sato, Mitsuhiro Shikida. Improvements in Smoothness of Anisotropically Etched Silicon Surfaces: Effects of Surfactant and TMAH Concentrations. Sensors and Actuators A, Volume 125, 2006. Pp. 415-421.

[40] Schnakenberg, U., W. Benecke, P. Lange. TMAHW Etchants for Silicon Micromachining. Technical Digest of Transducers '91, San Francisco, USA, June 1991. Pp. 815-818.

[41] Hertzberg, Richard W. Deformation and Fracture Mechanics of Engineering Materials, $4^{\text {th }}$ Edition. New Jersey: John Wiley \& Sons, 1996. Pg. 70.

[42] Maurits, Jan E. A. SOS Wafers - Some Comparisons to Silicon Wafers. IEEE Transactions on Electron Devices, Volume ED-25, Number 8, August 1978. Pp. 859-863.

[43] Frank, F.C., W.T. Read. Multiplication Processes for Slow Moving Dislocations. Physical Review, Volume 79, 1950. Pp. 722-723.

[44] Dash, W.C. Copper Precipitation on Dislocations in Silicon. Journal of Applied Physics, Volume 27, Number 10, 1956. Pp. 1193-1195.

[45] Secco d'Aragona, F. Dislocation Etch for (100) Planes in Silicon. Journal of the Electrochemical Society, Volume 119, Number 7, 1972. Pp. 948-951.

[46] Schimmel, D.G. Defect Etch for $<100>$ Silicon Evaluation. Journal of the Electrochemical Society, Volume 126, Number 3, 1979. Pp. 479-483.

[47] Jenkins, M.W. A New Preferential Etch for Defects in Silicon Crystals. Journal of the Electrochemical Society, Volume 131, Number 5, 1984. Pp. 1140-1145.

[48] Yang, Fuqian, Peixing Fei. Indentation Behavior of Silicon Wafer Etched by KOH. Electrochemical and Solid-State Letters, Volume 8, Number 2, 2005. Pp. G51G53.

[49] Van Veenendaal, E., K. Sato, M. Shikida, J. van Suchtelen. Micromorphology of Single Crystalline Silicon Surfaces During Anisotropic Wet Chemical Etching in KOH and TMAH. Sensors and Actuators A: Physical, Volume 93, 2001. Pp. 219-231. 
[50] Van Veenendaal, E., K. Sato, M. Shikida, A.J. Nijdam, J. van Suchtelen. Micromorphology of Single Crystalline Silicon Surfaces During Anisotropic Wet Chemical Etching in KOH: Velocity Source Forests. Sensors and Actuators A: Physical, Volume 93, 2001. Pp. 232-242.

[51] Hurle, D.T.J., P. Rudolph. A Brief History of Defect Formation, Segregation, Faceting, and Twinning in Melt-Grown Semiconductors. Journal of Crystal Growth, Volume 264, 2004. Pp. 550-564.

[52] Shikida, Mitsuhiro, Koji Kawasaki, Kazuo Sato, Yasuo Ishihara, Hiroshi Tanaka, Akihito Matsumoro. Nano-Mechanical Method for Seeding Circular-Shaped Etch Pits on (100) Silicon Surface. Sensors and Materials, Volume 15, Number 1, 2003. Pp. 21-35.

[53] Maseeh, Fairborz, Stephen D. Senturia. Plastic Deformation of Highly Doped Silicon. Sensors and Actuators, A21-A23, 1990. Pp. 861-865.

[54] Ning, X.J. Distribution of Residual Stresses in Boron Doped $\mathrm{p}+$ Silicon Films. Journal of the Electrochemical Society, Volume 143, Number 10, 1996. Pp. 3389-3393.

[55] Sokilov, V.I., N.A. Fedorovich. Mechanical Stresses on the Si-SiO2 Interface. Physica Status Solidi (a), Volume 99, 1987. Pp. 151-158. [61] Microchem Processing Guidelines for SU-8 2025-2075. Available at http://www.microchem.com. Accessed November 2008.

[56] Jaccodine, R.J., W.A. Schlegel. Measurement of Strains at Si-SiO2 Interface. Journal of Applied Physics, Volume 37, Number 6, 1966. Pp. 2429-2434.

[57] Williams, Kirt R., Kishan Gupta, Matthew Masilik. Etch Rates for Micromachining Processing_Part II. Journal of Microelectromechanical Systems, Volume 12, Number 6, 2003. Pp. 761-778.

[58] Palik, E.D., V.M. Bermudez, O.J. Glembocki. Ellipsometric Study of the Etch-Stop Mechanism in Heavily Doped Silicon. Journal of the Electrochemical Society: Solid-State Science and Technology, Volume 132, Number 1, January 1985. Pp. 135-141.

[59] Microchem Processing Guidelines for SU-8 2025-2075. Available at http://www.microchem.com. Accessed November 2008.

[60] See http://www.coventor.com. 


\section{APPENDIX A: Lithography Masks}

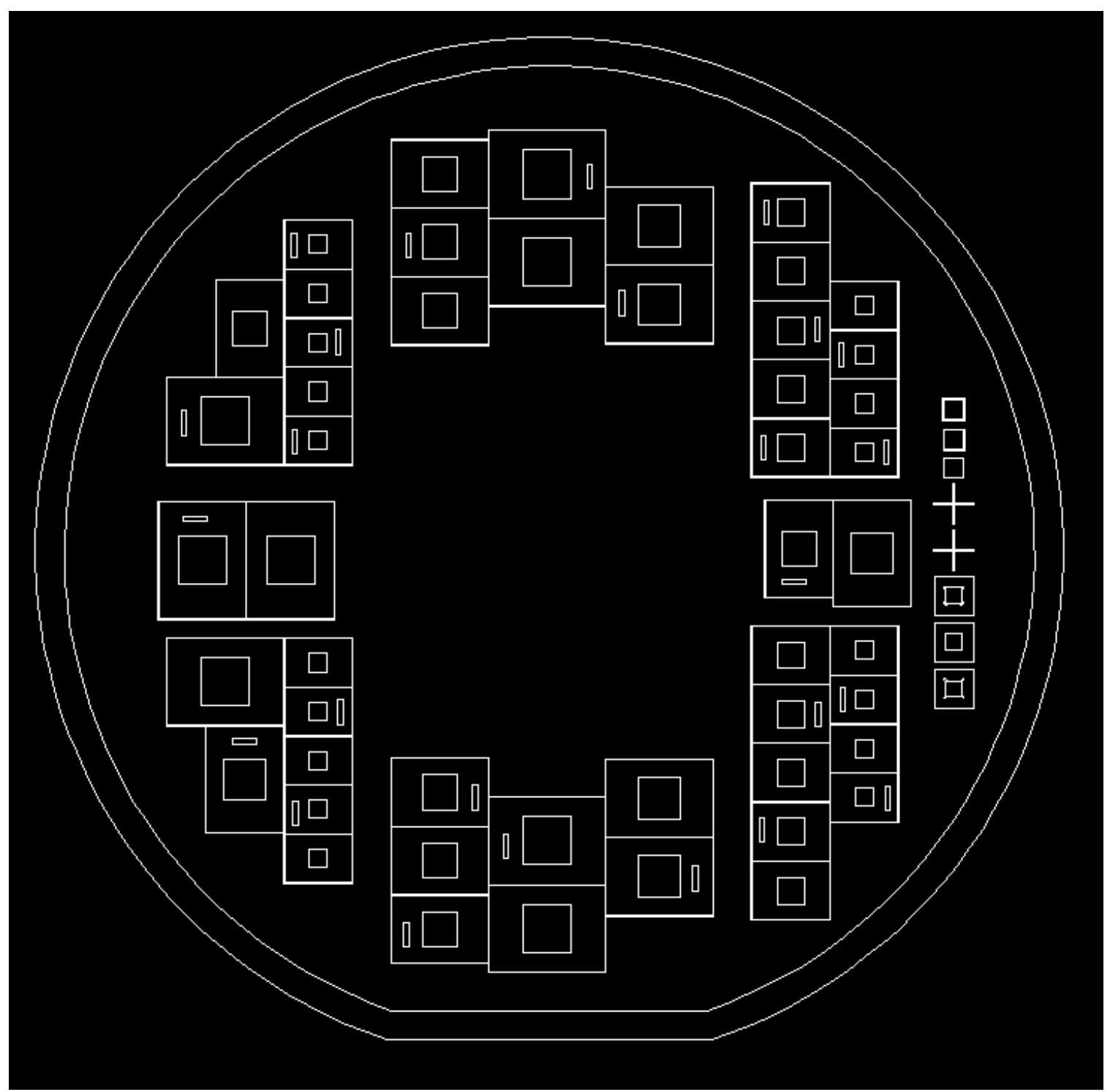

The lithography mask used to pattern the thermal oxide with etch windows for anisotropic etching. This mask contained features for five different membrane sizes: $1.3 \mathrm{~mm}, 2.2 \mathrm{~mm}, 3.0 \mathrm{~mm}, 3.7 \mathrm{~mm}$, and $4.4 \mathrm{~mm}$. $50 \mu \mathrm{m}$ trenches separate the individual dies from each other. 


\section{APPENDIX A Cont'd}

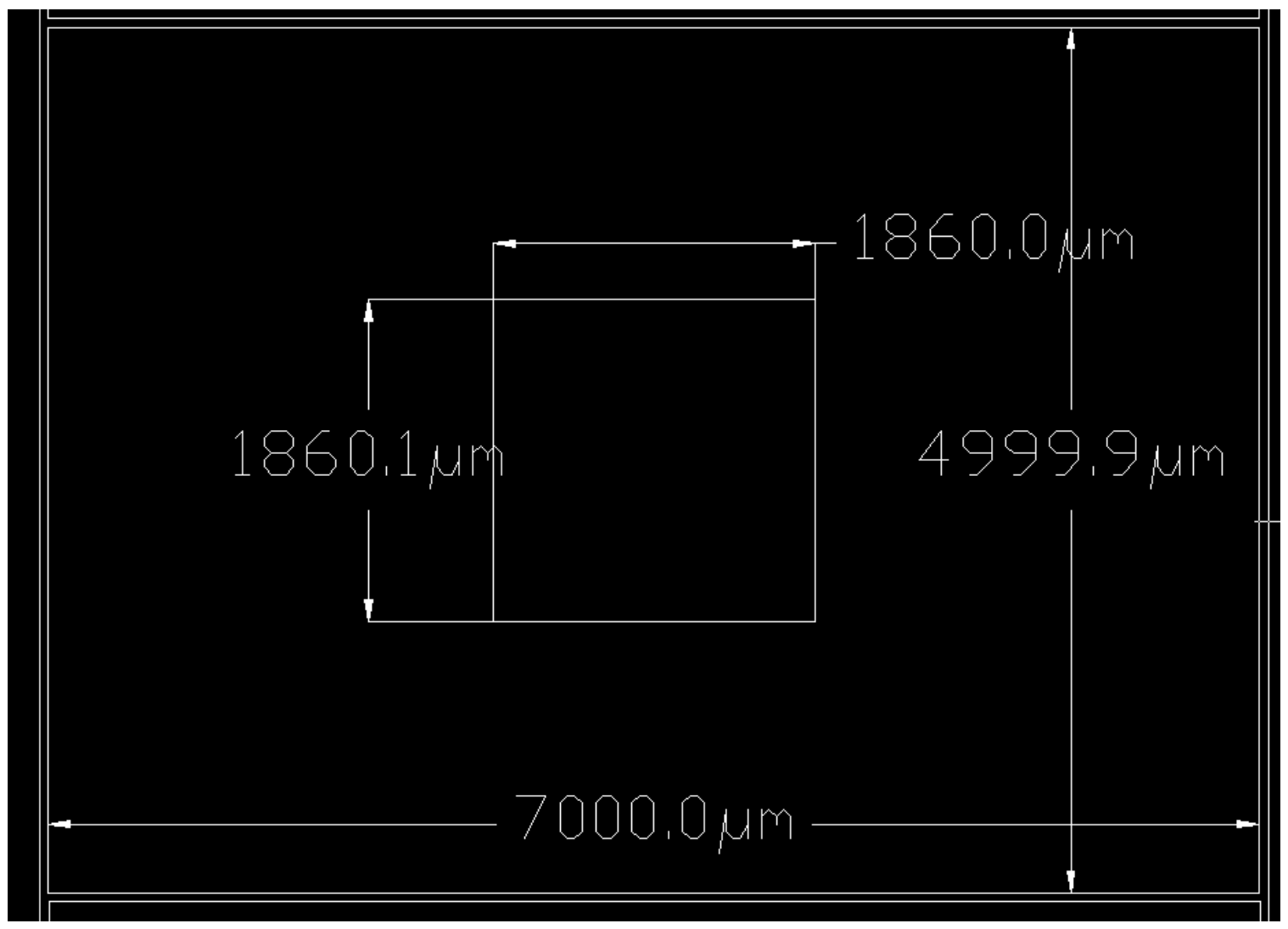

A close-up of the pattern for a $1.3 \mathrm{~mm}$ membrane. Note the $50 \mu \mathrm{m}$ trench surrounding the die. 


\section{APPENDIX A Cont'd}

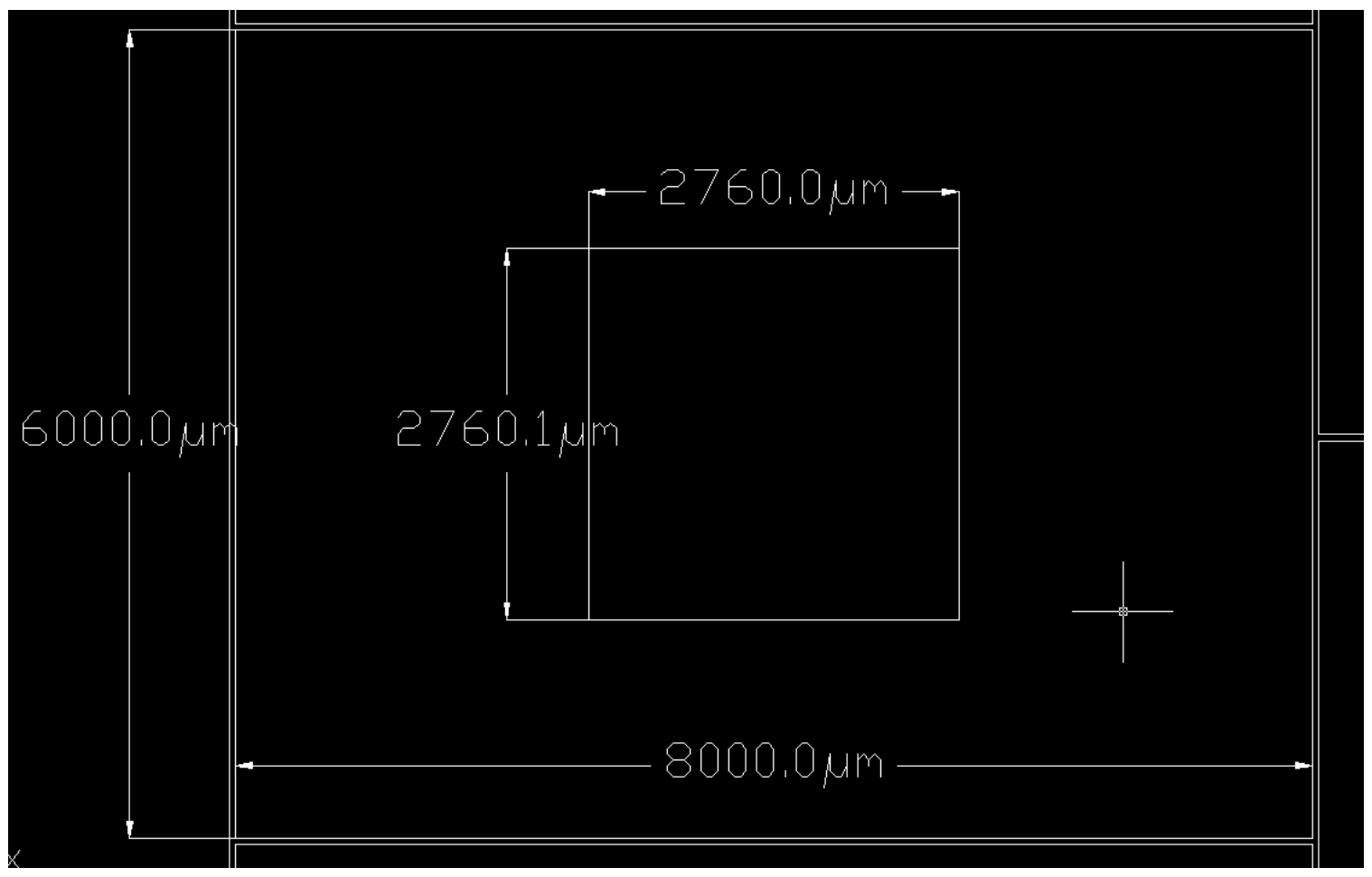

A close-up of the mask feature for a $2.2 \mathrm{~mm}$ membrane. 
APPENDIX A Cont'd

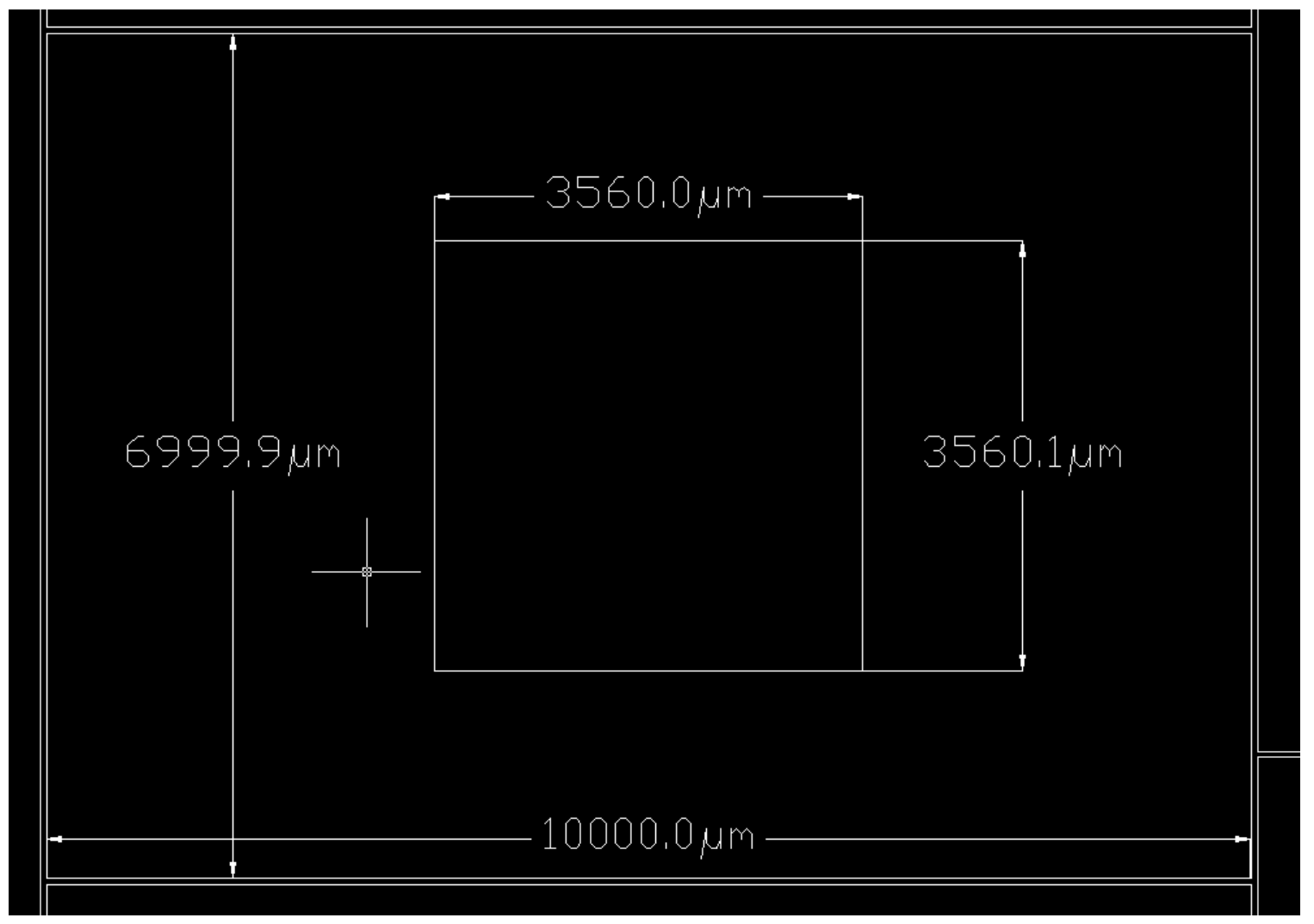

The mask feature for creating a 3.0mm membrane. 


\section{APPENDIX A Cont'd}

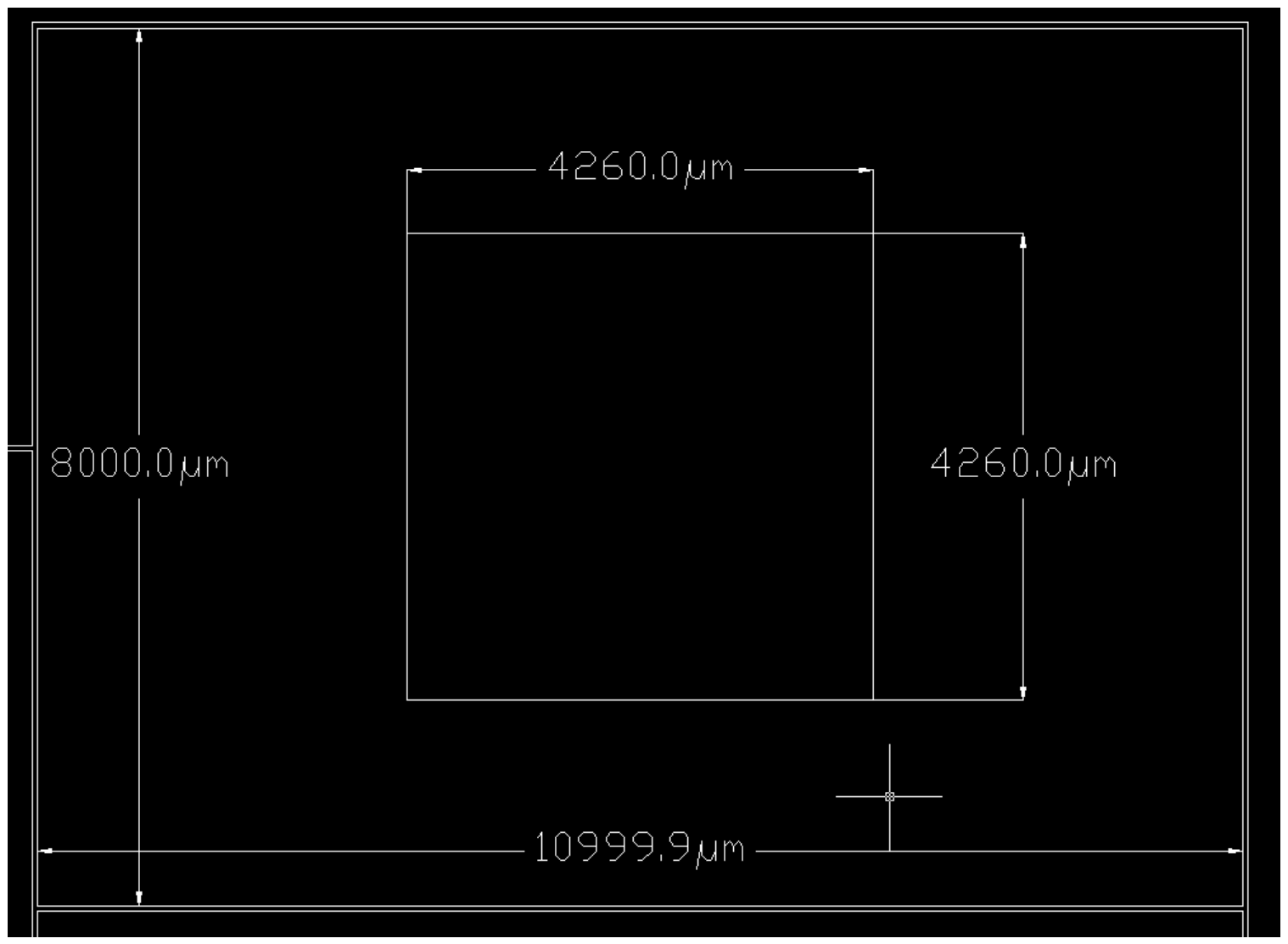

The mask feature resulting in $3.7 \mathrm{~mm}$ membranes. 


\section{APPENDIX A Cont'd}

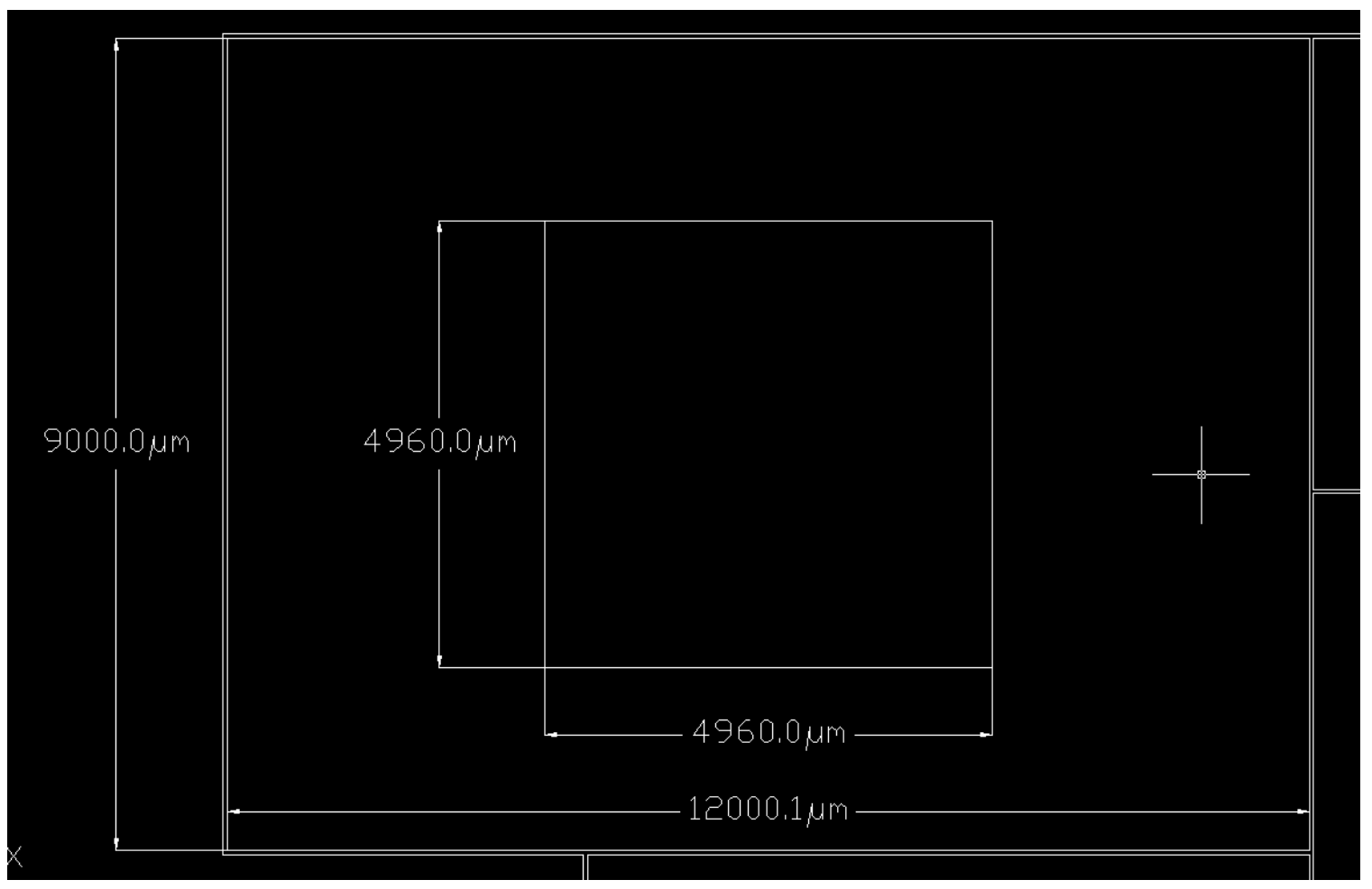

The mask feature for patterning a $4.4 \mathrm{~mm}$ membrane. 


\section{APPENDIX A Cont'd}

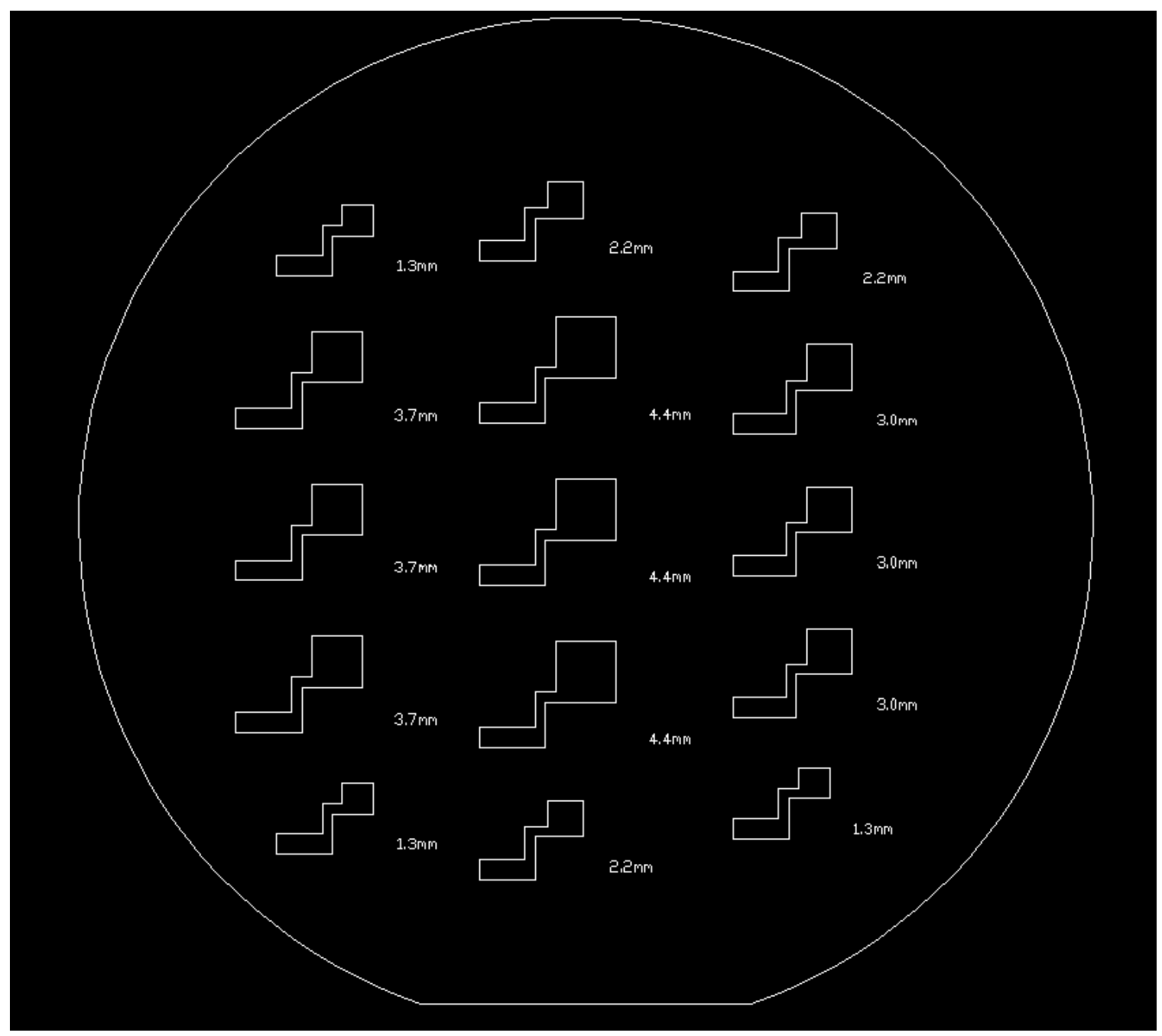

The lithography mask for patterning the aluminum counterelectrodes. 


\section{APPENDIX A Cont'd}

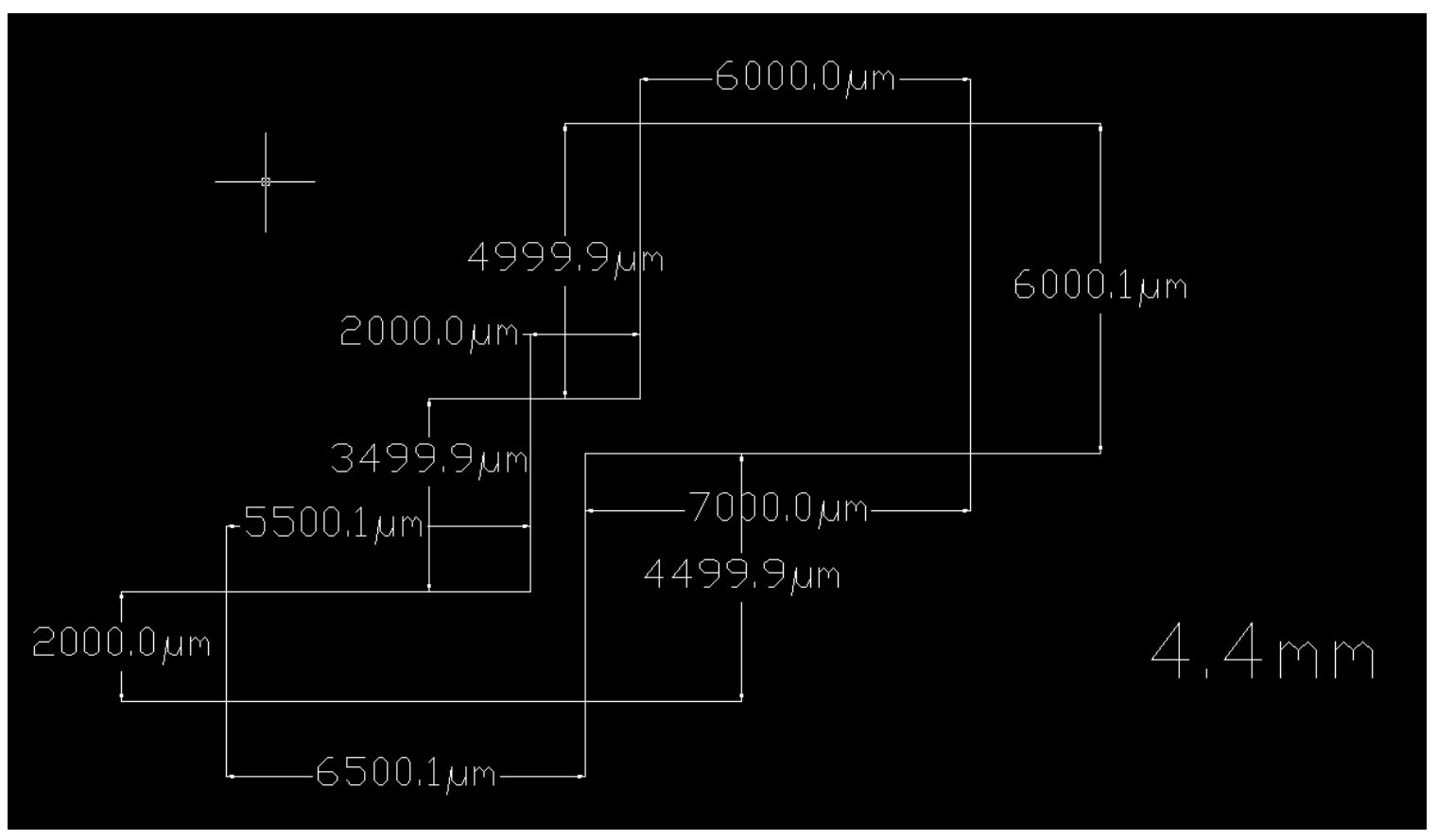

An enlarged view of a pattern for a counterelectrode corresponding to a $4.4 \mathrm{~mm}$ membrane. Dimensions are in microns and are marked on the image. 


\section{APPENDIX A Cont'd}

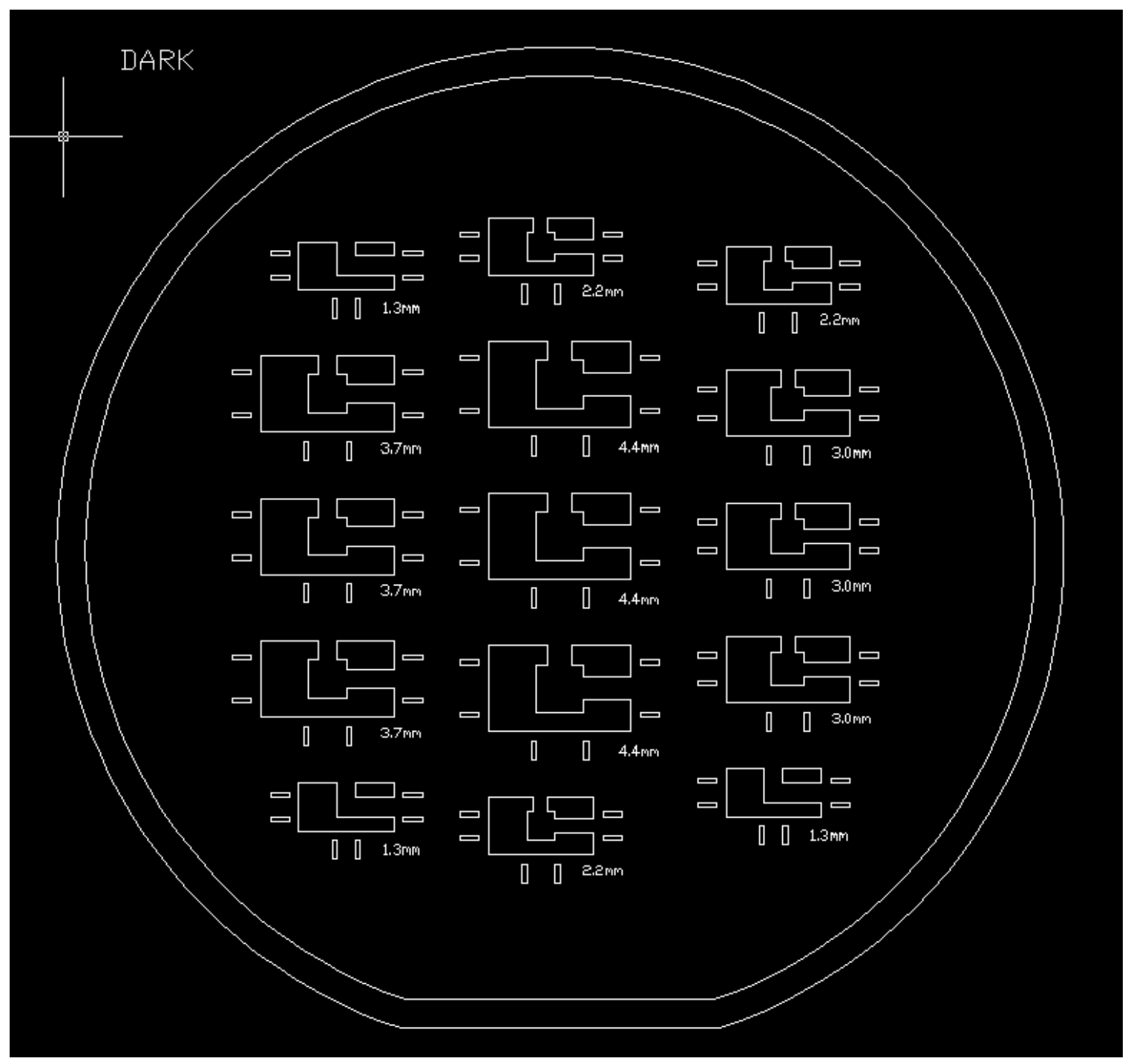

The lithography mask used to pattern the SU-8 film to create the electrode gap. 


\section{APPENDIX A Cont'd}

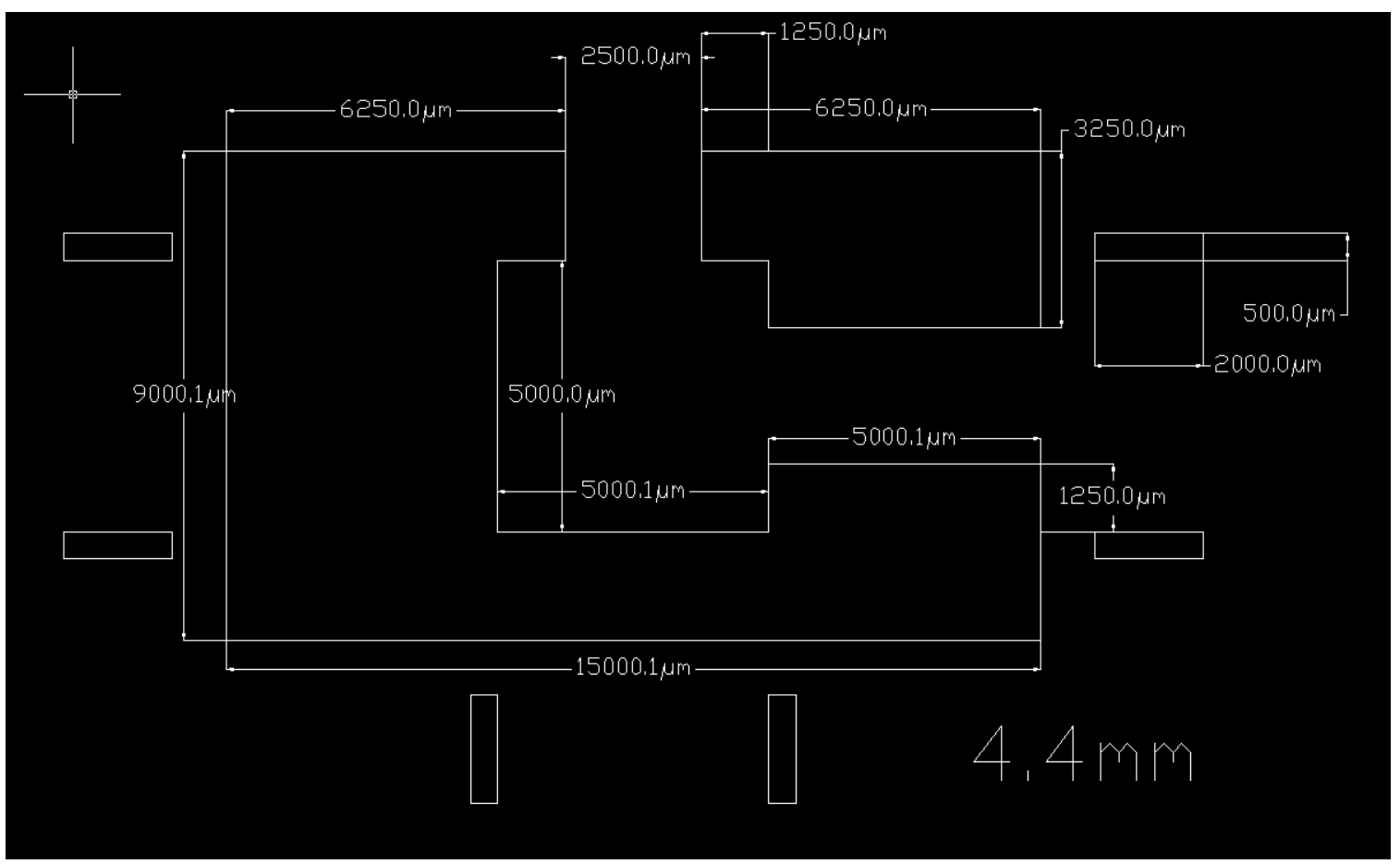

A close-up of a gap feature from the SU-8 mask. This feature is paired to a $4.4 \mathrm{~mm}$ membrane; dimensions in microns are included in the image. 


\section{APPENDIX B: Raw and Normalized Deflection}

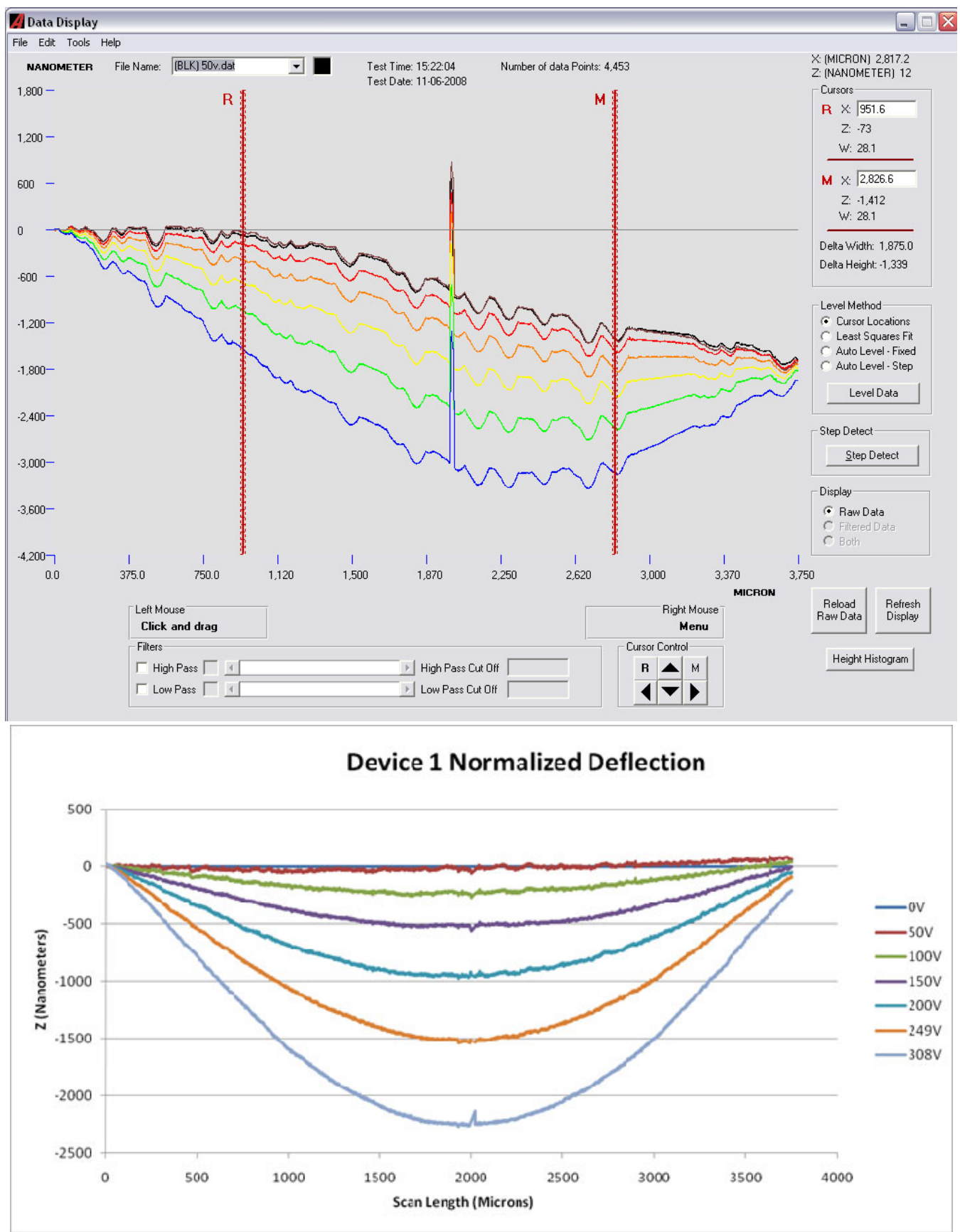

Raw and Normalized Deflection Data for Device 1, Test 1 


\section{APPENDIX B}

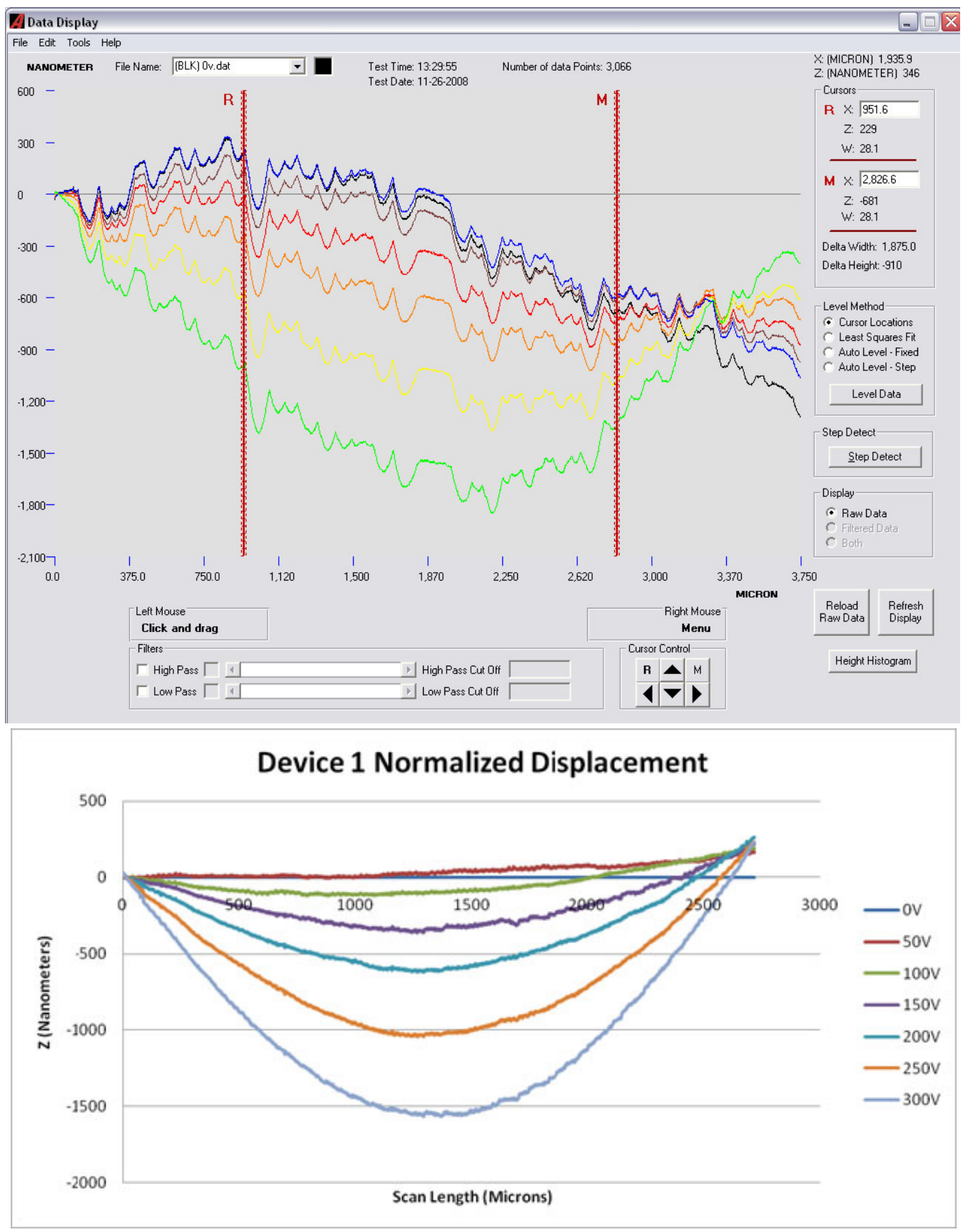

Raw and Normalized Deflection Data for Device 1, Test 2 


\section{APPENDIX B}

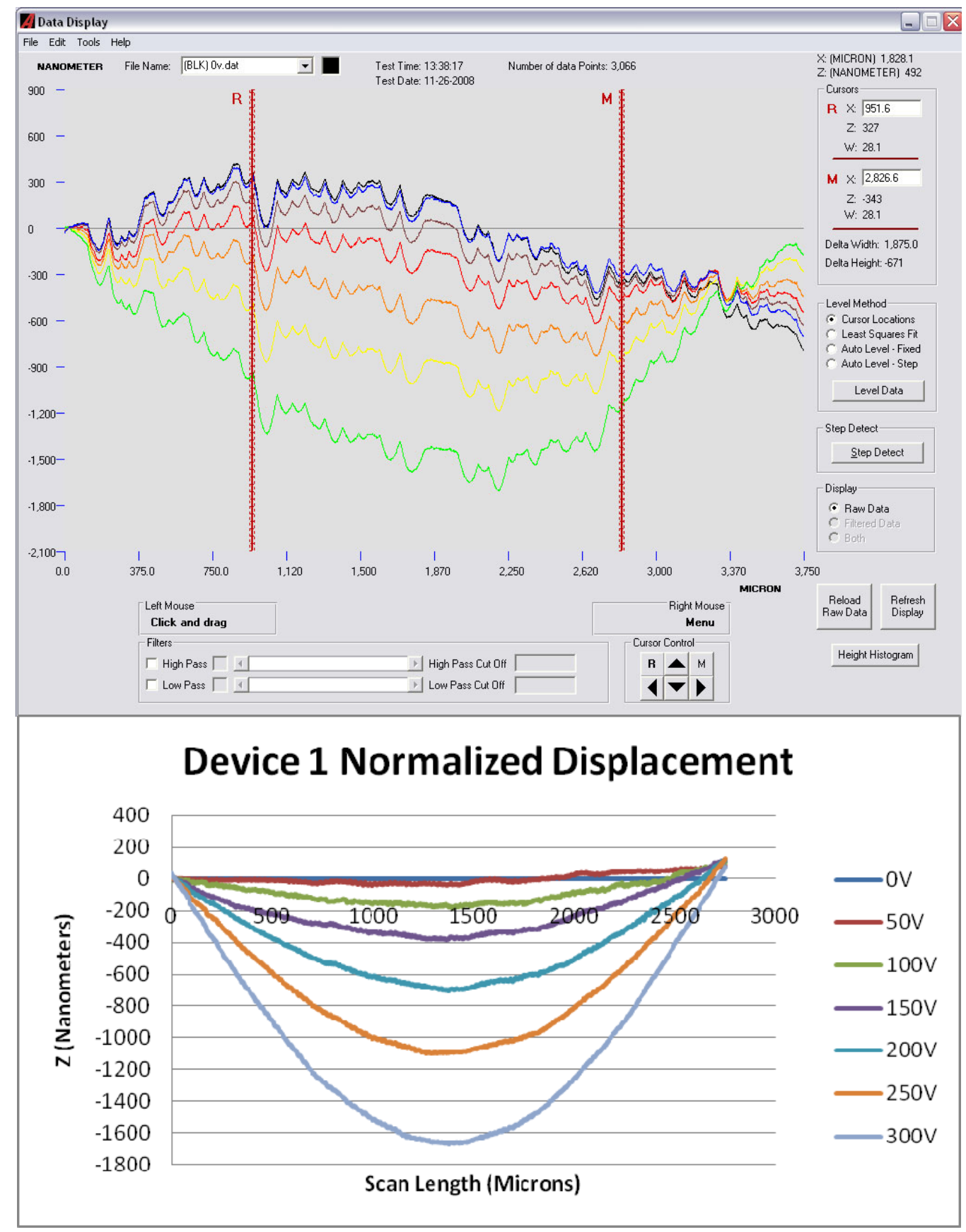

Raw and Normalized Deflection Data for Device 1, Test 3 


\section{APPENDIX B Cont'd}

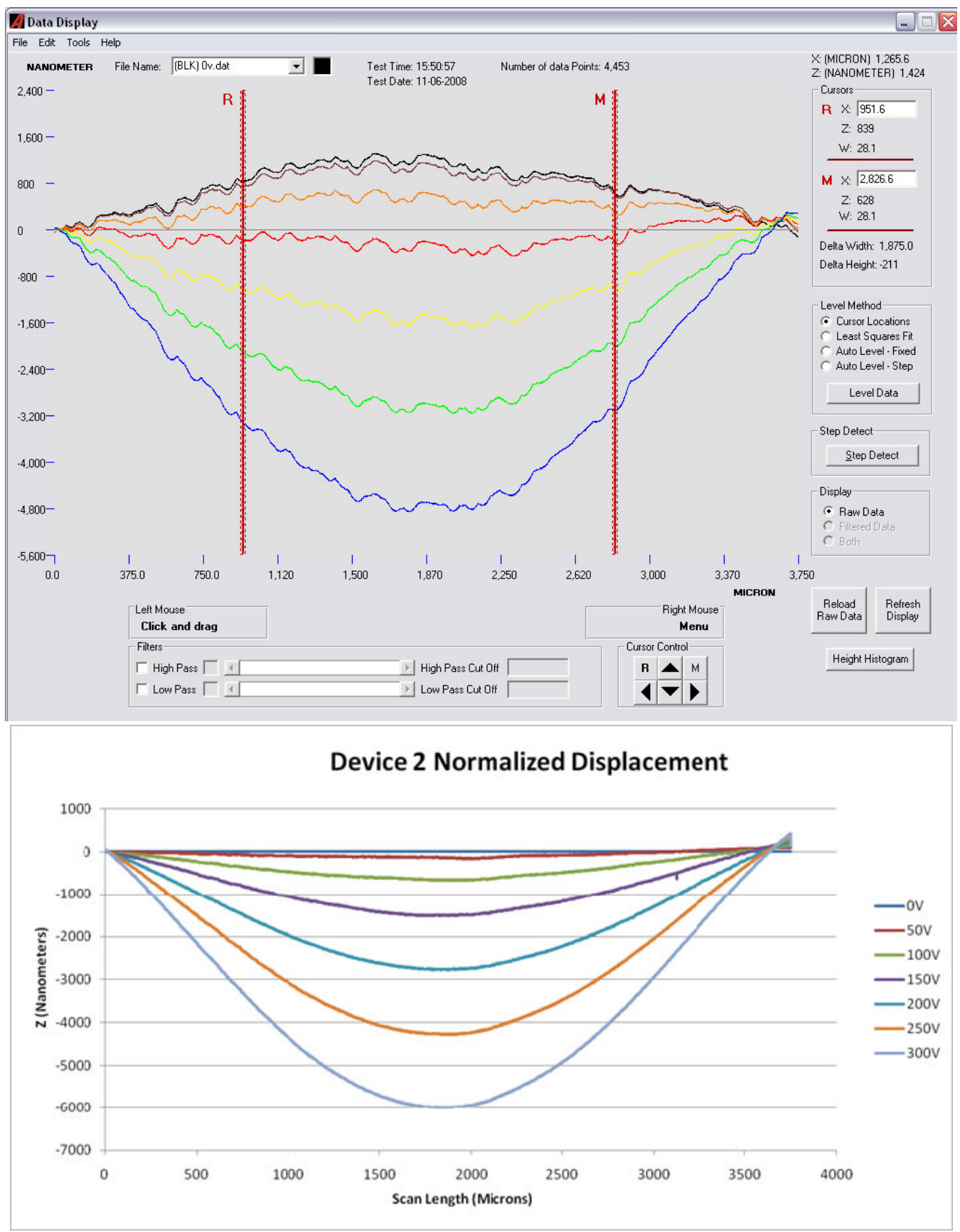

Raw and Normalized Deflection Data for Device 2, Test 1 


\section{APPENDIX B Cont'd}

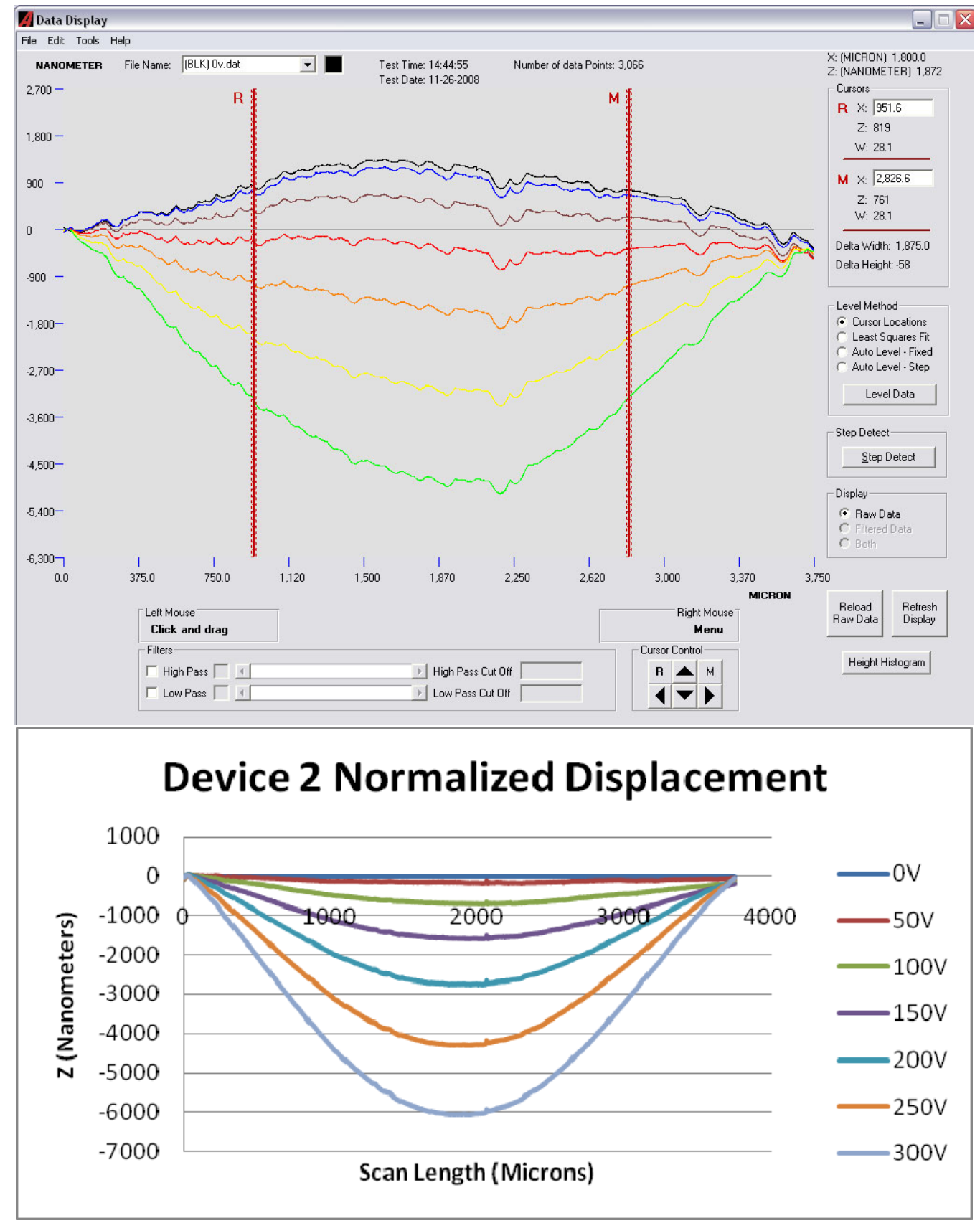

Raw and Normalized Deflection Data for Device 2, Test 2 


\section{APPENDIX B Cont'd}

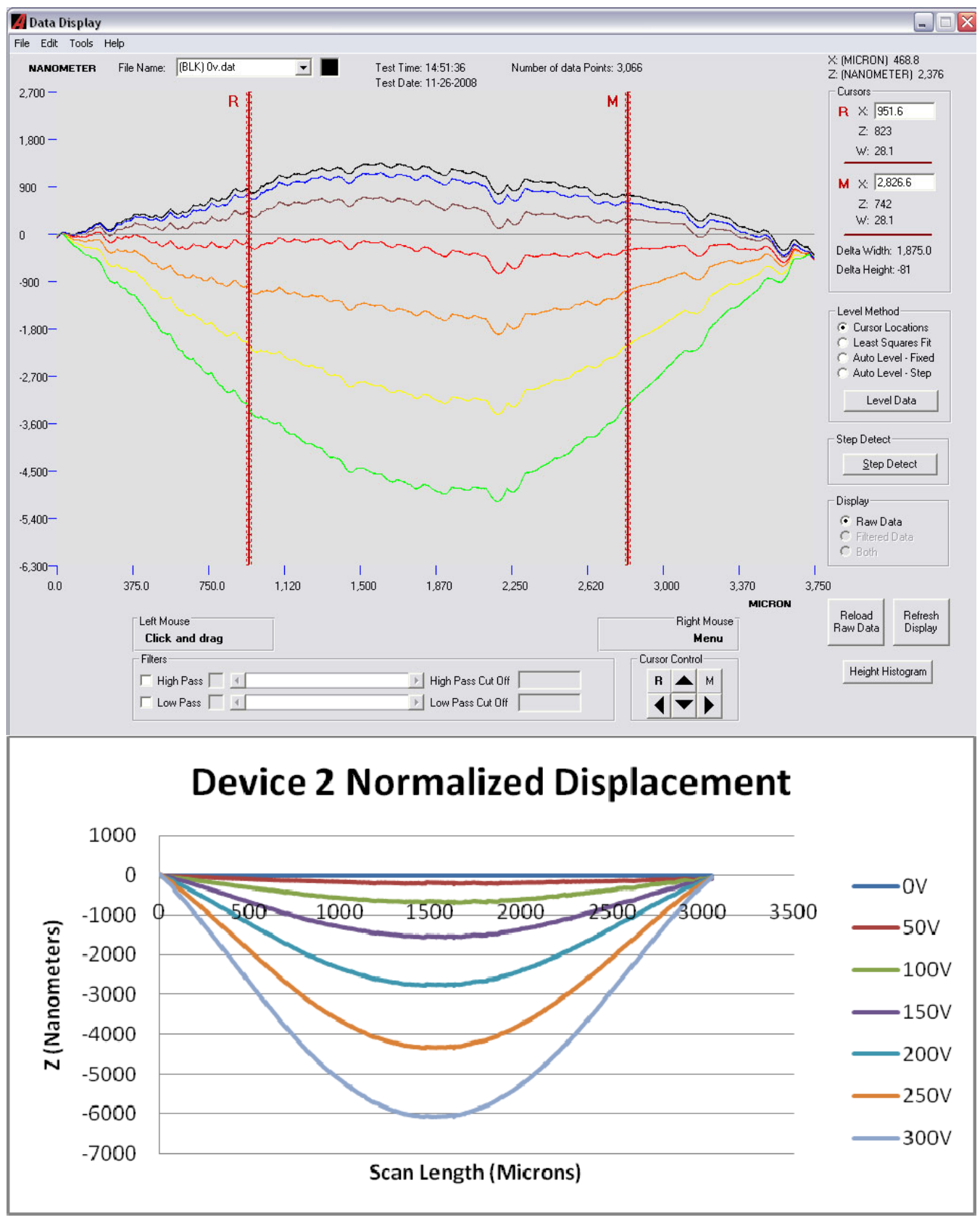

Raw and Normalized Deflection Data for Device 2, Test 3 


\section{APPENDIX B Cont'd}

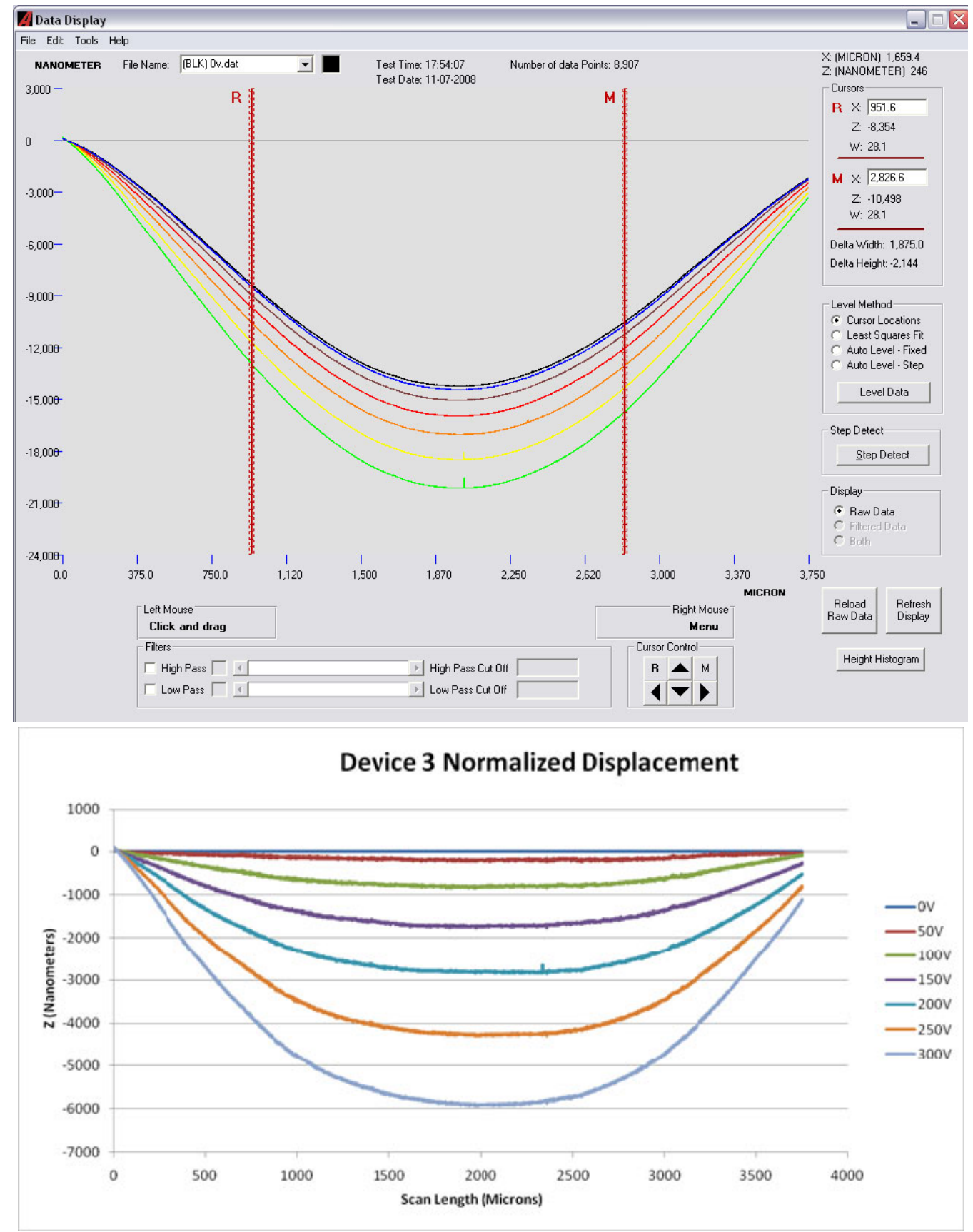

Raw and Normalized Deflection Data for Device 3, Test 1 


\section{APPENDIX B Cont'd}

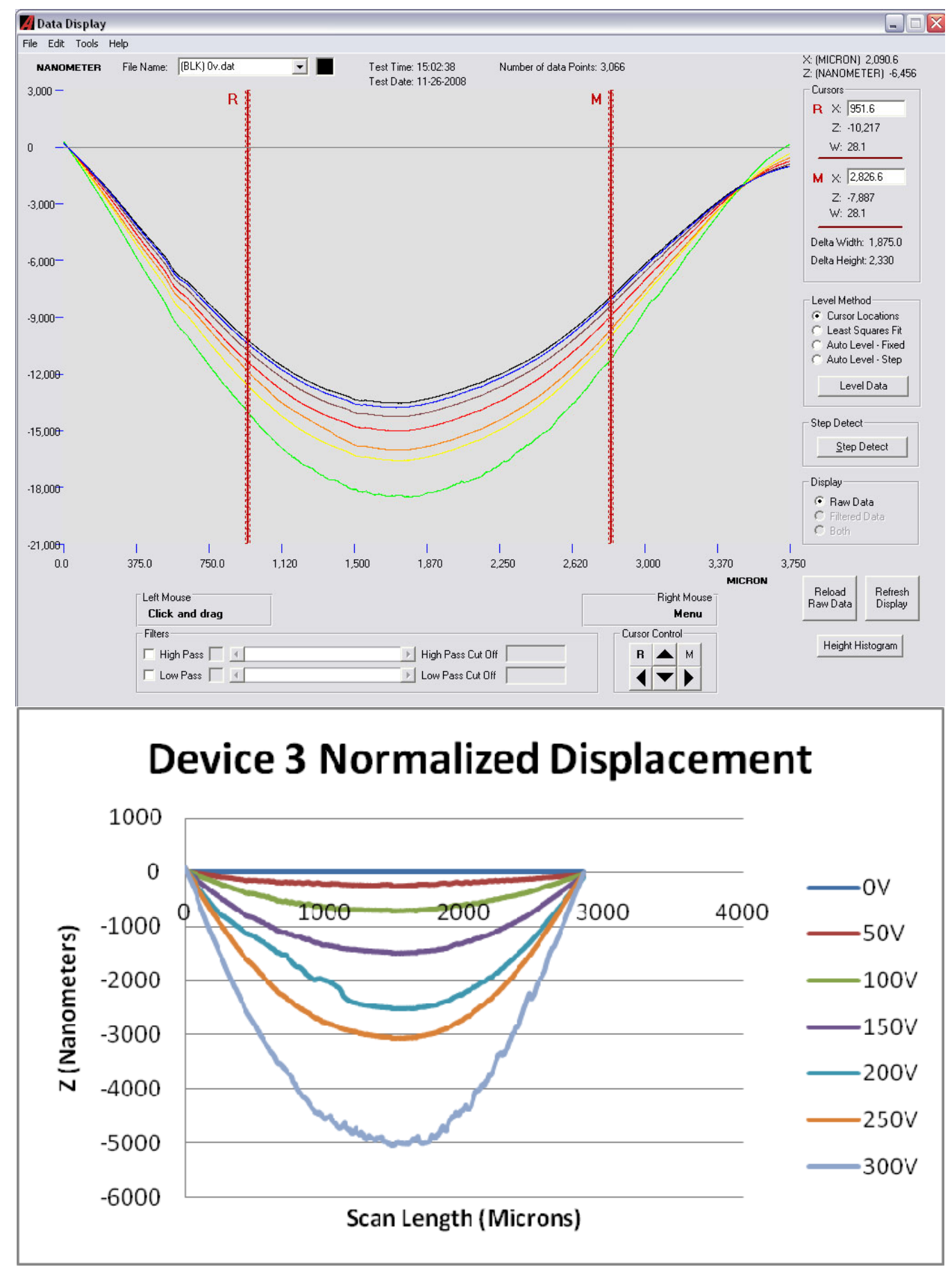

Raw and Normalized Deflection Data for Device 3, Test 2 


\section{APPENDIX B Cont'd}

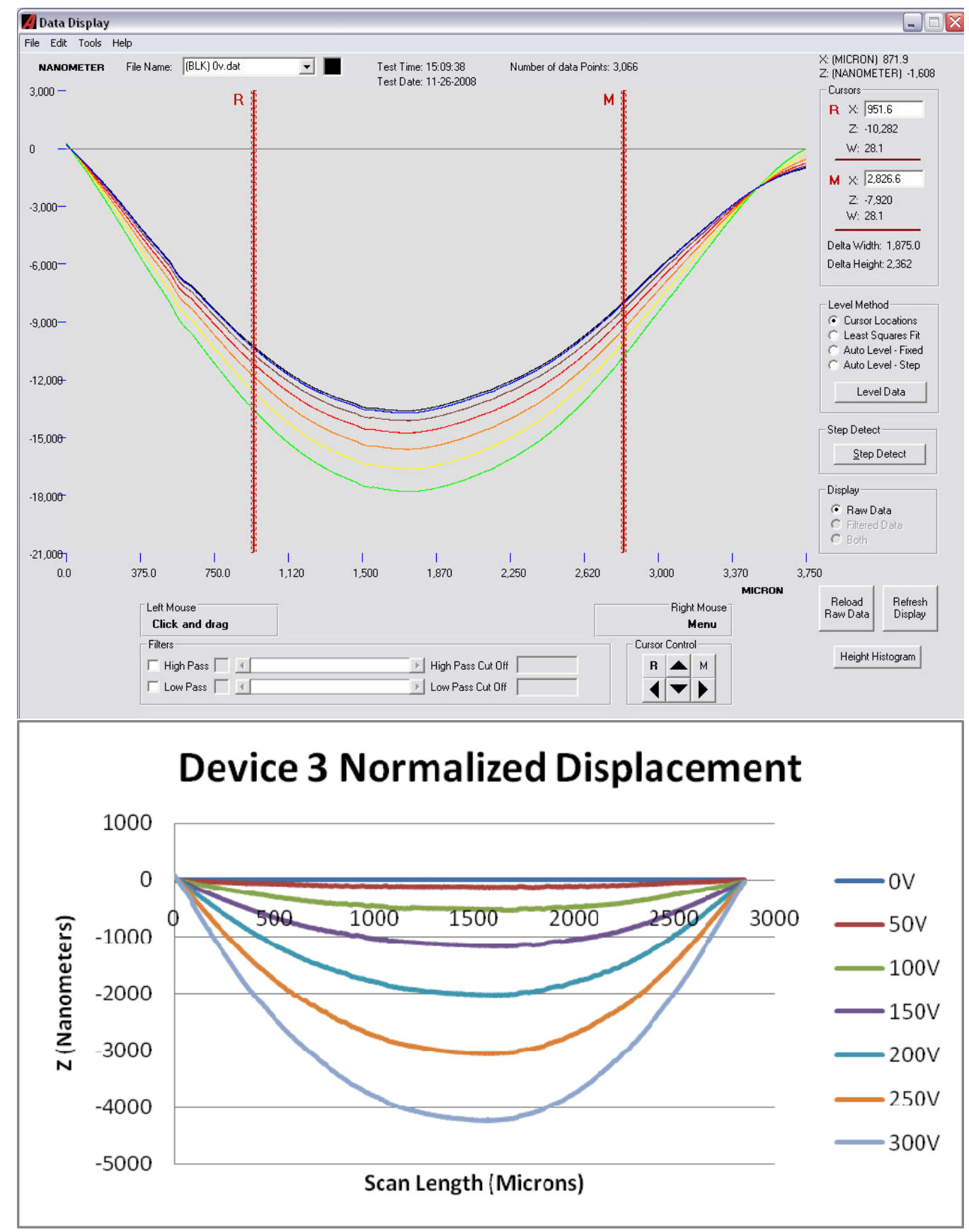

Raw and Normalized Deflection Data for Device 3, Test 3 


\section{APPENDIX B Cont'd}

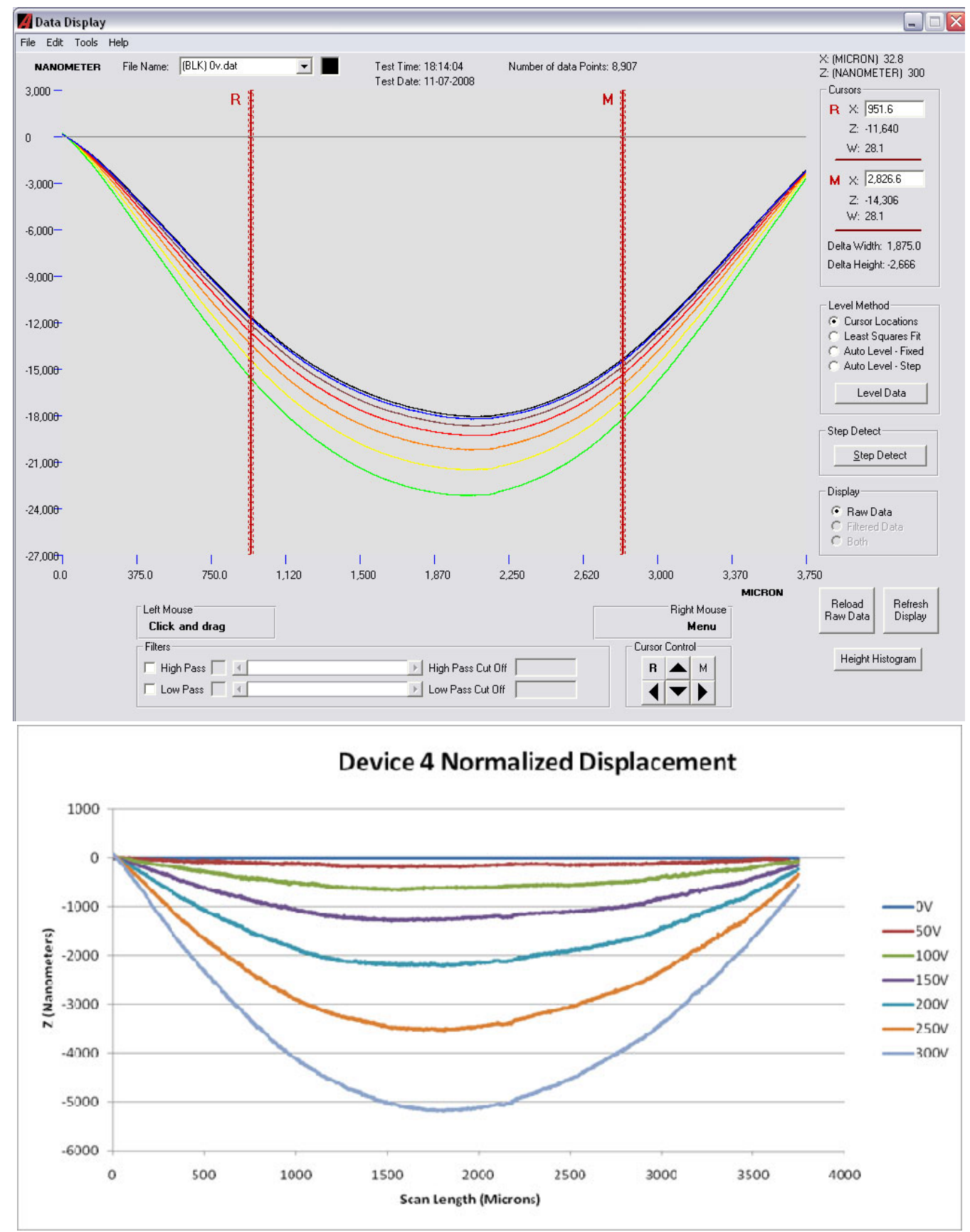

Raw and Normalized Deflection Data for Device 4, Test 1 


\section{APPENDIX B Cont'd}

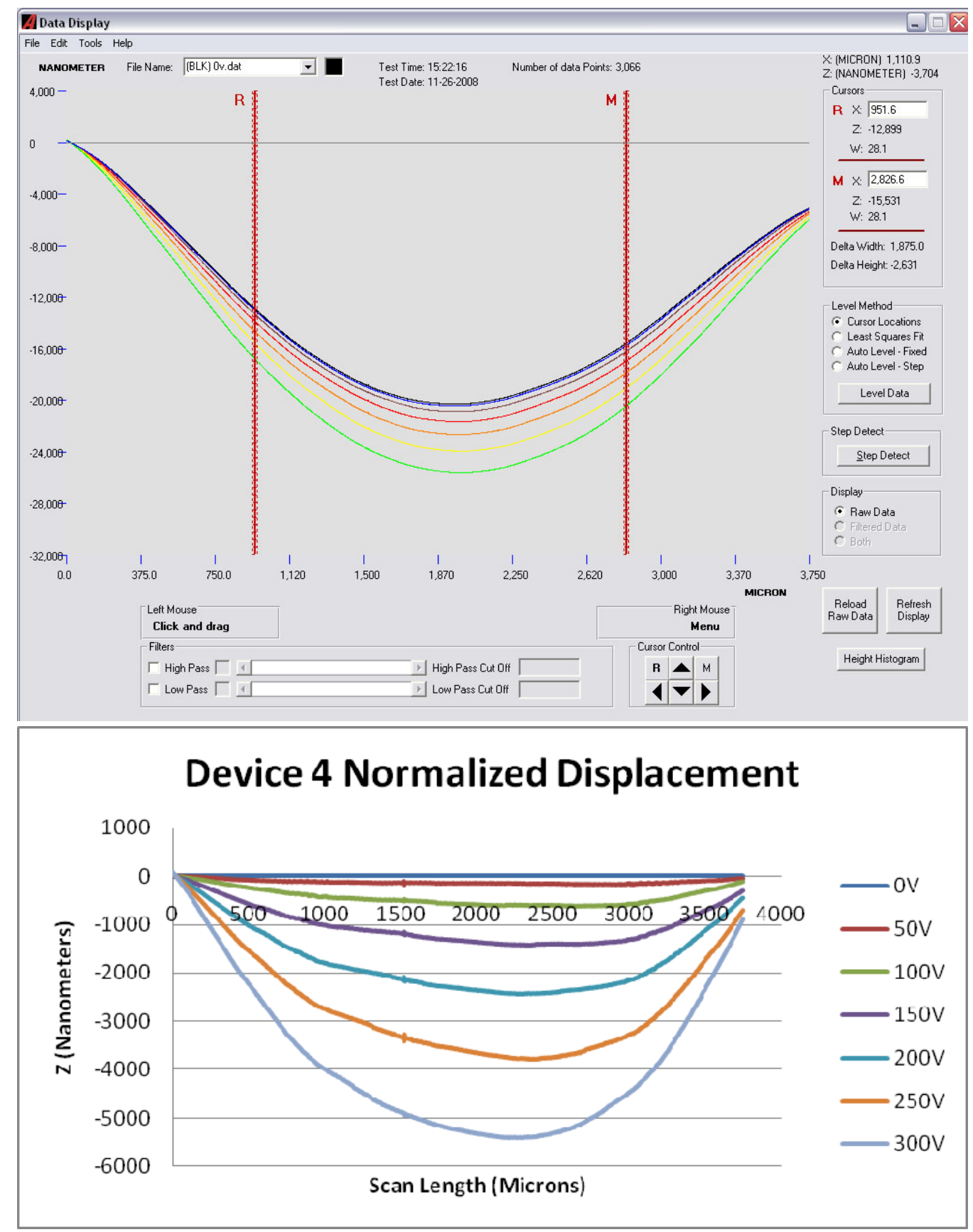

Raw and Normalized Deflection Data for Device 4, Test 2 


\section{APPENDIX B Cont'd}

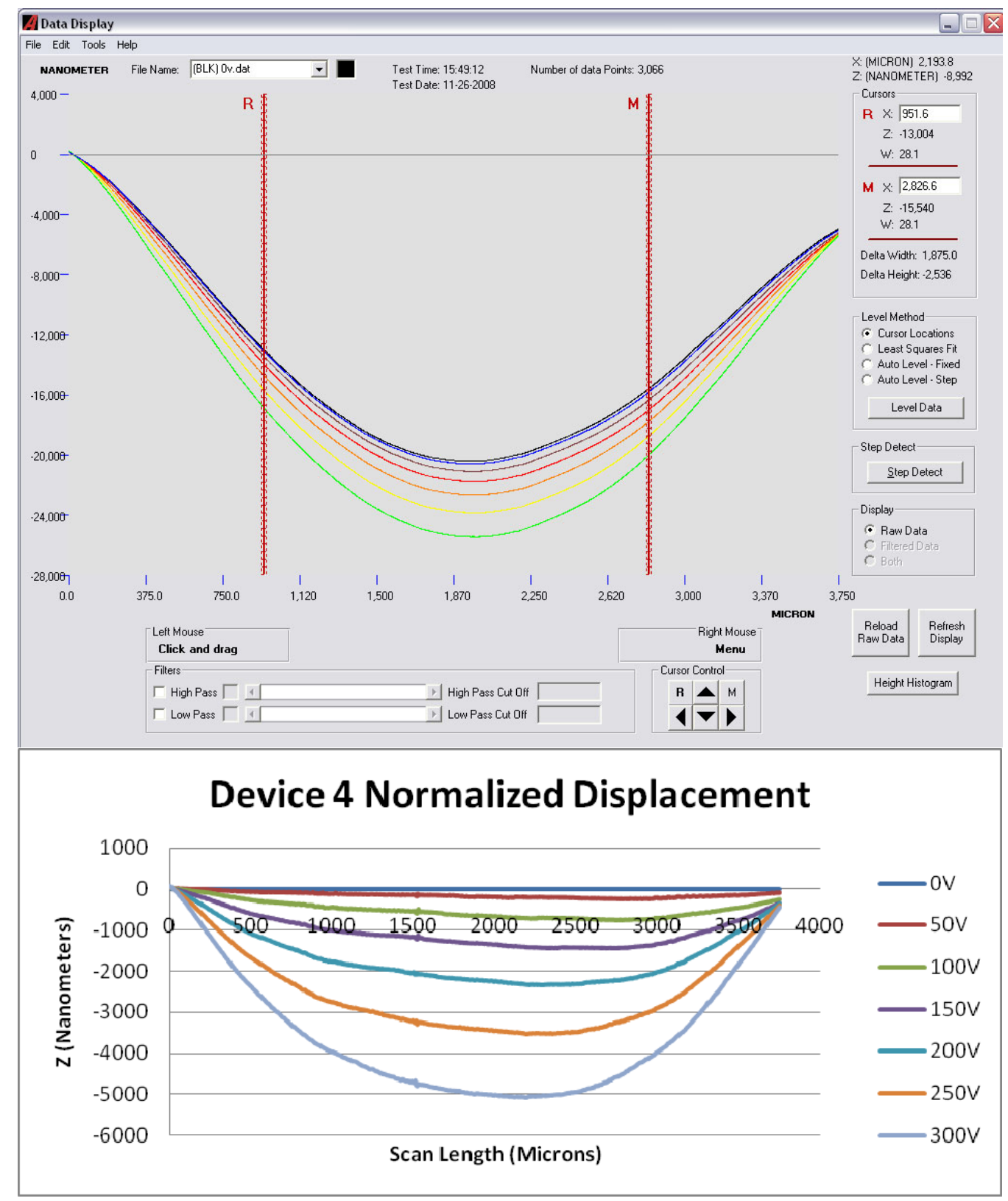

Raw and Normalized Deflection Data for Device 4, Test 3 


\section{APPENDIX B Cont'd}

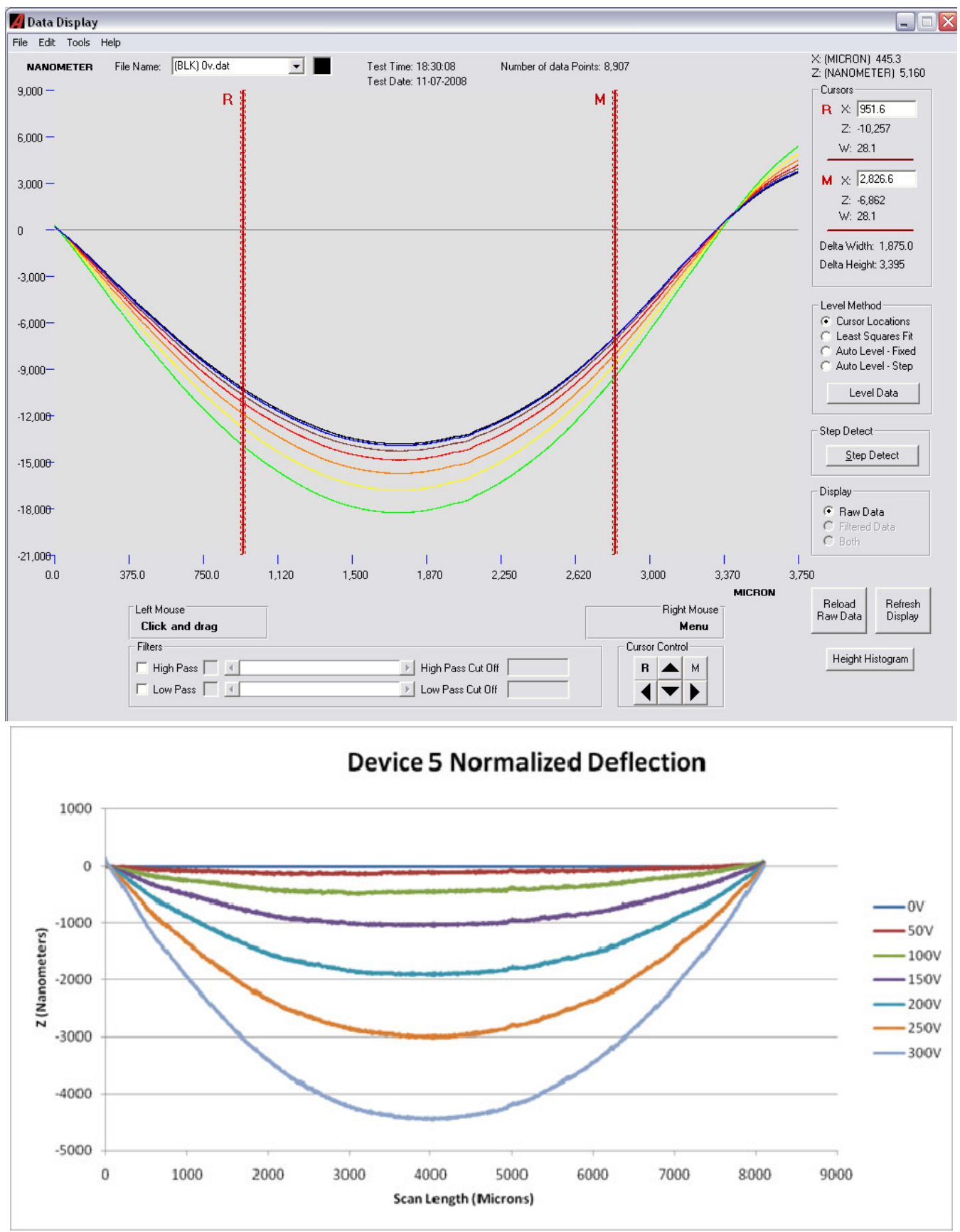

Raw and Normalized Deflection Data for Device 5 


\section{APPENDIX B Cont'd}

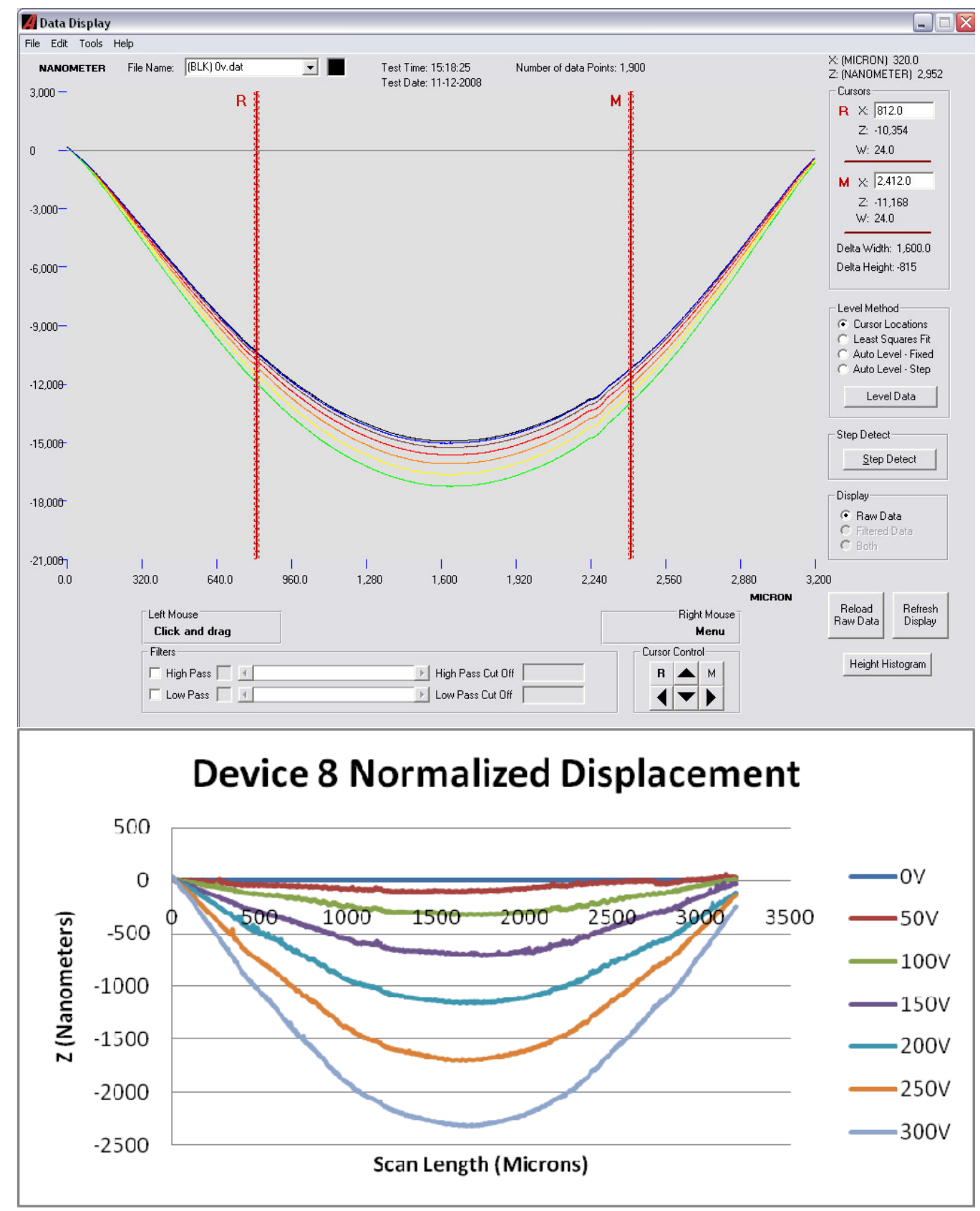

Raw and Normalized Deflection Data for Device 8 


\section{APPENDIX B Cont'd}

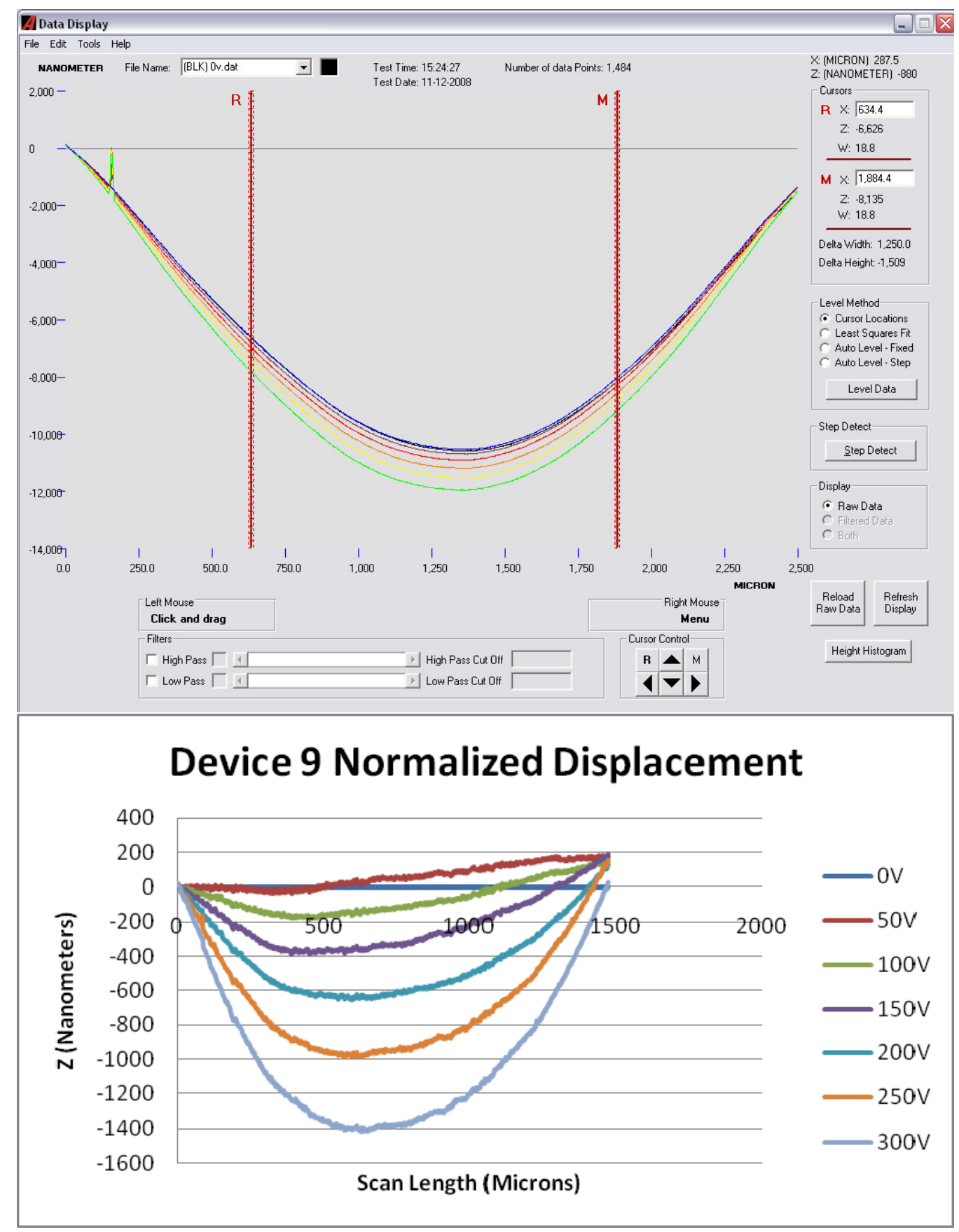

Raw and Normalized Deflection Data for Device 9 


\section{APPENDIX B Cont'd}

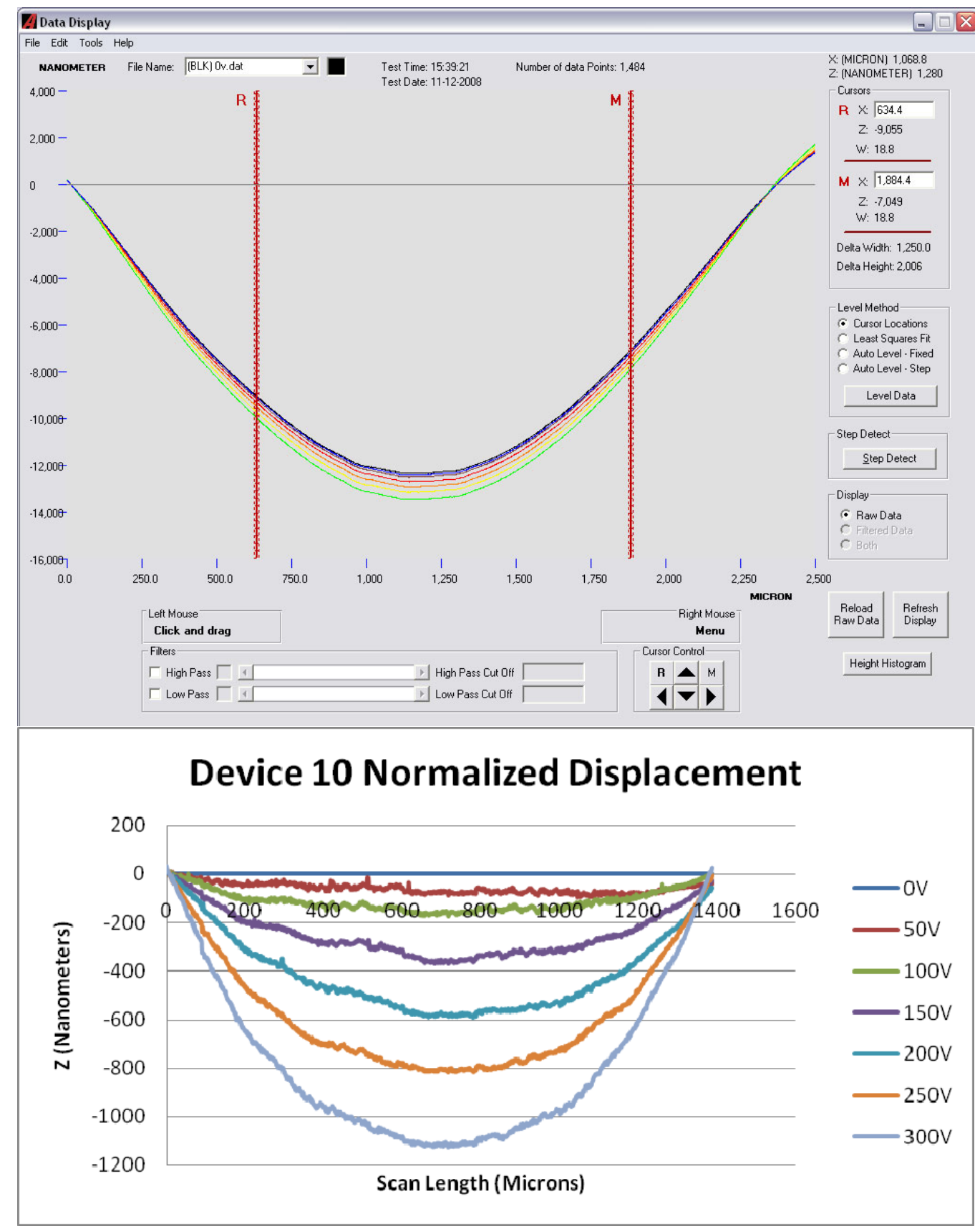

Raw and Normalized Deflection Data for Device 10 


\section{APPENDIX C: Maximum Deflection}

\begin{tabular}{|ccccc|}
\hline \multicolumn{5}{c|}{ Device 1 } \\
Voltage & \multicolumn{4}{c|}{ Max. Deflection (nm) } \\
$(\mathrm{V})$ & Test 1 & Test 2 & Test 3 & Average \\
0 & 0 & 0 & 0 & 0 \\
50 & 62 & 10 & 42 & 38 \\
100 & 267 & 119 & 183 & 189.6667 \\
150 & 562 & 361 & 382 & 435 \\
200 & 975 & 622 & 710 & 769 \\
250 & 1542 & 1046 & 1098 & 1228.667 \\
300 & 2265 & 1565 & 1670 & 1833.333 \\
\hline
\end{tabular}

\begin{tabular}{|ccccr|}
\hline \multicolumn{5}{c|}{ Device 2 } \\
Voltage & \multicolumn{4}{c|}{ Max. Deflection (nm) } \\
(V) & Test 1 & Test 2 & Test 3 & \multicolumn{1}{c|}{ Average } \\
0 & 0 & 0 & 0 & 0 \\
50 & 164 & 184 & 218 & 188.6667 \\
100 & 662 & 736 & 691 & 696.3333 \\
150 & 1508 & 1607 & 1568 & 1561 \\
200 & 2773 & 2779 & 2777 & 2776.333 \\
250 & 4289 & 4317 & 4354 & 4320 \\
300 & 5992 & 6067 & 6067 & 6042 \\
\hline
\end{tabular}

\begin{tabular}{|ccccr|}
\hline \multicolumn{5}{c|}{ Device 3 } \\
Voltage & \multicolumn{4}{c|}{ Max. Deflection (nm) } \\
(V) & Test 1 & Test 2 & Test 3 & Average \\
0 & 0 & 0 & 0 & 0 \\
50 & 250 & 267 & 130 & 215.6667 \\
100 & 844 & 735 & 545 & 708 \\
150 & 1769 & 1506 & 1179 & 1484.667 \\
200 & 2851 & 2521 & 2036 & 2469.333 \\
250 & 4292 & 3079 & 3056 & 3475.667 \\
300 & 5930 & 5051 & 4234 & 5071.667 \\
\hline
\end{tabular}




\section{APPENDIX C Cont'd}

\begin{tabular}{|ccccr|}
\hline \multicolumn{5}{c|}{ Device 4 } \\
Voltage & \multicolumn{4}{c|}{ Max. Deflection (nm) } \\
(V) & Test 1 & Test 2 & Test 3 & \multicolumn{1}{c|}{ Average } \\
0 & 0 & 0 & 0 & 0 \\
50 & 203 & 178 & 234 & 205 \\
100 & 670 & 637 & 770 & 692.3333 \\
150 & 1315 & 1431 & 1430 & 1392 \\
200 & 2238 & 2452 & 2334 & 2341.333 \\
250 & 3552 & 3782 & 3526 & 3620 \\
300 & 5205 & 5415 & 5084 & 5234.667 \\
\hline
\end{tabular}

\begin{tabular}{|ccccc|}
\hline $\begin{array}{c}\text { Voltag } \\
\text { e (V) }\end{array}$ & $\begin{array}{c}\text { Device 5 } \\
(4.4 \mathrm{~mm})\end{array}$ & $\begin{array}{c}\text { Device 8 } \\
(3.7 \mathrm{~mm})\end{array}$ & $\begin{array}{c}\text { Device } 9 \\
(3.0 \mathrm{~mm})\end{array}$ & $\begin{array}{c}\text { Device 10 } \\
(3.0 \mathrm{~mm})\end{array}$ \\
0 & $\begin{array}{c}\text { Max. Deflection } \\
(\mathrm{nm})\end{array}$ & $\begin{array}{c}\text { Deflection } \\
(\mathrm{nm})\end{array}$ & $\begin{array}{c}\text { Max. Deflection } \\
(\mathrm{nm})\end{array}$ & $\begin{array}{c}\text { Max. Deflection } \\
(\mathrm{nm})\end{array}$ \\
50 & 0 & 0 & 0 & 0 \\
100 & 165 & 116 & 35 & 97 \\
150 & 507 & 333 & 185 & 179 \\
200 & 1069 & 718 & 389 & 371 \\
250 & 1943 & 1161 & 656 & 591 \\
300 & 3028 & 1708 & 986 & 819 \\
\hline
\end{tabular}




\section{APPENDIX D: Deflection-Voltage Curves}
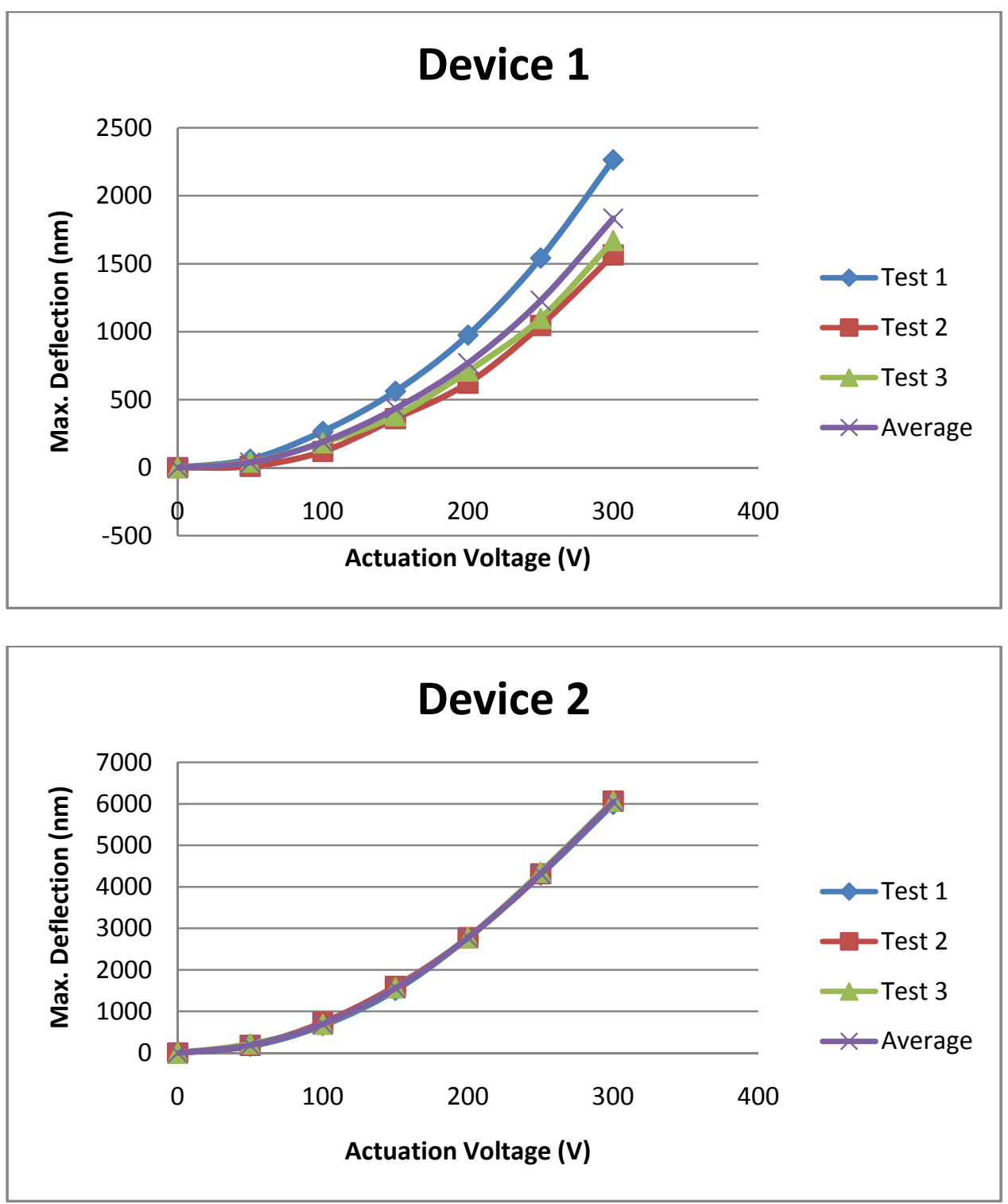


\section{APPENDIX D Cont'd}
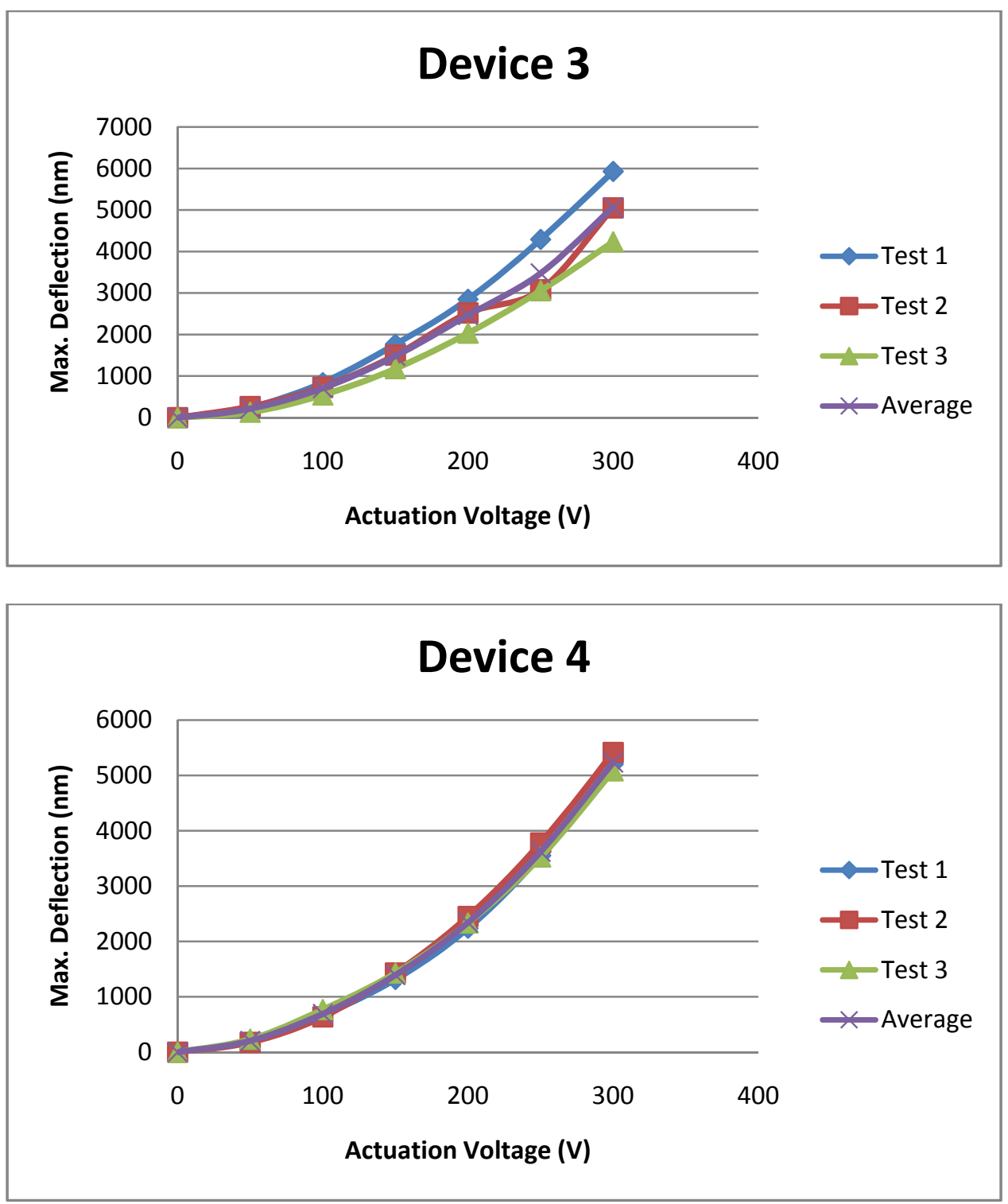


\section{APPENDIX D Cont'd}
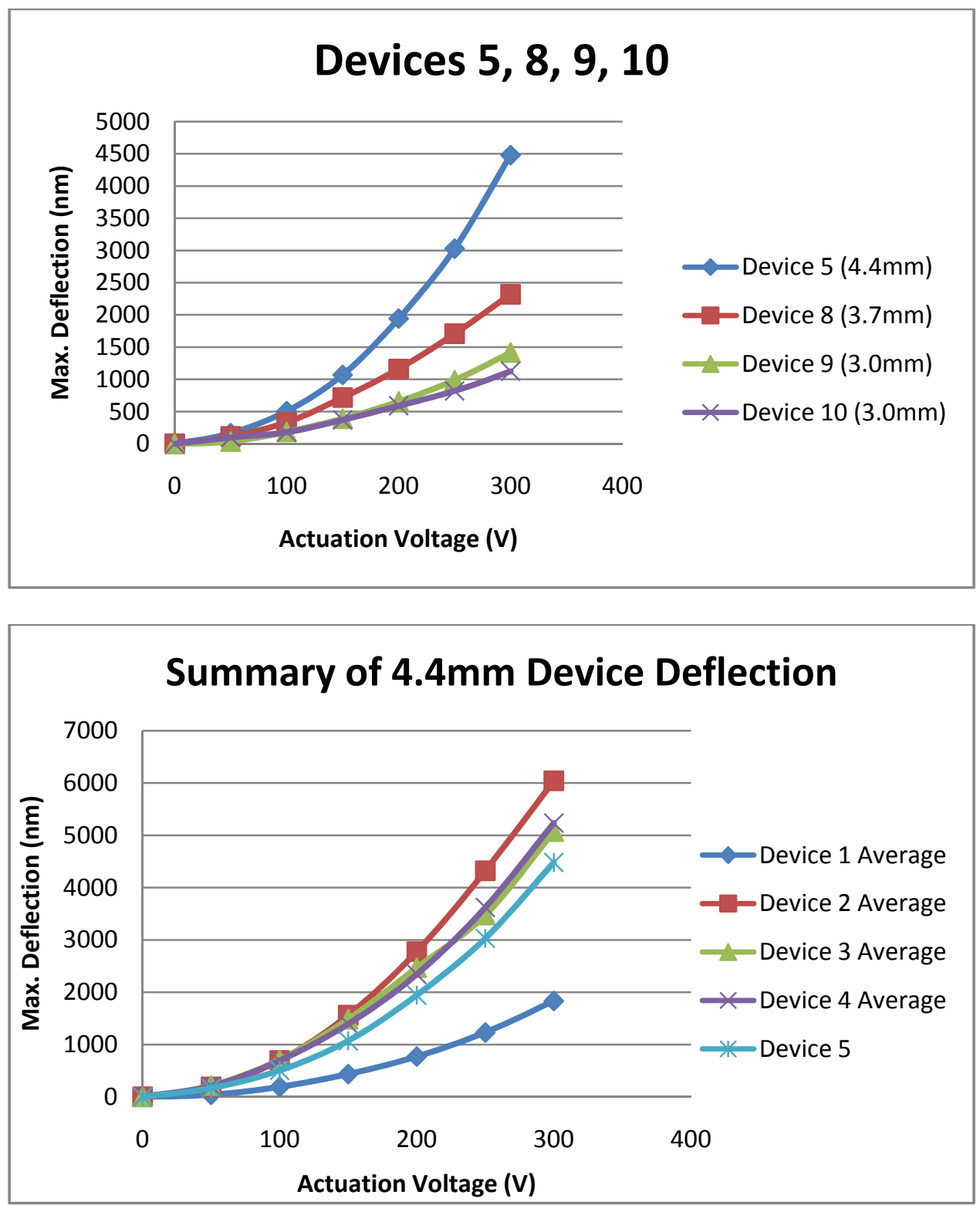


\section{APPENDIX E: Initial Design Equation Model Comparison}

\begin{tabular}{|c|c|c|c|c|c|c|}
\hline Device \# & Voltage (V) & Measured $(\mu \mathrm{m})$ & $\begin{array}{l}\text { Original } \\
\text { Prediction } \\
(\mu \mathrm{m})\end{array}$ & Fitting Factor & $\begin{array}{l}\text { Scaled } \\
\text { Prediction } \\
(\mu \mathrm{m})\end{array}$ & Difference \\
\hline \multirow{8}{*}{1} & 0 & 0 & 0 & & 0 & \\
\hline & 50 & 0.038 & 0.547437842 & 14.40625899 & 0.04655315 & 0.225082897 \\
\hline & 100 & 0.189666667 & 2.189751367 & 11.54526204 & 0.1862126 & 0.018211246 \\
\hline & 150 & 0.435 & 4.926940575 & 11.32630017 & 0.418978351 & 0.036831378 \\
\hline & 200 & 0.769 & 8.759005467 & 11.39012414 & 0.744850401 & 0.0314039 \\
\hline & 250 & 1.2286666667 & 13.68594604 & 11.13886005 & 1.163828752 & 0.052770956 \\
\hline & 300 & 1.833333333 & 19.7077623 & 10.74968853 & 1.675913403 & 0.085865417 \\
\hline & & & Average: & 11.75941565 & & \\
\hline \multirow{8}{*}{2} & 0 & 0 & 0 & & 0 & \\
\hline & 50 & 0.1886666667 & 0.547437842 & 2.901614002 & 0.174832361 & 0.073326708 \\
\hline & 100 & 0.696333333 & 2.189751367 & 3.144688416 & 0.699329444 & 0.004302696 \\
\hline & 150 & 1.561 & 4.926940575 & 3.156271989 & 1.57349125 & 0.008002082 \\
\hline & 200 & 2.776333333 & 8.759005467 & 3.154882507 & 2.797317777 & 0.00755833 \\
\hline & 250 & 4.32 & 13.68594604 & 3.168043065 & 4.370809027 & 0.011761349 \\
\hline & 300 & 6.042 & 19.7077623 & 3.261794489 & 6.2939649999 & 0.041702251 \\
\hline & & & Average: & 3.131215745 & & \\
\hline \multirow{8}{*}{3} & 0 & 0 & 0 & & 0 & \\
\hline & 50 & 0.2156666667 & 0.547437842 & 2.538351662 & 0.161641963 & 0.250500944 \\
\hline & 100 & 0.708 & 2.189751367 & 3.092869162 & 0.646567852 & 0.08676857 \\
\hline & 150 & 1.484666667 & 4.926940575 & 3.318550006 & 1.454777668 & 0.020131791 \\
\hline & 200 & 2.469333333 & 8.759005467 & 3.547113445 & 2.586271409 & 0.047356132 \\
\hline & 250 & 3.4756666667 & 13.68594604 & 3.937646315 & 4.041049077 & 0.162668767 \\
\hline & 300 & 5.071666667 & 19.7077623 & 3.885855202 & 5.819110671 & 0.147376406 \\
\hline & & & Average: & 3.386730965 & & \\
\hline \multirow{8}{*}{4} & 0 & 0 & 0 & & 0 & \\
\hline & 50 & 0.205 & 0.547437842 & 2.670428496 & 0.15899031 & 0.224437511 \\
\hline & 100 & 0.692333333 & 2.189751367 & 3.162857054 & 0.635961241 & 0.08142334 \\
\hline & 150 & 1.392 & 4.926940575 & 3.539468804 & 1.430912793 & 0.027954592 \\
\hline & 200 & 2.341333333 & 8.759005467 & 3.741033087 & 2.543844965 & 0.086494148 \\
\hline & 250 & 3.62 & 13.68594604 & 3.780648078 & 3.974757757 & 0.09799938 \\
\hline & 300 & 5.234666667 & 19.7077623 & 3.764855254 & 5.72365117 & 0.09341273 \\
\hline & & & Average: & 3.443215129 & & \\
\hline
\end{tabular}




\section{APPENDIX E Cont'd}

\begin{tabular}{|c|c|c|c|c|c|c|}
\hline Device \# & Voltage (V) & Measured $(\mu \mathrm{m})$ & $\begin{array}{l}\text { Original } \\
\text { Prediction } \\
(\mu \mathrm{m})\end{array}$ & Fitting Factor & $\begin{array}{l}\text { Scaled } \\
\text { Prediction } \\
(\mu \mathrm{m})\end{array}$ & Difference \\
\hline \multirow{8}{*}{5} & 0 & 0 & 0 & & 0 & \\
\hline & 50 & 0.165 & 0.547437842 & 3.317805101 & 0.127942918 & 0.224588375 \\
\hline & 100 & 0.507 & 2.189751367 & 4.319036226 & 0.511771672 & 0.009411583 \\
\hline & 150 & 1.069 & 4.926940575 & 4.608924766 & 1.151486263 & 0.077162079 \\
\hline & 200 & 1.943 & 8.759005467 & 4.507980168 & 2.047086689 & 0.053570092 \\
\hline & 250 & 3.028 & 13.68594604 & 4.51979724 & 3.198572952 & 0.056331886 \\
\hline & 300 & 4.48 & 19.7077623 & 4.399054085 & 4.605945051 & 0.028112735 \\
\hline & & & Average: & 4.278766265 & & \\
\hline \multirow{8}{*}{8} & 0 & 0 & 0 & & 0 & \\
\hline & 50 & 0.116 & 0.273735425 & 2.35978815 & 0.063975316 & 0.448488652 \\
\hline & 100 & 0.333 & 1.094941701 & 3.288113217 & 0.255901265 & 0.231527732 \\
\hline & 150 & 0.718 & 2.463618828 & 3.431223995 & 0.575777847 & 0.198080993 \\
\hline & 200 & 1.161 & 4.379766806 & 3.772408963 & 1.023605062 & 0.118341894 \\
\hline & 250 & 1.708 & 6.843385634 & 4.006666062 & 1.599382909 & 0.063593145 \\
\hline & 300 & 2.319 & 9.854475313 & 4.249450329 & 2.303111389 & 0.006851493 \\
\hline & & & Average: & 3.517941786 & & \\
\hline \multirow{8}{*}{9} & 0 & 0 & 0 & & 0 & \\
\hline & 50 & 0.035 & 0.118306642 & 3.380189772 & 0.027649709 & 0.210008315 \\
\hline & 100 & 0.185 & 0.473226568 & 2.557981449 & 0.110598836 & 0.402168454 \\
\hline & 150 & 0.389 & 1.064759778 & 2.737171667 & 0.248847381 & 0.360289509 \\
\hline & 200 & 0.656 & 1.892906273 & 2.885527854 & 0.442395344 & 0.325616854 \\
\hline & 250 & 0.986 & 2.957666051 & 2.999661309 & 0.691242725 & 0.29894247 \\
\hline & 300 & 1.416 & 4.259039113 & 3.007795984 & 0.995389523 & 0.297041297 \\
\hline & & & Average: & 2.928054673 & & \\
\hline \multirow{8}{*}{10} & 0 & 0 & 0 & & 0 & \\
\hline & 50 & 0.097 & 0.118306642 & 1.219656103 & 0.027649709 & 0.714951454 \\
\hline & 100 & 0.179 & 0.473226568 & 2.643723844 & 0.110598836 & 0.382129408 \\
\hline & 150 & 0.371 & 1.064759778 & 2.869972448 & 0.248847381 & 0.329252343 \\
\hline & 200 & 0.591 & 1.892906273 & 3.202887094 & 0.442395344 & 0.251446119 \\
\hline & 250 & 0.819 & 2.957666051 & 3.611313859 & 0.691242725 & 0.155991789 \\
\hline & 300 & 1.125 & 4.259039113 & 3.785812545 & 0.995389523 & 0.115209312 \\
\hline & & & Average: & 2.888894316 & & \\
\hline
\end{tabular}




\section{APPENDIX E Cont'd}
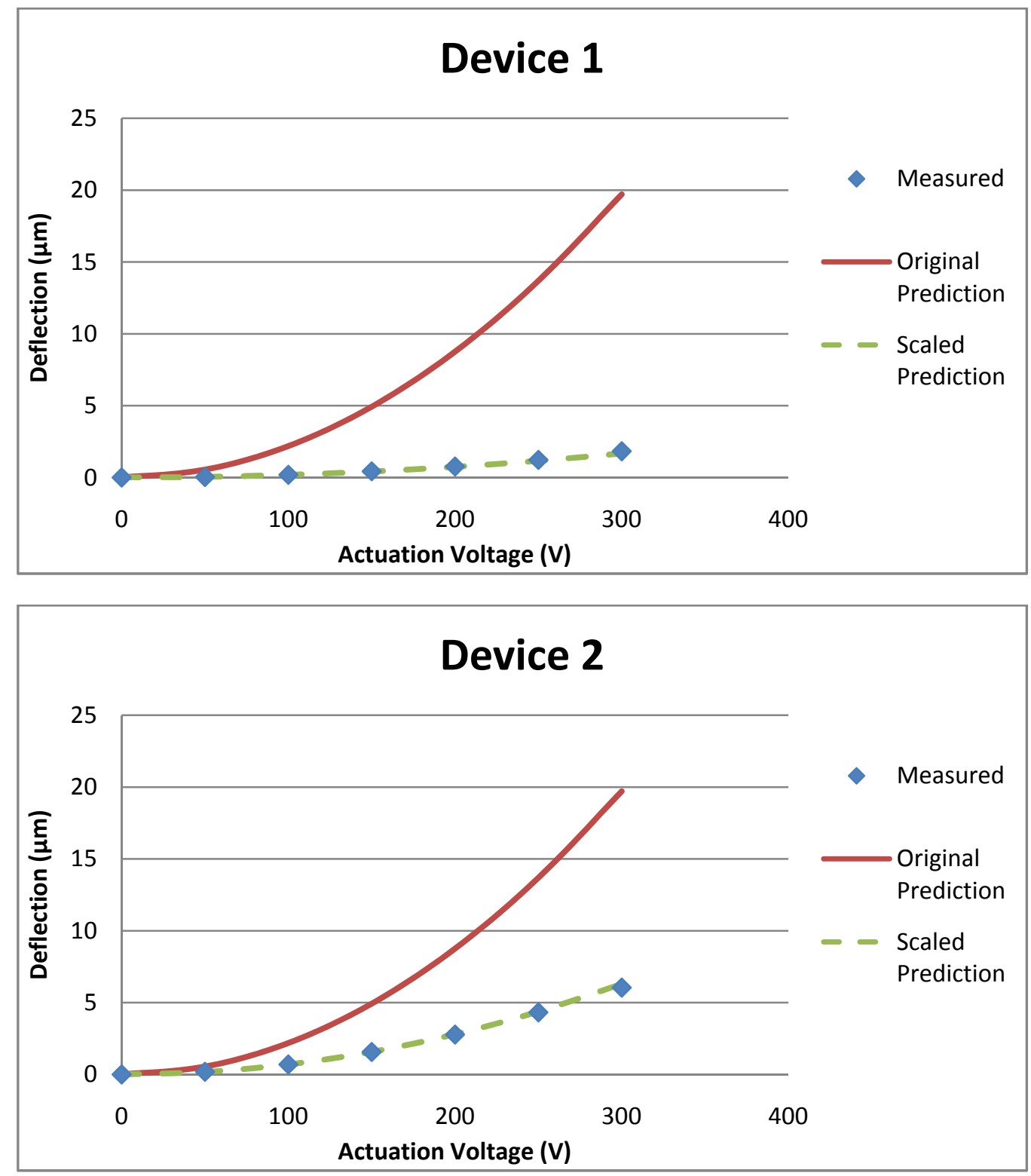


\section{APPENDIX E Cont'd}
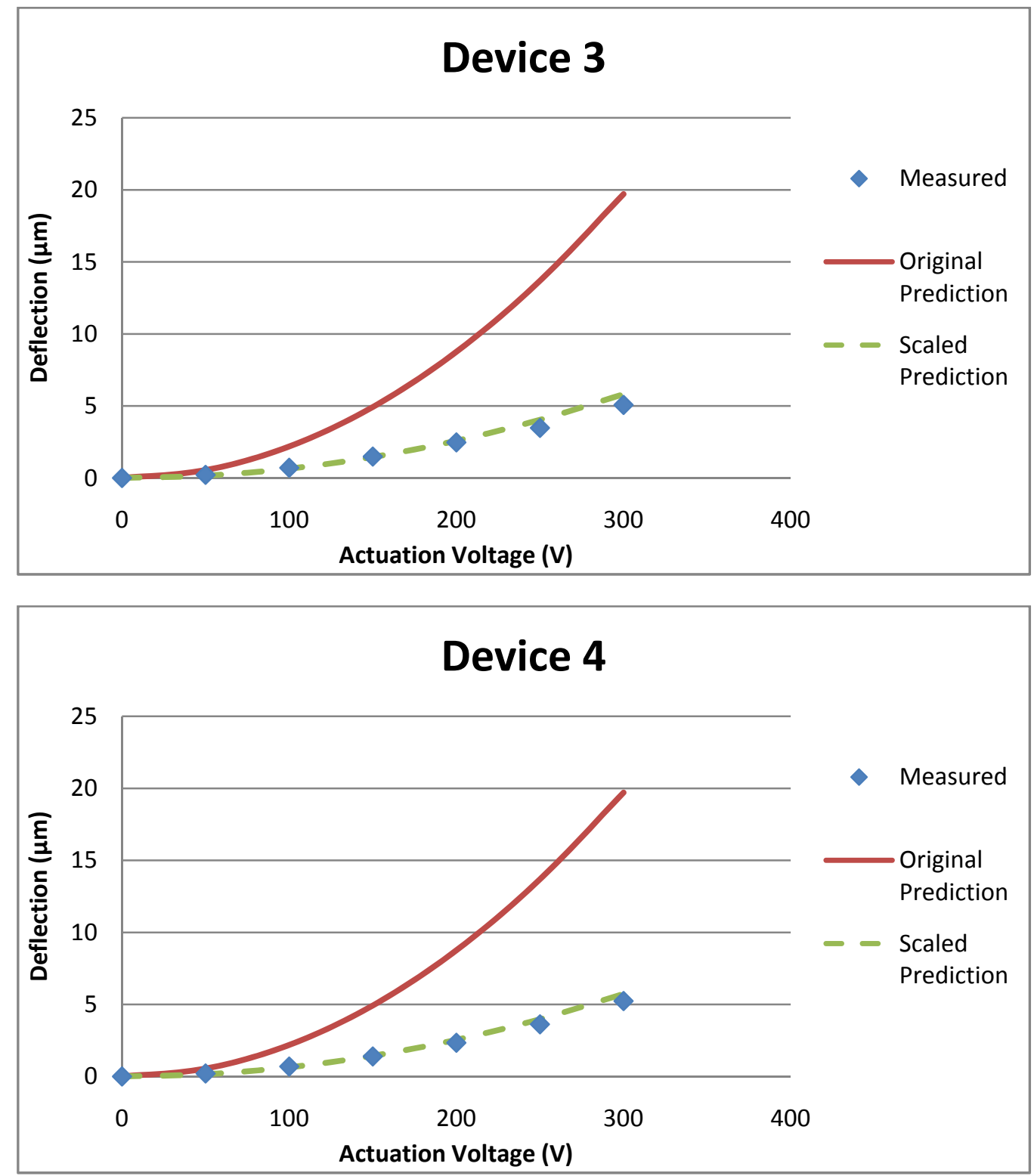


\section{APPENDIX E Cont'd}
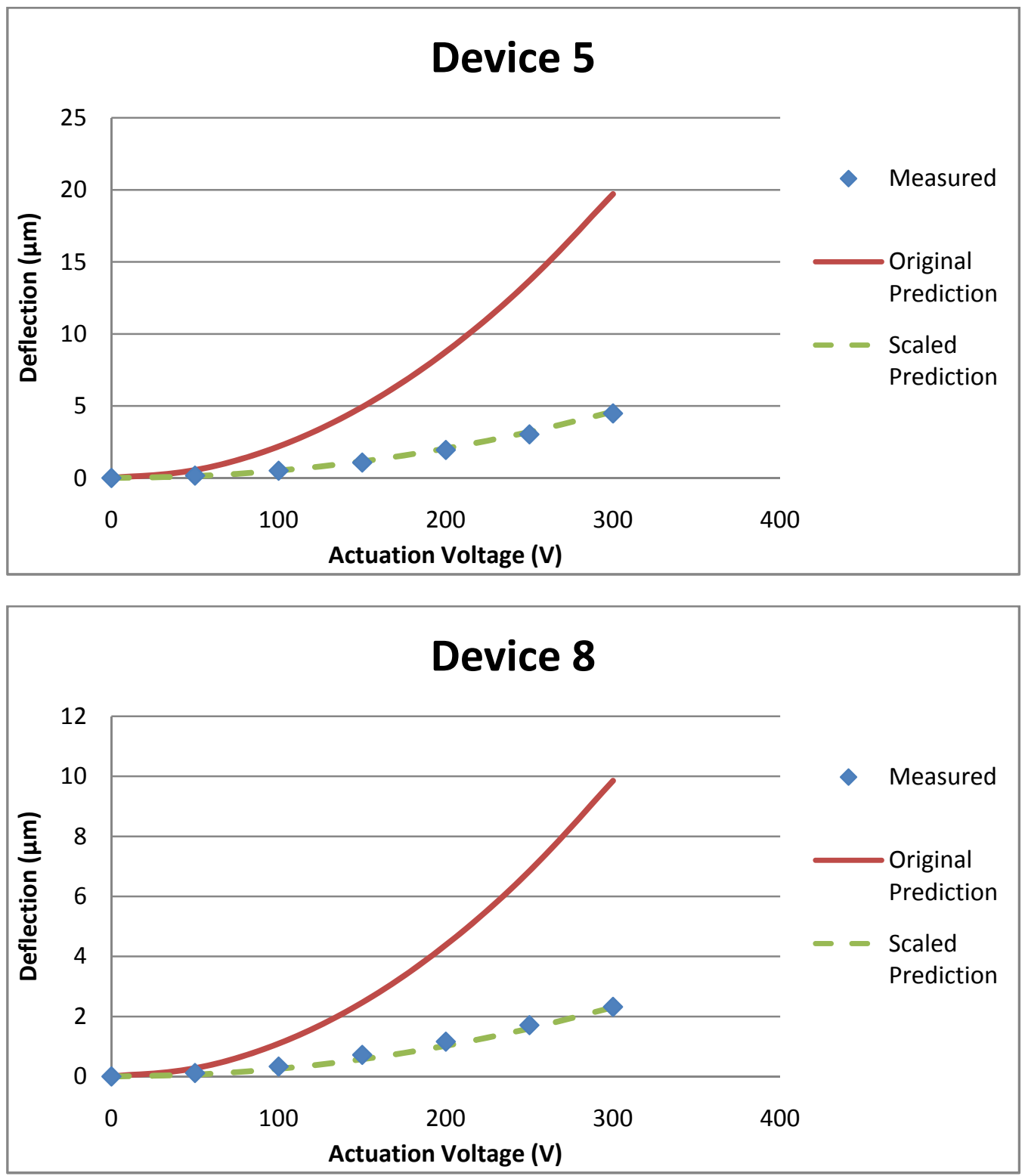


\section{APPENDIX E Cont'd}
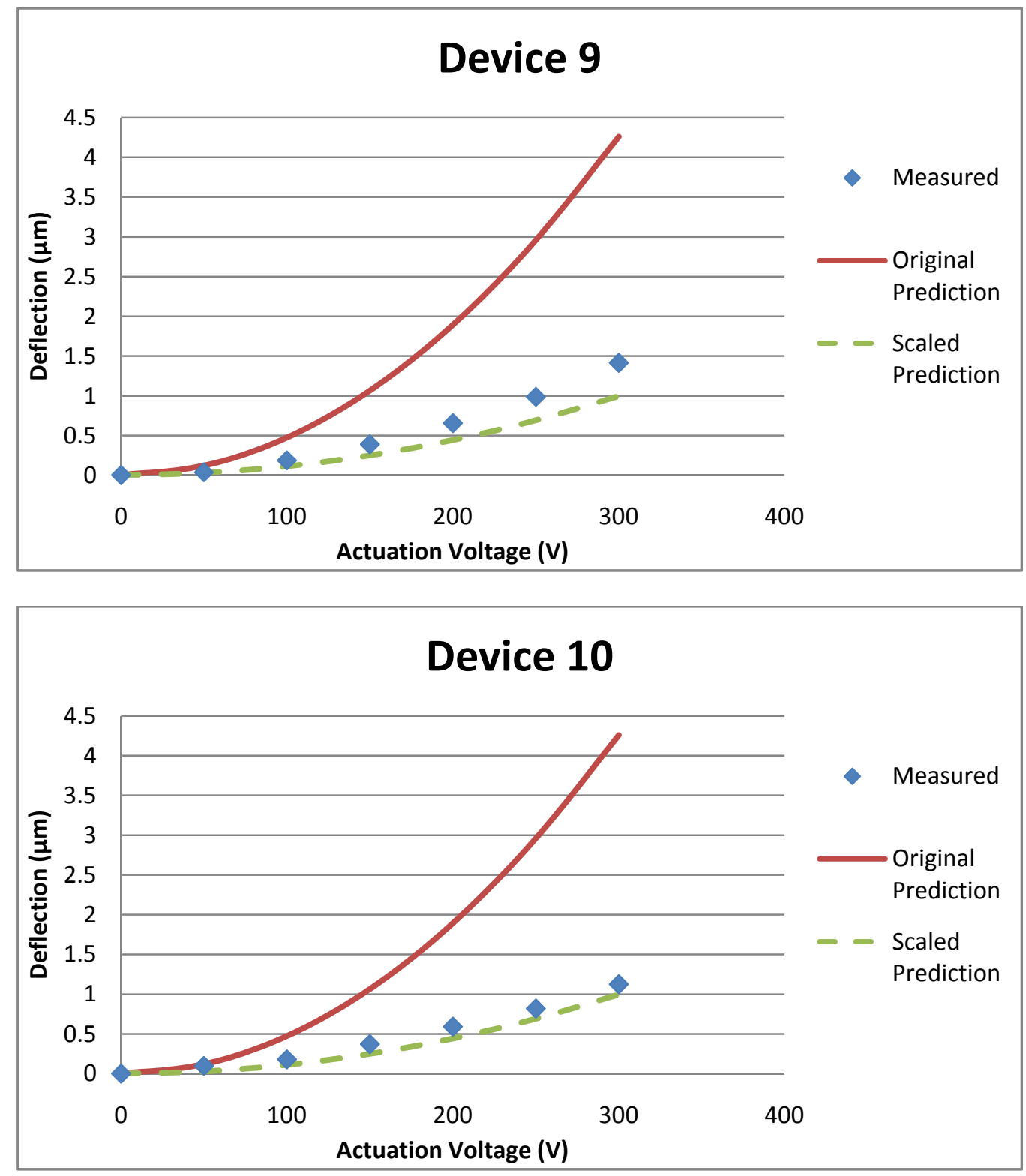